\title{
2000 Annual Interim Sanitary Landfill Groundwater Monitoring Report
}

by



J. A. Chase

Westinghouse Savannah River Company

Savannah River Site

Aiken, South Carolina 29808

This paper was prepared in connection with work done under the above contract number with the U. S. Department of Energy. By acceptance of this paper, the publisher and/or recipient acknowledges the U. S. Government's right to retain a nonexclusive, royalty-free license in and to any copyright covering this paper, along with the right to reproduce and to authorize others to reproduce all or part of the copyrighted paper. 


\title{
INTERIM SANITARY LANDFILL GROUNDWATER MONITORING REPORT (U)
}

\author{
2000 ANNUAL
}

Publication Date: JANUARY 2001

Westinghouse Savannah River Company Savannah River Site

Aiken, SC 29808 


\section{DISCLAIMER}

This report was prepared as an account of work sponsored by an agency of the United States Government. Neither the United States Government nor any agency thereof, nor any of their employees, makes any warranty, express or implied, or assumes any legal liability or responsibility for the accuracy, completeness, or usefulness of any information, apparatus, product or process disclosed, or represents that its use would not infringe privately owned rights. Reference herein to any specific commercial product, process or service by trade name, trademark, manufacturer, or otherwise does not necessarily constitute or imply its endorsement, recommendation, or favoring by the United States Government or any agency thereof. The views and opinions of authors expressed herein do not necessarily state or reflect those of the United States Government or any agency thereof.

This report has been reproduced directly from the best available copy.

Available for sale to the public, in paper, from: U.S. Department of Commerce, National Technical Information Service, 5285 Port Royal Road, Springfield, VA 22161

phone: (800) 553-6847

fax: (703) 605-6900

email: orders@ntis.fedworld.gov

online ordering: http://www.ntis.gov/ordering.htm

Available electronically at http://www.doe.gov/bridge

Available for a processing fee to U.S. Department of Energy and its contractors, in paper, from: U.S. Department of Energy, Office of Scientific and Technical Information, P.O. Box 62, Oak Ridge, TN 37831-0062 phone: (865) 576-8401

fax: (865) 576-5728

email: reports@adonis.osti.gov 


\title{
CERTIFICATION
}

\author{
INTERIM SANITARY LANDFILL \\ 2000 ANNUAL GROUNDWATER \\ MONITORING REPORT (U) \\ WSRC-TR-2000-000464
}

[REF: R.61-79.270.11(d)]

"I certify under penalty of law that this document and all attachments were prepared under my direction or supervision in accordance with a system designed to assure that qualified personnel properly gather and evaluate the information submitted. Based on my inquiry of the person or persons who manage the system, or those persons directly responsible for gathering the information, the information submitted is, to the best of my knowledge and belief, true, accurate, and complete. I am aware that there are significant penalties for submitting false information, including the possibility of fine and imprisonment of knowing violations."

Signature on File

WSRC as Co-Operator

Date Signed

M. A. Sabbe

Vice President \& General Manager

Environmental Restoration Division

Signature on File

D. T. Bignell

Date Signed

Manager

Environmental Protection Department 
THIS PAGE INTENTIONALLY LEFT BLANK. 


\section{Contents}

Description $\quad \underline{\text { Page }}$

Introduction....................................................................... 1

Discussion......................................................................... 2

Flow Direction and Rate.................................................... 2

Analytical Result................................................................. 3

Conclusion....................................................................... 5

\section{APPENDICES}

Appendix A -.- Groundwater Protection Standards........................... A - 1

Appendix B -- Groundwater Monitoring Results Tables ....................... B - 1

Appendix C -. Figures......................................................... C - 1

Appendix D -- Vadose Methane Monitoring Data................................ D - 1 
THIS PAGE INTENTIONALLY LEFT BLANK. 
The SRS Interim Sanitary Landfill (ISL) opened in mid-1992 and operated until October 1999 under Domestic Waste Permit \# 025500-1102. The ISL was subsequently closed and officially began the 30 year post-closure care period as of September 9, 2000, which is the effective date of the modified permit. This annual monitoring report was prepared in accordance with the modified permit and the SCDHEC approved Closure/Post-Closure Plan, Q-CLP-G-00008, Rev. 2, October 26, 1998.

Fifteen wells shown in Figure 1 were each sampled semi-annually during Calendar Year 2000 in accordance with Closure/Post-Closure Plan, Q-CLP-G-00008, Rev. 2, October 26, 1998. Sampling was performed during the first and third quarters of 2000 . Sampling and analysis for the Appendix II list was performed in first quarter, and again in the third quarter for any analytes that were detected above background. Sampling and analysis for the Appendix I list was performed during third quarter 2000. The list of analytes is presented in Appendix A, Groundwater Protection Standards. The analytical results appear in Appendix B for the First and Second Halves of 2000 . The vadose zone methane monitoring data is presented and discussed in Appendix D.

The well sampling and analyses were conducted in accordance with WSRC Procedure Manual 3Q5, Hydrogeologic Data Collection.

The report includes a discussion of the groundwater flow direction and rate, the groundwater analytical results, and the methane monitoring results. A key to reading the data tables in the preface of Appendix B. Figures are provided in Appendix C. 


\section{DISCUSSION}

\section{Flow Direction and Rate}

The uppermost aquifer beneath the ISL is referred to as the Steed Pond Aquifer. All previous studies indicate that flow in the Steed Pond was to the southeast. Potentiometric data from the wells in the vicinity of the ISL are generally consistent with the regional flow. Figures 2,3 and 4 show the potentiometric surface for three quarters spanning over a year. The flow direction is consistent over this period, showing a southerly and east-southeasterly component (relative to true north) that may be related to increased recharge over the ISL. The increased recharge would result from the previous condition of the waste cell as a low excavated area that would collect runoff. The closure of the ISL and associated cover placement over the waste cell may influence this observed flow pattern over time.

Flow rate can be estimated using the following equation:

$$
\text { Flow }(\mathrm{ft} / \text { day })=\frac{\text { Hydraulic Conductivity }(\mathrm{ft} / \mathrm{day})}{\text { Porosity (unitless) }} \times \frac{\mathrm{dh}(\mathrm{ft})}{\mathrm{dl}(\mathrm{ft})}
$$

where the hydraulic conductivity is assumed to be $16 \mathrm{ft} /$ day, the effective porosity value is 20 percent, the change in head is $\mathrm{dh}$, and the horizontal distance along each flow arrow is $\mathrm{dl}$.

The flow rate was estimated in two directions indicated by flow arrows A-A' and B-B' on Figures 3 and 4.

Based on first quarter 2000 potentiometric data (Figure 3), the calculations are as follows:

Line A-A'

$(16 / .20) \times(4 \mathrm{ft} / 261.4 .0 \mathrm{ft})=1.22 \mathrm{ft} /$ day or $446.85 \mathrm{ft} /$ year

Line B-B'

$16 / .20 \times 4 \mathrm{ft} / 603.5 \mathrm{ft}=0.53 \mathrm{ft} /$ day or $193.5 \mathrm{ft} /$ year

Based on third quarter 2000 potentiometric data (Figure 4), the calculations are as follows:

Line A-A'

$16 / .20 \times 4 \mathrm{ft} / 273.6 \mathrm{ft}=1.16 \mathrm{ft} /$ day or $426.9 \mathrm{ft} /$ year

Line B-B'

$16 / .20 \times 4 \mathrm{ft} / 668.7 \mathrm{ft}=0.48 \mathrm{ft} /$ day or $174.7 \mathrm{ft} /$ year 


\section{Analytical Results}

\section{Groundwater Monitoring}

The analytical data for the first and third quarter groundwater sampling events are provided in Appendix B. Results for analytes that exceeded the Groundwater Protection Standards (GWPS) shown in Table A.1 are discussed in the following text.

During first quarter 2000, five wells had analytes detected at levels exceeding GWPSs.

- Mercury (total recoverable) was elevated in well LFW-34 with a maximum concentration of $3.49 \mathrm{ug} / \mathrm{L}$. However, mercury was not detected in the next sampling event (third quarter 2000).

- Trichlorofluoromethane was elevated in three wells; LFW-34 with a maximum concentration of $490 \mathrm{ug} / \mathrm{L}$, LFW 32 with a concentration of $160 \mathrm{ug} / \mathrm{L}$, and LFW 74D with a concentration of $21 \mathrm{ug} / \mathrm{L}$.

- Zinc was elevated in two wells above the GWPS tolerance limit of $29.3 \mathrm{ug} / \mathrm{L}$ Appendix I constituents; LFW 76 with a maximum concentration of $35.6 \mathrm{ug} / \mathrm{L}$, and LFW 78 with a maximum concentration of $32.7 \mathrm{ug} / \mathrm{L}$.

During third quarter 2000, five wells had analytes detected at levels exceeding GWPSs.

a Copper (total recoverable) was elevated above the GWPS tolerance limit of $29.8 \mathrm{ug} / \mathrm{L}$, Appendix I constituents in well LFW 31, with a maximum concentration of $40.8 \mathrm{ug} / \mathrm{L}$. However, this concentration is less than the Primary Drinking Water Standard of 1000 ug/L.

- Dichloromethane (methylene chloride) was elevated in well LFW 43B, with a maximum concentration of $8.32 \mathrm{ug} / \mathrm{L}$. This is a background well.

- Specific Conductance was elevated above the GWPS tolerance limits for Appendix I constituents in well LFW 74D, with a value of $48.5 \mathrm{uS} / \mathrm{cm}$.

口 Thallium (total recoverable) was elevated in well LFW 43D, with a maximum concentration of $10.1 \mathrm{ug} / \mathrm{L}$. This is a background well.

- Trichlorofluoromethane was elevated in well LFW 34, with a maximum concentration of $450 \mathrm{ug} / \mathrm{L}$.

- Zinc was elevated above the GWPS tolerance limit of $29.3 \mathrm{ug} / \mathrm{L}$, Appendix I in well LFW 31, with a maximum concentration of $40.2 \mathrm{ug} / \mathrm{L}$ 


\section{Methane Monitoring}

Results of the methane monitoring are provided in Appendix D, Table D.1. Methane was sampled at methane monitoring wells LGM 1,2,3 and 4. The results ranged from 0.51 vol\% to 53.7 vol\%. Methane levels exceeded the 5\% LEL at LGM 1 and LGM 4.

In addition, extensive multilevel soil gas sampling was done around the Interim Sanitary Landfill to assess the extent to which methane in the soil had migrated outward from the landfill. The results were submitted to SCDHEC on September $28^{\text {th }}, 2000$ (letter, D.G. Wells to R.E.

Schweitzer). The results of the soil gas sampling show that little or no migration has occurred. The submittal to SCDHEC also proposed fitting the existing methane monitoring wells with check valves. 


\section{CONCLUSION}

Potentiometric data from the wells in the vicinity of the ISL are generally consistent with the southeast regional flow. Figures 2, 3, and 4 indicate a flow direction with southerly and eastsoutheasterly components (relative to true north), that may be related to increased recharge over the ISL. The closure of the ISL and associated cover placement over the waste cell may influence this observed flow pattern over time. Ground water flow rates range from 175 to 447 $\mathrm{ft} /$ year.

Mercury was detected above its GWPS of $2.0 \mathrm{ug} / \mathrm{L}$ (Final PDWS) in well LFW-34 downgradient of the ISL. Mercury is being monitored semiannually along with Appendix I analytes. Trichlorofluoromethane was detected above its GWPS of $20 \mathrm{ug} / \mathrm{L}$ (EPA Method 8240 Detection Limit) in three wells in the first quarter 2000 and one well (LFW 34) in the third quarter 2000. Well LFW 44D, which is about 500 feet downgradient from LFW 34, will be added to the assessment monitoring program. Assessment monitoring will continue for Zinc, Copper, Thallium, and Dichloromethane.

No new analytes were identified that were detected above the GWPS.

The vadose zone methane monitoring data and prior correspondence to the SCDHEC discussing the data is included in Appendix D. 
THIS PAGE INTENTIONALLY LEFT BLANK. 


\section{Appendix A}

\section{Groundwater Protection Standards}


Table A.1 Groundwater Protection Standards

\begin{tabular}{|c|c|}
\hline$\underline{\mathrm{CAS}}$ & Analyte Name \\
\hline 630206 & 1,1,1,2-Tetrachloroethane \\
\hline 71556 & 1,1,1-Trichloroethane \\
\hline 79345 & 1,1,2,2-Tetrachloroethane \\
\hline 79005 & 1,1,2-Trichloroethane \\
\hline 75343 & 1,1-Dichloroethane \\
\hline 75354 & 1,1-Dichloroethene \\
\hline 563586 & 1,1-Dichloropropene \\
\hline 96184 & 1,2,3-Trichloropropane \\
\hline 95943 & 1,2,4,5-Tetrachlorobenzene \\
\hline 120821 & 1,2,4-Trichlorobenzene \\
\hline 96128 & 1,2-Dibromo-3-chloropropane \\
\hline 106934 & 1,2-Dibromoethane \\
\hline 95501 & 1,2-Dichlorobenzene \\
\hline 107062 & 1,2-Dichloroethane \\
\hline 78875 & 1,2-Dichloropropane \\
\hline 99354 & 1,3,5-Trinitrobenzene \\
\hline 541731 & 1,3-Dichlorobenzene \\
\hline 142289 & 1,3-Dichloropropane \\
\hline 99650 & 1,3-Dinitrobenzene \\
\hline 106467 & 1,4-Dichlorobenzene \\
\hline 130154 & 1,4-Naphthoquinone \\
\hline 134327 & 1-Naphthylamine \\
\hline 594207 & 2,2-Dichloropropane \\
\hline 108601 & 2,2-Oxybis(1-chloropropane) \\
\hline 58902 & 2,3,4,6-Tetrachlorophenol \\
\hline 93765 & $2,4,5-\mathrm{T}$ \\
\hline 95954 & 2,4,5-Trichlorophenol \\
\hline 88062 & 2,4,6-Trichlorophenol \\
\hline 120832 & 2,4-Dichlorophenol \\
\hline 94757 & 2,4-Dichlorophenoxyacetic acid \\
\hline 105679 & 2,4-Dimethyl phenol \\
\hline 51285 & 2,4-Dinitrophenol \\
\hline 121142 & 2,4-Dinitrotoluene \\
\hline 87650 & 2,6-Dichlorophenol \\
\hline 606202 & 2,6-Dinitrotoluene \\
\hline 53963 & 2-Acetylaminofluorene \\
\hline 91587 & 2-Chloronaphthalene \\
\hline 95578 & 2-Chlorophenol \\
\hline 591786 & 2-Hexanone \\
\hline 534521 & 2-Methyl-4,6-dinitrophenol \\
\hline 91576 & 2-Methylnaphthalene \\
\hline 91598 & 2-Naphthylamine \\
\hline 88744 & 2-Nitroaniline \\
\hline 88755 & 2-Nitrophenol \\
\hline 88857 & 2-sec-Butyl-4,6-dinitrophenol \\
\hline 91941 & 3,3'-Dichlorobenzidine \\
\hline 119937 & 3,3'-Dimethoxybenzidine \\
\hline 56495 & 3-Methylcholanthrene \\
\hline
\end{tabular}

Synonym

o-Dichlorobenzene

sym-Trinitrobenzene

m-Dichlorobenzene

m-Dinitrobenzene

p-Dichlorobenzene

Picloram

2,4,5-Trichlorophenoxyacetic acid

2,4-D

4,6-Dintro-o-cresol

o-Nitroaniline

o-Nitrophenol

Dinoseb

3,3'-Dimethylbenzidine $\underline{\text { Unit LimitSource }} \dagger$

$\mu \mathrm{g} / \mathrm{L} \quad 20$ EPA Method 8240

$\mu \mathrm{g} / \mathrm{L} \quad 200$ Final PDWS (EPA, 1998a)

$\mu \mathrm{g} / \mathrm{L} \quad 100$ EPA Method 8240

$\mu \mathrm{g} / \mathrm{L} \quad 5 \quad$ Final PDWS (EPA, 1998a)

$\mu \mathrm{g} / \mathrm{L} \quad 20$ EPA Method 8240

$\mu \mathrm{g} / \mathrm{L} \quad 7 \quad$ Final PDWS (EPA, 1998a)

ug/L 5 EPA Method 8260B

$\mu \mathrm{g} / \mathrm{L} \quad 20 \quad$ EPA Method 8240

$\mu \mathrm{g} / \mathrm{L} \quad 162$ EPA Method 8270

$\mu \mathrm{g} / \mathrm{L} \quad 70 \quad$ Final PDWS (EPA, 1998a)

$\mu \mathrm{g} / \mathrm{L} \quad 0.2$ Final PDWS (EPA, 1998a)

$\mu \mathrm{g} / \mathrm{L} \quad 0.05$ Final PDWS (EPA, 1998a)

$\mu g / L \quad 600$ Final PDWS (EPA, 1998a)

$\mu \mathrm{g} / \mathrm{L} \quad 5 \quad$ Final PDWS (EPA, 1998a)

$\mu \mathrm{g} / \mathrm{L} \quad 5 \quad$ Final PDWS (EPA, 1998a)

$\mu \mathrm{g} / \mathrm{L} \quad 162$ EPA Method 8270

$\mu \mathrm{g} / \mathrm{L} \quad 162$ EPA Method 8270

ug/L 5 EPAMethod $8260 \mathrm{~B}$

$\mu \mathrm{g} / \mathrm{L} \quad 162$ EPA Method 8270

$\mu \mathrm{g} / \mathrm{L} \quad 75$ Final PDWS (EPA, 1998a)

$\mu \mathrm{g} / \mathrm{L} \quad 162$ EPA Method 8270

$\mu \mathrm{g} / \mathrm{L} \quad 162$ EPA Method 8270

$\mu \mathrm{g} / \mathrm{L} \quad 10 \quad$ EPA Method 8260

$\mu \mathrm{g} / \mathrm{L} \quad 500$ Final PDWS (EPA, 1998a)

$\mu \mathrm{g} / \mathrm{L} \quad 167$ EPA Method 8270

$\mu \mathrm{g} / \mathrm{L} \quad 0.5 \quad$ EPA Method 8150

$\mu \mathrm{g} / \mathrm{L} \quad 10 \quad$ EPA Method 8270

$\mu \mathrm{g} / \mathrm{L} \quad 1 \quad$ EPA Method 8270

$\mu \mathrm{g} / \mathrm{L} \quad 10.2$ EPA Method 8270

$\mu \mathrm{g} / \mathrm{L} \quad 70 \quad$ Final PDWS (EPA, 1998a)

$\mu \mathrm{g} / \mathrm{L} \quad 10.2$ EPA Method 8270

$\mu \mathrm{g} / \mathrm{L} \quad 102$ EPA Method 8270

$\mu \mathrm{g} / \mathrm{L} \quad 1 \quad$ EPA Method 8270

$\mu \mathrm{g} / \mathrm{L} \quad 167 \quad$ EPA Method 8270

$\mu \mathrm{g} / \mathrm{L} \quad 1 \quad$ EPA Method 8270

$\mu \mathrm{g} / \mathrm{L} \quad 162$ EPA Method 8270

$\mu \mathrm{g} / \mathrm{L} \quad 10.2$ EPA Method 8240

$\mu \mathrm{g} / \mathrm{L} \quad 10.2$ EPA Method 8270

$\mu \mathrm{g} / \mathrm{L} \quad 100$ EPA Method 8240

$\mu \mathrm{g} / \mathrm{L} \quad 102 \quad$ EPA Method 8270

$\mu \mathrm{g} / \mathrm{L} \quad 10 \quad$ EPA Method 8270

$\mu \mathrm{g} / \mathrm{L} \quad 162$ EPA Method 8270

$\mu \mathrm{g} / \mathrm{L} \quad 10 \quad$ EPA Method 8270

ug/L $\quad 10.2$ EPA Method 8270

ug/L 7 Final PDWS (EPA, 1998a)

$\mu \mathrm{g} / \mathrm{L} \quad 10.2$ EPA Method 8270

$\mu \mathrm{g} / \mathrm{L} \quad 162$ EPA Method 8270

$\mu \mathrm{g} / \mathrm{L} \quad 162$ EPA Method 8270 


\begin{tabular}{|c|c|c|}
\hline$\underline{\mathbf{C A S}}$ & Analyte Name & Synonym \\
\hline$\overline{92671}$ & $\overline{\text { 4-Aminobiphenyl }}$ & a- Aminobiphenyl \\
\hline 101553 & 4-Bromophenyl phenyl ether & \\
\hline 106478 & 4-Chloroaniline & p-Chloroaniline \\
\hline 59507 & 4-Chloro-m-cresol & p-Cholo-m-cresol \\
\hline 7005723 & 4-Chlorophenyl phenyl ether & \\
\hline 100016 & 4-Nitroaniline & p-Nitroaniline \\
\hline 100027 & 4-Nitrophenol & p-Nitrophenol \\
\hline 99558 & 5-Nitro-o-toluidine & \\
\hline 57976 & 7,12-Dimethylbenz[a]anthracene & \\
\hline 83329 & Acenaphthene & \\
\hline 208968 & Acenaphthylene & \\
\hline 67641 & Acetone & \\
\hline 75058 & Acetonitrile (Methyl cyanide) & \\
\hline 98862 & Acetophenone & \\
\hline 107028 & Acrolein & \\
\hline 107131 & Acrylonitrile & \\
\hline 309002 & Aldrin & \\
\hline 107051 & Allyl chloride & \\
\hline 319846 & alpha-Benzene hexachloride & alpha-BHC \\
\hline 120127 & Anthracene & \\
\hline 7440360 & Antimony, total recoverable & \\
\hline 7440382 & Arsenic, total recoverable & \\
\hline 7440393 & Barium, total recoverable & \\
\hline 71432 & Benzene & \\
\hline 56553 & Benzo[a]anthracene & \\
\hline 50328 & Benzo[a]pyrene & \\
\hline 205992 & Benzo[b]fluoranthene & \\
\hline 191242 & Benzo[g,h,i]perylene & \\
\hline 207089 & Benzo[k]fluoranthene & \\
\hline 100516 & Benzyl alcohol & \\
\hline 7440417 & Beryllium, total recoverable & \\
\hline 319857 & beta-Benzene hexachloride & beta-BHC \\
\hline 111911 & Bis(2-chloroethoxy) methane & bis[2-chloro-1-methylethyl] ether \\
\hline 111444 & Bis(2-chloroethyl) ether & \\
\hline 117817 & Bis(2-ethylhexyl) phthalate & \\
\hline 74975 & Bromochloromethane & \\
\hline 75274 & Bromodichloromethane & \\
\hline 75252 & Bromoform & \\
\hline 74839 & Bromomethane & Methyl bromide \\
\hline 85687 & Butylbenzyl phthalate & \\
\hline 7440439 & Cadmium, total recoverable & \\
\hline 75150 & Carbon disulfide & \\
\hline 56235 & Carbon tetrachloride & \\
\hline 57749 & Chlordane & \\
\hline 108907 & Chlorobenzene & \\
\hline 510156 & Chlorobenzilate & \\
\hline 75003 & Chloroethane & \\
\hline 75014 & Chloroethene (Vinyl chloride) & Vinyl chloride \\
\hline 67663 & Chloroform & \\
\hline 74873 & Chloromethane & (Methyl chloride) \\
\hline
\end{tabular}

$\underline{\text { Unit }} \underline{\text { Limit Source }} \dagger$

$\mu \mathrm{g} / \mathrm{L} \quad 162$ EPA Method 8270

$\mu \mathrm{g} / \mathrm{L} \quad 10.2$ EPA Method 8270

$\mu \mathrm{g} / \mathrm{L} \quad 10 \quad$ EPA Method 8270

$\mu \mathrm{g} / \mathrm{L} \quad 10.2$ EPA Method 8270

$\mu \mathrm{g} / \mathrm{L} \quad 10.2$ EPA Method 8270

$\mu \mathrm{g} / \mathrm{L} \quad 10 \quad$ EPA Method 8270

$\mu \mathrm{g} / \mathrm{L} \quad 10.2$ EPA Method 8270

$\mu \mathrm{g} / \mathrm{L} \quad 162$ EPA Method 8270

$\mu \mathrm{g} / \mathrm{L} \quad 162$ EPA Method 8270

$\mu \mathrm{g} / \mathrm{L} \quad 10.2$ EPA Method 8270

$\mu \mathrm{g} / \mathrm{L} \quad 10.2$ EPA Method 8270

$\mu \mathrm{g} / \mathrm{L} \quad 20$ EPA Method 8260B

$\mu \mathrm{g} / \mathrm{L} \quad 100$ EPA Method 8240

$\mu \mathrm{g} / \mathrm{L} \quad 170$ EPA Method 8270

$\mu \mathrm{g} / \mathrm{L} \quad 333$ EPA Method 8240

$\mu \mathrm{g} / \mathrm{L} \quad 500$ EPA Method 8240

$\mu \mathrm{g} / \mathrm{L} \quad 0.8 \quad$ EPA Method 8080

$\mu \mathrm{g} / \mathrm{L} \quad 833$ EPA Method 8240

$\mu \mathrm{g} / \mathrm{L} \quad 0.3 \quad$ EPA Method 8080

$\mu \mathrm{g} / \mathrm{L} \quad 10.2$ EPA Method 8270

$\mu \mathrm{g} / \mathrm{L} 6$ Final PDWS (EPA, 1998a)

$\mu g / L \quad 50$ Final PDWS (EPA, 1998a)

$\mu \mathrm{g} / \mathrm{L} \quad 2000$ Final PDWS (EPA, 1998a)

$\mu \mathrm{g} / \mathrm{L} 55$ Final PDWS (EPA, 1998a)

$\mu \mathrm{g} / \mathrm{L} \quad 0.1 \quad$ Proposed PDWS (EPA, 1990)

$\mu \mathrm{g} / \mathrm{L} \quad 0.2$ Final PDWS (EPA, 1998a)

$\mu \mathrm{g} / \mathrm{L} \quad 0.2$ Proposed PDWS (EPA, 1990)

$\mu \mathrm{g} / \mathrm{L} \quad 10.2$ EPA Method 8270

$\mu \mathrm{g} / \mathrm{L} \quad 0.2$ Proposed PDWS (EPA, 1990)

$\mu \mathrm{g} / \mathrm{L} \quad 10 \quad$ EPA Method 8270

$\mu \mathrm{g} / \mathrm{L} 4 \quad$ Final PDWS (EPA, 1998a)

$\mu \mathrm{g} / \mathrm{L} \quad 0.5 \quad$ EPA Method 8080

$\mu \mathrm{g} / \mathrm{L} \quad 10.2$ EPA Method 8270

$\mu \mathrm{g} / \mathrm{L} \quad 10.2$ EPA Method 8270

$\mu \mathrm{g} / \mathrm{L} \quad 6 \quad$ Final PDWS (EPA, 1998a)

$\mu \mathrm{g} / \mathrm{L} \quad 10$ EPA Method 8260

$\mu \mathrm{g} / \mathrm{L} \quad 100$ Final PDWS (EPA, 1998a)

$\mu \mathrm{g} / \mathrm{L} \quad 100$ Final PDWS (EPA, 1998a)

$\mu \mathrm{g} / \mathrm{L} \quad 20$ EPA Method 8240

ug/L 10 Set by EPD/EMS

$\mu \mathrm{g} / \mathrm{L} 5 \mathrm{Final}$ PDWS (EPA, 1998a)

$\mu \mathrm{g} / \mathrm{L} \quad 50$ EPA Method 8240

$\mu \mathrm{g} / \mathrm{L} \quad 5 \quad$ Final PDWS (EPA, 1998a)

$\mu \mathrm{g} / \mathrm{L} \quad 2$ Final PDWS (EPA, 1998a)

$\mu \mathrm{g} / \mathrm{L} \quad 100$ Final PDWS (EPA, 1998a)

$\mu \mathrm{g} / \mathrm{L} \quad 162$ EPA Method 8270

$\mu \mathrm{g} / \mathrm{L} \quad 20 \quad$ EPA Method 8240

$\mu \mathrm{g} / \mathrm{L} \quad 2$ Final PDWS (EPA, 1998a)

$\mu \mathrm{g} / \mathrm{L} \quad 100$ Final PDWS (EPA, 1998a)

$\mu \mathrm{g} / \mathrm{L} \quad 20 \quad$ EPA Method 8240 


\begin{tabular}{|c|c|c|c|c|c|}
\hline$\underline{\text { CAS }}$ & Analyte Name & Synonym & Unit & $\underline{\text { Limi }}$ & tSource ${ }^{\dagger}$ \\
\hline 126998 & Chloroprene & & $\boldsymbol{\mu} \mathrm{g} / \mathbf{L}$ & 3330 & EPA Method 8240 \\
\hline 7440473 & Chromium, total recoverable & & $\mu \mathrm{g} / \mathrm{L}$ & 100 & Final PDWS (EPA, 1998a) \\
\hline 218019 & Chrysene & & $\mu \mathrm{g} / \mathrm{L}$ & 0.2 & Proposed PDWS (EPA, 1990) \\
\hline 156592 & cis-1,2-Dichloroethene & cis-1,2-Dichloroethylene & $\boldsymbol{\mu g} / \mathbf{L}$ & 70 & Final PDWS (EPA, 1998a) \\
\hline 10061015 & cis-1,3-Dichloropropene & & $\mu \mathrm{g} / \mathrm{L}$ & 20 & EPA Method 8240 \\
\hline 7440484 & Cobalt, total recoverable & & $\mu g / L$ & 100 & $\begin{array}{l}\text { EPA Method } 6010 \\
\text { Appendix I, per WSRC-RP- }\end{array}$ \\
\hline 7440508 & Copper, total recoverable & & $\mu \mathrm{g} / \mathrm{L}$ & 29.8 & $98-01263$ \\
\hline 57125 & Cyanide & & $\mu \mathrm{g} / \mathrm{L}$ & 200 & Final PDWS (EPA, 1998a) \\
\hline 319868 & delta-Benzene hexachloride & delta-BHC & $\mu \mathrm{g} / \mathrm{L}$ & 0.5 & EPA Method 8080 \\
\hline 2303164 & Diallate & & $\mu \mathrm{g} / \mathrm{L}$ & 162 & EPA Method 8270 \\
\hline 53703 & Dibenz $[\mathrm{a}, \mathrm{h}]$ anthracene & & $\mu \mathrm{g} / \mathrm{L}$ & 0.3 & Proposed PDWS (EPA, 1990) \\
\hline 132649 & Dibenzofuran & & $\mu \mathrm{g} / \mathrm{L}$ & 10 & EPA Method 8270 \\
\hline 124481 & Dibromochloromethane & & $\mu \mathrm{g} / \mathrm{L}$ & 100 & Final PDWS (EPA, 1998a) \\
\hline 74953 & Dibromomethane & Methylene bromide & $\mathbf{u g} / \mathbf{L}$ & 20 & EPA Method 8240 \\
\hline 75718 & Dichlorodifluoromethane & & $\mu \mathrm{g} / \mathrm{L}$ & 20 & EPA Method 8240 \\
\hline 75092 & Dichloromethane & Methylene Chloride & $\mu \mathrm{g} / \mathrm{L}$ & 5 & Final PDWS (EPA, 1998a) \\
\hline 60571 & Dieldrin & & $\mu \mathrm{g} / \mathrm{L}$ & 8.3 & EPA Method 8080 \\
\hline 84662 & Diethyl phthalate & & $\mathrm{ug} / \mathrm{L}$ & 10 & EPA Method $8270 \mathrm{C}$ \\
\hline 60515 & Dimethoate & & $\mu \mathrm{g} / \mathrm{L}$ & 162 & EPA Method 8270 \\
\hline 131113 & Dimethyl phthalate & & ug/L & 10 & EPA Method $8270 \mathrm{C}$ \\
\hline 84742 & Di-n-butyl phthalate & & $\mathrm{ug} / \mathrm{L}$ & 10 & EPA Method 8270C \\
\hline 117840 & Di-n-octyl phthalate & & $\mathrm{ug} / \mathrm{L}$ & 10 & EPA Method $8270 \mathrm{C}$ \\
\hline 122394 & Diphenylamine & & $\mu \mathrm{g} / \mathrm{L}$ & 162 & EPA Method 8270 \\
\hline 298044 & Disulfoton & & $\mu \mathrm{g} / \mathrm{L}$ & 162 & EPA Method 8270 \\
\hline 959988 & Endosulfan I & & $\mu \mathrm{g} / \mathrm{L}$ & 0.5 & EPA Method 8080 \\
\hline 33213659 & Endosulfan II & & $\mu \mathrm{g} / \mathrm{L}$ & 1.1 & EPA Method 8080 \\
\hline 1031078 & Endosulfan sulfate & & $\mu \mathrm{g} / \mathrm{L}$ & 1.1 & EPA Method 8080 \\
\hline 72208 & Endrin & & $\mu \mathrm{g} / \mathrm{L}$ & 2 & Final PDWS (EPA, 1998a) \\
\hline 7421934 & Endrin aldehyde & & $\mu \mathrm{g} / \mathrm{L}$ & 1.7 & EPA Method 8080 \\
\hline 97632 & Ethyl methacrylate & & $\mu \mathrm{g} / \mathrm{L}$ & 5 & EPA Method 8270 \\
\hline 62500 & Ethyl methanesulfonate & & $\mu \mathrm{g} / \mathrm{L}$ & 162 & EPA Method 8270 \\
\hline 100414 & Ethylbenzene & & $\mu \mathrm{g} / \mathrm{L}$ & 700 & Final PDWS (EPA, 1998a) \\
\hline 52857 & Famphur & & $\mu \mathrm{g} / \mathrm{L}$ & 162 & EPA Method 8270 \\
\hline 206440 & Fluoranthene & & $\mu \mathrm{g} / \mathrm{L}$ & 10.2 & EPA Method 8270 \\
\hline 86737 & Fluorene & & $\mu \mathrm{g} / \mathrm{L}$ & 10.2 & EPA Method 8270 \\
\hline 76448 & Heptachlor & & $\mu \mathrm{g} / \mathrm{L}$ & 0.4 & Final PDWS (EPA, 1998a) \\
\hline 1024573 & Heptachlor epoxide & & $\mu \mathrm{g} / \mathrm{L}$ & 0.2 & Final PDWS (EPA, 1998a) \\
\hline 118741 & Hexachlorobenzene & & $\mu \mathrm{g} / \mathrm{L}$ & 1 & Final PDWS (EPA, 1998a) \\
\hline 87683 & Hexachlorobutadiene & & $\mu \mathrm{g} / \mathrm{L}$ & 10 & EPA Method 8270 \\
\hline 77474 & Hexachlorocyclopentadiene & & $\mu \mathrm{g} / \mathrm{L}$ & 50 & Final PDWS (EPA, 1998a) \\
\hline 67721 & Hexachloroethane & & $\mu \mathrm{g} / \mathrm{L}$ & 1 & EPA Method 8270 \\
\hline 1888717 & Hexachloropropene & & $\mu \mathrm{g} / \mathrm{L}$ & 162 & EPA Method 8270 \\
\hline 193395 & Indeno $[1,2,3-\mathrm{c}, \mathrm{d}] \mathrm{pyrene}$ & & $\mu \mathrm{g} / \mathrm{L}$ & 1 & EPA Method 8270 \\
\hline 74884 & Iodomethane (Methyl iodide) & & $\mu g / L$ & 250 & EPA Method 8240 \\
\hline 78831 & Isobutyl alcohol & & $\mu \mathrm{g} / \mathrm{L}$ & 1669 & EPA Method 8240 \\
\hline 465736 & Isodrin & & $\mu \mathrm{g} / \mathrm{L}$ & 162 & EPA Method 8270 \\
\hline 78591 & Isophorone & & $\mu \mathrm{g} / \mathrm{L}$ & 10.2 & EPA Method 8270 \\
\hline 120581 & Isosafrole & & $\mu \mathrm{g} / \mathrm{L}$ & 162 & EPA Method 8270 \\
\hline 143500 & Kepone & & $\mu \mathrm{g} / \mathrm{L}$ & 162 & EPA Method 8270 \\
\hline
\end{tabular}




\section{CAS Analyte Name}

7439921 Lead, total recoverable

58899 Lindane

$108394 \quad \mathrm{~m}$-Cresol (3-Methylphenol)

7439976 Mercury, total recoverable

126987 Methacrylonitrile

91805 Methapyrilene

72435 Methoxychlor

78933

108101

80626

66273

99092

108383

91203

$\mathbf{7 4 4 0 0 2 0}$ Nickel, total recoverable

98953

55185

62759

924163

86306

621647

Methyl ethyl ketone

Methyl isobutyl ketone

Methyl methacrylate

Methyl methanesulfonate

m-Nitroaniline

m-Xylene

Naphthalene

N-Nitrosodipropylamine

10595956 N-Nitrosomethylethylamine

$100754 \quad$ N-Nitrosopiperidine

$930552 \quad$ N-Nitrosopyrrolidine

$126681 \quad \mathrm{O}, \mathrm{O}, \mathrm{O}-\mathrm{T}$ riethyl phosphorothioate

95487 o-Cresol (2-Methylphenol)

95534 o-Toluidine

95476 o-Xylene

72548

72559

50293

56382

298000

1336363

106445

60117

608935

82688

87865

$\begin{array}{ll} & \text { pH } \\ 62442 & \text { Phenacetin } \\ 85018 & \text { Phenanthrene } \\ 108952 & \text { Phenol } \\ 298022 & \text { Phorate } \\ 106503 & \text { p-Phenylenediamine } \\ 23950585 & \text { Pronamid } \\ 107120 & \text { Propionitrile } \\ 106423 & \text { p-Xylene } \\ 129000 & \text { Pyrene }\end{array}$

\section{Synonym}

gamma-BHC

\section{2-Butanone \\ 4-methyl-2-pentanone}

4,4-DDD
4,4-DDE
4,4-DDT

Polychlorinated Biphenyls

\section{Unit LimitSource $\dagger$}$$
\mu \mathrm{g} / \mathrm{L}
$$$$
18 \text { 98-01263 }
$$$$
\mu \mathrm{g} / \mathrm{L} \quad 0.2 \text { Final PDWS (EPA, 1998a) }
$$$$
\mu \mathrm{g} / \mathrm{L} \quad 100 \text { EPA Method } 8270
$$$$
\mu \mathrm{g} / \mathrm{L} \quad 2 \text { Final PDWS (EPA, 1998a) }
$$$$
\mu \mathrm{g} / \mathrm{L} \quad 833 \text { EPA Method } 8240
$$$$
\mu \mathrm{g} / \mathrm{L} \quad 162 \text { EPA Method } 8270
$$$$
\mu \mathrm{g} / \mathrm{L} \quad 40 \quad \text { Final PDWS (EPA, 1998a) }
$$$$
\text { ug/L } 20 \text { EPA Method 8260B }
$$$$
\mathrm{ug} / \mathrm{L} \quad 10 \text { EPA Method 8260B }
$$

$\mu \mathrm{g} / \mathrm{L} \quad 100$ EPA Method 8270

$\mu \mathrm{g} / \mathrm{L} \quad 162$ EPA Method 8270

$\mu \mathrm{g} / \mathrm{L} \quad 10 \quad$ EPA Method 8270

$\mu \mathrm{g} / \mathrm{L} \quad$ NA NA

$\mu \mathrm{g} / \mathrm{L} \quad 167$ EPA Method 8270

$\mu g / L \quad 100$ Final PDWS (EPA, 1998a)

$\mu \mathrm{g} / \mathrm{L} \quad 10.2$ EPA Method 8270

$\mu \mathrm{g} / \mathrm{L} \quad 162 \quad$ EPA Method 8270

$\mu \mathrm{g} / \mathrm{L} \quad 167$ EPA Method 8270

$\mu \mathrm{g} / \mathrm{L} \quad 162$ EPA Method 8270

$\mu \mathrm{g} / \mathrm{L} \quad 10.2$ EPA Method 8270

$\mu \mathrm{g} / \mathrm{L} \quad 10.2$ EPA Method 8270

$\mu \mathrm{g} / \mathrm{L} \quad 162$ EPA Method 8270

$\mu \mathrm{g} / \mathrm{L} \quad 162$ EPA Method 8270

$\mu \mathrm{g} / \mathrm{L} \quad 162$ EPA Method 8270

$\mu \mathrm{g} / \mathrm{L} \quad 162$ EPA Method 8270

$\mu \mathrm{g} / \mathrm{L} \quad 10 \quad$ EPA Method 8270

$\mu \mathrm{g} / \mathrm{L} \quad 162$ EPA Method 8270

$\mu \mathrm{g} / \mathrm{L} \quad 10 \quad$ EPA Method 8260

$\mu \mathrm{g} / \mathrm{L} \quad 1.1 \quad$ EPA Method 8080

$\mu \mathrm{g} / \mathrm{L} \quad 0.5 \quad$ EPA Method 8080

g/L $\quad 1.7$ EPA Method 8080

$\mu \mathrm{g} / \mathrm{L} \quad 0.8 \quad$ EPA Method 8080

$\mu \mathrm{g} / \mathrm{L} \quad 0.8 \quad$ EPA Method 8080

NA NA

$\mu \mathrm{g} / \mathrm{L} \quad 120$ EPA Method 8270

$\mu \mathrm{g} / \mathrm{L} \quad 162$ EPA Method 8270

$\mu \mathrm{g} / \mathrm{L} \quad 162$ EPA Method 8270

$\mu \mathrm{g} / \mathrm{L} \quad 162$ EPA Method 8270

$\mu \mathrm{g} / \mathrm{L} \quad 1$ Final PDWS (EPA, 1998a)

4.11 - Appendix I, per WSRC-RP-

pH 6-15 98-01263

$\mathrm{g} / \mathrm{L} \quad 162$ EPA Method 8270

$\mu \mathrm{g} / \mathrm{L} \quad 10.2$ EPA Method 8270

$\mu \mathrm{g} / \mathrm{L} \quad 167$ EPA Method 8270

$\mu \mathrm{g} / \mathrm{L} \quad 1.7$ EPA Method 8080

$\mu \mathrm{g} / \mathrm{L} \quad 162$ EPA Method 8270

$\mu \mathrm{g} / \mathrm{L} \quad 162$ EPA Method 8270

$\mu \mathrm{g} / \mathrm{L} \quad 3330$ EPA Method 8240

NA NA

$\mu \mathrm{g} / \mathrm{L} \quad 10.2$ EPA Method 8270

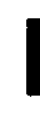


CAS

94597

7782492 Selenium, total recoverable

$\mathbf{7 4 4 0 2 2 4}$ Silver, total recoverable

93721

Silvex

Specific Conductance

100425 Styrene

18496258 Sulfide

127184 Tetrachloroethylene

$\mathbf{7 4 4 0 2 8 0}$ Thallium, total recoverable

297972 Thionazin

7440315 Tin

108883 Toluene

8001352 Toxaphene

156605 trans-1,2-Dichloroethylene

10061026 trans-1,3-Dichloropropene

110576 trans-1,4-Dichloro-2-butene

79016 Trichloroethylene

75694 Trichlorofluoromethane

7440622 Vanadium

108054 Vinyl acetate

1330207 Xylenes

7440666 Zinc, total recoverable

\section{Synonym}

2,4,5-TP (Silvex)

0,0-Diethyl 0-2-Pyrazinyl

Phosphorothioate

Xylene (Total)

\begin{tabular}{|c|c|c|}
\hline & Lin & \\
\hline$\overline{\mathrm{gg} / \mathrm{L}}$ & 162 & EPA Method 8270 \\
\hline $\mathrm{lg} / \mathrm{L}$ & 50 & Final PDWS (EPA, 1998a) \\
\hline $1 \mathrm{~g} / \mathrm{L}$ & 20 & EPA Method 6010B \\
\hline $\mathrm{g} / \mathrm{L}$ & 50 & Final PDWS (EPA, 1998a) \\
\hline & 10.6 & Appendix I, per WSRC-RP- \\
\hline$/ \mathbf{c m}$ & 37.0 & $98-01263$ \\
\hline $\mathrm{g} / \mathrm{L}$ & 100 & Final PDWS (EPA, 1998a) \\
\hline $\mathrm{g} / \mathrm{L}$ & 16700 & EPA Method 9030 \\
\hline$g / L$ & 5 & Final PDWS (EPA, 1998a) \\
\hline$g / L$ & 2 & Final PDWS (EPA, 1998a) \\
\hline$\mu \mathrm{g} / \mathrm{L}$ & 162 & EPA Method 8270 \\
\hline$\mu \mathrm{g} / \mathrm{L}$ & 500 & EPA Method 282.2 \\
\hline $\mathrm{lg} / \mathrm{L}$ & 1000 & Final PDWS (EPA, 1998a) \\
\hline $4 \mathrm{~g} / \mathrm{L}$ & 3 & Final PDWS (EPA, 1998a) \\
\hline$\mu \mathrm{g} / \mathrm{L}$ & 100 & Final PDWS (EPA, 1998a) \\
\hline $1 \mathrm{~g} / \mathrm{L}$ & 20 & EPA Method 8240 \\
\hline $\mathrm{g} / \mathrm{L}$ & 500 & EPA Method 8240 \\
\hline$\mu \mathrm{g} / \mathrm{L}$ & 5 & Final PDWS (EPA, 1998a) \\
\hline$\mu \mathrm{g} / \mathrm{L}$ & 20 & EPA Method 8240 \\
\hline$\mu \mathrm{g} / \mathrm{L}$ & 133 & EPA Method 6010 \\
\hline$\mu \mathrm{g} / \mathrm{L}$ & 100 & EPA Method 8240 \\
\hline$\mu \mathrm{g} / 1$ & 10000 & Final PDWS (EPA 1998a) \\
\hline $\mathrm{g} /$ & 29.3 & $\begin{array}{l}\text { Appendix I, per WSRC-R } \\
98-01263\end{array}$ \\
\hline
\end{tabular}

$\dagger \quad$ Practical Quantitation Limits (PQLs) are provided as limits where EPA methods are listed as the "Source". References for dated sources are in the References section.

Note: Analytes from the Appendix I constituent list of the approved Closure/Post-Closure Plan, Q-CLP-G00008, Rev. 2, October 26, 1998 (WSRC-RP-98-01263) are shown in bold text. Analytes from the Appendix II list of the closure plan include all the analytes listed above with the exception of $\mathrm{pH}$ and specific conductance, which are only listed in Appendix I.

\section{References}

EPA (U.S. Environmental Protection Agency), 1977. National Interim Primary Drinking Water Regulations, EPA-570/9-76-003. Washington, DC.

EPA (U.S. Environmental Protection Agency), 1986. Water Pollution Control; National Primary Drinking Water Regulations, Radionuclides (Proposed). Federal Register, September 30, 1986, pp. 34835-34862. Washington, DC.

EPA (U.S. Environmental Protection Agency), 1990. National Primary and Secondary Drinking Water Regulations; Synthetic Organic Chemicals and Inorganic Chemicals (Proposed Rule). Federal Register, July 25, 1990, pp. 303690448. Washington, DC.

EPA (U.S. Environmental Protection Agency), 1991. National Primary Drinking Water Regulations; Radionuclides; Proposed Rule. Federal Register, July 18, 1991, pp. 33052-33127. Washington, DC.

EPA (U.S. Environmental Protection Agency), 1993a. National Primary Drinking Water Regulations. Code of Federal Regulations, Section 40, Part 141, pp. 592-732. Washington, DC.

EPA (U.S. Environmental Protection Agency), 1993b. National Secondary Drinking Water Regulations. Code of Federal Regulations, Section 40, Part 143, pp. 774-777. Washington, DC. 
SCDHEC (South Carolina Department of Health and Environmental Control), 1981. State Primary Drinking Water Regulations, R.61-58.5. Columbia, SC.

EPA (U.S. Environmental Protection Agency), 1977. National Interim Primary Drinking Water Regulations, EPA-570/9-76-003. Washington, DC.

EPA (U.S. Environmental Protection Agency), 1993. National Primary Drinking Water Regulations, Code of Federal Regulations, Section 40, Part 141, pp. 592-732. Washington, DC.

SCDHEC (South Carolina Department of Health and Environmental Control), 1981. State Primary Drinking Water Regulations, R.61-58.5. Columbia, SC.

SRS (Westinghouse Savannah River Site), 1998. Closure/Post-Closure Plan, Q-CLP-G-00008, Rev. 2, October 26, 1998 (WSRC-RP-98-01263). Aiken, SC. 


\section{Appendix B}

Groundwater Monitoring Results Tables 


\section{Key to Reading the Tables}

The following abbreviations may appear in the data tables:

\section{Constituents}

1,2,3,4,6,7,8-HPCDD
1,2,3,4,6,7,8-HPCDF
1,2,3,4,7,8-HXCDD
1,2,3,4,7,8-HXCDF
Lindane
PCB

$1,2,3,7,8-\mathrm{PCDD}$

1,2,3,7,8-PCDF

Sp. conductance

TCDD

TCDF

\section{Laboratories}

CN

EM

GE and GP

$\mathrm{SC}$

$\mathrm{SP}$

TM

WA and WS

\section{Nomenclature}

AZ

CBAU

CBCU

GAU

GCCZ

LAZ_UTRA

LLAZ

LLLAZ

MAAZ

$M S A Z$ CBCU

PZ

UAZ_UTRA

ULLAZ

UNKNOWN
-

CBA

$\mathrm{CBC}$

GAU

GC

LAZ

LLAZ

LL.

$M$

MCBC

$\mathrm{PZ}$

UAZ

UL

UNK 1,2,3,4,6,7,8-heptachlorodibenzo-p-dioxin

1,2,3,4,6,7,8-heptachlorodibenzo-p-furan

$1,2,3,4,7,8$-hexachlorodibenzo-p-dioxin

1,2,3,4,7,8-hexachlorodibenzo-p-furan

gamma-benzene hexachloride

polychlorinated biphenyl

1,2,3,7,8-pentachlorodibenzo-p-dioxin

1,2,3,7,8-pentachlorodibenzo-p-furan

specific conductance

tetrachlorodibenzo-p-dioxin

tetrachlorodibenzo-p-furan

Clemson Technical Center, Inc.

Environmental Protection Department/Environmental Monitoring Section (EPD/EMS) Laboratory

General Engineering Laboratories, Inc.

Savannah River Technology Center

Spencer Testing Services, Inc.

Thermo NUtech

Recra LabNet Philadeiphia.

Aquifer Zone

Crouch Branch Aquifer Unit (previously Black Creek)

Crouch Branch Confining Unit

Gordon Aquifer Unit

Green Clay Confining Zone

Lower Aquifer Zone-Upper Three Runs Aquifer Unit

Lost Lake Aquifer Zone

Lower Lost Lake Aquifer Zone (previously Lower Congaree)

M-Area Aquifer Zone (previously Water Table)

Middle Sand Aquifer Zone-CBCU (previously Ellenton Sand)

Perched Zone

Upper Aquifer Zone-Upper Three Runs Aquifer Unit

Upper Lost Lake Aquifer Zone (previously Upper Congaree)

Unknown 


\section{Sampling Codes}

B

C

D

E

I

L

N

$P$

$S$

$\mathrm{T}$

W

$x$

\section{Sampling Methods}

B

O

$P$

S

V

\section{Units}

$\mathrm{mg} / \mathrm{L}$

$\mathrm{msl}$

MSL

NTU

pCi/L

$\mathrm{pCi} / \mathrm{mL}$

$\mathrm{pH}$

$\mu g / L$

$\mu \mathrm{S} / \mathrm{cm}$ blank sample was collected

well was pumping continuously

well was dry

equipment blank was collected

well went dry during sampling; insufficient water to collect all samples

well went dry before sampling began; only depth to water can be determined

well was not stabilized before sampling began

inaccessibility or mechanical failure prevented sample collection and field analysis of the water

no water in standpipe

samples were collected, but some samples were not sent to the laboratory due to high turbidity

unable to sample well because of stabilization or sampling equipment failure; water-level measurements were obtained well went dry during purging; samples collected after well recovered

sample collected using an open-bucket bailer sample collected by method other than bailer or pump sample collected using a bladder pump sample collected using a single-speed centrifugal downhole pump sample collected using a variable-speed pump

milligrams per liter

mean sea level

million structures per liter nephelometric turbidity unit picocuries per liter picocuries per milliliter $\mathrm{pH}$ unit micrograms per liter microsiemens per centimeter 
Other Codes

$\begin{array}{ll}\text { CLP } & \text { EPA Functional Guideline Codes } \\ \text { CS } & \text { carbon steel } \\ \text { DF } & \text { dilution factor column in data tables } \\ \text { E } & \text { exponential notation }\left(e . g ., 1.1 \mathrm{E}-09=1.1 \times 10^{-9}=0.0000000011\right) \\ \text { STORET } & \text { EPA STORET result qualifiers } \\ \text { Filt. } & \text { Data results after application of the Data Usability filter } \\ \text { GWPS } & \text { groundwater protection standard } \\ \text { MCS } & \text { monitoring coristituents standard } \\ \text { Mod } & \text { modifier column in data tables } \\ \text { NDD } & \text { "not decision data" } \\ \text { PDWS } & \text { primary drinking water standard } \\ \text { PVC } & \text { polywinyl chloride } \\ \text { ST } & \text { exceeded the GWPS column in data tables } \\ \text { TOC } & \text { top of casing } \\ \text { KEQL } & \text { less than the sample-specific estimated quantitation limit }\end{array}$

\section{Results Below Detection}

For radiological analyses, the analytical result field contains the result recorded on the analytical instrument and reported by the laboratory, even if it is negative. For nonradiological analyses, if the analyte is not detected, the sample-specific estimated quantitation limit (EQL) is entered into the result field and is reported with a less than $[<]$ sign. The EQL is defined as the lowest concentration that can be achieved reliably within specified limits of precision and accuracy during routine laboratory operating conditions. The sample-specific EQL is modified for sample concentration or dilution or unusual aliquot size that affects analytical sensitivity.

\section{Uncertainty and Data Usability}

In April 1998, SCDHEC accepted guidance proposed by SRS to apply a method for minimizing uncertainty in compliance decisions potentially affecting long-term monitoring or remediation (letter, Taylor to Cook; April 21, 1998). The method is applied by processing or "filtering" the data, using the EPA Functional Guideline Codes applied by the laboratories to qualify the analytical results. By removing all data with a result qualifier of " $L$ ", " $R$ ", " $U$ ", and " $J$ " from consideration, groundwater data users can ensure that only quantified numerical results are applied to the decision process. The output of the filtering process populates the "Filt" column as follows:

1) "Null" or "blank" - Data not remarked. The analytical result is acceptable for use as reported, and the result is not greater than an associated concentration limit for the analyte. If a concentration limit exists for the analyte, and the result is greater than the concentration limit, then the "Filt." Column will contain the applicable limit, and the "greater than" symbol. For example, in the case of trichloroethylene, " $>5$ " would indicate the result exceeded a concentration limit of 5 ug/L.

Rationale: The best result would be one without qualifiers, so the preferred choice would be the maximum result that did not have any qualifiers.

2) "J", "L", "N", "NJ", or "JL" - "J" identifies that the analyte was positively identified; the associated numerical value is an estimated concentration of the analyte in the sample. "L" Indicates the sample result is off scale high. "JL" Indicates an estimated quantity of a sample that is off scale high. " $\mathrm{N}$ " is used for all TIC (tentatively identified compounds) and indicates the presence of an analyte for which there is presumptive evidence to make a tentative identification. "NJ" means the presence of an analyte that has been tentatively identified and the associated numerical value represents its approximate concentration. 
Rationale: an estimate can still provide useful information. Although there may be a range of uncertainty around the actual value, the value itself may still grossly exceed a regulatory standard. However, a estimated value is less certain than an unqualified result. Therefore, this would be labeled as "NDD" (not decision data).

3) "U" - material analyzed for, but not detected. The analyte concentration is less than the sample specific Estimated Quantitation Limit and labeled "<EQL".

Rationale: a result above the detection limit would be chosen before a result below detection so that the process is not biased toward false negatives.

4) "UJ" - result is not above the reported sample quantitation limit, but the reported quantitation limit itself is approximate, and may not represent the actual limit of quantitation necessary to accurately and precisely measure the analyte in the sample.

Rationale: the additional qualifiers make this result less reliable for use than the " $U$ " without qualifiers. These results would be labeled "<EQL".

5) "Rejected" - The sample results are rejected due to serious deficiencies in the ability to analyze the sample and meet quality control criteria. The presence or absence of the analyte cannot be verified.

Rationale: the only value in providing this result in the report is to indicate that the lab attempted to analyze the sample. If there are any other results available, the result with the "R" qualifier should not be reported. If it is reported, it is definitely "NDD" (not decision data).

\section{Holding Times}

Standard analytical methods include a limit, called holding time, on the maximum elapsed time between sample collection and extraction or analysis by the laboratory. In the data tables, the result qualifier $Q$ in the "EPA" column indicates that holding time was exceeded. Analyses performed beyond holding times may not yield valid results.

The South Carolina Department of Health and Environmental Control (SCDHEC) allows only 15 minutes to elapse between sampling and analysis for $\mathrm{pH}$. Thus, only field $\mathrm{pH}$ measurements can meet the holding time criterion; laboratory $\mathrm{pH}$ analyses always will exceed it.

The laboratory procedure used for the determination of specific conductance allows one day to elapse between sampling and analysis. Thus, laboratory specific conductance measurements may exceed the holding time criterion.

\section{Data Qualification}

The contract laboratories submit sample- or batch-specific quality assurance/quality control information either at the same time as analytical results or in a quarterly summary. Properly defined and used, data qualifiers can be a key component in assessing data usability. The EPA Functional Guideline Codes used by the analytical laboratories are shown in the CLP result qualifier column are defined below. These modifiers appear in the data tables under the column $C L P$. EPA STORET codes appear in the data tables under the column labeled EPA. 
(Blank) Data not remarked. The analytical result is acceptable for use as reported.

$J \quad$ The analyte was positively identified; the associated numerical value is an estimated concentration of the analyte in the sample.

$N \quad$ The analysis indicates the presence of an analyte for which there is presumptive evidence to make a tentative identification. Used for all TIC results.

$R \quad$ The sample results are rejected due to serious deficiencies in the ability to analyze the sample and meet quality control criteria. The presence of absence of the analyte cannot be verified. Assignment of $R$ requires approval by the appropriate WSRC data validation coordinator.

$U \quad$ Material analyzed for but not detected. Analytical result reported is less than the sample quantitation limit.

NJ The analysis indicates the presence of an analyte that has been tentatively identified and the associated numerical value represents its approximate concentration.

Note: These are only some of the qualifiers present in the database. All modifiers associated with the data are published in the result tables of EPD/EMS' quarterly groundwater monitoring reports, the official repository of the data.

\title{
"EPA" Qualifiers - EPA STORET Codes
}

\author{
(Blank) Data not remarked \\ C The result is calculated. \\ 1 The result is less than the ssEQL, but equal to or greater than the MDL. Always \\ $K \quad$ reported with an associated EPA functional Guideline Code qualifier of \\ $L \quad$ The actual concentration is known to be less than the reported result. \\ $O \quad$ Sample received by laboratory, but the analysis was lost or not performed. \\ $Q \quad$ Sample was held beyond normal holding time prior to analysis.
}

Note: These are only some of the qualifiers present in the database. All modifiers associated with the data are published in the result tables of EPD/EMS' quarterly groundwater monitoring reports, the official repository of the data. 
Table B. Assessment Monitoring Results for Groundwater Wells

WELL LFW 28

\section{SRS Coord. \\ N 86079.6 \\ E 45555.3

\author{
Lat/Longitude \\ 33.29048 Deg $N$ \\ -81.7141 Deg $W$
}

SAMPLE DATE

\section{FIELD DATA}

\section{Parameter}

Water Elevation

$\mathrm{pH}$

Sp. Conductance

Water temperature

Alkalinity as $\mathrm{CaCO} 3$

Turbidity

Volumes purged

Sampling code

\section{ANALYTICAL DATA}

Groundwater Protection Standard

\section{ST Parameter}

1,1,1,2-Tetrachloroethane

1,1,1-Trichloroethane

1,1,2,2-Tetrachloroethane

1,1,2-Trichloroethane

1,1-Dichloroethane

1,1-Dichloroethylene

1,1-Dichloropropene

1,2,3-Trichloropropane

1,2,4,5-Tetrachlorobenzene

1,2,4-Trichlorobenzene

1,2-Dibromo-3-chloropropane

1,2-Dibromoethane

1,2-Dichlorobenzene

1,2-Dichloroethane

1,2-Dichloropropane

1,3,5-Trinitrobenzene

1,3-Dichlorobenzene

1,3-Dichloropropane

1,3-Dinitrobenzene

1,4-Dichlorobenzene

1,4-Naphthoquinone

1-Naphthylamine

2,2-Dichjoropropane

2,2-Oxybis(1-chloropropane)

2,3,4,6-Tetrachiorophenol

$2,4,5-T$

2,4,5-TP (Silvex)

2,4,5-Trichlorophenol

2,4,6-Trichlorophenol

2,4-Dichlorophenol

2,4-Dichlorophenoxyacetic acid

2,4-Dimethyl phenol

2,4-Dinitrophenol

2,4-Dinitrotoluene

2,6-Dichlorophenol

2,6-Dinitrotoluene

2-Acetylaminofluorene

2-Chloronaphthalene

2-Chlorophenol

2-Hexanone

2-Methyl-4,6-dinitrophenol

2-Methylnaphthalene

2-Naphthylamine

2-Nitrophenol

2-sec-Butyl-4,6-dinitrophenol

3,3 "-Dichlorobenzidine

3,3"-Dimethylbenzidine

3-Methylcholanthrene

4-Aminobiphenyl

4-Bromophenyl phenyl ether

4-Chloro-m-cresol

\author{
Screen Zone Elevation \\ $162.1-141.1 \mathrm{ft} \mathrm{msl}$
}

$03 / 08 / 00$

\begin{tabular}{l} 
1st Half \\
\hline 161.9 \\
5.2 \\
22 \\
20.4 \\
5 \\
1.3 \\
2.81
\end{tabular}

1st Half
$<5$
$<5$
$<5$
$<5$
$<5$
$<5$
$<5$
$<5$

$<10$

$<5$

$<5$

$<5$

$<5$
$<5$

$<5$

$<5$

$\begin{array}{cc}\text { CLP EPA } & \text { Filit. } \\ U & <E Q L \\ U & <E Q L \\ U & <E Q L \\ U & <E Q L \\ U & <E Q L \\ U & <E Q L \\ U & <E Q L \\ U & <E Q L\end{array}$

$<$ EQL

$<E Q L$

$<E Q L$

$<E Q L$

$<$ EOL

$<E Q L$

$<E Q L$

$<$ EQL

$<$ EQL

$<$ EQL

U

$<20$

U

$<E Q L$

$<20$

u

$<E Q L$

ug/h

Notes: Concentrations in bold italics exceed the groundwater protection standards listed in Appendix A. Bold italics were not assigned if the result was qualified with a "J", "R", "L", or "U" qualifier.

$+=$ exceeded the Groundwater Protection Standards listed in Appendix A. 
Table B. Assessment Monitoring Results for Groundwater Wells (Cont.)

\section{WELL LFW 28}

ANALYTICAL DATA

Groundwater Protection Standard

ST Parameter

2nd Half CLP EPA Filt. Unit

4-Chioroaniline

4-Chlorophenyl phenyl ether

4-Nitrophenol

5-Nitro-o-toluidine

7.12-Dimethylbenz(a)anthracene

Acenaphthene

Acenaphthylene

Acetone

Acetonitrile (Methyl cyanide)

Acetophenone

Acrolein

Acrylonitrite

Aldrin

Allyl chloride

Anthracene

Antimony, total recoverable

Arsenic, total recoverable

Barium, total recoverable

Benzene

Benzo(a)anthracene

Benzo(a)pyrene

Benzo(b)fluoranthene

Benzo(g,h,i)perylene

Benzo(k)fluoranthene

Benzyl alcohol

Beryllium, total recoverable

Bis(2-chloroethoxy) methane

Bis(2-chloroethyl) ether

Bis(2-ethylhexyl) phthalate

Bromochloromethane

Bromodichloromethane

Bromoform

Bromomethane (Methyl bromide)

Butylbenzyl phthalate

Cadmium, total recoverable

Carbon disulfide

Carbon tetrachloride

Chlorobenzene

Chiorobenzilate

Chloroethane

Chloroethene (Vinyl chloride)

Chloroform

Chloromethane (Methyl chloride)

Chloroprene

Chromium, total recoverable

Chrysene

Cobalt, total recoverable

Copper, total recoverable

Cyanide

Di-n-butyl phthalate

Di-n-octyl phthalate

Diallate

Dibenz(a,h)anthracene

Dibenzofuran

Dibromochloromethane

Dibromomethane (Methylene bromide)

Dichlorodifluoromethane

Dichloromethane (Methylene chloride)

Dieldrin

Diethyl phthalate

Dimethoate

Dimethyl phthalate

Diphenylamine

Disulfoton

Endosulfan I

Endosulfan II

Endosulfan sulfate

Endrin

\begin{tabular}{|c|c|c|c|}
\hline .94 & $\mathrm{~J}$ & 1 & NDD \\
\hline$<5$ & U & & $<E Q L$ \\
\hline$<5$ & $U$ & & $<E Q L$ \\
\hline$<5$ & $\mathrm{U}$ & & $<\mathrm{EQL}$ \\
\hline$<5$ & $\mathrm{U}$ & & $<E Q L$ \\
\hline$<10$ & $U$ & & $<E Q L$ \\
\hline$<5$ & $\mathrm{U}$ & & $<E Q L$ \\
\hline$<5$ & $\mathrm{U}$ & & $<E Q L$ \\
\hline$<5$ & $\mathrm{U}$ & & $<E Q L$ \\
\hline$<5$ & $U$ & & $<E Q L$ \\
\hline$<5$ & $U$ & & $<E Q L$ \\
\hline$<5$ & $\mathrm{U}$ & & $<E Q L$ \\
\hline$<5$ & U & & $<E Q L$ \\
\hline$<20$ & U & & $<E Q L$ \\
\hline$<10$ & $\mathrm{U}$ & & $<E Q L$ \\
\hline$<20$ & $\mathrm{U}$ & & $<E Q L$ \\
\hline 16.9 & $\mathbf{J}$ & 1 & NDD \\
\hline$<11$ & JU & $Q$ & $<\mathrm{EQL}$ \\
\hline
\end{tabular}

\begin{tabular}{|c|c|c|}
\hline$<20$ & $\mathrm{U}$ & $<E Q L$ \\
\hline$<200$ & U & $<E Q L$ \\
\hline$<50$ & $u^{\prime}$ & $<E C$ \\
\hline$<10$ & $\mathrm{U}$ & $<\mathrm{E}$ \\
\hline$<5$ & $\mathrm{U}$ & $<\mathrm{E}$ \\
\hline$<100$ & $\mathbf{U}$ & $<\mathrm{EC}$ \\
\hline$<3.21$ & JU & EC \\
\hline 10.7 & & $<20$ \\
\hline$<5$ & $\mathbf{U}$ & $<E C$ \\
\hline
\end{tabular}

ug/L

$\begin{array}{lll}<20 & U & <E Q L \\ <200 & U & <E Q L \\ <50 & U & <E Q L \\ <10 & U & <E Q L \\ & & \\ <5 & U & <E Q L \\ <100 & U & <E Q L \\ <10 & U & <E Q L \\ 15.1 & & <2000 \\ <5 & U & <E Q L\end{array}$

EQL

ug/L

ug/L

ug/L

ug/L

$\mathrm{ug} / \mathrm{L}$

ug/L

$\mathrm{ug} / \mathrm{L}$

$\mathrm{ug} / \mathrm{L}$

$<11$ JU $Q<E Q L$

$<$ EQL $\quad u g / L$

otes: Concentrations in bold italics exceed the groundwater protection standards listed in Appendix A. Bold italics were not assigned if the result was qualified with a "J", "R", "L", or "U" qualifier.

$+=$ exceeded the Groundwater Protection Standards listed in Appendix A. 
Table B. Assessment Monitoring Results for Groundwater Wells (Cont.)

WELL LFW 28

ANALYTICAL DATA

Groundwater Protection Standard

ST Parameter

1st Half CLP EPA Fitt.

Endrin aldehyde

Ethyl methacrylate

Ethyl methanesulfonate

Ethylbenzene

Famphur

Fluoranthene

Fluorene

Heptachlor

Heptachlor epoxide

Hexachlorobenzene

Hexachlorobutadiene

Hexachlorocyclopentadiene

Hexachloroethane

Hexachloropropene

Indeno(1,2,3-c,d)pyrene

lodomethane (Methyl iodide)

Isobutyl alcohol

Isodrin

isophorone

sosafrole

Kepone

Lead, total recoverable

Lindane

Mercury, total recoverable

Methacrylonitrile

Methapyrilene

Methoxychlor

Methyl ethyl ketone

Methyl isobutyl ketone

Methyl methacrylate

Methyl methanesulfonate

$\mathrm{N}$-Nitrosodi-n-butylamine

$\mathrm{N}$-Nitrosodiethylamine

$\mathrm{N}$-Nitrosodimethylamine

N-Nitrosodiphenylamine

$\mathrm{N}$-Nitrosodipropylamine

$\mathrm{N}$-Nitrosomethylethylamine

$\mathrm{N}$-Nitrosopiperidine

$\mathrm{N}$-Nitrosopyrrolidine

Naphthalene

Nickel, total recoverable

Nitrobenzene

O,O,O-Triethyl phosphorothioate

PCB

PCB 1016

PCB 1232

PCB 1242

PCB 1248

PCB 1254

PCB 1260

Parathion

Parathion methyl

Pentachlorobenzene

Pentachloronitrobenzene

Pentachloropheno

Phenacetin

Phenanthrene

Phenol

Phorate

Pronamid

Propionitrile

Pyrene

Safrole

Selenium, total recoverable

Silver, total recoverable

Specific conductance

Styrene

Sulfide

$\begin{array}{llll}<5 & \mathrm{U} & <\mathrm{EQL} \\ <5 & \mathrm{U} & <E Q L\end{array}$

$<5 \quad U \quad<E Q L$

$<500 \quad U<E Q$

3.39 J I NDD

$<.5 \quad U \quad<$ EQL

$<20 \quad U<E Q$

$<10 \quad U \quad<E Q L$

$<20 \quad U<E Q L$

$<50$

U

$<$ EQL

$<50$

U

$<E Q L$

ug/L

Notes: Concentrations in bold italics exceed the groundwater protection standards listed in Appendix A. Bold italics were not assigned if the result was qualified with a "J", "R", "L", or "U" qualifier.

$+=$ exceeded the Groundwater Protection Standards listed in Appendix A. 
Table B. Assessment Monitoring Results for Groundwater Wells (Cont.)

WELL LFW 28

ANALYTICAL DATA

Groundwater Protection Standard

\section{ST Parameter}

Tetrachloroethylene

Thallium, total recoverable

Thionazin

Tin, total recoverable

Toluene

Toxaphene

Trichloroethylene

Trichlorofluoromethane

Vanadium, total recoverable

Vinyl acetate

Xylenes

Zinc, total recoverable

alpha-Benzene hexachloride

alpha-Chlordane

beta-Benzene hexachloride

cis-1,2-Dichloroethylene

cis-1,3-Dichloropropene

delta-Benzene hexachloride

gamma-Chlordane

m-Cresol (3-Methyiphenol)

m-Nitroaniline

o-Cresol (2-Methylphenol)

o-Nitroaniline

o-Toluidine

p.p"-DDD

D.P"-DDE

$p, p "$-DDT

p-Cresol (4-Methylphenol)

p-Dimethylaminoazobenzene

p-Nitroaniline

p-Phenylenediamine

$\mathrm{pH}$

trans-1,2-Dichloroethylene

trans-1,3-Dichloropropene

trans-1,4-Dichloro-2-butene

\begin{tabular}{|c|c|c|c|}
\hline 1 st Half & CLP & EPA & Filt. \\
\hline$<5$ & $U$ & & $<\mathrm{EQL}$ \\
\hline$<10$ & $U$ & & $<E Q L$ \\
\hline$<200$ & U & & $<\mathrm{EQL}$ \\
\hline$<5$ & $u$ & & $<E Q L$ \\
\hline$<5$ & U & & $<E Q L$ \\
\hline$<5$ & U & & $<E Q L$ \\
\hline$<10$ & U & & $<E Q L$ \\
\hline$<5$ & U & & $<\mathrm{EQL}$ \\
\hline$<10$ & $U$ & & $<E Q L$ \\
\hline 14 & $\mathrm{~J}$ & I & NDD \\
\hline$<.1$ & $U$ & & $<E Q L$ \\
\hline$<5$ & U & & $<\mathrm{EQL}$ \\
\hline$<5$ & u & & $<\mathrm{EQL}$ \\
\hline
\end{tabular}

\begin{tabular}{|c|c|c|c|}
\hline 2nd Half & CLP & EPA & Filt. \\
\hline$<5$ & U & & $<E Q L$ \\
\hline 6.81 & J & I & NDD \\
\hline$<5$ & u & & $<\mathrm{EQL}$ \\
\hline$<5$ & U & & $<E Q L$ \\
\hline$<5$ & U & & $<E Q L$ \\
\hline$<10$ & U & & $<E Q L$ \\
\hline$<5$ & U & & $<E Q L$ \\
\hline$<10$ & U & & $<\mathrm{EQL}$ \\
\hline$<20$ & U & & $<$ EQL \\
\hline$<.095$ & $u$ & & $<E Q L$ \\
\hline$<5$ & U & & $<\mathrm{EQL}$ \\
\hline$<5$ & U & & $<E Q L$ \\
\hline
\end{tabular}

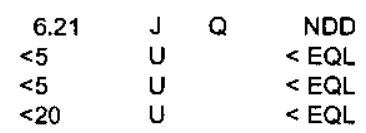

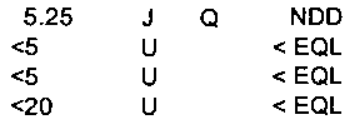

$\mathrm{pH}$
$\mathrm{ug} / \mathrm{h}$
$\mathrm{ug} / \mathrm{h}$
$\mathrm{ug} / \mathrm{h}$ 
Table B. Assessment Monitoring Results for Groundwater Wells (Cont.)

\section{WELL LFW 30}

$\begin{array}{ll}\text { SRS Coord. } & \text { Latlonqitude } \\ \text { N } 86318.4 & \text { 33.29038 Deg N } \\ \text { E 45170.9 } & -81.7155 \text { Deg W }\end{array}$

SAMPLE DATE

FIELD DATA

Parameter
Water Elevation
pH
Sp. Conductance
Water temperature
Alkalinity as $\mathrm{CaCO} 3$
Turbidity
Volumes purged
Sampling code

\section{ANALYTICAL DATA}

Groundwater Protection Standard

SI Parameter

1,1,1,2-Tetrachloroethane

1,1,1-Trichloroethane

1,1,2,2-Tetrachloroethane

1,1,2-Trichloroethane

1,1-Dichloroethane

1,1-Dichloroethylene

1,1-Dichloropropene

1,2,3-Trichloropropane

$1,2,4,5$-Tetrachlorobenzene

1,2,4-Trichlorobenzene

1,2-Dibromo-3-chloropropane

1,2-Dibromoethane

1,2-Dichlorobenzene

1,2-Dichloroethane

1,2-Dichloropropane

1,3,5-Trinitrobenzene

1,3-Dichlorobenzene

1,3-Dichloropropane

1,3-Dinitrobenzene

1,4-Dichlorobenzene

1,4-Naphthoquinone

1-Naphthylamine

2,2-Dichloropropane

2,2-Oxybis(1-chloropropane)

2,3,4,6-Tetrachlorophenol

$2,4,5-T$

2,4,5-TP (Silvex)

2,4,5-Trichlorophenol

2,4,6-Trichlorophenol

2,4-Dichlorophenol

2,4-Dichlorophenoxyacetic acid

2,4-Dimethyl phenol

2,4-Dinitrophenol

2,4-Dinitrotoluene

2,6-Dichloropheno

2,6-Dinitrotoluene

2-Acetylaminofluorene

2-Chloronaphthalene

2-Chlorophenol

2-Hexanone

2-Methyl-4,6-dinitrophenol

2-Methylnaphthalene

2-Naphthylamine

2-Nitrophenol

2-sec-Butyl-4,6-dinitrophenol

3,3"-Dichlorobenzidine

3,3"-Dimethylbenzidine

3-Methylcholanthrene

4-Aminobiphenyl

4-Bromophenyl phenyl ether

4-Chloro-m-cresol

\author{
Screen Zone Elevation \\ $162.7 \cdot 141.7 \mathrm{ft} \mathrm{msl}$ \\ 03/07/00
}

1st Half
163.12
5.3
17
19.1
1
.7
3.74

1st Half
$<5$
$<5$
$<5$
$<5$
$<5$
$<5$
$<5$
$<5$

\begin{tabular}{cc} 
CLP EPA & Fill. \\
\hline$U$ & $<E Q L$ \\
$U$ & $<E Q L$ \\
$U$ & $<E Q L$ \\
$U$ & $<E Q L$ \\
$U$ & $<E Q L$ \\
$U$ & $<E Q L$ \\
$U$ & $<E Q L$ \\
$U$ & $<E Q L$
\end{tabular}

$<10 \quad U<E Q L$

$<5 \quad U \quad<$ EQL

$<5 \quad U<$ EQL

$<5$

$<5$

$<5 \quad$ v

$<5$

$<5$

$<E Q L$

$<E Q L$

$<5$

u

$<E Q L$

$<E Q L$

$<E Q L$

$<$ EQL
Casing

4 " PVC

$\frac{\text { Pump }}{\mathrm{S}}$

Screen Zone

Upper

$08 / 17 / 00$

$\begin{array}{ll}\text { 2nd Half } & \text { Unit } \\ 162.4 & \text { ft msl } \\ 5.6 & \mathrm{pH} \\ 17 & \text { uS/cm } \\ 23.9 & \text { deg. C } \\ 1 & \mathrm{mg} / \mathrm{L} \\ .6 & \text { NTU } \\ 3.87 & \text { well volume }\end{array}$

Notes: Concentrations in bold italics exceed the groundwater protection standards listed in Appendix A. Bold italics were not assigned if the result was qualified with a "J", "R", "L", or "U" qualifier.

$+=$ exceeded the Groundwater Protection Standards listed in Appendix A. 
Table B. Assessment Monitoring Results for Groundwater Wells (Cont.)

\section{WELL LFW 30}

\section{ANALYTICAL DATA}

Groundwater Protection Standard

\section{ST Parameter}

$\underline{\text { Unit }}$

4-Chloroaniline

4-Chlorophenyl phenyl ether

4-Nitrophenol

5-Nitro-o-toluidine

7,12-Dimethylbenz(a)anthracene

Acenaphthene

Acenaphthylene

Acetone

Acetonitrile (Methyl cyanide)

Acetophenone

Acrolein

Acrylonitrile

Aldrin

Allyl chloride

Anthracene

Antimony, total recoverable

Arsenic, total recoverable

Barium, total recoverable

Benzene

Benzo(a)anthracene

Benzo(a)pyrene

Benzo(b)fluoranthene

Benzo(g,h,i)perylene

Benzo(k)fluoranthene

Benzyl alcohol

Beryllium, total recoverable

Bis(2-chloroethoxy) methane

Bis(2-chloroethyl) ether

Bis(2-ethylhexyl) phthalate

Bromochloromethane

Bromodichloromethane

Bromoform

Bromomethane (Methyl bromide)

Butylbenzyl phthalate

Cadmium, total recoverable

Carbon disulfide

Carbon tetrachloride

Chlorobenzene

Chlorobenzilate

Chloroethane

Chloroethene (Vinyl chloride)

Chloroform

Chloromethane (Methyl chioride)

Chloroprene

Chromium, total recoverable

Chrysene

Cobalt, total recoverable

Copper, total recoverable

Cyanide

Di-n-butyl phthalate

Di-n-octyl phthalate

Diallate

Dibenz(a,h)anthracene

Dibenzofuran

Dibromochloromethane

Dibromomethane (Methylene bromide)

Dichlorodifluoromethane

Dichloromethane (Methylene chloride)

Dieldrin

Diethyl phthalate

Dimethoate

Dimethyl phthalate

Diphenylamine

Disulfoton

Endosulfan 1

Endosulfan II

Endosulfan sulfate

Endrin

$\begin{array}{lllllll}<20 & \mathrm{U} & <\mathrm{EQL} & <20 & \mathrm{U} & <\mathrm{EQL} & \mathrm{ug} / \mathrm{L} \\ <200 & \mathrm{U} & <\mathrm{EQL} & <200 & \mathrm{U} & <\mathrm{EQL} & \mathrm{ug} / \mathrm{L} \\ <50 & \mathrm{U} & <\mathrm{EQL} & <50 & \mathrm{U} & <\mathrm{EQL} & \mathrm{ug} / \mathrm{L} \\ <10 & \mathrm{U} & <\mathrm{EQL} & <10 & \mathrm{U} & <\mathrm{EQL} & \mathrm{ug} / \mathrm{L} \\ & & & & & & \\ <5 & \mathrm{U} & <\mathrm{EQL} & <5 & \mathrm{EQL} & \mathrm{ug} / \mathrm{L} \\ & & & & & & \\ <100 & \mathrm{U} & <\mathrm{EQL} & <100 & \mathrm{E} & <\mathrm{EQL} & \mathrm{ug} / \mathrm{L} \\ <10 & \mathrm{U} & <\mathrm{EQL} & <10 & \mathrm{U} & <\mathrm{EQL} & \mathrm{ug} / \mathrm{L} \\ <5.48 & \mathrm{U} & \mathrm{rEQL} & 5.46 & \mathrm{~J} & \mathrm{NDD} & \mathrm{ug} / \mathrm{L} \\ <5 & \mathrm{U} & <\mathrm{EQL} & <5 & \mathrm{U} & <\mathrm{EQL} & \mathrm{ug} / \mathrm{L}\end{array}$

$<1 \quad$ U 1 EQL $\quad<1 \quad U \quad<E Q L \quad u g / L$

$<5 \quad U<E Q$

$<$ EQL

$<$ EQL

$<$ EQL

$\begin{array}{lll}<10 & U & <E Q L \\ <5 & U & <E Q L\end{array}$

$<\mathrm{EQL}$

$<E Q L$

$<E Q L$

$<E Q L$

$<$ EQL

$<$ EQL

$<E Q L$

EQL

$<3.83 \mathrm{JU}<\mathrm{EQL}$

$<20 \quad U \quad<E Q L$

13.2 J I NDD

$<11$ JU $\mathrm{L}<\mathrm{EQL}$

$<5$
$<5$
$<5$
$<5$

U

(1)

$\mathrm{ug} / \mathrm{L}$

$<10 \quad U<E Q L$

$<5 \quad U<E Q$

$<E Q L$

$<E Q L$

$<E O L$

$<E Q L$

$\mathrm{ug} / \mathrm{L}$

ug/L

$u g / L$

ug/L

$\mathrm{ug} / \mathrm{L}$

$\mathrm{ug} / \mathrm{L}$

ug/L

$\mathrm{ug} / \mathrm{L}$

$<E Q L$

$<$ EQL

$\mathrm{ug} / \mathrm{L}$

$<E Q L$

$<E Q L$

$<$ EQL

$<10 \quad \mathrm{E} \quad<\mathrm{EQL} \quad \mathrm{ug} / \mathrm{L}$

ug/L

$\mathrm{ug} / \mathrm{L}$

ug/L

$\mathrm{ug} / \mathrm{L}$

$<20 \quad \mathrm{E} \quad<\mathrm{EQL} \quad \mathrm{ug} / \mathrm{L}$

$<17.4 \quad U \quad V \quad<E Q L \quad u g / L$

$<10$ JU $Q \quad<E Q L \quad u g / L$

Notes: Concentrations in bold italics exceed the groundwater protection standards listed in Appendix A. Bold italics were not assigned if the result was quatified with a "J", "R", "L", or "U" qualifier.

$+=$ exceeded the Groundwater Protection Standards listed in Appendix A. 


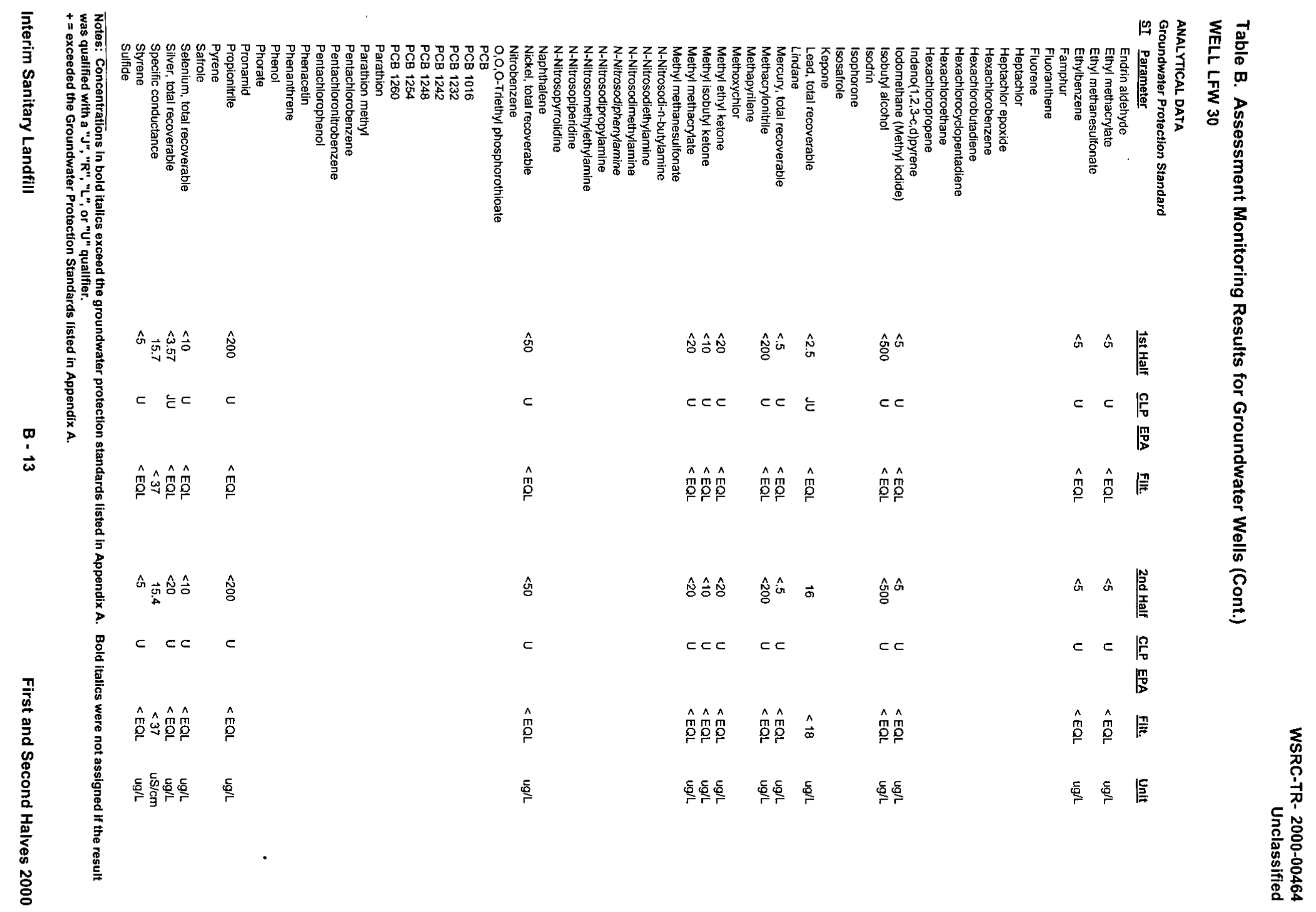


Table B. Assessment Monitoring Results for Groundwater Wells (Cont.)

WELL LFW 30

ANALYTICAL DATA

Groundwater Protection Standard

ST Parameter

Tetrachloroethylene

Thallium, total recoverabie

Thionazin

Tin, total recoverable

Toluene

Toxaphene

Trichloroethylene

Trichlorofluoromethane

Vanadium, total recoverable

Vinyl acetate

Xylenes

Zinc, total recoverable

alpha-Benzene hexachloride

alpha-Chlordane

beta-Benzene hexachloride

cis-1,2-Dichloroethylene

cis-1,3-Dichloropropene

delta-Benzene hexachloride

gamma-Chlordane

m-Cresol (3-Methylphenol)

$\mathrm{m}$-Nitroaniline

o-Cresol (2-Methytphenal)

o-Nitroaniline

o-Toluidine

p.p"-DDD

$p, p^{\prime \prime-D D E ~}$

p.p"-DDT

p-Cresol (4-Methylphenol)

p-Dimethylaminoazobenzene

p-Nitroaniline

p-Phenylenediamine

$\mathrm{pH}$

trans-1,2-Dichloroethylene

trans-1,3-Dichloropropene

trans-1,4-Dichloro-2-butene

\begin{tabular}{|c|c|c|c|}
\hline st Half & CLP & EPA & Fil \\
\hline$<5$ & U & & $<\mathrm{EC}$ \\
\hline$<6.28$ & JU & V & $<E C$ \\
\hline$<5$ & $U$ & & $<\mathrm{EC}$ \\
\hline$<5$ & $U$ & & $<\mathrm{EC}$ \\
\hline 2.4 & \rfloor & I & ND \\
\hline$<10$ & $U$ & & $<E O$ \\
\hline$<5$ & $U$ & & $<E C$ \\
\hline$<10$ & U & & $<E O$ \\
\hline 18.4 & $\mathrm{~J}$ & 1 & $\mathrm{NL}$ \\
\hline$<.1$ & U & & $<$ \\
\hline$<5$ & U & & 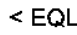 \\
\hline$<5$ & U & & \\
\hline
\end{tabular}

\begin{tabular}{|c|c|c|}
\hline 2nd Half & CLP EPA & Filt, \\
\hline$<5$ & $U$ & $<$ EQL \\
\hline$<10$ & $U$ & $<E Q L$ \\
\hline$<5$ & U & $<E Q L$ \\
\hline$<5$ & U & $<E Q L$ \\
\hline$<5$ & U & $<E Q L$ \\
\hline$<10$ & u & $<\mathrm{EQL}$ \\
\hline$<5$ & u & $<E Q L$ \\
\hline$<10$ & u & $<\mathrm{EQL}$ \\
\hline 24.6 & & $<29.3$ \\
\hline
\end{tabular}

$\begin{array}{lllll}<.095 & \text { JU } & Q & <E Q L & \text { ug/L } \\ <5 & U & & <E Q L & \text { ug/L }\end{array}$

$<5 \quad U<\mathrm{EQL} \quad \mathrm{ug} / \mathrm{h}$

$\begin{array}{llll}\quad 5.64 & \mathrm{~J} & \mathrm{Q} & \mathrm{NDD} \\ <5 & U & & <\mathrm{EQL} \\ <5 & U & & <\mathrm{EQL} \\ <20 & U & & <\mathrm{EQL}\end{array}$

$\begin{array}{lllll}5.18 & \mathrm{~J} & \mathrm{Q} & \mathrm{NDD} & \mathrm{pH} \\ <5 & \mathrm{U} & & <\mathrm{EQL} & \mathrm{ug} / \mathrm{L} \\ <5 & U & & <\mathrm{EQL} & \mathrm{ug} / \mathrm{L} \\ <20 & U & & <\mathrm{EQL} & \mathrm{ug} / \mathrm{L}\end{array}$

Notes: Concentrations in bold italics exceed the groundwater protection standards listed in Appendix A. Bold italics were not assigned if the result was qualified with a "J", "R", "L", or "U" qualifier.

$+=$ exceeded the Groundwater Protection Standards listed in Appendix A. 
Table B. Assessment Monitoring Results for Groundwater Wells (Cont.)

WELL LFW 31

$\begin{array}{ll}\text { SRS Coord. } & \text { Lat/Longitude } \\ \text { N } 86262.2 & \text { 33.28976 Deg N } \\ \text { E 44869 } & -81.7162 \text { Deg W }\end{array}$

SAMPLE DATE

\section{FIELD DATA}

\section{Parameter}

Water Elevation
pH
Sp. Conductance
Water temperature
Alkalinity as $\mathrm{CaCO} 3$
Turbidity
Volumes purged
Sampling code

ANALYTICAL DATA

Groundwater Protection Standard ST Parameter

1,1,1,2-Tetrachloroethane

1,1,1-Trichloroethane

1,1,2,2-Tetrachloroethane

1,1,2-Trichloroethane

1,1-Dichloroethane

1,1-Dichloroethylene

1,1-Dichloropropene

1,2,3-Trichloropropane

1,2,4,5-Tetrachlorobenzene

1,2,4-Trichlorobenzene

1,2-Dibromo-3-chloropropane

1,2-Dibromoethane

1,2-Dichlorobenzene

1,2-Dichloroethane

1,2-Dichloropropane

1,3,5-Trinitrobenzene

1,3-Dichlorobenzene

1,3-Dichloropropane

1,3-Dinitrobenzene

1,4-Dichlorobenzene

1,4-Naphthoquinone

1-Naphthylamine

2,2-Dichloropropane

2,2-Oxybis(1-chloropropane)

2,3,4,6-Tetrachlorophenol

2,4,5-T

2,4,5-TP (Silvex)

2,4,5-Trichlorophenol

2,4,6-Trichlorophenol

2,4-Dichlorophenol

2,4-Dichlorophenoxyacetic acid

2,4-Dimethyl phenol

2,4-Dinitrophenol

2,4-Dinitrotoluene

2,6-Dichlorophenol

2,6-Dinitrotoluene

2-Acetylaminofluorene

2-Chloronaphthalene

2-Chlorophenot

2-Hexanone

2-Methyl-4,6-dinitropheno

2-Methylnaphthaiene

2-Naphthylamine

2-Nitrophenol

2-sec-Butyl-4,6-dinitrophenol

3,3 "-Dichlorobenzidine

3,3"-Dimethylbenzidine

3-Methylcholanthrene

4-Aminobiphenyl

4-Bromophenyl phenyl ether

4-Chloro-m-cresol

\author{
Screen Zone Elevation \\ $166-145 \mathrm{ft} \mathrm{mst}$
}

03/07/00

\begin{tabular}{l} 
1st Half \\
\hline 162.94 \\
5.2 \\
15 \\
18.5 \\
2 \\
1.7 \\
4.15
\end{tabular}

1st Half CLP EPA

$<5$
$<5$
$<5$
$<5$
$<5$
$<5$
$<5$
$<5$

Filt.

$<E Q L$

$<E Q L$

$<E Q L$

$<E Q L$

$<$ EQL

$<$ EQL

$<E Q L$

$<10 \quad U<E Q$

$<10 \quad U<E Q$

$\begin{array}{lll}<5 & U & <E Q L \\ <5 & U & <E Q L\end{array}$

$<5 \quad U<E Q L$

$<5 \quad U<E Q L$

$<5 \quad U \quad<E Q$

$<5 \quad U \quad<$ EQL

$<5 \quad U \quad<E Q L$

$<5 \quad U<E Q L$

$<20$

u

$<E Q L$

$<20$

u

$<E Q L$

$\mathrm{ug} / \mathrm{L}$

Notes: Concentrations in bold italics exceed the groundwater protection standards listed in Appendix A. Bold italics were not assigned if the result was qualified with a "J", "R", "L", or "U" qualifier.

$+=$ exceeded the Groundwater Protection Standards listed in Appendix A. 
Table B. Assessment Monitoring Results for Groundwater Wells (Cont.)

WELL LFW 31

ANALYTICAL DATA

Groundwater Protection Standard

ST Parameter

1st Half CLP EPA Filt.

2nd Half CLP EPA Filt. Unit

4-Chloroaniline

4-Chlorophenyl phenyl ether

4-Nitrophenol

5-Nitro-o-toluidine

7,12-Dimethylbenz(a)anthracene

Acenaphthene

Acenaphthylene

Acetone

Acetonitrile (Methyl cyanide)

Acetophenone

Acrolein

Acrylonitrile

Aldrin

Allyl chloride

Anthracene

Antimony, total recoverable

Arsenic, total recoverable

Barium, total recoverable

Benzene

Benzo(a)anthracene

Benzo(a)pyrene

Benzo(b)fluoranthene

Benzo(g,h,i)perylene

Benzo(k)fluoranthene

Benzyl alcohol

Beryllium, total recoverable

Bis(2-chloroethoxy) methane

Bis(2-chloroethyl) ether

Bis(2-ethylhexyl) phthalate

Bromochloromethane

Bromodichloromethane

Bromoform

Bromomethane (Methyl bromide)

Butylbenzyl phthalate

Cadmium, total recoverable

Carbon disulfide

Carbon tetrachloride

Chlorobenzene

Chlorobenzilate

Chloroethane

Chloroethene (Vinyl chloride)

Chloroform

Chloromethane (Methyl chloride)

Chloroprene

Chromium, total recoverable

Chrysene

Cobalt, total recoverable

+ Copper, total recoverable

Cyanide

Di-n-butyl phthatate

Di-n-octyl phthalate

Diallate

Dibenz(a,h)anthracene

Dibenzofuran

Dibromochloromethane

Dibromomethane (Methylene bromide)

Dichlorodifluoromethane

Dichloromethane (Methylene chloride)

Dieldrin

Diethyl phthalate

Dimethoate

Dimethyl phthalate

Diphenylamine

Disulfoton

Endosulfan I

Endosulfan II

Endosulfan sulfate

Endrin

$\begin{array}{lll}<20 & U & <E Q L \\ <200 & U & <E Q L \\ & & \\ <50 & U & <E Q L \\ <10 & U & <E Q L \\ & & \\ <5 & U & <E Q L \\ & & \\ <100 & U & <E Q L \\ <10 & U & <E Q L \\ 4.29 & J & \quad \\ <5 & U & <E D L\end{array}$

U

$<\mathrm{EQL}$

$<1$

u

$<E Q L$

ug/L

$<5 \quad U<\mathrm{EQL}$

$<5 \quad U<E Q L$

$<5 \quad \mathrm{U}<\mathrm{EQL}$

$<5 \quad U<$ EQL

$<10 \quad U \quad<$ EQL

$<5 \quad U<E Q L$

$<5 \quad U$

$<5 \quad U<E Q$

$<5 \quad U \quad<E Q L$

$<5 \quad U \quad<E Q L$

$<5 \quad U \quad<E Q L$

$<5 \quad U \quad<E Q L$

$<20 \quad U<<E Q L$

$<10 \quad U<$

$<20 \quad U<$ EQL

$6.78 \mathrm{~J} \quad \mathrm{~N}$ NDD

$<10$ JU $\mathrm{L}<\mathrm{EQL}$

$<5$
$<5$
$<5$
$<5$
$<10$
$<5$
$<5$
$<5$

$<5$

$<5$

$<5$

$<5$

$<20$

$<10$

$<20$

40.8

$<9.5$

JU

$U<E Q L$

$<E Q L$

$<E Q L$

$<E Q L$

ug/L

ug/L

ug/L

$<E Q L$

$<E Q L$

$<E Q L$

$<$ EQL

ug/L

ug/L

ug/L

$\mathrm{ug} / \mathrm{L}$

$<$ EQL

$<E Q L$

$<E Q L$

$<E Q L$

$<E Q L$

$<\mathrm{EQL}$

$<$ EQL

$>29.8$

ug/L

ug/L

ug/L

ug/L

$\mathrm{ug} / \mathrm{L}$

ug/L

ug/L

ug/L

ug/L

$\begin{array}{llll}<5 & U & & <E Q L \\ <5 & U & & <E Q L \\ <5 & U & & <E Q L \\ <2.5 & U & V & <E Q L\end{array}$

ug/L

ug/

$u g / L$

$\mathrm{ug} / \mathrm{L}$

Notes: Concentrations in bold italics exceed the groundwater protection standards listed in Appendix A. Bold italics were not assigned if the result was qualified with a "J", "R", "L", or "U" qualifier.

$+=$ exceeded the Groundwater Protection Standards listed in Appendix A. 
Table B. Assessment Monitoring Results for Groundwater Wells (Cont.)

WELL LFW 31

ANALYTICAL DATA

Groundwater Protection Standard

\section{ST Parameter}

Endrin aldehyde

Ethyl methacrylate

Ethyl methanesulfonate

Ethylbenzene

Famphur

Fluoranthene

Fluorene

Heptachlor

Heptachlor epoxide

Hexachlorobenzene

Hexachlorobutadiene

Hexachlorocyclopentadiene

Hexachloroethane

Hexachloropropene

Indeno(1,2,3-c,d)pyrene

lodornethane (Methyl iodide)

Isobutyl alcohol

Isodrin

Isophorone

Isosafrole

Kepone

Lead, total recoverable

Lindane

Mercury, total recoverable

Methacrylonitrile

Methapyrilene

Methoxychlor

Methyl ethyl ketone

Methyl isobutyl ketone

Methyl methacrylate

Methyl methanesulfonate

$N$-Nitrosodi-n-butylamine

$\mathrm{N}$-Nitrosodiethylamine

$\mathrm{N}$-Nitrosodimethylamine

$\mathrm{N}$-Nitrosodiphenylamine

$\mathrm{N}$-Nitrosodipropylamine

$\mathrm{N}$-Nitrosomethylethylamine

N-Nitrosopiperidine

$\mathrm{N}$-Nitrosopyrrolidine

Naphthalene

Nickel, total recoverable

Nitrobenzene

O,0,0-Triethyl phosphorothioate

PCB

PCB 1016

PCB 1232

PCB 1242

PCB 1248

PCB 1254

PCB 1260

Parathion

Parathion methy

Pentachlorobenzene

Pentachloronitrobenzene

Pentachlorophenol

Phenacetin

Phenanthrene

Phenol

Phorate

Pronamid

Propionitrile

Pyrene

Safrole

Selenium, total recoverable

Silver, total recoverable

Specific conductance

Styrene

Sulfide

$\begin{array}{lll}\text { 1st Half } & \text { CLP EPA } & \text { Filt. } \\ <5 & U & <E Q L \\ <5 & U & <E Q L\end{array}$

2ndHalf CLP EPA Filt.

Unit

$\begin{array}{llll}<5 & U & <E Q L & u g / L\end{array}$

$<5$

$<\mathrm{EQL}$

$<5$

$<500$

$<$ EQL

$u g / L$

$<500 \quad U$

U

$<E Q L$

$u g / L$

$<3.05$ JU $\quad<E Q L$

$<10 \quad U<E Q L$

$\mathrm{ug} / \mathrm{L}$

$<.5 \quad U \quad<E Q L$

$<200$

$<E Q L$

$<.5$

$<200$

$<\mathrm{EQL}$

$u g / L$

$u g / L$

$<20 \quad U<E Q$

$<10$

$<20$

$<$ EQL

$<\mathrm{EQL}$

$<20$
$<10$

$<10$
$<20$

$<E Q L$

$<$ EQL

$<$ EQL

$u g / L$

ug/h

ug/L

$c$

$<50$

U

< EQL

$<50$

U

$<\mathrm{EQL}$

ug/L

$\begin{array}{ccccccc}<200 & \mathrm{U} & <\mathrm{EQL} & <200 & \mathrm{U} & <\mathrm{EQL} & \mathrm{ug} / \mathrm{L} \\ & & & & & \\ 12.3 & <50 & <10 & \mathrm{U} & <\mathrm{EQL} & \mathrm{ug} / \mathrm{L} \\ 46.1 & <100 & <20 & \mathrm{U} & <\mathrm{EQL} & \mathrm{ug} / \mathrm{L} \\ 14.8 & & 18.9 & & <37 & \mathrm{uS} / \mathrm{cm} \\ <5 & \mathrm{U} & <\mathrm{EQL} & <5 & \mathrm{U} & <\mathrm{EQL} & \mathrm{ug} / \mathrm{L}\end{array}$

Notes: Concentrations in bold italics exceed the groundwater protection standards listed in Appendix A. Bold italics were not assigned if the result was qualified with a "J", "R", "L", or "U" qualifier.

$+=$ exceeded the Groundwater Protection Standards listed in Appendix A. 
Table B. Assessment Monitoring Results for Groundwater Wells (Cont.)

\section{WELL LFW 31}

ANALYTICAL DATA

Groundwater Protection Standard

ST Parameter

Tetrachloroethylene

Thallium, total recoverable

Thionazin

Tin, total recoverable

Toluene

Toxaphene

Trichloroethylene

Trichlorofluoromethane

Vanadium, total recoverable

Vinyl acetate

Xylenes

+ Zinc, total recoverable

alpha-Benzene hexachloride alpha-Chlordane

beta-Benzene hexachloride

cis-1,2-Dichloroethylene

cis-1,3-Dichloropropene

delta-Benzene hexachloride

gamma-Chlordane

m-Cresol (3-Methylphenol)

m-Nitroaniline

o-Cresol (2-Methylphenol)

o-Nitroaniline

$o$-Toluidine

p.p"-DDD

$p, p^{\prime \prime}-D D E$

$p, p$ "-DDT

p-Cresol (4-Methylphenol)

p-Dimethylaminoazobenzene

p-Nitroaniline

p-Phenylenediamine

$\mathrm{pH}$

trans-1,2-Dichloroethylene

trans-1,3-Dichloropropene

trans-1,4-Dichloro-2-butene

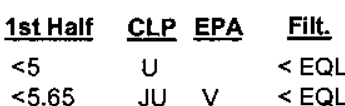

$<5.65$ JU $V<E Q L$

$<5 \quad U \quad<E Q L$

$<5 \quad U<E Q$

1.5 J IL NDD

$<10 \quad U \quad<$ EQL

$<5 \quad U<E Q$

$<10 \quad U<E Q L$

11 J I NDD

$\begin{array}{lll}<.1 & U & <E Q L \\ <5 & U & <E Q L \\ <5 & U & <E Q L\end{array}$

$\begin{array}{llll}\quad 5.73 & \mathrm{~J} & \mathrm{Q} & \mathrm{NDD} \\ <5 & \mathrm{U} & & <\mathrm{EQL} \\ <5 & \mathrm{U} & & <\mathrm{EQL} \\ <20 & \mathrm{U} & & <E Q L\end{array}$

. $<20 \quad U \quad<E Q L$

$\begin{array}{cccc}\text { 2nd Half } & \text { CLP EPA } & \begin{array}{c}\text { Filt. } \\ <5\end{array} & \text { Unit } \\ <10 & U & <E Q L & \mathrm{ug} / \mathrm{L} \\ \mathrm{U} & <E Q L & \mathrm{ug} / \mathrm{L}\end{array}$

$<5$

3.7

$<10$

$<5$

$<10$

40.2

U

$<E Q L$

$\operatorname{ug} / \mathrm{L}$

ug/

ug/L

$u g / L$

$\mathrm{ug} / \mathrm{L}$

$\mathrm{ug} / \mathrm{L}$

$\mathrm{ug} / \mathrm{L}$

$\begin{array}{lllll}<.095 & \mathrm{JU} & \mathrm{Q} & <\mathrm{EQL} & \mathrm{ug} / \mathrm{L} \\ <5 & \mathrm{U} & & <\mathrm{EQL} & \mathrm{ug} / \mathrm{L} \\ <5 & \mathrm{U} & & <\mathrm{EQL} & \mathrm{ug} / \mathrm{L}\end{array}$

$u g / L$
$u g / L$
$u g / L$

$\begin{array}{lllll}5.24 & \mathrm{~J} & \mathrm{Q} & \mathrm{NDD} & \mathrm{pH} \\ <5 & \mathrm{U} & & <\mathrm{EQL} & \mathrm{ug} / \mathrm{L} \\ <5 & \mathrm{U} & & <\mathrm{EQL} & \mathrm{ug} / \mathrm{h} \\ <20 & \mathrm{U} & & <\mathrm{EQL} & \mathrm{ug} / \mathrm{L}\end{array}$

Notes: Concentrations in bold italics exceed the groundwater protection standards listed in Appendix A. Bold italics were not assigned if the result was qualified with a "J", "R", "L", or "U" qualifier.

$+=$ exceeded the Groundwater Protection Standards listed in Appendix A. 
Table B. Assessment Monitoring Results for Groundwater Wells (Cont.)

WELL LFW 32

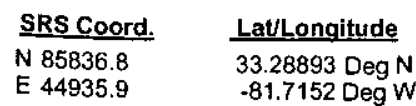

SAMPLE DATE FIELD DATA

\section{Parameter}

Water Elevation

$\mathrm{pH}$

Sp. Conductance

Water temperature

Alkalinity as $\mathrm{CaCO}$

Turbidity

Volumes purged

Sampling code

\section{ANALYTICAL DATA}

Groundwater Protection Standard

ST Parameter

1,1,1,2-Tetrachloroethane

1,1,1-Trichloroethane

1,1,2,2-Tetrachloroethane

1,1,2-Trichloroethane

1,1-Dichloroethane

1,1-Dichloroethylene

1,1-Dichloropropene

1,2,3-Trichloropropane

1,2,4,5-Tetrachlorobenzene

1,2,4-Trichlorobenzene

1,2-Dibromo-3-chloropropane

1,2-Dibromoethane

1,2-Dichlorobenzene

1,2-Dichloroethane

1,2-Dichloropropane

1,3,5-Trinitrobenzene

1,3-Dichlorobenzene

1,3-Dichloropropane

1,3-Dinitrobenzene

1,4-Dichlorobenzene

1,4-Naphthoquinone

1-Naphthylamine

2,2-Dichloropropane

2,2-Oxybis(1-chloropropane)

2,3,4,6-Tetrachloropheno

2,4,5-T

2,4,5-TP (Silvex)

2,4,5-Trichlorophenol

2,4,6-Trichlorophenol

2,4-Dichlorophenol

2,4-Dichlorophenoxyacetic acid

2,4-Dimethyl phenol

2,4-Dinitrophenol

2,4-Dinitrotoluene

2,6-Dichlorophenol

2,6-Dinitrotoluene

2-Acetylaminofluorene

2-Chjoronaphthalene

2-Chlorophenol

2-Hexanone

2-Methyl-4,6-dinitrophenol

2-Methyinaphthalene

2-Naphthylamine

2-Nitrophenol

2-sec-Butyl-4,6-dinitrophenol

3,3"-Dichlorobenzidine

3,3"-Dimethylbenzidine

3-Methylcholanthrene

4-Aminobiphenyl

4-Bromophenyl phenyl ether

4-Chloro-m-cresol

\section{Screen Zone Elevation \\ $165.3-144.3 \mathrm{ft}$ mst}

$03 / 08 / 00$

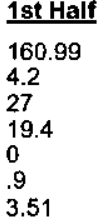

1st Half

$<5$

4.6 J $\quad$ I $\quad$ NDD

$<5 \quad U \quad<E Q L$

$<5 \quad U<E Q$

2.5 J I NDD

$<5 \quad U<E Q L$

$<5 \quad U<E Q$

$<5 \quad U<E Q$

$<53$ JU $Q<E Q L$

$<11$ JU Q $Q E Q L$

$<10 \quad U<E Q L$

$<5 \quad U<E Q L$

$<5 \quad U<E Q$

$<E Q L$

$<$ EQL

$<E Q L$

$<\mathrm{EQL}$

$<E Q L$

$<E Q L$

$<E Q L$

$<$ EQL

$<E Q L$

$<\mathrm{EQL}$

$<$ EQL

$<$ EQL

$<$ EQL

$<\mathrm{EQL}$

$<E Q L$

$<$ EQL

$<$ EQL

$<E Q L$

$<E Q L$

$<$ EQL

< EQL

$<$ EQL

$<$ EQL

$<$ EQL

$<\mathrm{EQL}$

$<E Q L$

$<E Q L$

$<E Q L$

$<$ EQL

$<$ EQL

$<E Q L$

$<\mathrm{EQL}$

$<\mathrm{EQL}$

$<E Q L$

$<$ EQL

$<$ EQL

$<E Q L$
Unit

$\mathrm{ft}$ msl

$\mathrm{pH}$

US $/ \mathrm{cm}$

deg. C

mg/L

well volume een Zone

Upper

$\begin{array}{ll}160.45 & \mathrm{ft} \mathrm{msl} \\ 4.8 & \mathrm{pH} \\ 29 & \text { uS/cm } \\ 20.7 & \mathrm{deg} . \mathrm{C} \\ 0 & \mathrm{mg} / \mathrm{L} \\ .6 & \mathrm{NTU} \\ 3.25 & \text { well volume }\end{array}$

Notes: Concentrations in boid italics exceed the groundwater protection standards listed in Appendix A. Bold italics were not assigned if the result was qualified with a "J", "R", "L", or "U" qualifier.

$+=$ exceeded the Groundwater Protection Standards listed in Appendix A. 


\section{ANALYTICAL DATA}

Groundwater Protection Standard

ST Parameter

4-Chloroaniline

4-Chlorophenyl phenyl ether

4-Nitrophenol

5-Nitro-o-toluidine

7,12-Dimethylbenz(a)anthracene

Acenaphthene

Acenaphthylene

Acetone

Acetonitrile (Methyl cyanide)

Acetophenone

Acrolein

Acrylonitrile

Aldrin

Allyl chloride

Anthracene

Antimony, total recoverable

Arsenic, total recoverable

Barium, total recoverable

Benzene

Benzo(a)anthracene

Benzo(a)pyrene

Benzo(b)fluoranthene

Benzo(g,h,i)perylene

Benzo(k)fluoranthene

Benzyl alcohol

Beryllium, total recoverable

Bis(2-chloroethoxy) methane

Bis (2-chioroethyl) ether

Bis(2-ethylhexyl) phthalate

Bromochloromethane

Bromodichloromethane

Bromoform

Bromomethane (Methyl bromide)

Butylbenzyl phthalate

Cadmium, total recoverable

Carbon disulfide

Carbon tetrachloride

Chlorobenzene

Chlorobenzilate

Chloroethane

Chloroethene (Vinyl chloride)

Chloroform

Chloromethane (Methyi chloride)

Chloroprene

Chromium, total recoverable

Chrysene

Cobalt, total recoverable

Copper, total recoverable

Cyanide

Di-n-butyl phthalate

Di-n-octyl phthalate

Diallate

Dibenz(a,h)anthracene

Dibenzofuran

Dibromochloromethane

Dibromomethane (Methylene bromide)

Dichlorodifluoromethane

Dichloromethane (Methylene chloride)

Dieldrin

Diethyl phthalate

Dimethoate

Dimethyl phthalate

Diphenylamine

Disulfoton

Endosulfan I

Endosulfan II

Endosulfan sulfate

Endrin

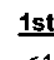

$<11$ JU $\mathrm{Q}<\mathrm{EQL}$

$<11$ JU $Q<E Q L$

$<26$ JU $Q \quad<E Q L$

$<11$ JU Q $<E Q L$

$<11$ JU $Q<E Q L$

$<11$ JU $Q<E Q L$

$<11$ JU $Q<E Q L$

$<20 \quad U<E Q$

$<200 \quad U<E Q$

$<11$ JU $Q<E Q$

$<50 \quad U \quad<E Q L$

$<10 \quad U<\angle O L$

$<1 \quad U \quad<E Q L$

$<5 \quad U<E Q L$

$<11$ JU $Q<E Q$

$<100 \quad \mathrm{E}<\mathrm{EQL}$

$<10 \quad U<$ EQL

$\begin{array}{lll} & 10.9 & <2000 \\ <5 & U & <E Q L\end{array}$

$<11$ JU $Q<E Q L$

$<11$ JU $Q \quad<E Q L$

$<11$ JU $Q<E Q L$

$<11$ JU $Q<E Q$

$<11$ JU $Q<E Q$

$<1 \quad U \quad<E Q L$

$<11$ JU $Q<E Q L$

$<11$ JU $Q<E Q L$

$<11$ JU $Q<E Q L$

$<5 \quad U<E Q$

$<5 \quad U<E Q$

$<5 \quad U \quad<E Q$

$<5 \quad U<$ EQL

$<11$ JU $Q<E Q L$

$<10 \quad U<E Q$

$<5 \quad U<E Q$

$<E Q L$

$<E Q L$

$Q<E Q L$

$<\mathrm{EQL}$

$<E Q L$

$<E Q L$

$<E Q L$

$<E Q L$

$<E Q L$

$<$ EQL

$<E Q L$

NDD

Rejected

$<$ EQL

$<$ EQL

$<E Q L$

$<E Q L$

$<\mathrm{EQL}$

$<$ EQL

$<E Q L$

$<E Q L$

NDD

$<E Q L$

$<E Q L$

$<E Q L$

$<$ EQL

$<E Q L$

$<E Q L$

$<$ EQL

$<E Q L$

$<\mathrm{EQL}$

$<E Q L$ 2nd Haif CLP EPA Filt. Unit

$\mathrm{ug} / \mathrm{L}$

$\mathrm{ug} / \mathrm{L}$

$\mathrm{ug} / \mathrm{L}$

ug/L

$\mathrm{ug} / \mathrm{L}$

$\mathrm{ug} / \mathrm{L}$

$<20 \quad U \quad<E Q L \quad u g / L$

$<200 \quad \mathrm{U} \quad<\mathrm{EQL} \quad \mathrm{ug} / \mathrm{L}$

$<50 \quad \mathrm{U}<\mathrm{EQL} \quad \mathrm{ug} / \mathrm{L}$

$<10 \quad \mathrm{E} \quad<\mathrm{EQL} \mathrm{ug} / \mathrm{h}$

$<5 \quad U \quad<E Q L \quad \mathrm{ug} / \mathrm{L}$

ug/L

$<100 \quad \mathrm{U} \quad<\mathrm{EQL} \quad \mathrm{ug} / \mathrm{L}$

$<4.21$ JU $<$ EQL Ug/L

$11.4<2000 \quad$ ug/L

$<5 \quad \mathrm{U}<\mathrm{EQL} \quad \mathrm{ug} / \mathrm{L}$

$u g / L$

ug/L

$u g / L$

$u g /$

$u g / L$

$<19 \quad$ JU $\quad<E Q L \quad u g / L$

$u g / L$
$\omega g / L$

$\mathrm{ug} / \mathrm{L}$

$\begin{array}{llll}<5 & \mathrm{U} & <\mathrm{EQL} & \mathrm{ug} / \mathrm{L}\end{array}$

$<5 \quad U \quad<E Q L \quad u g / L$

$<5 \quad \mathrm{E}<\mathrm{EQL} \quad \mathrm{ug} / \mathrm{L}$

$\mathrm{ug} / \mathrm{L}$

$<5 \quad \mathrm{U} \quad<\mathrm{EQL} \quad \mathrm{ug} / \mathrm{L}$

$<5 \quad U$

$<5 \quad \mathrm{U} \quad<\mathrm{EQL} \quad \mathrm{ug} / \mathrm{L}$

ug/L

$<5 \quad U \quad<E Q L \quad u g / L$

$<5 \quad U<E Q L \quad \mathrm{ug} / \mathrm{L}$

$<5 \quad \mathrm{U}<\mathrm{EQL} \quad \mathrm{ug} / \mathrm{L}$

$<5 \quad \mathrm{U}<\mathrm{EQL} \quad \mathrm{ug} / \mathrm{L}$

$<20 \quad \mathrm{U}<\mathrm{EQL} \quad \mathrm{ug} / \mathrm{L}$

$<10 \quad U \quad<E Q L \quad u g / L$

$<20 \quad U \quad<E Q L \quad \mathrm{ug} / \mathrm{L}$

$<3.43 \quad \mathrm{JU} \quad<\mathrm{EQL} \quad \mathrm{ug} / \mathrm{L}$

$<9.5 \quad U<\mathrm{EOL} \quad \mathrm{ug} / \mathrm{G}$

$\mathrm{ug} / \mathrm{L}$

$u g / L$
$u g / L$

$u g / L$

ug/L

$<5 \quad \mathrm{E} \quad \mathrm{EQL} \quad \mathrm{ug} / \mathrm{h}$

$<5 \quad U \quad<E Q L \quad u g / L$

$<5 \quad U<E Q L \quad u g / L$

$<4.7 \quad U \quad<E Q L \quad u g / L$

$\mathrm{ug} / \mathrm{L}$

$\mathrm{ug} / \mathrm{L}$

$\mathrm{ug} / \mathrm{L}$

$\mathrm{ug} / \mathrm{L}$

$\mathrm{ug} / \mathrm{L}$

ug $/ L$

$u g / L$

$\operatorname{ug} / L$

$\mathrm{ug} / \mathrm{L}$

$\mathrm{ug} / \mathrm{L}$

Notes: Concentrations in bold Italics exceed the groundwater protection standards listed in Appendix A. Bold italics were not assigned if the result was qualified with a "J", "R", "L", or "U" qualifler.

$+=$ exceeded the Groundwater Protection Standards listed in Appendix A. 
Table B. Assessment Monitoring Results for Groundwater Wells (Cont.)

WELL LFW 32

ANALYTICAL DATA

Groundwater Protection Standard

ST Parameter

Endrin aldehyde

Ethyl methacrylate

Ethyl methanesulfonate

Ethylbenzene

Famphur

Fluoranthene

Fluorene

Heptachlor

Heptachlor epoxide

Hexachlorobenzene

Hexachlorobutadiene

Hexachlorocyclopentadiene

Hexachloroethane

Hexachloropropene

Indeno(1,2,3-c,d)pyrene

ladomethane (Methyl iodide)

Isobutyl alcohol

Isodrin

isophorone

Isosafrole

Kepone

Lead, total recoverable

Lindane

Mercury, total recoverable

Methacrylonitrile

Methapyrilene

Methoxychlor

Methyl ethyl ketone

Methyl isobutyl ketone

Methyl methacrylate

Methyl methanesulfonate

$\mathrm{N}$-Nitrosodi-n-butylamine

$\mathrm{N}$-Nitrosodiethylamine

$\mathrm{N}$-Nitrosodimethylamine

$\mathrm{N}$-Nitrosodiphenylamine

$\mathrm{N}$-Nitrosodipropylamine

$\mathrm{N}-$ Nitrosomethylethylamine

$\mathrm{N}$-Nitrosopiperidine

$\mathrm{N}$-Nitrosopyrrolidine

Naphthalene

Nickel, total recoverable

Nitrobenzene

O,0,0-Triethyl phosphorothioate

$\mathrm{PCB}$

PCB 1016

PCB 1232

PCB 1242

PCB 1248

PCB 1254

PCB 1260

Parathion

Parathion methyl

Pentachlorobenzene

Pentachloronitrobenzene

Pentachlorophenol

Phenacetin

Phenanthrene

Phenol

Phorate

Pronamid

Propionitrile

Pyrene

Safrole

Selenium, total recoverable

Silver, total recoverable

Specific conductance

Styrene

Sulfide

\begin{tabular}{|c|c|c|c|}
\hline الفة & CLP & & Filt. \\
\hline$<.21$ & U & & $<\mathrm{EQL}$ \\
\hline$<5$ & U & & $<E Q L$ \\
\hline$<11$ & JU & $Q$ & $<E Q L$ \\
\hline$<5$ & U & & $<E Q$ \\
\hline$<200$ & JU & $Q$ & $<\mathrm{EQ}$ \\
\hline$<11$ & JU & $Q$ & $<E Q$ \\
\hline$<11$ & JU & $\vec{Q}$ & $<\mathrm{EQ}$ \\
\hline$<.1$ & U & & $<\mathrm{EQ}$ \\
\hline$<.1$ & $\mathrm{U}$ & & $<E Q$ \\
\hline$<11$ & JU & $Q$ & $<\mathrm{EQ}$ \\
\hline$<11$ & JU & $Q$ & $<E Q$ \\
\hline$<11$ & $\mathrm{JU}$ & $Q$ & $<E Q$ \\
\hline$<11$ & JU & $Q$ & $<E Q$ \\
\hline$<53$ & JU & $\mathbf{Q}$ & $<\mathrm{EQ}$ \\
\hline$<11$ & JU & $Q$ & $<$ EQL \\
\hline$<5$ & U & & $<\mathrm{EQ}$ \\
\hline$<500$ & $\mathrm{U}$ & & $<\mathrm{EQ}$ \\
\hline$<11$ & JU & $Q$ & $<$ EQL \\
\hline$<11$ & JU & $Q$ & $<\mathrm{EQL}$ \\
\hline$<11$ & JU & $Q$ & $<$ EQL \\
\hline$<11$ & JU & $Q$ & $<E Q L$ \\
\hline$<2.88$ & $\mathrm{JU}$ & & $<E Q$ \\
\hline$<.1$ & $U$ & & $<E Q$ \\
\hline$<.37$ & $U$ & V & $<E Q L$ \\
\hline$<200$ & $\mathbf{U}$ & & $<\mathrm{EQL}$ \\
\hline$<11$ & $J U$ & $Q$ & $<$ EQL \\
\hline$<1$ & $U$ & & $<\mathrm{EQL}$ \\
\hline$<20$ & $U$ & & $<$ EQL \\
\hline$<10$ & U & & $<E Q L$ \\
\hline$<20$ & U & & $<\mathrm{EQL}$ \\
\hline$<11$ & JU & $Q$ & $<E Q L$ \\
\hline$<11$ & JU & $Q$ & $<E Q$ \\
\hline$<11$ & JU & $Q$ & $<E Q L$ \\
\hline$<26$ & JU & $Q$ & $<\mathrm{EQL}$ \\
\hline$<11$ & JU & $Q$ & $<E Q L$ \\
\hline$<11$ & JU & $Q$ & $<\mathrm{EQL}$ \\
\hline$<11$ & JU & $\mathrm{Q}$ & $<\mathrm{EQ}$ \\
\hline$<11$ & JU & $Q$ & $<$ EQL \\
\hline$<11$ & JU & $Q$ & $<$ EQL \\
\hline$<11$ & JU & $Q$ & $<\mathrm{EQ}$ \\
\hline$<50$ & $U$ & & $<\mathrm{EQ}$ \\
\hline$<11$ & JU & $Q$ & $<\mathrm{EQ}$ \\
\hline$<11$ & JU & $\mathbf{Q}$ & $<E Q$ \\
\hline$<1$ & $\mathrm{U}$ & & $<\mathrm{EQ}$ \\
\hline$<1$ & U & & $<\mathrm{EQ}$ \\
\hline$<2.1$ & $U$ & & $<E Q$ \\
\hline$<1$ & $U$ & & $<E Q$ \\
\hline$<1$ & U & & $<\mathrm{EQ}$ \\
\hline$<1$ & $U$ & & $<\mathrm{EQ}$ \\
\hline$<11$ & JU & $Q$ & $<E Q$ \\
\hline$<11$ & JU & $Q$ & $<\mathrm{EQ}$ \\
\hline$<53$ & JU & $Q$ & $<\mathrm{EQ}$ \\
\hline$<11$ & JU & $Q$ & $<\mathrm{EQ}$ \\
\hline$<26$ & JU & $Q$ & $<E Q$ \\
\hline$<11$ & JU & $Q$ & $<\mathrm{EQ}$ \\
\hline$<11$ & JU & $Q$ & $<\mathrm{EQ}$ \\
\hline$<11$ & JU & $Q$ & $<E Q$ \\
\hline$<11$ & JU & $Q$ & $<\mathrm{EQL}$ \\
\hline$<11$ & JU & $Q$ & $<\mathrm{EQ}$ \\
\hline$<200$ & U & & $<\mathrm{EQ}$ \\
\hline$<11$ & JU & $Q$ & $<E Q 1$ \\
\hline$<11$ & JU & $Q$ & $<\mathrm{EQL}$ \\
\hline$<10$ & U & & $<E Q$ \\
\hline$<20$ & $U$ & & $<\mathrm{EQ}$ \\
\hline 25 & & & $<37$ \\
\hline$<5$ & U & & $<\mathrm{EQ}$ \\
\hline 1000 & $\mathbf{R}$ & $Q$ & Reject \\
\hline
\end{tabular}

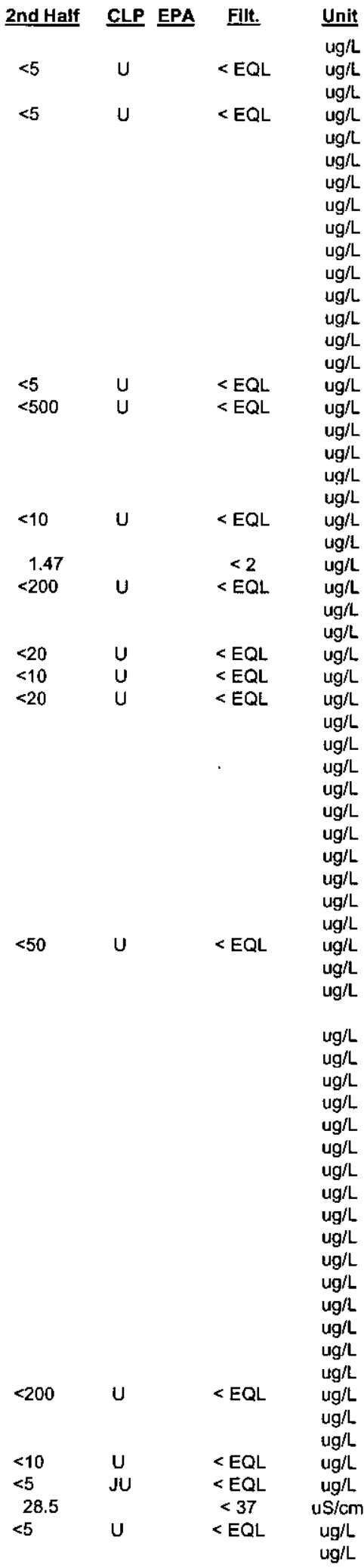

Notes: Concentrations in bold italics exceed the groundwater protection standards listed in Appendix A. Bold italics were not assigned if the result was qualified with a "J", "R", "L", or "U" qualifier.

$+=$ exceeded the Groundwater Protection Standards listed in Appendix A. 
Table B. Assessment Monitoring Results for Groundwater Welis (Cont.)

WELL LFW 32

ANALYTICAL DATA

Groundwater Protection Standard

\section{ST Parameter}

Tetrachloroethylene

Thallium, total recoverable

Thionazin

Tin, total recoverable

Toluene

Toxaphene

Trichloroethylene

Trichlorofluoromethane

Vanadium, total recoverable

Vinyl acetate

Xylenes

Zinc, total recoverable

alpha-Benzene hexachloride

alpha-Chlordane

beta-Benzene hexachloride

cis-1,2-Dichloroethylene

cis-1,3-Dichloropropene

delta-Benzene hexachloride

gamma-Chlordane

m-Cresol (3-Methylphenol)

$\mathrm{m}-$ Nitroaniline

o-Cresol (2-Methylphenol)

o-Nitroaniline

o-Toluidine

$p, p "-D D D$

p.p"-DDE

p.p"-DDT

p-Cresol (4-Methylphenol)

p-Dimethylaminoazobenzene

p-Nitroaniline

p-Phenylenediamine

$\mathrm{pH}$

trans-1,2-Dichloroethylene

trans-1,3-Dichloropropene

trans-1,4-Dichloro-2-butene

\begin{tabular}{|c|c|c|c|}
\hline 1 st Half & CLP & EPA & Filt. \\
\hline$<5$ & $\mathrm{U}$ & & $<E Q L$ \\
\hline$<10$ & $U$ & & $<$ EQL \\
\hline$<11$ & JU & $Q$ & $<$ EQL \\
\hline$<200$ & $U$ & & $<\mathrm{EQL}$ \\
\hline$<5$ & $\mathrm{U}$ & & $<$ EQL \\
\hline$<2.1$ & $U$ & & $<$ EQL \\
\hline .91 & $J$ & 1 & NDD \\
\hline 160 & & & $>20$ \\
\hline$<10$ & $U$ & & $<E Q L$ \\
\hline$<5$ & $U$ & & $<\mathrm{EQL}$ \\
\hline$<10$ & $U$ & & $<\mathrm{EQL}$ \\
\hline$<7.99$ & JU & & $<E Q L$ \\
\hline$<.1$ & $U$ & & $<$ EQL \\
\hline$<.1$ & $U$ & & $<\mathrm{EQL}$ \\
\hline$<.1$ & $U$ & & $<E Q L$ \\
\hline$<5$ & $U$ & & $<E Q L$ \\
\hline$<5$ & $U$ & & $<E Q L$ \\
\hline$<.1$ & U & & $<E Q L$ \\
\hline$<.1$ & $U$ & & $<E Q L$ \\
\hline$<26$ & JU & $Q$ & $<\mathrm{EQL}$ \\
\hline$<11$ & JU & $Q$ & $<E Q L$ \\
\hline$<26$ & JU & $\mathbf{Q}$ & $<E Q L$ \\
\hline$<11$ & JU & $Q$ & $<E Q L$ \\
\hline$<.21$ & $\mathbf{U}$ & & $<E Q L$ \\
\hline$<.21$ & $\mathbf{U}$ & & $<$ EQL \\
\hline$<.21$ & $U$ & & $<E Q L$ \\
\hline$<10$ & JU & $Q$ & $<E Q L$ \\
\hline$<11$ & JU & $Q$ & $<E Q L$ \\
\hline$<11$ & JU & $\mathbf{Q}$ & $<\mathrm{EQL}$ \\
\hline$<11$ & JU & $Q$ & $<E Q L$ \\
\hline 5.8 & $J$ & $Q$ & NDD \\
\hline$<5$ & $\mathbf{U}$ & & $<\mathrm{EQL}$ \\
\hline$<5$ & $U$ & & $<E Q L$ \\
\hline$<20$ & $U$ & & $<E Q L$ \\
\hline
\end{tabular}

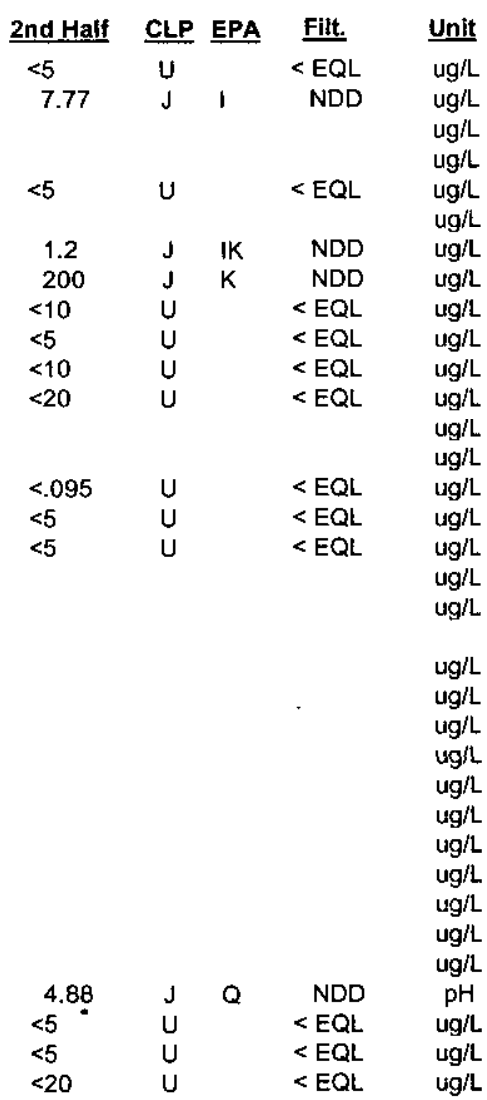

Notes: Concentrations in bold italics exceed the groundwater protection standards listed in Appendix A. Bold italtcs were not assigned if the result was qualified with a "J", "R", "L", or "U" qualifier.

$+=$ exceeded the Groundwater Protection Standards listed in Appendix A. 
Table B. Assessment Monitoring Results for Groundwater Wells (Cont.)

WELL LFW 32C

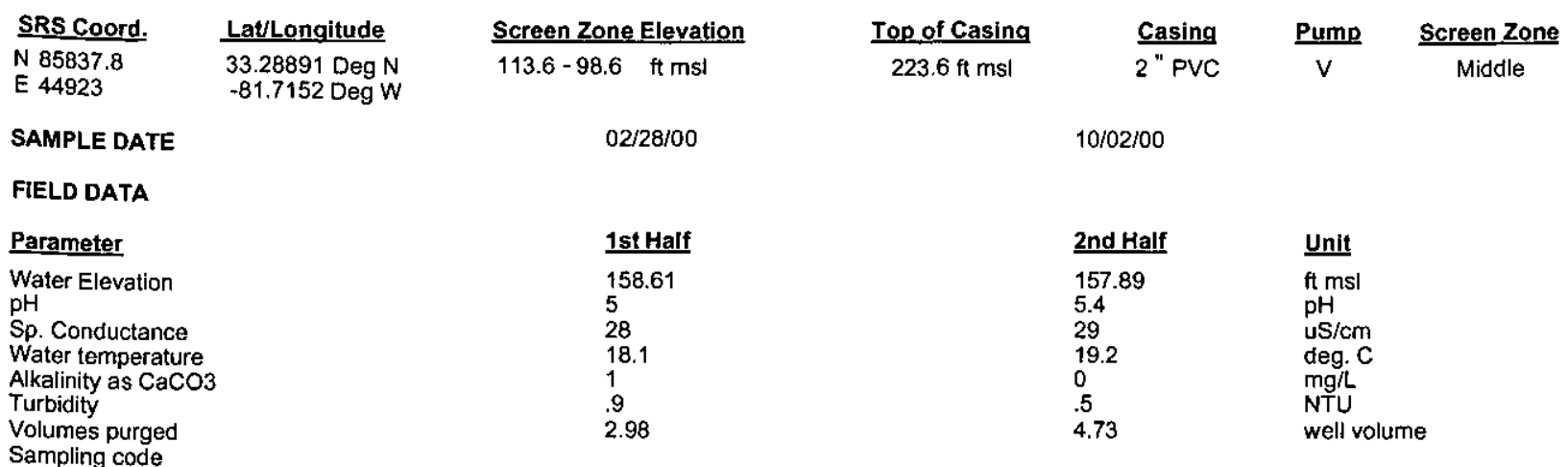

\section{ANALYTICAL DATA}

Groundwater Protection Standard

SI Parameter

1,1,1,2-Tetrachloroethane

1,1,1-Trichloroethane

$1,1,2,2$-Tetrachloroethane

1,1,2-Trichloroethane

1,1-Dichloroethane

1,1-Dichloroethylene

1,1-Dichloropropene

1,2,3-Trichloropropane

1,2,4,5-Tetrachlorobenzene

1,2,4-Trichlorobenzene

1,2-Dibromo-3-chloropropane

1,2-Dibromoethane

1,2-Dichlorobenzene

1,2-Dichloroethane

1,2-Dichloropropane

1,3,5-Trinitrobenzene

1,3-Dichlorobenzene

1,3-Dichloropropane

1,3-Dinitrobenzene

1,4-Dichlorobenzene

1.4-Naphthoquinone

1-Naphthylamine

2,2-Dichloropropane

2,2-Oxybis (1-chloropropane)

2,3,4,6-Tetrachlorophenol

$2,4,5-\mathrm{T}$

2,4,5-TP (Silvex)

2,4,5-Trichlorophenol

2,4,6-Trichlorophenol

2,4-Dichlorophenol

2,4-Dichlorophenoxyacetic acid

2,4-Dimethyl phenol

2,4-Dinitrophenol

2,4-Dinitrotoluene

2,6-Dichlorophenol

2,6-Dinitrotoluene

2-Acetylaminofluorene

2-Chloronaphthalene

2-Chlorophenol

2-Hexanone

2-Methyl-4,6-dinitrophenol

2-Methylnaphthalene

2-Naphthylamine

2-Nitrophenol

2-sec-Butyl-4,6-dinitrophenol

3,3"-Dichlorobenzidine

3,3"-Dimethylbenzidine

3-Methylcholanthrene

4-Aminobiphenyl

4-Bromophenyl phenyl ether

1st Half CLP EPA Filt

$\begin{array}{lll}<5 & U & <E Q\end{array}$

U $<$ EQL

$\begin{array}{lll}<5 & U & <E Q L \\ <5 & U & <E Q L\end{array}$

$<5 \quad U \quad<E Q L$

$<5 \quad U \quad<E Q L$

$<5 \quad U<$ <QL

$<5 \quad U<E Q L$

$<52$ JU L $<E Q L$

$<11$ JU L $<E Q L$

$<10 \quad U<E Q L$

$<5 \quad U<$ EQL.

$<11$ JU $\mathrm{L}<\mathrm{EQL}$

$<5 \quad U \quad<E Q L$

$<5 \quad U<E Q L$

$<10$ JU $\mathrm{L} \quad<$ EQL

$<5 \quad U<$ \&QL

$<5 \quad U<E Q L$

$<50 \quad J U$ L $<E Q L$

$<5 \quad U<E Q L$

$<11$ JU L < EQL

$<11$ JU L $<E Q L$

$<5 \quad U \quad<E Q L$

$<11$ JU L $<$ EQL

$<11$ JU L $<$ EQL

$<.2 \quad U<$ EQL

$<2 \quad U$

$<11$ JU L $<$ EQL

$<26$ JU $L \quad<E Q L$

$<11$ JU $\mathrm{L}<\mathrm{EQL}$

$<.2 \quad U<$ U $<Q$

$<11$ JU $\mathrm{L} \quad<\mathrm{EQL}$

$<26$ JU L $<E Q L$

$<11$ JU L $<$ EQL

$<25<11<\mathrm{JU}$

$<11$ JU L $<E Q L$

$<11$ JU L $<$ EQL

$<11$ JU L

$<11$ JU $L \quad<E Q L$

$<20 \quad \mathrm{~J}<\mathrm{EQL}$

$<26$ JU L $<$ EQL

$<11$ JU L $<$ EQL

$<11<$ JU L $<$ EQL

$<11$ JU L $<$ EQL

$<.2$ JU $\mathrm{L} \quad<\mathrm{EQL}$

$<11$ JU $L \quad<$ EQL

$<21$ JU L $<$ EQL

$<11$ JU $\mathrm{L}<\mathrm{EQL}$

$<11$ JU $\mathrm{L}<\mathrm{EQL}$

$<11$ JU L $<$ EQL

$<11$ JU L < EQL 2nd Half CLP EPA Filt. Unit

$<5 \quad U \quad<E Q L \quad u g / L$

$<5 \quad U \quad<E Q \quad \mathrm{ug} / \mathrm{L}$

$<5 \quad U \quad<E Q L \quad \mathrm{ug} / \mathrm{L}$

$<5 \quad U \quad<$ EQL ug/L

$<5 \quad U<E Q L \quad u g / L$

$<5 \quad \mathrm{U} \quad<\mathrm{EQL} \quad \mathrm{ug} / \mathrm{L}$

$<5 \quad U \quad<E Q \quad$ ug/L

$<5 \quad U \quad<$ EQL ug/L

ug/L

$\begin{array}{llll}<10 & \mathrm{U} & <\mathrm{EQL} & \mathrm{ug} / \mathrm{L} \\ <5 & \mathrm{U} & <\mathrm{EQL} & \mathrm{ug} / \mathrm{L}\end{array}$

$<5 \quad U \quad<E Q$ ug/L

$<5 \quad U \quad<E Q \quad$ ug/L

$<5 \quad U \quad<E Q L \quad u g / L$

$<5 \quad U \quad<E Q L \quad \mathrm{Ug} / \mathrm{L}$

$<5 \quad U \quad<$ EQL Ug/L

$<5 \quad \mathrm{U} \quad<\mathrm{EQL} \quad \mathrm{ug} / \mathrm{L} / \mathrm{ug}$

ug/L

$<5 \quad U \quad<$ EQL ug/L

$\mathrm{ug} / \mathrm{L}$

$u g / L$

ug/L

$u g / L$

$u g / L$

ug/L

ug/L

$\mathrm{ug} / \mathrm{L}$

$u g / L$

$\mathrm{ug} / \mathrm{L}$

ug/L

$\mathrm{ug} / \mathrm{L}$

$u g / L$

$\mathrm{ug} / \mathrm{L}$

$\mathrm{ug} / \mathrm{L}$

$u g / L$

$u g / L$

$\mathrm{ug} / \mathrm{L}$

ug/L

$u g / L$

ug/h

ug/L

$u g / L$

ug/L

ug/L

$u g / L$

Notes: Concentrations in bold italics exceed the groundwater protection standards listed in Appendix A. Bold italics were not assigned if the result was qualified with a "J", "R", "L", or "U" qualifier.

$+=$ exceeded the Groundwater Protection Standards listed in Appendix A. 


\section{ANALYTICAL DATA}

Groundwater Protection Standard

\section{SI Parameter}

4-Chloroaniline

4-Chlorophenyl phenyl ether

4-Nitrophenol

5-Nitro-o-toluidine

7,12-Dimethylbenz(a)anthracene

Acenaphthene

Acenaphthylene

Acetone

Acetonitrile (Methyl cyanide)

Acetophenone

Acrolein

Acrylonitrile

Aldrin

Allyl chloride

Anthracene

Antimony, total recoverable

Arsenic, total recoverable

Barium, total recoverable

Benzene

Benzo(a)anthracene

Benzo(a)pyrene

Benzo(b)fluoranthene

Benzo(g,h,i)perylene

Benzo(k)fluoranthene

Benzyl alcohol

Beryllium, total recoverable

Bis(2-chloroethoxy) methane

Bis(2-chloroethyl) ether

Bis(2-ethylhexyl) phthalate

Bromochloromethane

Bromodichloromethane

Bromoform

Bromomethane (Methyl bromide)

Butylbenzyl phthalate

Cadmium, total recoverable

Carbon disulfide

Carbon tetrachloride

Chlorobenzene

Chlorobenzilate

Chloroethane

Chloroethene (Vinyl chloride)

Chloroform

Chloromethane (Methyl chloride)

Chloroprene

Chromium, total recoverable

Chrysene

Cobalt, total recoverable

Copper, total recoverable

Cyanide

Di-n-butyl phthalate

Di-n-octyl phthalate

Diallate

Dibenz(a,h)anthracene

Dibenzofuran

Dibromochloromethane

Dibromomethane (Methylene bromide)

Dichlorodifluoromethane

Dichloromethane (Methylene chloride)

Dieldrin

Diethyl phthalate

Dimethoate

Dimethyl phthalate

Diphenylamine

Disulfoton

Endosulfan I

Endosulfan II

Endosulfan sulfate

Endrin

\begin{tabular}{|c|c|c|c|}
\hline Ist Half & CLP & EPA & Filt. \\
\hline$<11$ & JU & $L$ & $<E Q L$ \\
\hline$<11$ & JU & $\mathrm{L}$ & $<E Q L$ \\
\hline$<26$ & JU & $\mathrm{L}$ & $<\mathrm{EQL}$ \\
\hline$<11$ & JU & $\mathrm{L}$ & $<\mathrm{EQ}$ \\
\hline$<11$ & JU & $\bar{L}$ & $<E Q$ \\
\hline$<11$ & JU & $L$ & $<\mathrm{EC}$ \\
\hline$<11$ & JU & $\bar{L}$ & $<E$ \\
\hline$<20$ & U & & $<E C$ \\
\hline$<200$ & $u$ & & $<E C$ \\
\hline$<11$ & $\mathrm{JU}$ & $L$ & $<E$ \\
\hline$<50$ & U & & $<E$ \\
\hline$<10$ & U & & $<\mathrm{EC}$ \\
\hline$<.11$ & U & & $<\mathrm{E}$ \\
\hline$<5$ & U & & $<\mathrm{E}$ \\
\hline$<11$ & JU & L & $<\mathrm{EC}$ \\
\hline$<100$ & U & & $<\bar{E}$ \\
\hline$<10$ & $U$ & & $<\mathrm{EC}$ \\
\hline 5.78 & $\mathrm{~J}$ & 1 & $T$ \\
\hline$<5$ & U & & $<\mathrm{E}$ \\
\hline$<11$ & JU & L & $<E C$ \\
\hline$<11$ & JU & L & $<E$ \\
\hline$<11$ & JU & $\mathbf{L}$ & $<\mathrm{E}$ \\
\hline$<11$ & JU & L & $<E$ \\
\hline$<11$ & $\mathrm{JU}$ & $\mathrm{L}$ & $<E$ \\
\hline$<.14$ & JU & V & $<\mathrm{E}$ \\
\hline$<11$ & JU & L & $<$ \\
\hline$<11$ & JU & $L$ & $<E$ \\
\hline$<11$ & JU & L & $<$ \\
\hline$<5$ & $U$ & & \\
\hline$<5$ & $U$ & & \\
\hline$<5$ & U & & \\
\hline$<5$ & U & & \\
\hline$<11$ & JU & L & \\
\hline$<10$ & $U$ & & \\
\hline$<5$ & U & & \\
\hline$<5$ & U & & \\
\hline$<5$ & $u$ & & \\
\hline$<11$ & JU & L & \\
\hline$<5$ & $U$ & & \\
\hline$<5$ & $u$ & & $<$ \\
\hline$<5$ & U & & $1<$ \\
\hline$<5$ & U & & \\
\hline$<20$ & U & & \\
\hline$<10$ & U & & $<$ \\
\hline$<11$ & JU & L & $<$ \\
\hline$<20$ & $u$ & & \\
\hline$<20$ & U & & \\
\hline$<10$ & U & & \\
\hline$<11$ & JU & L & \\
\hline$<11$ & JU & L & \\
\hline$<11$ & JU & L & \\
\hline$<11$ & $\mathrm{JU}$ & L & \\
\hline$<11$ & JU & L & \\
\hline$<5$ & U & & \\
\hline$<5$ & U & & \\
\hline$<5$ & U & & \\
\hline$<2.7$ & U & V & $<$ \\
\hline$<.21$ & U & & \\
\hline$<11$ & JU & L & \\
\hline$<11$ & JU & L & \\
\hline$<11$ & JU & L & \\
\hline$<11$ & JU & L & \\
\hline$<11$ & JU & L & $<$ \\
\hline$<.11$ & U & & \\
\hline$<.21$ & $U$ & & \\
\hline$<.21$ & U & & 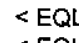 \\
\hline
\end{tabular}

2nd Half CLP EPA Filt. Unit

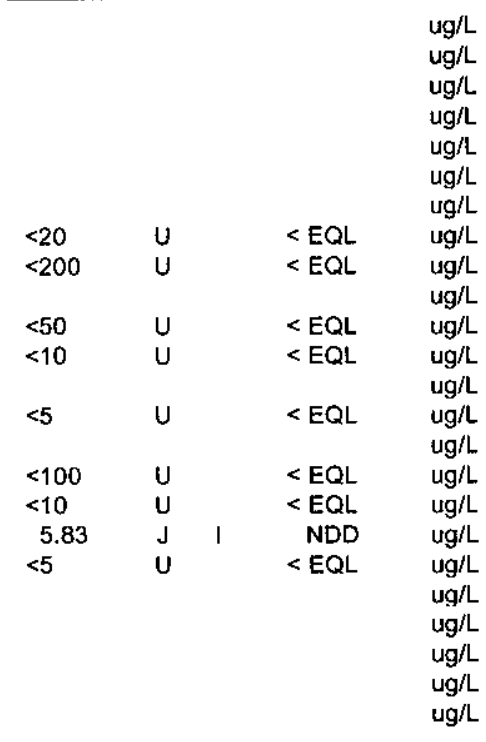

$<19 \quad \mathrm{JU} \quad<\mathrm{EQL} \quad \mathrm{ug} / \mathrm{h}$

$u g / L$

$\mathrm{ug} / \mathrm{L}$

$\begin{array}{llll}<5 & U & <E Q L & \mathrm{ug} / \mathrm{L} \\ <5 & \mathrm{Ug} / \mathrm{L} \\ & \mathrm{U} & <\mathrm{EQL} & \mathrm{ug} / \mathrm{L}\end{array}$

$<5 \quad U \quad<E Q \quad$ ug/L

$<5 \quad U \quad<E Q L \quad u g / L$

$<10 \quad \mathrm{U} \quad<$ EQL ug/L

$<5 \quad U \quad<E Q L \quad$ ug/L

$<5 \quad U \quad<E Q L \quad u g / L$

$<5 \quad U<E Q \quad \mathrm{ug} / \mathrm{L}$

$<5 \quad \mathrm{U} \quad<\mathrm{EQL} \quad \mathrm{ug} / \mathrm{L}$

$<5 \quad U<E Q L \quad u g / L$

$<5 \quad U \quad<E Q L \quad u g / L$

$<5 \quad U \quad<E Q L \quad$ ug/L

$<20 \quad \mathrm{U} \quad<$ EQL ug/L

$<10 \quad U \quad<$ EQL ug/L

$<20 \quad U \quad<E Q \quad \mathrm{ug} / \mathrm{L}$

$<20 \quad U \quad<$ EQL ug/L

$<9.6 \quad U<E Q \quad \mathrm{ug} / \mathrm{L}$

$\mathrm{ug} / \mathrm{L}$

$\mathrm{ug} / \mathrm{L}$

ug/L

ug/L

ug/L

$\mathrm{ug} / \mathrm{L}$

$\mathrm{ug} / \mathrm{h}$

ug/L

$\mathrm{ug} / \mathrm{L}$

$u g / L$

ug/L

$\mathrm{ug} / \mathrm{L}$

$\mathrm{ug} / \mathrm{L}$

ug/L

$\mathrm{ug} / \mathrm{L}$

$u g / L$

ug/L

ug/L

Notes: Concentrations in bold italics exceed the groundwater protection standards listed in Appendix A. Bold italics were not assigned if the result was qualified with a " $J$ ", "R", "L", or "U" qualifier.

$+=$ exceeded the Groundwater Protection Standards listed in Appendix A. 
Table B. Assessment Monitoring Results for Groundwater Wells (Cont.)

WELL LFW 32C

ANALYTICAL DATA

Groundwater Protection Standard

ST Parameter

Endrin aldehyde

Ethyl methacrylate

Ethyl methanesulfonate

Ethylbenzene

Famphur

Fluoranthene

Fluorene

Heptachlor

Heptachlor epoxide

Hexachlorobenzene

Hexachlorobutadiene

Hexachlorocyclopentadiene

Hexachloroethane

Hexachloropropene

Indeno(1,2,3-c,d)pyrene

lodomethane (Methyl iodide)

isobutyl alcohol

Isodrin

Isophorone

Isosafrole

Kepone

Lead, total recoverable

Lindane

Mercury, total recoverable

Methacrylonitrile

Methapyrilene

Methoxychlor

Methyl ethyl ketone

Methyl isobutyl ketone

Methyl methacrylate

Methyl methanesulfonate

$\mathrm{N}$-Nitrosodi-n-butylamine

$\mathrm{N}$-Nitrosodiethylamine

$\mathrm{N}$-Nitrosodimethylamine

$\mathrm{N}$-Nitrosodiphenylamine

N-Nitrosodipropylamine

$\mathrm{N}$-Nitrosomethylethylamine

$\mathrm{N}$-Nitrosopiperidine

$\mathrm{N}$-Nitrosopyrrolidine

Naphthalene

Nickel, total recoverable

Nitrobenzene

$0,0,0$-Triethyl phosphorothioate

$P C B$

PCB 1016

PCB 1232

$\mathrm{PCB} 1242$

PCB 1248

PCB 1254

PCB 1260

Parathion

Parathion methyl

Pentachlorobenzene

Pentachloronitrobenzene

Pentachlorophenol

Phenacetin

Phenanthrene

Phenol

Phorate

Pronamid

Propionitrile

Pyrene

Safrole

Selenium, total recoverable

Silver, total recoverable

Specific conductance

Styrene

Sulfide

\begin{tabular}{|c|c|c|c|}
\hline Hall & CLP & EPA & Filt. \\
\hline 21 & $U$ & & $<\mathrm{EQL}$ \\
\hline & $U$ & & $<\mathrm{EQL}$ \\
\hline & JU & L & $<E Q L$ \\
\hline & U & & $<E Q L$ \\
\hline 00 & JU & $\mathbf{L}$ & $<\mathrm{EQL}$ \\
\hline 1 & JU & $\mathbf{L}$ & $<\mathrm{EQL}$ \\
\hline & JU & $\mathrm{L}$ & $<E Q L$ \\
\hline 11 & $U$ & & $<\mathrm{EQL}$ \\
\hline 11 & U & & $<E Q L$ \\
\hline & JU & $\mathrm{L}$ & $<E Q L$ \\
\hline 1 & JU & $\mathrm{L}$ & $<$ EQL \\
\hline 1 & JU & $\mathrm{L}$ & $<E Q L$ \\
\hline & JU & $L$ & $<\mathrm{EQL}$ \\
\hline 2 & JU & $\mathbf{L}$ & $<E Q L$ \\
\hline 1 & JU & $\mathbf{L}$ & $<$ EQL \\
\hline & $\mathrm{U}$ & & $<$ EQL \\
\hline 00 & U & & $<\mathrm{EQL}$ \\
\hline 1 & JU & $L$ & $<\mathrm{EQL}$ \\
\hline 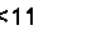 & JU & $L$ & $<E Q L$ \\
\hline & JU & $\mathrm{L}$ & $<E Q L$ \\
\hline 1 & JU & $L$ & $<E Q L$ \\
\hline U & $U$ & & $<E Q L$ \\
\hline 11 & U & & $<E Q L$ \\
\hline & U & & $<$ EQL \\
\hline 00 & U & & $<\mathrm{EQL}$ \\
\hline & JU & $L$ & $<E Q L$ \\
\hline 1 & U & & $<\mathrm{EQL}$ \\
\hline & U & & $<E Q L$ \\
\hline & $\mathrm{U}$ & & $<$ EQL \\
\hline & U & & $<E Q L$ \\
\hline & JU & $L$ & $<E Q L$ \\
\hline & JU & $\mathbf{L}$ & $<E Q L$ \\
\hline & JU & L & $<E Q L$ \\
\hline & JU & L & $<\mathrm{EQL}$ \\
\hline & JU & $L$ & $<E Q L$ \\
\hline & JU & $L$ & $<E Q L$ \\
\hline & JU & $L$ & $<\mathrm{EQL}$ \\
\hline & JU & $L$ & $<E Q L$ \\
\hline & JU & $L$ & $<$ EQL \\
\hline & JU & $L$ & $<E Q L$ \\
\hline & U & & $<E Q L$ \\
\hline & JU & $\mathbf{L}$ & $<$ EQL \\
\hline & JU & $L$ & $<$ EQL \\
\hline & $U$ & & $<$ EQL \\
\hline 1 & $U$ & & $<E Q L$ \\
\hline .1 & $U$ & & $<E Q L$ \\
\hline 1 & $U$ & & $<E Q L$ \\
\hline 1 & $U$ & & $<$ EQL \\
\hline & $U$ & & $<E Q L$ \\
\hline & JU & $\mathbf{L}$ & $<\mathrm{EQL}$ \\
\hline & JU & $L$ & $<E Q L$ \\
\hline 2 & JU & $L$ & $<E Q L$ \\
\hline & JU & $L$ & $<E Q L$ \\
\hline & JU & $L$ & $<E Q L$ \\
\hline & $\mathrm{JU}$ & L & $<E Q L$ \\
\hline & JU & $\mathbf{L}$ & $<\mathrm{EQL}$ \\
\hline & JU & $\mathbf{L}$ & $<$ EQL \\
\hline 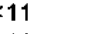 & $\mathrm{JU}$ & $L$ & $<$ EQL \\
\hline 1 & JU & $L$ & $<\mathrm{EQL}$ \\
\hline 200 & $\mathrm{U}$ & & $<E Q L$ \\
\hline 11 & JU & $L$ & $<$ EQL \\
\hline & $\mathrm{JU}$ & $L$ & $<E Q L$ \\
\hline & $U$ & & $<E Q L$ \\
\hline 20 & U & & $<E Q L$ \\
\hline 27.7 & & & $<37$ \\
\hline & U & & $<E Q L$ \\
\hline 100 & U & & $<\mathrm{EQL}$ \\
\hline
\end{tabular}

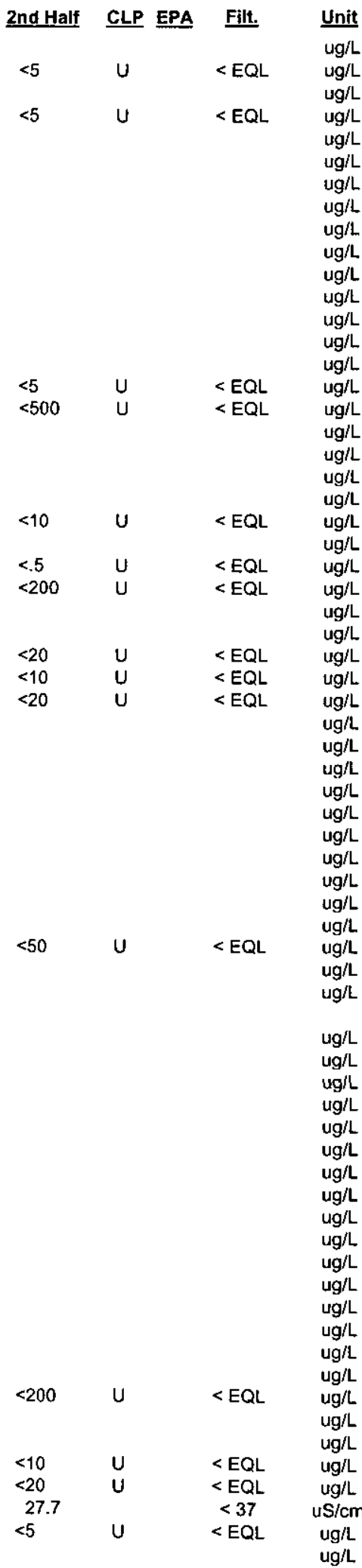

Notes: Concentrations in bold italics exceed the groundwater protection standards listed in Appendix A. Bold italics were not assigned if the result was qualified with a "J", "R", "L", or "U" qualifier.

$+=$ exceeded the Groundwater Protection Standards listed in Appendix A. 
Table B. Assessment Monitoring Results for Groundwater Wells (Cont.)

WELL LFW 32C

\section{ANALYTICAL DATA}

Groundwater Protection Standard

\section{ST Parameter}

Tetrachloroethylene

Thallium, total recoverable

Thionazin

Tin, total recoverable

Toluene

Toxaphene

Trichloroethylene

Trichlorofluoromethane

Vanadium, total recoverable

Vinyl acetate

Xylenes

Zinc, total recoverable

alpha-Benzene hexachloride alpha-Chlordane

beta-Benzene hexachloride

cis-1,2-Dichloroethylene

cis-1,3-Dichloropropene

delta-Benzene hexachloride

gamma-Chlordane

m-Cresol (3-Methylphenol)

m-Nitroaniline

o-Cresol (2-Methylphenol)

o-Nitroaniline

o-Toluidine

p,p"-DDD

$p, p^{\prime \prime}-D D E$

$p, p^{n}-\mathrm{DDT}$

p-Cresol (4-Methylphenol)

p-Dimethylaminoazobenzene

p-Nitroaniline

p-Phenylenediamine

$\mathrm{pH}$

trans-1,2-Dichloroethylene

trans-1,3-Dichloropropene

trans-1,4-Dichloro-2-butene

\begin{tabular}{|c|c|c|c|}
\hline$t$ Half & CLP & EPA & Filt. \\
\hline$<5$ & $U$ & & $<E Q L$ \\
\hline$<10$ & U & & $<E Q L$ \\
\hline$<11$ & JU & L & $<E Q L$ \\
\hline$<200$ & U & & $<E Q$ \\
\hline$<5$ & U & & $<\mathrm{EC}$ \\
\hline$<2.1$ & U & & $<\mathrm{E}$ \\
\hline$<5$ & U & & $<\mathrm{E}$ \\
\hline$<5$ & U & & $<\mathrm{EC}$ \\
\hline$<10$ & U & & $<E$ \\
\hline$<5$ & $U$ & & $<E$ \\
\hline$<10$ & U & & $<E$ \\
\hline$<20$ & $U$ & & $<E$ \\
\hline$<.11$ & U & & $<$ \\
\hline$<.11$ & $u$ & & $<B$ \\
\hline$<.11$ & $u$ & & $<\mathrm{E}$ \\
\hline$<5$ & $u$ & & $<1$ \\
\hline$<5$ & $u$ & & $<E$ \\
\hline$<.11$ & U & & $<$ \\
\hline$<.11$ & U & & \\
\hline$<26$ & JU & $\mathrm{L}$ & $<E$ \\
\hline$<11$ & JU & L & $<$ \\
\hline$<26$ & JU & L & \\
\hline$<11$ & JU & L & $<1$ \\
\hline$<.21$ & u & & \\
\hline$<.21$ & U & & \\
\hline$<.21$ & u & & \\
\hline$<10$ & JU & $L$ & $<$ \\
\hline$<11$ & Ju & L & \\
\hline$<11$ & JU & $\bar{L}$ & $<$ \\
\hline$<11$ & JU & $L$ & \\
\hline 5.55 & J & $Q$ & \\
\hline$<5$ & $U$ & & \\
\hline$<5$ & $u$ & & \\
\hline$<20$ & $U$ & & ? \\
\hline
\end{tabular}

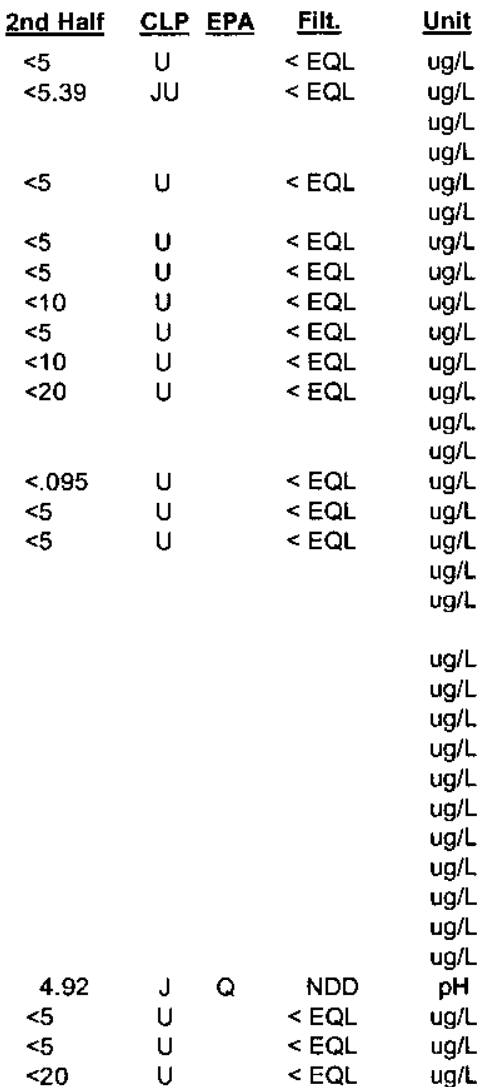

Notes: Concentrations in bold italics exceed the groundwater protection standards listed in Appendix A. Bold ltalics were not assigned if the result was qualified with a "J", "R", "L", or "U" qualifier.

$+=$ exceeded the Groundwater Protection Standards listed in Appendix A. 
Table B. Assessment Monitoring Results for Groundwater Wells (Cont.)

WELL LFW 34

\begin{tabular}{|c|c|}
\hline SRS Coord. & Lat/Longitude \\
\hline $\begin{array}{l}\text { N } 85409.5 \\
\text { E } 45016.9\end{array}$ & $\begin{array}{l}\text { 33.28811 Deg } N \\
-81.7142 \operatorname{Deg} W\end{array}$ \\
\hline
\end{tabular}

SAMPLE DATE

\section{FIELD DATA}

Parameter

Water Elevation

$\mathrm{pH}$

Sp. Conductance

Water temperature

Alkalinity as $\mathrm{CaCO} 3$

Turbidity

Volumes purged

Sampling code

\section{ANALYTICAL DATA}

Groundwater Protection Standard

\section{SI Parameter}

1,1,1,2-Tetrachtoroethane

1,1,1-Trichloroethane

1,1,2,2-Tetrachloroethane

1,1,2-Trichloroethane

1,1-Dichloroethane

1,1-Dichloroethylene

1,1-Dichloropropene

1,2,3-Trichloropropane

1,2,4,5-Tetrachlorobenzene

1,2,4-Trichlorobenzene

1,2-Dibromo-3-chloropropane

1,2-Dibromoethane

1,2-Dichlorobenzene

1,2-Dichioroethane

1,2-Dichloropropane

1,3,5-Trinitrobenzene

1,3-Dichlorobenzene

1,3-Dichloropropane

1,3-Dinitrobenzene

1,4-Dichlorobenzene

1,4-Naphthoquinone

1-Naphthylamine

2,2-Dichloropropane

2,2-Oxybis(1-chloropropane)

2,3,4,6-Tetrachlorophenol

$2,4,5-T$

2,4,5-TP (Silvex)

2,4,5-Trichlorophenol

2,4,6-Trichlorophenol

2,4-Dichlorophenol

2,4-Dichlorophenoxyacetic acid

2,4-Dimethyl phenol

2,4-Dinitrophenol

2,4-Dinitrotoluene

2,6-Dichlorophenol

2,6-Dinitrotoluene

2-Acetylaminofluorene

2-Chloronaphthalene

2-Chlorophenol

2-Hexanone

2-Methyl-4,6-dinitropheno

2-Methylnaphthalene

2-Naphthylamine

2-Nitrophenol

2-sec-Butyl-4,6-dinitrophenol

3,3"-Dichlorobenzidine

3,3"-Dimethybenzidine

3-Methylcholanthrene

4-Aminobiphenyl

4-Bromophenyl phenyl ether

4-Chloro-m-cresol

\author{
Screen Zone Elevation \\ $164.7-143.7 \mathrm{ft} \mathrm{ms}$
}

03/08/00

$\frac{\text { Ist Half }}{158.6}$
5
31
19.5
0
.2
5.60

$<2$

14
$<25$

$<25$
$<25$

$<25$
8.7

$<25$

$<25$

$<25$

$<48$

$<9.6$

$<50$

$<25$

$<25$

$<25$

$<25$

$<10$

$<25$

$<25$

$<50$

$<25$

$<9.6$

$<9.6$

$<25$

$<9.6$

$<9.6$

$<.2$

$<.2$

$<9.6$

$<24$

$<9.6$

$<.2$

$<9.6$

$<24$

$<9.6$

$<25$

$<9.6$

$<9.6$

$<9.6$

$<9.6$

$<100$

$<24$

$<9.6$

$<9.6$

$<.2$

$<9.6$

$<19$

$<9.6$

$<9.6$

$<9.6$

$<9.6$

$\frac{\text { Top of Casing }}{201 \mathrm{ft} \mathrm{msl}}$

$\frac{\text { Casing }}{4 " P V C}$

Pump

$s$

Screen Zone

$08 / 17 / 00$

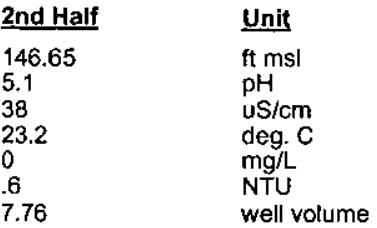

\section{Upper}

Notes: Concentrations in bold italics exceed the groundwater protection standards listed in Appendix A. Bold italics were not assigned if the result was qualified with a "J", "R", "L", or "U" qualifier.

$+=$ exceeded the Groundwater Protection Standards listed in Appendix A.

2ndHalf CLP EPA Filt. Un

$\begin{array}{clll}<5 & U & <E Q L & \mathrm{ug} / \mathrm{L} \\ 15 & & <200 & \mathrm{ug} / \mathrm{L}\end{array}$

$<5 \quad U \quad<E Q \quad$ ug/L

$\begin{array}{llll}<5 & U & <\mathrm{EQL} & \mathrm{ug} / \mathrm{L} \\ & \mathrm{C} & <\mathrm{EQL} & \mathrm{ug} / \mathrm{L}\end{array}$

$<20 \quad \mathrm{ug} / \mathrm{L}$

$<5 \quad U \quad<E Q L \quad u g / L$

$<5 \quad U \quad<E Q \quad U g / L$

$<5 \quad U \quad<$ EQL ug/L

ug/L

$<10 \quad U \quad<$ EQL ug/L

$\begin{array}{llll}<5 & \mathrm{U} & <\mathrm{EQL} & \mathrm{ug} / \mathrm{L} \\ <5 & \mathrm{U} & <\mathrm{EQL} & \mathrm{ug} / \mathrm{L}\end{array}$

$<5 \quad U \quad<E Q \quad u g / L$

$<5 \quad U \quad<$ EQL Ug/L

$<5 \quad u$

$\begin{array}{llll}<5 & \mathrm{U} & <\mathrm{EQL} & \mathrm{ug} / \mathrm{h} \\ <5 & \mathrm{U} & <\mathrm{EQL} & \mathrm{ug} / \mathrm{L}\end{array}$

$\mathrm{ug} / \mathrm{L}$

$<5 \quad U \quad<E Q L \quad u g / L$

$\begin{array}{lllll}<E Q L & <5 & \mathrm{E} & <\mathrm{EQL} & \mathrm{ug} / \mathrm{L} \\ <\mathrm{EQL} & & & & \mathrm{ug} / \mathrm{L}\end{array}$

$<E Q L \quad u g / h$

$<E Q L \quad$ ug/L

$<E Q L \quad$ ug/L

$<E Q L \quad$ ug/L

$\mathrm{ug} / \mathrm{L}$

$<E Q L$

$<$ EQL

$<E Q L$

$<E Q L$

$<$ EQL

$<E Q L$

$<$ EQL

$<E Q L$

$<E Q L$

$<E Q L$

$<E Q L$

$<E Q L$

$<E Q L$

$<$ EQL

$<E Q L$

$<E Q L$

$<E Q L$

$<E Q L$

$<E Q L$

$<$ EQL

$\mathrm{ug} / \mathrm{L}$

ug/L

$\mathrm{ug} / \mathrm{L}$

ug/L

ug/L

$\mathrm{ug} / \mathrm{L}$

ug/L

$\mathrm{ug} / \mathrm{L}$

$<20 \quad U<\mathrm{EQL} \quad \mathrm{ug} / \mathrm{L}$

ug $/ L$

$\mathrm{ug} / \mathrm{L}$

$u g / L$

ug/

ugh

ug/L

ug/L

ug/L

$\mathrm{ug} / \mathrm{L}$

ug/L 
Table B. Assessment Monitoring Results for Groundwater Wells (Cont.)

\section{WELL LFW 34}

\section{ANALYTICAL DATA}

Groundwater Protection Standard

ST Parameter

4-Chloroaniline

4-Chlorophenyl phenyl ether

4-Nitrophenol

5-Nitro-o-toluidine

7,12-Dimethylbenz(a)anthracene

Acenaphthene

Acenaphthylene

Acetone

Acetonitrile (Methyl cyanide)

Acetophenone

Acrolein

Acrylonitrile

Aldrin

Allyl chloride

Anthracene

Antimony, total recoverable

Arsenic, total recoverable

Barium, total recoverable

Bernzene

Benzo(a)anthracene

Benzo(a)pyrene

Benzo(b)fluoranthene

Benzo(g,h,i)perylene

Benzo(k)fluoranthene

Benzyl alcohol

Beryllium, total recoverable

Bis(2-chloroethoxy) methane

Bis(2-chloroethyl) ether

Bis(2-ethylhexyl) phthalate

Bromochloromethane

Bromodichioromethane

Bromoform

Bromomethane (Methyl bromide)

Butylbenzyl phthalate

Cadmium, total recoverable

Carbon disulfide

Carbon tetrachloride

Chlorobenzene

Chlorobenzilate

Chloroethane

Chloroethene (Vinyl chloride)

Chloroform

Chloromethane (Methyl chloride)

Chloroprene

Chromium, total recoverable

Chrysene

Cobalt, total recoverable

Copper, total recoverable

Cyanide

Di-n-butyl phthalate

Di-n-octyl phthalate

Diallate

Dibenz(a,h)anthracene

Dibenzofuran

Dibromochloromethane

Dibromomethane (Methylene bromide)

Dichlorodifluoromethane

Dichloromethane (Methylene chloride)

$\frac{\text { 1st Half }}{<9.6} \frac{\text { CLP }}{\mathrm{JU}} \underset{\mathrm{EPA}}{<\mathrm{Filt.}}$

$<9.6$ JU $Q<E Q L$

$<24$ JU $Q<E Q L$

$<9.6$ JU $Q<E Q L$

$<9.6$ JU $Q<E Q L$

$<9.6$ JU $Q<E Q L$

$<9.6$ JU $Q<E Q$

$<100 \quad U<E Q L$

$<1000 \quad U<E Q L$

$<9.6$ JU $Q<E Q L$

$<250 \quad U \quad<E Q L$

$<50 \quad U \quad<E Q$

$<1 \quad U<E Q$

$<25 \quad U<E Q L$

$<9.6$ JU $Q<E Q L$

$<100 \quad U<E Q L$

$<10 \quad U \quad<E Q$

$16.8<2000$

$<25 \quad U \quad<E Q L$

$<9.6$ JU Q

$\begin{array}{llll}<9.6 & \text { JU } & Q & <E Q L \\ <9.6 & J U & Q & <E Q L\end{array}$

$<9.6$ JU $Q<E O$

$<9.6$ JU $Q<E Q L$

$<1 \quad U<E Q L$

$<9.6$ JU $Q<E Q L$

$<9.6$ JU $Q<E Q L$

$<9.6$ JU $Q<E Q L$

$<25 \quad U \quad<E Q L$

$<25 \quad U<\mathrm{EQL}$

$<25 \quad U<E Q$

$<25 \quad U \quad<E Q$

$<9.6$ JU $Q<E Q L$

$<10 \quad U<E Q$

$<25 \quad U<E Q$

$<25 \quad U<E Q 1$

$<25 \quad U<E Q L$

$<9.6$ JU $Q \quad<E Q L$

$<25 \quad U \quad<E Q L$

$<25 \quad U \quad<E Q L$

$<25 \quad U \quad<E Q L$

$<25 \quad U \quad<E Q L$

$<100 \quad U<E Q L$

$<10 \quad U<E Q$

$<9.6$ JU $Q \quad<E Q L$

$<20 \quad U<$ EQL

$<20 \quad U<E Q L$

10 R $Q$ Rejected

$<9.6 \quad$ JU $Q \quad<E Q L$

$<9.6$ JU $Q<E Q L$

$<9.6$ JU $Q<E Q L$

$<9.6$ JU $Q<E Q$

$<9.6$ JU $Q<E Q L$

$<25 \quad U<E Q L$

$<25 \quad U<E Q L$

$<25 \quad U \quad<E Q L$

10 J I NDD

$<.21 \quad U \quad<$ EQL

$<9.6$ JU $Q<E Q$

$<9.6$ JU $Q<E Q L$

$<9.6$ JU $Q<E Q L$

$<9.6$ JU $Q<E Q L$

$<9.6$ JU $Q<E Q t$

$<.1 \quad U<$ Q $\quad$ EQL

$<21 \quad U<E Q L$

$<.21 \quad U \quad<E Q L$

$<.21 \quad U<E Q$ 2nd Half CLP EPA Filt. Unit

$u g / L$

ug/L

$\mathrm{ug} / \mathrm{L}$

$\operatorname{ug} / L$

$\mathrm{ug} / \mathrm{L}$

ug/L

$\begin{array}{llll}<20 & U & <E Q L & \mathrm{ug} / \mathrm{L} \\ <200 & \mathrm{U} & <\mathrm{EQL} & \mathrm{ug} / \mathrm{L}\end{array}$

ug/L

$<50 \quad U \quad<E Q L \quad u g / L$

$<5 \quad U \quad<E Q L \quad u g / L$

$\begin{array}{llll}<100 & U & <E Q L & u g / L \\ <10 & U & <E Q L & u g / L\end{array}$

$14.5<2000 \quad \mathrm{ug} / \mathrm{h}$

$<5 \quad U \quad<$ EQL ug/L

ug/L

$\mathrm{ug} / \mathrm{L}$

ug/L

$\mathrm{ug} / \mathrm{L}$

$\mathrm{ug} / \mathrm{L}$

$u g / L$

ug/L

$\mathrm{ug} / \mathrm{L}$

$\begin{array}{llll}<5 & \mathrm{U} & <\mathrm{EQL} & \mathrm{ug} / \mathrm{L} \\ <5 & \mathrm{U} & <\mathrm{EQL} & \mathrm{ug} / \mathrm{L}\end{array}$

$<5 \quad U \quad<E Q L \quad u g / L$

$<5 \quad U \quad<E Q L \quad u g / L$

$<10 \quad U \quad<E Q L \quad u g / L$

$<5 \quad U \quad<E Q L \quad u g / L$

$<5 \quad U$

$<5 \quad U<E Q$

4.2 J $\quad$ I $\quad$ NDD $\quad \mathrm{ug} / \mathrm{L}$

$<5 \quad U \mathrm{U} \quad<\mathrm{EQL} \quad \mathrm{ug} / \mathrm{L}$

$<5 \quad \mathrm{U}<\mathrm{EQL}$

$<10 \quad U<\mathrm{EQL} \quad \mathrm{ug} / \mathrm{L}$

$<20 \quad U \quad<\mathrm{EQL} \quad \mathrm{ug} / \mathrm{L}$

$<20 \quad U \quad<E Q L \quad u g / L$

$<5$ JU $0<\mathrm{ug} / \mathrm{L}$

$\mathrm{ug} / \mathrm{L}$

$\mathrm{ug} / \mathrm{L}$

$\mathrm{ug} / \mathrm{L}$

$\mathrm{ug} / \mathrm{L}$

$\mathrm{ug} / \mathrm{L}$

$\mathrm{ug} / \mathrm{L}$

$u g / L$

$u g / L$

$\mathrm{ug} / \mathrm{L}$

$u g / L$

$u g / \mathrm{L}$

ugh

$u g / h$

ugh

ug/L

$u g / L$

ug/L

$\mathrm{ug} / \mathrm{L}$

$\mathrm{ug} / \mathrm{L}$

Notes: Concentrations in bold italics exceed the grounctwater protection standards listed in Appendix A. Bold italics were not assigned if the result was qualified with a "J", "R", "L", or "U" qualifier.

$+=$ exceeded the Groundwater Protection Standards listed in Appendix A 


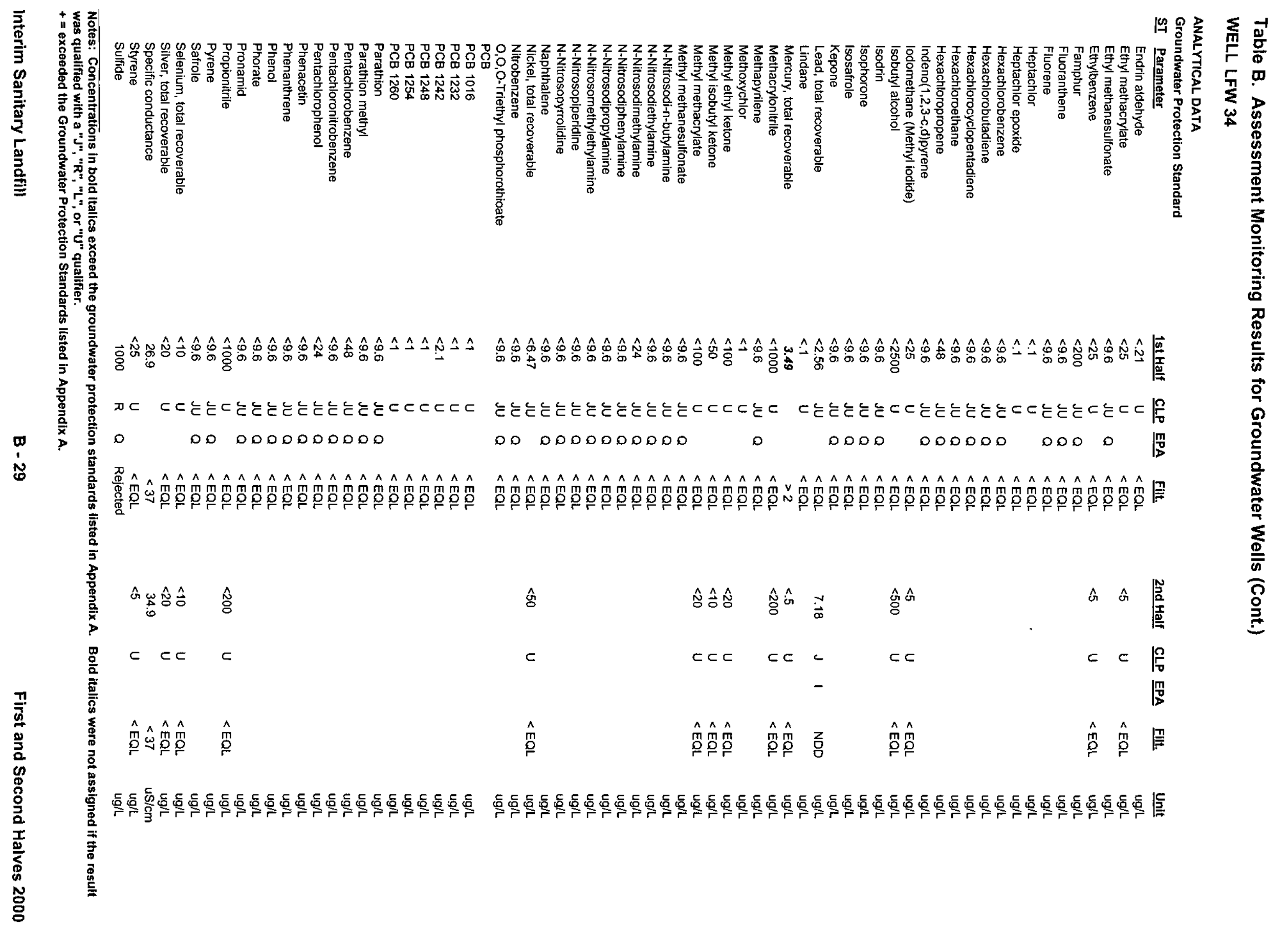

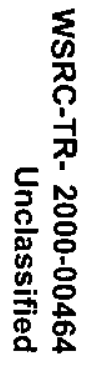


Table B. Assessment Monitoring Results for Groundwater Wells (Cont.)

\section{WELL LFW 34}

ANALYTICAL DATA

Groundwater Protection Standard

\section{ST Parameter}

Tetrachloroethylene

Thallium, total recoverable

Thionazin

Tin, total recoverable

Toluene

Toxaphene

Trichloroethyiene

+ Trichlorofluoromethane

Vanadium, total recoverable

Vinyl acetate

Xylenes

Zinc, total recoverable

alpha-Benzene hexachloride alpha-Chlordane

beta-Benzene hexachloride

cis-1,2-Dichloroethylene

cis-1,3-Dichloropropene

delta-Benzene hexachloride

gamma-Chlordane

m-Cresol (3-Methylphenol)

m-Nitroanifine

o-Cresol (2-Methylphenol)

o-Nitroaniline

o-Toluidine

$p, p=-D D D$

$p, p "-D D E$

p.p"-DDT

p-Cresol (4-Methylphenol)

p-Dimethylaminoazobenzene

p-Nitroaniline

p-Phenylenediamine

pH

trans-1,2-Dichloroethylene

trans-1,3-Dichloropropene

trans-1,4-Dichloro-2-butene

\begin{tabular}{|c|c|c|c|}
\hline 1st Half & $\underline{\text { CLP }}$ & EPA & Filt. \\
\hline$<25$ & U & & $<E Q L$ \\
\hline$<3.34$ &.$J U$ & & $<E Q L$ \\
\hline$<9.6$ & JU & $Q$ & $<\mathrm{EQL}$ \\
\hline$<200$ & U & & $<\mathrm{EQL}$ \\
\hline$<25$ & U & & $<E Q L$ \\
\hline$<2.1$ & U & & $<\mathrm{EQL}$ \\
\hline 2.9 & $J$ & 1 & NOD \\
\hline 490 & & L & $>20$ \\
\hline$<10$ & U & & $<$ EQL \\
\hline$<25$ & U & & $<\mathrm{EQL}$ \\
\hline$<50$ & U & & $<\mathrm{EQL}$ \\
\hline$<20$ & $\mathrm{U}$ & & $<\mathrm{EQL}$ \\
\hline$<.1$ & $U$ & & $<\mathrm{EQL}$ \\
\hline$<.1$ & $U$ & & $<\mathrm{EQL}$ \\
\hline$<.1$ & $U$ & & $<E Q L$ \\
\hline$<25$ & U & & $<\mathrm{EQL}$ \\
\hline$<25$ & U & & $<E Q L$ \\
\hline$<, 1$ & U & & $<E Q L$ \\
\hline$<.1$ & U & & $<E Q L$ \\
\hline$<24$ & JU & $Q$ & $<$ EQL \\
\hline$<9.6$ & JU & $Q$ & $<\mathrm{EQL}$ \\
\hline$<24$ & JU & $Q$ & $<\mathrm{EQL}$ \\
\hline$<9.6$ & JU & $Q$ & $<E Q L$ \\
\hline$<.21$ & U & & $<E Q L$ \\
\hline$<.21$ & $U$ & & $<E Q L$ \\
\hline$<.21$ & U & & $<$ EQL \\
\hline$<10$ & JU & $Q$ & $<E Q L$ \\
\hline$<9.6$ & JU & $Q$ & $<\mathrm{EQL}$ \\
\hline$<9.6$ & JU & $\bar{Q}$ & $<E Q L$ \\
\hline$<9.6$ & JU & $Q$ & $<E Q L$ \\
\hline 5.67 & $\mathrm{~J}$ & 0 & NDD \\
\hline$<25$ & $U$ & & $<\mathrm{EQL}$ \\
\hline$<25$ & $\mathrm{U}$ & & $<\mathrm{EQL}$ \\
\hline$<100$ & $\mathrm{U}$ & & $<\mathrm{EQ}$ \\
\hline
\end{tabular}

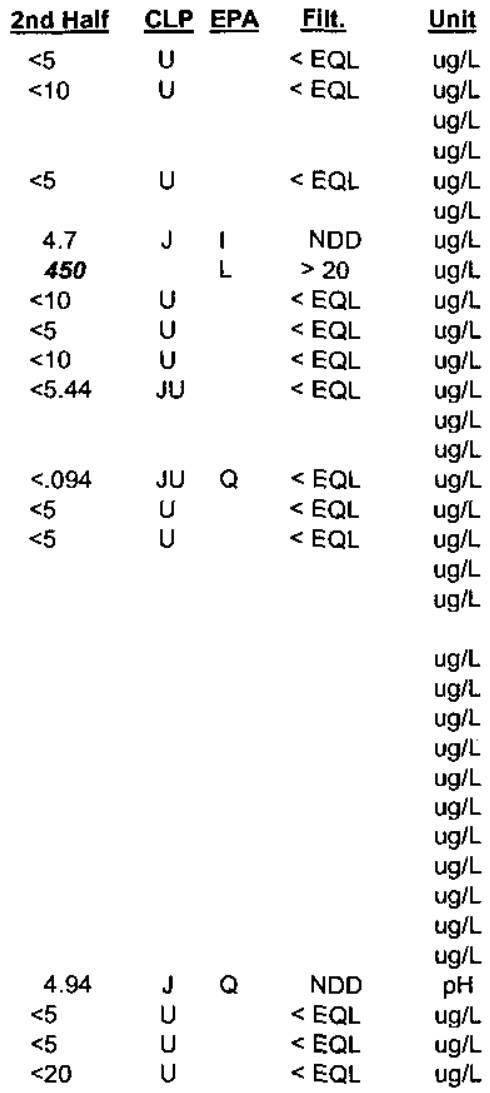

Notes: Concentrations in bold italics exceed the groundwater protection standards listed in Appendix A. Bold italics were not assigned if the result was qualified with a "J", "R", "L", or "U" qualifier.

$+=$ exceeded the Groundwater Protection Standards listed in Appendix A. 
Table B. Assessment Monitoring Results for Groundwater Wells (Cont.)

WELL LFW 43B

$\begin{array}{ll}\text { SRS Coord. } & \text { Lat/Longitude } \\ \text { N } 86459.2 & 33.29080 \mathrm{Deg} N \\ \text { E 45240.5 } & -81.7156 \mathrm{Deg} W\end{array}$

SAMPLE DATE

FIELD DATA

\section{Parameter}

Water Elevation

$\mathrm{pH}$

Sp. Conductance

Water temperature

Alkalinity as $\mathrm{CaCO} 3$

Turbidity

Volumes purged

Sampling code

\section{ANALYTICAL DATA}

Groundwater Protection Standard

ST Parameter

1,1,1,2-Tetrachloroethane

1,1,1-Trichloroethane

1,1,2,2-Tetrachloroethane

1,1,2-Trichloroethane

1,1-Dichloroethane

1,1-Dichloroethylene

1,1-Dichloropropene

1,2,3-Trichloropropane

1,2,4,5-Tetrachlorobenzene

1,2,4-Trichlorobenzene

1,2-Dibromo-3-chloropropane

1,2-Dibromoethane

1,2-Dichlorobenzene

1,2-Dichloroethane

1,2-Dichloropropane

1,3,5-Trinitrobenzene

1,3-Dichlorobenzene

1,3-Dichloropropane

1,3-Dinitrobenzene

1,4-Dichlorobenzene

1,4-Naphthoquinone

1-Naphthylamine

2,2-Dichtoropropane

2,2-Oxybis(1-chloropropane)

2,3,4,6-Tetrachlorophenol

$2,4,5-T$

2,4,5-TP (Silvex)

2,4,5-Trichlorophenol

2,4,6-Trichlorophenol

2,4-Dichlorophenol

2,4-Dichlorophenoxyacetic acid

2,4-Dimethyl phenol

2,4-Dinitrophenol

2,4-Dinitrotoluene

2,6-Dichlorophenol

2,6-Dinitrotoluene

2-Acetylaminofluorene

2-Chloronaphthalene

2-Chlorophenol

2-Hexanone

2-Methyl-4,6-dinitropheno

2-Methyinaphthalene

2-Naphthylamine

2-Nitrophenol

2-sec-Butyl-4,6-dinitrophenol

3.3"-Dichlorobenzidine

3,3"-Dimethylbenzidine

3-Methylcholanthrene

4-Aminobiphenyl

4-Bromophenyl phenyl ether

4-Chloro-rm-cresol

\author{
Screen Zone Elevation \\ $100.4-90.4 \mathrm{ft} \mathrm{msl}$
}

$02 / 28 / 00$

\begin{tabular}{l} 
18t Half \\
\hline 163.7 \\
4.4 \\
17 \\
18.3 \\
1 \\
.7 \\
3.25
\end{tabular}

1st Half
$<5$
$<5$
$<5$
$<5$
$<5$
$<5$
$<5$
$<5$
$<52$
$<11$
$<10$
$<5$
$<11$
$<5$
$<5$
$<10$
$<11$
$<5$
$<50$
$<11$
$<11$
$<11$
$<5$
$<11$
$<11$

\begin{tabular}{ll} 
CLP & EPA \\
\hline$J U$ & $L$ \\
$J U$ & $L$ \\
$J U$ & $L$ \\
$J U$ & $L$ \\
$J U$ & $L$ \\
$J U$ & $L$ \\
$J U$ & $L$ \\
$J U$ & $L$
\end{tabular}

Filt.

$<E Q L$

$<E Q L$

$<E Q L$

$<E Q L$

$<E Q L$

$<E Q L$

$<E Q L$

$<E Q L$

$<E Q L$

$<E Q L$

$<\mathrm{EQL}$

$<E Q L$

$<E Q L$

$<E Q L$

$<E Q L$

$<E Q L$

$<E Q L$

$<E Q L$

$<$ EQL

$<$ EQL

$<E Q L$

$<$ EQL

$<$ EQL

$<\mathrm{EQL}$

$<$ EQL

$<11 \quad U \quad<E Q L$

$<26 \quad U \quad<E Q L$

$<11 \quad U \quad<$ EQL

$<11 \quad U \quad<E Q L$

$<26 \quad U \quad<E Q L$

$<E Q L$

$<E Q L$

$<E Q L$

$<E Q L$

$<E Q L$

$<E Q L$

$L \quad<E Q L$

$<E Q L$

$<E Q L$

$<\mathrm{EQL}$

$<$ EQL

$<E Q L$

$<E Q L$

$<E Q L$

$<E Q L$

$<E Q L$

$<11 \quad U \quad<$ EQL $\quad$ ugh/L

Unit

ft msl

$\mathrm{pH}$

uS $/ \mathrm{cm}$

deg. C

$\mathrm{mg} / \mathrm{L}$

well volume creen Zone

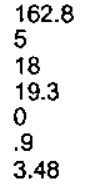

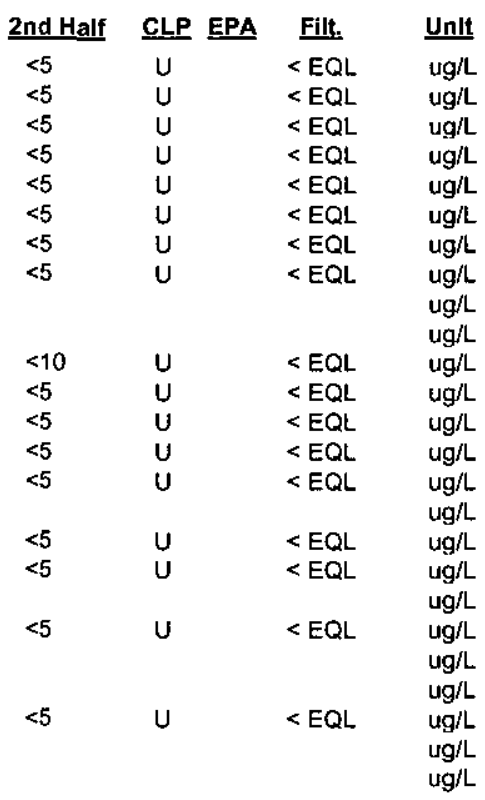

ug/L

ugh

ugh

ug/h

ug/L

$\mathrm{ug} / \mathrm{L}$

$\mathrm{ug} / \mathrm{L}$

ug/L

ug/L

$\mathrm{ug} / \mathrm{L}$

$<20 \quad \mathrm{U} \quad<\mathrm{EQL} \quad \mathrm{ug} / \mathrm{L}$

ug/L

ug/L

ug/

$\mathrm{ug} / \mathrm{L}$

ug/L

$\mathrm{ug} / \mathrm{L}$

ug/L

ug/

ug/L

Notes: Concentrations in bold italics exceed the groundwater protection standards listed in Appendix A. Bold italics were not assigned if the result was qualified with a "J", "R", "L", or "U" qualifier.

$+=$ exceeded the Groundwater Protection Standards listed in Appendix A. 
Table B. Assessment Monitoring Results for Groundwater Wells (Cont.)

WELL LFW 43B

ANALYTICAL DATA

Groundwater Protection Standard

ST Parameter

4-Chloroaniline

4-Chlorophenyl phenyl ether

4-Nitrophenol

5-Nitro-o-toluidine

7,12-Dimethylbenz(a)anthracene

Acenaphthene

Acenaphthylene

Acetone

Acetonitrile (Methyl cyanide)

Acetophenone

Acrolein

Acrylonitrile

Aldrin

Allyl chloride

Anthracene

Antimony, total recoverable

Arsenic, total recoverable

Barium, total recoverable

Benzene

Benzo(a)anthracene

Benzo(a)pyrene

Benzo(b)fluoranthene

Benzo(g,h,i)perylene

Benzo(k)fluoranthene

Benzyl alcohol

Beryllium, total recoverable

Bis(2-chloroethoxy) methane

Bis(2-chloroethyl) ether

Bis(2-ethylhexyl) phthalate

Bromochloromethane

Bromodichloromethane

Bromoform

Bromomethane (Methyl bromide)

Butylbenzyl phthalate

Cadmium, total recoverable

Carbon disulfide

Carbon tetrachloride

Chlorobenzene

Chlorobenzilate

Chloroethane

Chloroethene (Vinyl chloride)

Chloroform

Chloromethane (Methyl chloride)

Chloroprene

Chromium, total recoverable

Chrysene

Cobalt, total recoverable

Copper, total recoverable

Cyanide

Di-n-butyl phthalate

Di-n-octyl phthalate

Diallate

Dibenz $(\mathrm{a}, \mathrm{h})$ anthracene

Dibenzofuran

Dibromochloromethane

Dibromomethane (Methylene bromide)

Dichlorodifluoromethane

- Dichloromethane (Methylene chloride)

Dieldrin

Diethyl phthalate

Dimethoate

Dimethyl phthalate

Diphenylamine

Disulfoton

Endosulfan !

Endosulfan H!

Endosulfan sulfate

Endrin

\begin{tabular}{|c|c|c|c|}
\hline 1st Half & CLP & EPA & Filt. \\
\hline$<11$ & U & & $<$ EQL \\
\hline$<11$ & $U$ & & $<E Q L$ \\
\hline$<26$ & U & & $<$ EQL \\
\hline$<11$ & U & & $<$ EQL \\
\hline$<11$ & U & & $<$ EQL \\
\hline$<11$ & $\mathrm{U}$ & & $<E Q L$ \\
\hline$<11$ & U & & $<E Q L$ \\
\hline$<20$ & JU & $\mathbf{L}$ & $<E Q L$ \\
\hline$<200$ & JU & L & $<E Q L$ \\
\hline$<11$ & $\mathrm{U}$ & & $<E Q L$ \\
\hline$<50$ & JU & $\mathbf{L}$ & $<E Q L$ \\
\hline$<10$ & JU & $L$ & $<E Q L$ \\
\hline$<.1$ & $\mathrm{U}$ & & $<\mathrm{EQL}$ \\
\hline$<5$ & JU & L & $<\mathrm{EQL}$ \\
\hline$<11$ & $\mathrm{U}$ & & $<E Q L$ \\
\hline$<100$ & $\mathrm{U}$ & & $<E Q L$ \\
\hline$<5.01$ & JU & & $<E Q L$ \\
\hline 3.52 & $\mathrm{~J}$ & I & NDD \\
\hline$<5$ & JU & $\mathbf{L}$ & $<$ EQL \\
\hline$<11$ & $\mathrm{U}$ & & $<E Q L$ \\
\hline$<11$ & $\mathrm{U}$ & & $<E Q L$ \\
\hline$<11$ & $\mathrm{U}$ & & $<E Q L$ \\
\hline$<11$ & U & & $<\mathrm{EQL}$ \\
\hline$<11$ & $\mathrm{U}$ & & $<\mathrm{EQL}$ \\
\hline$<1$ & $U$ & & $<$ EQL \\
\hline$<11$ & U & & $<E Q L$ \\
\hline$<11$ & $U$ & & $<E Q L$ \\
\hline$<11$ & $\mathrm{U}$ & & $<\mathrm{EQL}$ \\
\hline$<5$ & JU & $\mathrm{L}$ & $<E Q L$ \\
\hline$<5$ & $\mathrm{JU}$ & $L$ & $<E Q L$ \\
\hline$<5$ & $\mathrm{JU}$ & $L$ & $<E Q L$ \\
\hline$<5$ & JU & $\mathrm{L}$ & $<E Q L$ \\
\hline$<11$ & $U$ & & $<E Q L$ \\
\hline$<10$ & U & & $<E Q L$ \\
\hline$<5$ & JU & $L$ & $<\mathrm{EQL}$ \\
\hline$<5$ & JU & $L$ & $<$ EQL \\
\hline$<5$ & JU & $\mathrm{L}$ & $<$ EQL \\
\hline$<11$ & $\mathrm{U}$ & & $<E Q L$ \\
\hline$<5$ & JU & $\mathrm{L}$ & $<E Q L$ \\
\hline$<5$ & JU & $\mathrm{L}$ & $<E Q L$ \\
\hline$<5$ & JU & $\mathrm{L}$ & $<E Q L$ \\
\hline$<5$ & JU & $L$ & $<\mathrm{EQL}$ \\
\hline$<20$ & JU & $\mathrm{L}$ & $<\mathrm{EQL}$ \\
\hline$<10$ & $\mathrm{U}$ & & $<E Q L$ \\
\hline$<11$ & $U$ & & $<$ EQL \\
\hline$<6.25$ & JU & V & $<E Q L$ \\
\hline \multirow[t]{2}{*}{$<2.83$} & JU & V & $<E Q L$ \\
\hline & $\cdot$ & & \\
\hline$<11$ & U & & $<\mathrm{EQL}$ \\
\hline$<11$ & U & & $<$ EQL \\
\hline$<11$ & U & & $<\mathrm{EQL}$ \\
\hline$<11$ & $U$ & & $<E Q L$ \\
\hline$<11$ & $U$ & & $<$ EQL \\
\hline$<5$ & JU & $L$ & $<\mathrm{EQL}$ \\
\hline$<5$ & JU & $L$ & $<\mathrm{EQL}$ \\
\hline$<5$ & JU & L & $<E Q L$ \\
\hline$<2.8$ & JU & LV & $<E Q L$ \\
\hline$<.21$ & $\mathbf{U}$ & & $<E Q L$ \\
\hline$<11$ & U & & $<E Q L$ \\
\hline$<11$ & U & & $<E Q L$ \\
\hline$<11$ & $U$ & & $<\mathrm{EQL}$ \\
\hline$<11$ & $U$ & & $<\mathrm{EQL}$ \\
\hline$<11$ & $\mathrm{U}$ & & $<\mathrm{EQL}$ \\
\hline$<.1$ & U & & $<$ EQL \\
\hline$<.21$ & $U$ & & $<E Q L$ \\
\hline$<.21$ & U & & $<E Q L$ \\
\hline$<.21$ & I & & $<F O t$ \\
\hline
\end{tabular}

$\begin{array}{ccc}\text { 2nd Half } & \text { CLP EPA } \\ & & \\ & & \\ & & \\ & & \\ & & <E Q L \\ <20 & U & <E Q L \\ <200 & U & <E Q L \\ <50 & U & <E Q L \\ <10 & U & <E Q L \\ <10 & U & <E Q L \\ & & <E Q L \\ <100 & U & <E Q L \\ 440 & U & \end{array}$

Unit

$\mathrm{ug} / \mathrm{t}$

$\operatorname{ug} / \mathrm{L}$

$\mathrm{ug} / \mathrm{L}$

$u g / L$

$\mathrm{ug} / \mathrm{L}$

ug/L

ug/L

$\mathrm{ug} / \mathrm{L}$

ug/L

$\mathrm{ug} / \mathrm{L}$

ug/L

$u g / L$

$\mathrm{ug} / \mathrm{L}$

ug/L

ug/L

ug/L

$\mathrm{ug} / \mathrm{L}$

$\mathrm{ug} / \mathrm{L}$

$u g / L$

ug/l

$\mathrm{ug} / \mathrm{L}$

$\mathrm{ug} / \mathrm{L}$

$<13 \quad \mathrm{JU} \quad<$ EQL ug/L

$\mathrm{ug} / \mathrm{L}$

$\mathrm{ug} / \mathrm{L}$

$<5 \quad U \quad<E Q L \quad \mathrm{ug} / \mathrm{L}$

$<5 \quad U<E Q L \quad u g / h$

$<5 \quad U \quad<E Q L \quad u g / L$

$<10 \quad U \quad<E Q L \quad \mathrm{ug} / \mathrm{h}$

$<10 \quad \mathrm{U} \quad<\mathrm{EQL} \quad \mathrm{ug} / \mathrm{L}$

$<5 \quad U \quad<E Q L \quad u g / L$

$<5 \quad U \quad<E Q L \quad u g / L$

$<5 \quad \mathrm{U} \quad<\mathrm{EQL} \quad \mathrm{ug} / \mathrm{L}$

$<10 \quad \mathrm{U} \quad<\mathrm{EQL} \quad \mathrm{ug} / \mathrm{L}$

$<10 \quad \mathrm{U} \quad<$ EQL Ug/L

$<5 \quad U \quad<E Q L \quad \mathrm{ug} / \mathrm{L}$

$<10 \quad \mathrm{U} \quad \mathrm{EQL} \quad \mathrm{ug} / \mathrm{L}$

$<20 \quad U \quad<E Q L \quad u g / L$

$<10 \quad \mathrm{E} \quad<\mathrm{EQ} \quad \mathrm{ug} / \mathrm{L}$

$<20 \quad<$ EQL $\quad$ ug/

$<20 \quad \mathrm{EQL} \quad \mathrm{ug} / \mathrm{L}$

$<9.5 \quad U \quad<E Q L \quad u g / L$

$\operatorname{ug} / \mathrm{h}$

$\mathrm{ug} / \mathrm{L}$

ug/L

ug/L

$<5 \quad \mathrm{U} \quad<\mathrm{EQL} \quad \mathrm{ug} / \mathrm{L}$

$<5 \quad U \quad<E Q L \quad u g / L$

$8.32>5 \mathrm{ug} / \mathrm{L}$

$\mathrm{ug} / \mathrm{L}$

$\mathrm{ug} / \mathrm{L}$

$\mathrm{ug} / \mathrm{L}$

$\mathrm{ug} / \mathrm{L}$

ug/L

ug/L

$\mathrm{ug} / \mathrm{L}$

$u g / L$

$\mathrm{ug} / \mathrm{L}$

$\mathrm{ug} / \mathrm{L}$

Notes: Concentrations in bold italics exceed the groundwater protection standards listed in Appendix A. Bold italics were not assigned if the result was qualified with a "J", "R", "L", or "U" qualifier.

$+=$ exceeded the Groundwater Protection Standards listed in Appendix A. 
Table B. Assessment Monitoring Results for Groundwater Wells (Cont.)

WELL LFW 43B

\section{ANALYTICAL DATA}

Groundwater Protection Standard

\section{ST Parameter}

Endrin aldehyde

Ethyl methacrylate

Ethyl methanesulfonate

Ethytbenzene

Famphur

Fluoranthene

Fluorene

Heptachlor

Heptachlor epoxide

Hexachlorobenzene

Hexachlorobutadiene

Hexachlorocyclopentadiene

Hexachloroethane

Hexachloropropene

Indeno(1,2,3-c,d)pyrene

lodomethane (Methyl iodide)

Isobutyl alcohol

Isodrin

Isophorone

Isosafrole

Kepone

Lead, total recoverable

Lindane

Mercury, total recoverable

Methacrylonitrile

Methapyrilene

Methoxychlor

Methyl ethyl ketone

Methyl isobutyl ketone

Methyl methacrylate

Methyl methanesulfonate

$\mathrm{N}$-Nitrosodi-n-butylamine

N-Nitrosodiethylamine

$\mathrm{N}$-Nitrosodimethylamine

$\mathrm{N}$-Nitrosodiphenylarnine

$\mathrm{N}$-Nitrosodipropylamine

N-Nitrosomethylethylamine

$\mathrm{N}$-Nitrosopiperidine

$\mathrm{N}$-Nitrosopyrrolidine

Naphthalene

Nickel, total recoverable

Nitrobenzene

O,O,O-Triethyl phosphorothioate

$\mathrm{PCB}$

PCB 1016

PCB 1232

PCB 1242

PCB 1248

PCB 1254

PCB 1260

Parathion

Parathion methyl

Pentachlorobenzene

Pentachforonitrobenzene

Pentachlorophenol

Phenacetin

Phenanthrene

Phenol

Phorate

Pronamid

Propionitrile

Pyrene

Safrole

Selenium, total recoverable

Silver, total recoverable

Specific conductance

Styrene

Sulfide

\begin{tabular}{|c|c|c|c|}
\hline Ist Half & CLP & EPA & Filt \\
\hline$<.21$ & U & & $<\mathrm{E}$ \\
\hline$<5$ & JU & L & \\
\hline$<11$ & U & & \\
\hline$<5$ & JU & $L$ & \\
\hline$<200$ & U & & $<\bar{E}$ \\
\hline$<11$ & U & & \\
\hline$<11$ & U & & $<B$ \\
\hline$<.1$ & u & & $<E$ \\
\hline$<.1$ & $u$ & & \\
\hline$<11$ & u & & $<$ \\
\hline$<11$ & u & & $<E$ \\
\hline$<11$ & U & & \\
\hline$<11$ & U & & \\
\hline$<52$ & U & & $<B$ \\
\hline$<11$ & U & & \\
\hline$<5$ & JU & L & \\
\hline$<500$ & JU & $\bar{L}$ & $<\mathrm{E}$ \\
\hline$<11$ & u & & $<$ \\
\hline$<11$ & u & & \\
\hline$<11$ & $u$ & & $<$ \\
\hline$<11$ & U & & \\
\hline$<10$ & U & & $<\mathrm{E}$ \\
\hline$<.1$ & U & & $<B$ \\
\hline$<.5$ & U & & \\
\hline$<200$ & JU & L & $<$ \\
\hline$<11$ & U & & \\
\hline$<1$ & $U$ & & \\
\hline$<20$ & $\mathrm{JU}$ & $\mathbf{L}$ & $<$ \\
\hline$<10$ & JU & L & $<$ \\
\hline$<20$ & JU & $\vec{L}$ & $<B$ \\
\hline$<11$ & U & & $<B$ \\
\hline$<11$ & $U$ & & \\
\hline$<11$ & $u$ & & $<B$ \\
\hline$<26$ & U & & $<E$ \\
\hline$<11$ & U & & \\
\hline$<11$ & $U$ & & \\
\hline$<11$ & U & & $<$ \\
\hline$<11$ & $U$ & & \\
\hline$<11$ & U & & \\
\hline$<11$ & U & & \\
\hline$<50$ & U & & \\
\hline$<11$ & U & & \\
\hline$<11$ & U & &  \\
\hline
\end{tabular}

$\begin{array}{ccc}\text { 2nd Half } & \text { CLP EPA } & \text { Filt. } \\ <5 & U & <E Q L \\ <5 & U & <E Q L\end{array}$

Unit

ug/h

$u g / L$

$\mathrm{ug} / \mathrm{L}$

$\mathrm{ug} / \mathrm{L}$

ug/h

$u g / L$

$\mathrm{ug} / \mathrm{L}$

ug/ $/$

ug/L

$\mathrm{ug} / \mathrm{L}$

$u g / L$

$u g / \mathrm{L}$

ug/

ug/L

$<5$

$<500$

$u g / L$

ug/L

ugl

ug/

ug/L

$u g / L$

$<47 \quad U \quad<E Q L \quad u g / L$

$<.5 \quad \mathrm{U} \quad<\mathrm{EQL} \quad \mathrm{ug} / \mathrm{L}$

$<200 \quad \mathrm{~L}<\mathrm{EQL} \quad \mathrm{ug} / \mathrm{L}$

$\mathrm{ug} / \mathrm{L}$

$\mathrm{ug} / \mathrm{L}$

$\begin{array}{llll}<20 & U & <E Q L & \mathrm{ug} / L \\ <10 & U & <E Q L & \mathrm{ug} / \mathrm{L}\end{array}$

$<20 \quad \mathrm{U} \quad<\mathrm{EQL} \quad \mathrm{ug} / \mathrm{L}$

$\mathrm{ug} / \mathrm{L}$

$u g / L$

ug/L

ug/L

$u g / L$

$u g / L$

ug/L

$u g / L$

$\mathrm{ug} / \mathrm{L}$

$\mathrm{ug} / \mathrm{L}$

$<50 \quad U \quad<$ EQL $\quad u g / L$

$\operatorname{ug} / 2$

$\mathrm{ug} / \mathrm{L}$

$\begin{array}{llll}<11 & U & <E Q L \\ <11 & U & <E Q L \\ <52 & U & <E Q L \\ <11 & U & <E Q L \\ <26 & U & <E Q L \\ <11 & U & <\text { EQL } \\ <11 & U & <E Q L \\ <11 & U & <E Q L \\ <11 & U & <E Q L \\ <11 & U & <E Q L \\ <200 & J U & L & <E Q L \\ <11 & U & & <E Q L \\ <11 & U & <E Q L \\ <10 & U & <E Q L \\ <20 & U & <E Q L \\ 16.1 & & <37 \\ <5 & J U & L & <E Q L\end{array}$

$<$ EQL

$<E Q L$

$<E Q L$

EQL

EQL

$<E Q L$

$<E Q L$

$<E Q L$

$<\mathrm{EQL}$

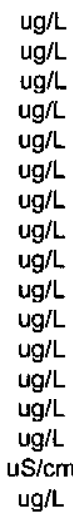

$\mathrm{ug} / \mathrm{L}$

Notes: Concentrations in bold italics exceed the groundwater protection standards listed in Appendix A. Bold italics were not assigned if the result was qualified with a "J", "R", "L", or "U" qualifier.

$+=$ exceeded the Groundwater Protection Standards listed in Appendix A. 
Table B. Assessment Monitoring Results for Groundwater Wells (Cont.)

\section{WELL LFW 43B}

ANALYTICAL DATA

Groundwater Protection Standard

\section{ST Parameter}

Tetrachloroethylene

Thallium, total recoverable

Thionazin

Tin, total recoverable

Toluene

Toxaphene

Trichloroethylene

Trichlorofluoromethane

Vanadium, total recoverable

Vinyl acetate

Xylenes

Zinc, total recoverable

alpha-Benzene hexachloride alpha-Chlordane

beta-Benzene hexachloride

cis-1,2-Dichloroethylene

cis-1,3-Dichloropropene

delta-Benzene hexachloride

gamma-Chlordane

m-Cresol (3-Methytphenol)

m-Nitroartiline

o-Cresol (2-Methylphenol)

o-Nitroaniline

o-Toluidine

p.p"-DDD

$p, p^{\prime \prime}-D D E$

p,p"-DDT

p-Cresol (4-Methylphenol)

p-Dimethylaminoazobenzene

p-Nitroaniline

p-Phenylenediamine

$\mathrm{pH}$

trans-1,2-Dichloroethylene

trans-1,3-Dichloropropene

trans-1,4-Dichloro-2-butene

\begin{tabular}{|c|c|c|c|}
\hline 1st Half & CLP & EPA & Filit. \\
\hline$<5$ & $\mathrm{JU}$ & L & $<E Q L$ \\
\hline$<10$ & U & & $<$ EQL \\
\hline$<11$ & $U$ & & $<E Q L$ \\
\hline$<200$ & U & & $<E Q L$ \\
\hline$<5$ & JU & $L$ & $<\mathrm{EQL}$ \\
\hline$<2.1$ & $\mathrm{U}$ & & $<E Q L$ \\
\hline$<5$ & JU & L & $<E Q L$ \\
\hline$<5$ & JU & $L$ & $<E Q L$ \\
\hline$<10$ & $U$ & & $<\mathrm{EQL}$ \\
\hline$<5$ & JU & $L$ & $<E Q L$ \\
\hline$<10$ & JU & L & $<\mathrm{EQL}$ \\
\hline 10.2 & $J$ & 1 & NDD \\
\hline$<.1$ & U & & $<E Q L$ \\
\hline$<.1$ & U & & $<$ EQL \\
\hline$<.1$ & $\mathbf{U}$ & & $<E Q L$ \\
\hline$<5$ & JU & L & $<E Q L$ \\
\hline$<5$ & JU & $L$ & $<\mathrm{EQL}$ \\
\hline$<.1$ & $U$ & & $<\mathrm{EQL}$ \\
\hline$<.1$ & $u$ & & $<$ EQL \\
\hline$<26$ & U & & $<$ EQL \\
\hline$<11$ & U & & $<E Q L$ \\
\hline$<26$ & $U$ & & $<\mathrm{EQL}$ \\
\hline$<11$ & $U$ & & $<\mathrm{EQL}$ \\
\hline$<.21$ & $U$ & & $<\mathrm{EQL}$ \\
\hline$<.21$ & U & & $<\mathrm{EQL}$ \\
\hline$<.21$ & U & & $<E Q L$ \\
\hline$<10$ & $U$ & & $<E Q L$ \\
\hline$<11$ & U & & $<$ EQL \\
\hline$<11$ & U & & $<E Q L$ \\
\hline$<11$ & U & & $<E Q L$ \\
\hline 5.64 & $J$ & $Q$ & NDD \\
\hline$<5$ & JU & $\mathbf{L}$ & $<\mathrm{EQL}$ \\
\hline$<5$ & JU & $\mathbf{L}$ & $<E Q L$ \\
\hline$<20$ & JU & L & $<$ EQL \\
\hline
\end{tabular}

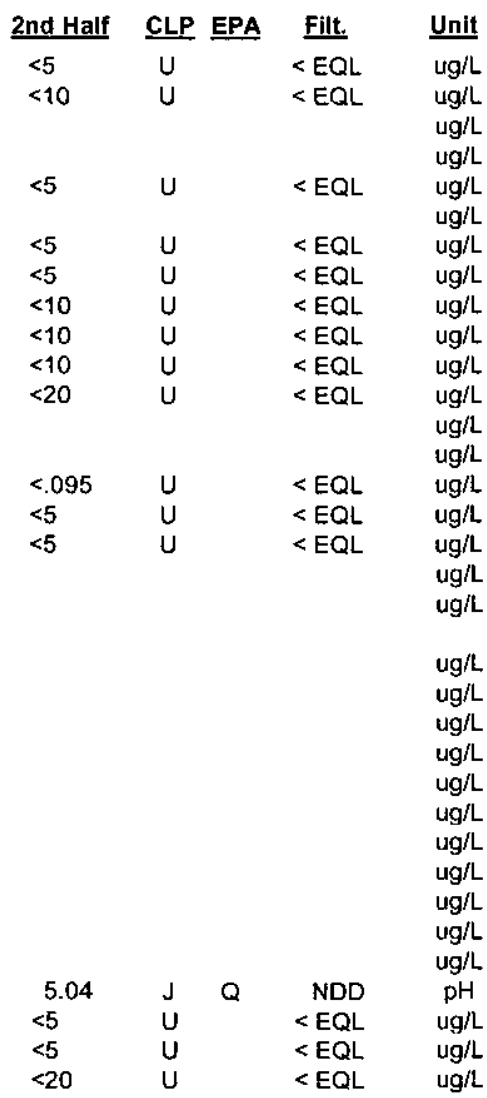

Notes: Concentrations in bold italics exceed the groundwater protection standards listed in Appendix A. Bold italics were not assigned if the result was qualified with a "J", "R", "L", or "U" qualifier.

$+=$ exceeded the Groundwater Protection Standards Iisted in Appendix A. 
Table B. Assessment Monitoring Results for Groundwater Wells (Cont.)

WELL LFW 43C

$\begin{array}{ll}\text { SRS Coord. } & \text { Lat/Longitude } \\ \text { N } 86480.6 & \text { 33.29084 Deg N } \\ \text { E 45234.9 } & -81.7157 \mathrm{Deg} W\end{array}$

SAMPLE DATE

\section{FIELD DATA}

\section{Parameter}

Water Elevation

$\mathrm{pH}$

Sp. Conductance

Water temperature

Alkalinity as $\mathrm{CaCO} 3$

Turbidity

Volumes purged

Sampling code

\section{ANALYTICAL DATA}

Groundwater Protection Standard

\section{ST Parameter}

1,1,1,2-Tetrachloroethane

1,1,1-Trichloroethane

$1,1,2,2$-Tetrachloroethane

1,1,2-Trichloroethane

1,1-Dichloroethane

1,1-Dichloroethylene

1,1-Dichloropropene

1,2,3-Trichloropropane

1,2,4,5-Tetrachlorobenzene

1,2,4-Trichlorobenzene

1,2-Dibromo-3-chloropropane

1,2-Dibromoethane

1,2-Dichlorobenzene

1,2-Dichloroethane

1,2-Dichloropropane

1,3,5-Trinitrobenzene

1,3-Dichlorobenzene

1,3-Dichloropropane

1,3-Dinitrobenzene

1,4-Dichlorobenzene

1,4-Naphthoquinone

1-Naphthylamine

2,2-Dichloropropane

2,2-Oxybis (1-chloropropane)

2,3,4,6-Tetrachlorophenol

$2,4,5-T$

2,4,5-TP (Silvex)

2,4,5-Trichlorophenol

2,4,6-Trichlorophenol

2,4-Dichlorophenol

2,4-Dichlorophenoxyacetic acid

2,4-Dimethyl phenol

2,4-Dinitrophenol

2,4-Dinitrotoluene

2,6-Dichloropheno

2,6-Dinitrotoluene

2-Acetylaminofluorene

2-Chloronaphthalene

2-Chlorophenol

2-Hexanone

2-Methyl-4,6-dinitrophenol

2-Methylnaphthalene

2-Naphthylamine

2-Nitrophenol

2-sec-Butyl-4,6-dinitrophenol

$3,3^{\prime \prime}$-Dichlorobenzidine

3,3"-Dimethylbenzidine

3-Methylcholanthrene

4-Aminobiphenyl

4-Bromophenyl phenyl ether

4-Chloro-m-cresol

\author{
Screen Zone Elevation \\ $138.5-128.5 \mathrm{ft} \mathrm{ms}$
}

$02 / 28 / 00$

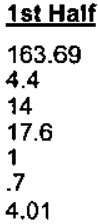

\begin{tabular}{l} 
1st Half \\
\hline$<5$ \\
$<5$ \\
$<5$ \\
$<5$ \\
$<5$ \\
$<5$ \\
$<5$ \\
$<5$ \\
$<52$ \\
$<11$ \\
$<10$ \\
$<5$ \\
$<11$ \\
$<5$ \\
$<5$ \\
$<10$ \\
$<11$ \\
$<5$ \\
$<50$ \\
$<11$ \\
$<11$ \\
$<11$ \\
$<5$ \\
$<11$ \\
$<11$
\end{tabular}

Filt.

$<$ EQL

$<E Q L$

$<E Q L$

$<E Q L$

$<E Q L$

$<E Q L$

$<E Q L$

$<E Q L$

$<E Q L$

$<E Q L$

$<E Q L$

$<$ EQL

$<E Q L$

$<E Q L$

$<E Q L$

$<E Q L$

$<E Q L$

$<E Q L$

$<E Q L$

$<$ EQL

$<E Q L$

$<E Q L$

$<E Q L$

$<$ EQL

$<$ EQL

$<11 \quad U \quad<E Q L$

$<26$

$<11$

$<11$

$<26$

$<11$

$<25$

$<11$

$<11$

$<11$

$<11$

$<20$

$<26$

$<11$

$<11$

$<11$

$<11$

$<21$

$<11$

$<11$

$<11$

$<11$

$<\mathrm{EQL}$

$<E Q L$

$<E Q L$

$<$ EQL

$<E Q L$

$<E Q L$

$<$ EQL

$<$ EQL

$<$ EQL

$<$ EQL

$<\mathrm{EQL}$

$<E Q L$

$<E Q L$

$<$ EQL

$<$ EQL

$<$ EQL

2nd Half
$\mathbf{1 6 2 . 8 3}$
5.2
15
19.3
0
1.3
4.78

Unit

ft ms!

$\mathrm{pH}$

uS $/ \mathrm{cm}$

deg. C

$\mathrm{mgl} / \mathrm{L}$

well volume
Screen Zone

Upper

Notes: Concentrations in bold italics exceed the groundwater protection standards listed in Appendix A. Bold italics were not assigned if the result was qualified with a "J", "R", "L", or "U" qualifier.

$+=$ exceeded the Groundwater Protection Standards listed in Appendix A.

$\mathrm{ug} / \mathrm{L}$

$\mathrm{ug} / \mathrm{L}$ 
Table B. Assessment Monitoring Results for Groundwater Wells (Cont.)

WELL LFW 43C

\section{ANALYTICAL DATA}

Groundwater Protection Standard

ST Parameter

4-Chloroaniline

4-Chlorophenyl phenyl ether

4-Nitrophenol

5-Nitro-o-toluidine

7,12-Dimethylbenz(a)anthracene

Acenaphthene

Acenaphthylene

Acetone

Acetonitrile (Methyl cyanide)

Acetophenone

Acrolein

Acrylonitrile

Aldrin

Allyl chloride

Anthracene

Antimony, total recoverable

Arsenic, total recoverable

Barium, total recoverable

Benzene

Benzo(a)anthracene

Benzo(a)pyrene

Benzo(b)fluoranthene

Benzo(g,h,i)perylene

Benzo(k)fluoranthene

Benzyl alcohol

Beryllium, total recoverable

Bis(2-chloroethoxy) methane

Bis(2-chloroethyl) ether

Bis(2-ethylhexyl) phthalate

Bromochloromethane

Bromodichloromethane

Bromoform

Bromomethane (Methyl bromide)

Butylbenzyl phthalate

Cadmium, total recoverable

Carbon disulfide

Carbon tetrachloride

Chlorobenzene

Chlorobenzilate

Chloroethane

Chloroethene (Vinyl chloride)

Chloroform

Chloromethane (Methyl chloride)

Chloroprene

Chromium, total recoverable

Chrysene

Cobalt, total recoverable

Copper, total recoverable

Cyanide

Di-n-butyl phthalate

Di-n-octyl phthalate

Diallate

Dibenz(a,h)anthracene

Dibenzofuran

Dibromochloromethane

Dibromomethane (Methylene bromide)

Dichlorodiffuoromethane

Dichloromethane (Methylene chloride)

Dieldrin

Diethyl phthaiate

Dimethoate

Dimethyl phthalate

Diphenylarnine

Disulfoton

Endosulfan I

Endosulfan II

Endosulfan sulfate

Endrin

\begin{tabular}{|c|c|c|}
\hline 1st Haif & $\underline{\text { CLP }}$ & EPA \\
\hline$<11$ & $\mathrm{U}$ & \\
\hline$<11$ & $U$ & \\
\hline$<26$ & U & \\
\hline$<11$ & $\mathrm{U}$ & \\
\hline$<11$ & $U$ & \\
\hline$<11$ & U & \\
\hline$<11$ & U & \\
\hline$<20$ & U & \\
\hline$<200$ & U & \\
\hline$<11$ & U & \\
\hline$<50$ & $U$ & \\
\hline$<10$ & U & \\
\hline$<.1$ & $U$ & \\
\hline$<5$ & $\mathrm{U}$ & \\
\hline$<11$ & $U$ & \\
\hline$<100$ & $\mathrm{U}$ & \\
\hline$<10$ & $U$ & \\
\hline 2.99 & \rfloor & 1 \\
\hline$<5$ & $U$ & \\
\hline$<11$ & U & \\
\hline$<11$ & $U$ & \\
\hline$<11$ & $\bigcup$ & \\
\hline$<11$ & U & \\
\hline$<11$ & $\mathrm{U}$ & \\
\hline$<1$ & $\mathrm{U}$ & \\
\hline$<11$ & $U$ & \\
\hline$<11$ & U & \\
\hline 6.6 & $\mathrm{~J}$ & 1 \\
\hline$<5$ & $U$ & \\
\hline$<5$ & $U$ & \\
\hline$<5$ & U & \\
\hline$<5$ & $\mathbf{U}$ & \\
\hline$<11$ & $\mathrm{U}$ & \\
\hline$<10$ & $U$ & \\
\hline$<5$ & $\mathrm{U}$ & \\
\hline$<5$ & $\mathrm{U}$ & \\
\hline$<5$ & U & \\
\hline$<11$ & U & \\
\hline$<5$ & U & \\
\hline$<5$ & $U$ & \\
\hline$<5$ & U & \\
\hline$<5$ & $U$ & \\
\hline$<20$ & $U$ & \\
\hline$<10$ & U & \\
\hline$<11$ & $U$ & \\
\hline$<5.19$ & JU & V \\
\hline$<3.96$ & JU & \\
\hline$<11$ & U & \\
\hline$<11$ & U & \\
\hline$<11$ & U & \\
\hline$<11$ & $\mathrm{U}$ & \\
\hline$<11$ & $\mathrm{U}$ & \\
\hline$<5$ & U & \\
\hline$<5$ & U & \\
\hline$<5$ & $U$ & \\
\hline$<10$ & U & \\
\hline$<.21$ & U & \\
\hline$<11$ & $U$ & \\
\hline$<11$ & U & \\
\hline$<11$ & U & \\
\hline$<11$ & U & \\
\hline$<11$ & $U$ & \\
\hline$<.1$ & U & \\
\hline$<.21$ & $U$ & \\
\hline$<.21$ & $U$ & \\
\hline$<.21$ & $\mathrm{U}$ & \\
\hline
\end{tabular}

Filt.

EQL

$<E Q L$

$<E Q L$

$<E Q L$

$<$ EQL

$<$ EQL

$<E Q L$

$<E Q L$

$<E Q L$

$<\mathrm{EQL}$

$<E Q L$

$<$ EQL

$<$ EQL

$<\mathrm{EQL}$

$<E Q L$

$<$ EQL

$<E Q L$

NDD

$<$ EQL

$<$ EQL

$<E Q L$

$<E Q L$

$<E Q L$

$<E Q L$

$<$ EQL

$<$ EQL

$<$ EQL

NDD

$<E Q L$

$<$ EQL

$<$ EQL

$<E Q L$

$<E Q L$

$<$ EQL

$<E Q L$

$<E Q L$

$<E Q L$

$<$ EQL

$<$ EQL

$<E Q L$

$<E Q L$

$<E Q L$

$<E Q L$

$<$ EQL

$<E Q L$

$<$ EQL

$<$ EQL

$<E Q L$

$<$ EQL

$<$ EQL

$<E Q L$

$<$ EQL

$<E Q L$

$<$ EQL

$<E Q L$

$<E Q L$

$<E Q L$

$<E Q L$

$<$ EQL

$<E Q L$

$<$ EQL

$<E Q L$

$<E Q L$

$<E Q L$

$<E Q L$ 2nd Half CLP EPA Filt.

Unit

ugh

$\mathrm{ug} / \mathrm{L}$

ug/L

ug/L

$\mathrm{ug} / \mathrm{L}$

ug/L

$<20 . \quad U \quad<E Q L \quad u g / L$

$<200 \quad \mathrm{U} \quad<$ EQL Ug/L

$<50 \quad U \quad<E Q L \quad \mathrm{ug} / \mathrm{L}$

$<10 \quad U \quad<E Q L \quad u g / L$

$<5 \quad U \quad<E Q L \quad u g / L$

4.48 J I NDD $\quad$ ug/L

3.42 J I NDD ug/L

$<5 \quad U \quad<E Q L \quad u g / L$

$\mathrm{ug} / \mathrm{L}$

$u g / L$

ug/L

$\mathrm{ug} / \mathrm{L}$

$\mathrm{ug} / \mathrm{L}$

$<13 \quad \mathrm{JU} \quad<\mathrm{EQL}$

$u g / L$

$\mathrm{ug} / \mathrm{L}$

ug/

$u g / L$

$\begin{array}{llll}<5 & \mathrm{U} & <\mathrm{EQL} & \mathrm{ug} / \mathrm{L} \\ <5 & \mathrm{U} & <\mathrm{EQL} & \mathrm{ug} / \mathrm{L}\end{array}$

$<5 \quad U \quad<E Q L \quad u g / L$

$<5 \quad U \quad<E Q L \quad u g / L$

$<10 \quad U \quad<\mathrm{EQL} \quad \mathrm{ug} / \mathrm{L}$

$<5 \quad U \quad<E Q L \quad u g / L$

$<5 \quad U<\mathrm{EQL} \quad \mathrm{ug} / \mathrm{L}$

$<5 \quad U<\mathrm{EQ} \quad \mathrm{ug} / \mathrm{L}$

$\mathrm{ug} / \mathrm{L}$

$\begin{array}{llll}<5 & \mathrm{U} & <\mathrm{EQL} & \mathrm{ug} / \mathrm{L} \\ <5 & \mathrm{U} & <\mathrm{EQL} & \mathrm{ug} / \mathrm{L}\end{array}$

$<5 \quad \mathrm{U}<\mathrm{EQL} \quad \mathrm{ug} / \mathrm{L}$

$<5 \quad \mathrm{U}<\mathrm{EQL} \quad \mathrm{ug} / \mathrm{L}$

$<20 \quad U<\mathrm{EQL} \quad \mathrm{ug} / \mathrm{L}$

$<10 \quad \mathrm{U}<\mathrm{EQL} \mathrm{ug} / \mathrm{L}$

40 U $<$ EQL

$<20 \quad U \quad<E Q L \quad u g / L$

$<9.5 \quad U \quad<E Q L \quad u g / L$

$\mathrm{ug} / \mathrm{L}$

$u g / L$

$\mathrm{ug} / \mathrm{L}$

$u g / L$

$<5 \quad U<\mathrm{EQL} \quad \mathrm{Ug} / \mathrm{L}$

$<5 \quad U<\mathrm{UQL} \quad \mathrm{ug} / \mathrm{L}$

$<5 \quad U<\mathrm{EQL} \quad \mathrm{ug} / \mathrm{L}$

$<10 \quad \mathrm{U}<\mathrm{EQL} \quad \mathrm{ug} / \mathrm{L}$

$\mathrm{ug} / \mathrm{L}$

$\mathrm{ug} / \mathrm{L}$

$\mathrm{ug} / \mathrm{L}$

ugh

$\mathrm{ug} / \mathrm{L}$

$\mathrm{ug} / \mathrm{L}$

$\mathrm{ug} / \mathrm{L}$

ug/L

ug/L

Notes: Concentrations in bold italics exceed the groundwater protection standards listed in Appendix A. Bold italics were not assigned if the result was qualified with a "J", "R", "L", or "U" qualifier.

$+=$ exceeded the Groundwater Protection Standards listed in Appendix A. 


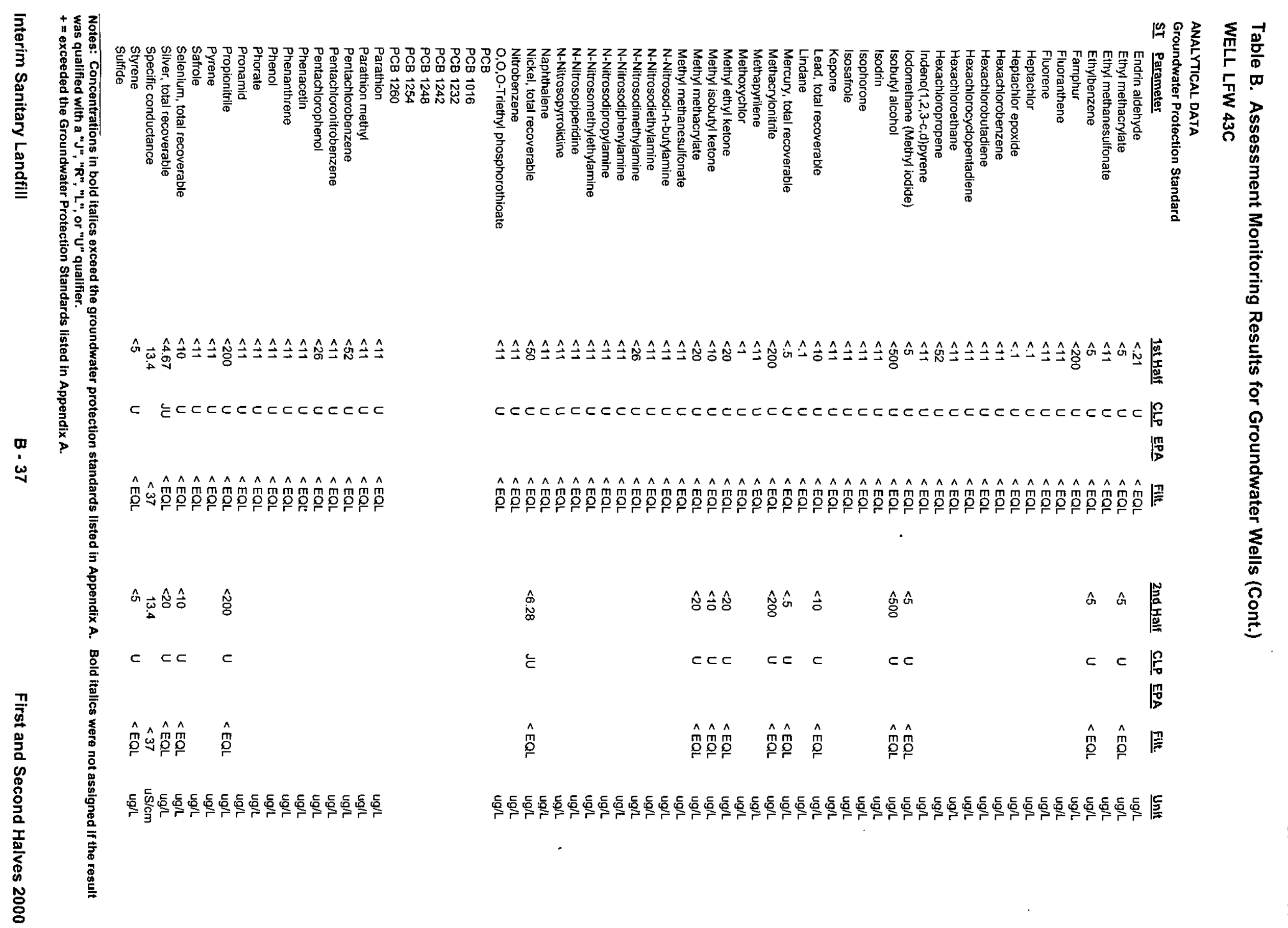

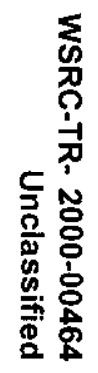


Table B. Assessment Monitoring Results for Groundwater Wells (Cont.)

WELL LFW 43C

ANALYTICAL DATA

Groundwater Protection Standard

\section{ST Parameter}

Tetrachloroethylene

Thallium, total recoverable

Thionazin

Tin, total recoverable

Toluene

Toxaphene

Trichloroethylene

Trichlorofluoromethane

Vanadium, total recoverable

Vinyl acetate

Xylenes

Zinc, total recoverable

aipha-Benzene hexachloride

alpha-Chlordane

beta-Benzene hexachloride

cis-1,2-Dichloroethylene

cis-1,3-Dichloropropene

delta-Benzene hexachloride

gamma-Chlordane

m-Cresol (3-Methylphenol)

m-Nitroaniline

o-Cresol (2-Methylphenol)

o-Nitroaniline

o-Toluidine

$p, p^{\prime \prime}-$ DDD

p, p"-DDE

$p, p^{\prime \prime}-D D T$

p-Cresol (4-Methylphenol)

p-Dimethylaminoazobenzene

p-Nitroaniline

p-Phenylenediamine

$\mathrm{pH}$

trans-1,2-Dichloroethylene

trans-1,3-Dichloropropene

trans-1,4-Dichloro-2-butene

\begin{tabular}{|c|c|c|c|}
\hline st Half & CLP & EPA & Filt. \\
\hline$<5$ & U & & $<E Q L$ \\
\hline$<7.36$ & JU & & $<\mathrm{EQL}$ \\
\hline$<11$ & U & & $<E Q L$ \\
\hline$<200$ & $\mathrm{U}$ & & $<E Q L$ \\
\hline$<5$ & U & & $<E Q L$ \\
\hline$<2.1$ & U & & $<E Q L$ \\
\hline$<5$ & $\mathrm{U}$ & & $<\mathrm{EQL}$ \\
\hline$<5$ & $U$ & & $<E Q L$ \\
\hline$<10$ & $U$ & & $<\mathrm{EQL}$ \\
\hline$<5$ & $\mathrm{U}$ & & $<$ EQL \\
\hline$<10$ & $\mathrm{U}$ & & $<E Q L$ \\
\hline$<20$ & U & & $<E Q L$ \\
\hline$<.1$ & U & & $<E Q L$ \\
\hline$<.1$ & U & & $<$ EQL \\
\hline$<.1$ & $U$ & & $<E Q L$ \\
\hline$<5$ & $U$ & & $<\mathrm{EQL}$ \\
\hline$<5$ & U & & $<E Q L$ \\
\hline$<.1$ & $U$ & & $<E Q L$ \\
\hline$<.1$ & U & & $<E Q L$ \\
\hline$<26$ & U & & $<E Q L$ \\
\hline$<11$ & U & & $<E Q L$ \\
\hline$<26$ & $U$ & & $<\mathrm{EQL}$ \\
\hline$<11$ & $U$ & & $<\mathrm{EQL}$ \\
\hline$<.21$ & $\mathrm{U}$ & & $<E Q L$ \\
\hline$<.21$ & $U$ & & $<\mathrm{EQL}$ \\
\hline$<.21$ & $\mathbf{U}$ & & $<\mathrm{EQL}$ \\
\hline$<10$ & $U$ & & $<E Q L$ \\
\hline$<11$ & $\mathbf{U}$ & & $<E Q L$ \\
\hline$<11$ & $U$ & & $<E Q L$ \\
\hline$<11$ & $U$ & & $<\mathrm{EQL}$ \\
\hline 5.72 & $J$ & $Q$ & NDL \\
\hline$<5$ & U & & $<E Q L$ \\
\hline$<5$ & U & & $<\mathrm{EQ}$ \\
\hline$<20$ & U & & $<\mathrm{EQ}$ \\
\hline
\end{tabular}

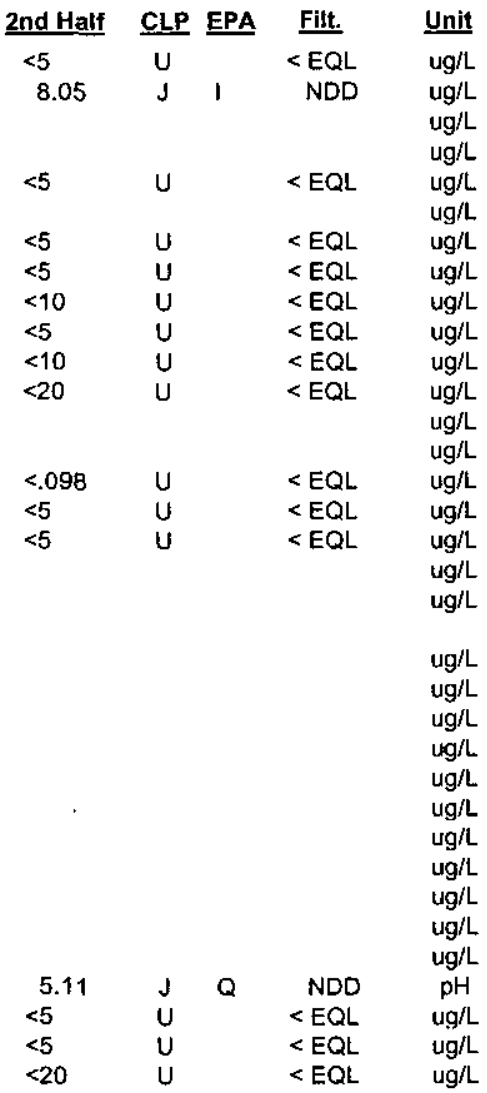

Notes: Concentrations in bold italics exceed the groundwater protection standards listed in Appendix A. Bold italics were not assigned if the result was qualified with a "J", "R", "L", or "U" qualifier.

$+=$ exceeded the Groundwater Protection Standards listed in Appendix A 
Table B. Assessment Monitoring Results for Groundwater Wells (Cont.)

WELL LFW 43D

\begin{tabular}{|c|c|}
\hline SRS Coord. & Lat/Longitude \\
\hline $\begin{array}{l}N 864 \\
E 452\end{array}$ & $\begin{array}{l}33.29077 \mathrm{D} \\
-81.7156 \mathrm{D}\end{array}$ \\
\hline
\end{tabular}

SAMPLE DATE

\section{FIELD DATA}

Parameter

\section{Water Elevation}

$\mathrm{pH}$

Sp. Conductance

Water temperature

Alkalinity as $\mathrm{CaCO} 3$

Turbidity

Volumes purged

Sampling code

\section{ANALYTICAL DATA}

Groundwater Protection Standard ST Parameter

$1,1,1,2-$ Tetrachloroethane

$1,1,1$-Trichloroethane

1,1,2,2-Tetrachloroethane

1,1,2-Trichloroethane

1,1-Dichloroethane

1,1-Dichloroethylene

1,1-Dichloropropene

1,2,3-Trichloropropane

1,2,4,5-Tetrachlorobenzene

1,2,4-Trichlorobenzene

1,2-Dibromo-3-chloropropane

1,2-Dibromoethane

1,2-Dichlorobenzene

1,2-Dichloroethane

1,2-Dichloropropane

1,3,5-Trinitrobenzene

1,3-Dichlorobenzene

1,3-Dichloropropane

1,3-Dinitrobenzene

1,4-Dichlorobenzene

1,4-Naphthoquinone

1-Naphthylamine

2,2-Dichloropropane

2,2-Oxybis (1-chloropropane)

2,3,4,6-Tetrachlorophenol

2,4,5-T

2,4,5-TP (Silvex)

2,4,5-Trichlorophenol

2,4,6-Trichlorophenol

2,4-Dichlorophenol

2,4-Dichlorophenoxyacetic acid

2,4-Dimethyl phenol

2,4-Dinitrophenol

2,4-Dinitrotoluene

2,6-Dichlorophenol

2,6-Dinitrotoluene

2-Acetylaminofluorene

2-Chloronaphthalene

2-Chlorophenol

2-Hexanone

2-Methyl-4,6-dinitrophenol

2-Methylnaphthalene

2-Naphthylamine

2-Nitrophenol

2-sec-Butyl-4,6-dinitrophenol

3,3"-Dichlorobenzidine

3,3"-Dimethylbenzidine

3-Methylcholanthrene

4-Aminobiphenyl

4-Bromophenyl phenyl ether

4-Chloro-m-cresol

\author{
Screen Zone Elevation \\ $170.9-150.9 \mathrm{ft} \mathrm{ms}$
}

$02 / 28 / 00$

1st Half
163.8
5
11
17.8
3
1.2
6.12

\begin{tabular}{l} 
1st Half \\
\hline$<5$ \\
$<5$ \\
$<5$ \\
$<5$ \\
$<5$ \\
$<5$ \\
$<5$ \\
$<5$ \\
$<52$ \\
$<11$ \\
$<10$ \\
$<5$ \\
$<11$ \\
$<5$ \\
$<5$ \\
$<10$ \\
$<11$ \\
$<5$ \\
$<50$ \\
$<11$ \\
$<11$ \\
$<11$ \\
$<5$ \\
$<11$ \\
$<11$ \\
$<5$
\end{tabular}

$<11$

$<26$

$<11$

$<11$

$<26$

$<11$

$<11$

$<11$

$<11$

$<11$

$<20$

$<26$

$<11$

$<11$

$<11$

$<11$

$<21$

$<11$

$<11$

$<11$

$<11$

\author{
Top of Casing \\ $202.9 \mathrm{ft} \mathrm{ms!}$
}

$\frac{\text { Casing }}{4 \text { "PVC }}$

Pump

Screen Zone

$10 / 02 / 00$

$\begin{array}{ll}\text { 2nd Half } & \text { Unit } \\ 162.88 & \text { ft msl } \\ 5.2 & \mathrm{pH} \\ 11 & \text { uS/cm } \\ 18.8 & \text { deg. C } \\ 0 & \mathrm{mg} / \mathrm{L} \\ 1.3 & \mathrm{NTU} \\ 4.01 & \text { well volume }\end{array}$

Upper

Notes: Concentrations in bold italics exceed the groundwater protection standards listed in Appendix A. Bold Italics were not assigned if the result was qualified with a "J", "R", "L", or "U" qualifier.

$+=$ exceeded the Groundwater Protection Standards listed in Appendix A. 
Table B. Assessment Monitoring Results for Groundwater Wells (Cont.)

WELL LFW 430

ANALYTICAL DATA

Groundwater Protection Standard

ST Parameter

4-Chloroanitine

4-Chlorophenyl phenyl ether

4-Nitrophenol

5-Nitro-o-toluidine

7,12-Dimethylbenz(a)anthracene

Acenaphthene

Acenaphthylene

Acetone

Acetonitrile (Methyl cyanide)

Acetophenone

Acrolein

Acrylonitrile

Aldrin

Allyl chloride

Anthracene

Antimony, total recoverable

Arsenic, total recoverable

Barium, total recoverable

Benzene

Benzo(a)anthracene

Benzo(a)pyrene

Benzo(b)fluoranthene

Benzo(g,h,i)perylene

Benzo(k)fluoranthene

Benzyl alcohol

Beryllium, total recoverable

Bis(2-chloroethoxy) methane

Bis(2-chloroethyl) ether

Bis(2-ethylhexyl) phthalate

Bromochloromethane

Bromodichloromethane

Bromoform

Bromomethane (Methyl bromíde)

Butylbenzyl phthalate

Cadmium, total recoverable

Carbon disulfide

Carbon tetrachloride

Chlorobenzene

Chlorobenzilate

Chlorosthane

Chloroethene (Vinyl chloride)

Chloroform

Chloromethane (Methyl chloride)

Chloroprene

Chromium, total recoverable

Chrysene

Cobalt, total recoverable

Copper, total recoverable

Cyanide

Di-n-butyl phthatate

Di-n-octyl phthalate

Diallate

Dibenz(a,h)anthracene

Dibenzofuran

Dibromochloromethane

Dibromomethane (Methylene bromide)

Dichlorodifluoromethane

Dichloromethane (Methylene chloride)

Dieldrin

Diethyl phthalate

Dimethoate

Dimethyl phthalate

Diphenylamine

Disulfoton

Endosulfan I

Endosulfan II

Endosulfan sulfate

Endrin

1st

$<11 \quad U$ <EQ

$<11 \quad U$

$<26 \quad U \quad<E Q L$

$<11 \quad U<E Q$

$<11 \quad U \quad<E Q L$

$<11 \quad U<E Q L$

$<11 \quad U<$ <

$<20 \quad U<E Q L$

$<200 \quad U<E Q L$

$<11 \quad U \quad<E Q L$

$<50 \quad U<E Q L$

$<10 \quad U<E Q L$

$<1 \quad U<E Q$

$<5 \quad U \quad<E Q L$

$<11 \quad U<E Q$

$<100 \quad U \quad<E Q L$

$<10 \quad U<$ EQL

6.65 J I NDD

$<5 \quad U \quad<E Q L$

$<11 \quad U<E Q L$

$<11 \quad U \quad<E Q L$

$<11 \quad U \quad<E Q L$

$<11 \quad U \quad<E Q L$

$<11 \quad U<E Q$

$<1 \quad U \quad<E Q$

$<11 \quad U \quad<E Q L$

$<11 \quad U<E Q L$

$<11 \quad U \quad<E Q$

$<E Q L$

$<E Q L$

$<E Q L$

$<E Q L$

$<E Q L$

$<$ EQL

$<E Q L$

$<E Q L$

$<E Q L$

$<$ EQL

$<$ EQL

$<E Q L$

$<E Q L$

$<$ EQL

$<$ EQL

$V<E Q L$

$<E Q L$

$<E Q L$

$V<E Q L$

$<E Q L$

$<E Q L$

$<E Q L$

$<E Q L$

$<E Q L$

$<E Q L$

$<E Q L$

$<$ EQL

$V<E Q L$

$<\mathrm{EQL}$

$<E Q L$

$<E Q L$

$<E Q L$

$<E Q L$

$<E Q L$

$<$ EQL.

$<E Q L$

$<E Q L$ 2nd Half CLP EPA Filt. Unit

ug/L

$\mathrm{ug} / \mathrm{L}$

$\mathrm{ug} / \mathrm{L}$

$\mathrm{ug} / \mathrm{L}$

$\mathrm{ug} / \mathrm{h}$

ug/L

$\mathrm{ug} / \mathrm{L}$

$\mathrm{ug} / \mathrm{L}$

ug/L

ug/L

$\mathrm{ug} / \mathrm{L}$

$\mathrm{ug} / \mathrm{L}$

$\mathrm{ug} / \mathrm{L}$

$\mathrm{ug} / \mathrm{L}$

ug/

$u g / L$

ug/L

ug/

ug/L

$\mathrm{ug} / \mathrm{L}$

$<.12$ JU $\quad<$ EQL ug/L

ug/L

$u g / L$

$\mathrm{ug} / \mathrm{L}$

$<5 \quad U<E Q$

$<5 \quad U<E Q$

$<5 \quad U<E Q$

$<5 \quad U<E Q$

$<10 \quad U \quad<E Q L$

$<5 \quad U \quad<E Q L$

$<5 \quad U<E Q$

$<5 \quad \mathrm{U} \quad<\mathrm{EQL}$

$<5 \quad U \quad<E Q L$

$<E Q L$

$<E Q L$

$<E Q L$

$<$ EQL

$<E Q L$

$<\mathrm{EQL}$

NDD

$<9.5 \quad \mathrm{U} \quad<\mathrm{EQL} \quad \mathrm{ug} / \mathrm{L}$

$u g / L$

$\mathrm{ug} / \mathrm{L}$

$\mathrm{ug} / \mathrm{L}$

$\mathrm{ug} / \mathrm{L}$

$\mathrm{ug} / \mathrm{L}$

ug/L

$u g / L$

$\mathrm{ug} / \mathrm{L}$

ug $/$

$\mathrm{ug} / \mathrm{L}$

$\mathrm{ug} / \mathrm{L}$

ug/

$\mathrm{ug} / \mathrm{L}$

ug/L

$u g / L$

ug/

ug/L

Notes: Concentrations in bold italics exceed the groundwater protection standards listed in Appendix A. Bold italics were not assigned if the result was qualified with a "J", "R", "L", or "U" qualifier.

$+=$ exceeded the Groundwater Protection Standards listed in Appendix A. 
Table B. Assessment Monitoring Results for Groundwater Wells (Cont.)

WELL LFW 43D

ANALYTICAL DATA

Groundwater Protection Standard

\section{ST Parameter}

Endrin aldehyde

Ethyl methacrylate

Ethyl methanesulfonate

Ethylbenzene

Famphur

Fluoranthene

Fluorene

Heptachlor

Heptachlor epoxide

Hexachlorobenzene

Hexachlorobutadiene

Hexachlorocyclopentadiene

Hexachloroethane

Hexachloropropene

Indeno(1,2,3-c,d)pyrene

lodomethane (Methyl iodide)

Isobutyl alcohol

Isodrin

Isophorone

Isosafrole

Kepone

Lead, total recoverable

Lindane

Mercury, total recoverable

Methacrylonitrile

Methapyrilene

Methoxychlor

Methyl ethyl ketone

Methyl isobutyl ketone

Methyl methacrylate

Methyl methanesulfonate

$\mathrm{N}$-Nitrosodi-n-butylamine

$\mathrm{N}$-Nitrosodiethylamine

$\mathrm{N}$-Nitrosodimethylamine

$\mathrm{N}$-Nitrosodiphenylamine

$\mathrm{N}-$ Nitrosodipropylamine

$\mathrm{N}$-Nitrosomethylethylamine

$\mathrm{N}$-Nitrosopiperidine

$\mathrm{N}$-Nitrosopyrrolidine

Naphthalene

Nickel, total recoverable

Nitrobenzene

O,O,O-Triethyl phosphorothioate

PCB

PCB 1016

PCB 1232

PCB 1242

PCB 1248

PCB 1254

PCB 1260

Parathion

Parathion methyl

Pentachlorobenzene

Pentachloronitrobenzene

Pentachlorophenol

Phenacetin

Phenanthrene

Phenol

Phorate

Pronamid

Propionitrile

Pyrene

Safrole

Selenium, total recoverable

Silver, total recoverable

Specific conductance

Styrene

Sulfide

\begin{tabular}{|c|c|c|}
\hline 1st Half & CLP EPA & Filt. \\
\hline$<.21$ & $u$ & $<\mathrm{EQL}$ \\
\hline$<5$ & $U$ & $<E C$ \\
\hline$<11$ & $u$ & $<\mathrm{E}$ \\
\hline$<5$ & $u$ & $<E C$ \\
\hline$<200$ & $U$ & $<\overline{E C}$ \\
\hline$<11$ & $U$ & $<E$ \\
\hline$<11$ & $u$ & $<E$ \\
\hline$<.1$ & $u$ & $<E C$ \\
\hline$<.1$ & $u$ & $<E$ \\
\hline$<11$ & $u$ & $<E$ \\
\hline$<11$ & $u$ & $<E C$ \\
\hline$<11$ & $u$ & $<E$ \\
\hline$<11$ & $u$ & $<\mathrm{EC}$ \\
\hline$<52$ & $u$ & $<E C$ \\
\hline$<11$ & $U$ & $<E C$ \\
\hline$<5$ & $u$ & $<\mathrm{EC}$ \\
\hline$<500$ & $U$ & $<\mathrm{EC}$ \\
\hline$<11$ & $u$ & $<\mathrm{EC}$ \\
\hline$<11$ & u & $<\mathrm{EC}$ \\
\hline$<11$ & $u$ & $<E C$ \\
\hline$<11$ & u & $<E C$ \\
\hline$<10$ & $u$ & $<\mathrm{EC}$ \\
\hline$<.1$ & $u$ & $<\mathrm{EC}$ \\
\hline$<.5$ & $u$ & $<\mathrm{EC}$ \\
\hline$<200$ & u & $<E C$ \\
\hline$<11$ & $u$ & $<\mathrm{EC}$ \\
\hline$<1$ & u & $<E C$ \\
\hline$<20$ & u & $<\mathrm{EC}$ \\
\hline$<10$ & U & $<\mathrm{EC}$ \\
\hline$<20$ & $u$ & $<E C$ \\
\hline$<11$ & U & $<E C$ \\
\hline$<11$ & $u$ & $<\mathrm{EC}$ \\
\hline$<11$ & $U$ & $<E C$ \\
\hline$<26$ & $u$ & $<\mathrm{EC}$ \\
\hline$<11$ & U & $<E$ \\
\hline$<11$ & $u$ & $<E O$ \\
\hline$<11$ & $u$ & $<E C$ \\
\hline$<11$ & $u$ & $<\mathrm{EC}$ \\
\hline$<11$ & $u$ & $<E C$ \\
\hline$<11$ & $U$ & $<\mathrm{E}$ \\
\hline$<50$ & U & $<E$ \\
\hline$<11$ & $u$ & $<\mathrm{E}$ \\
\hline$<11$ & $u$ & $<\mathrm{E}$ \\
\hline
\end{tabular}

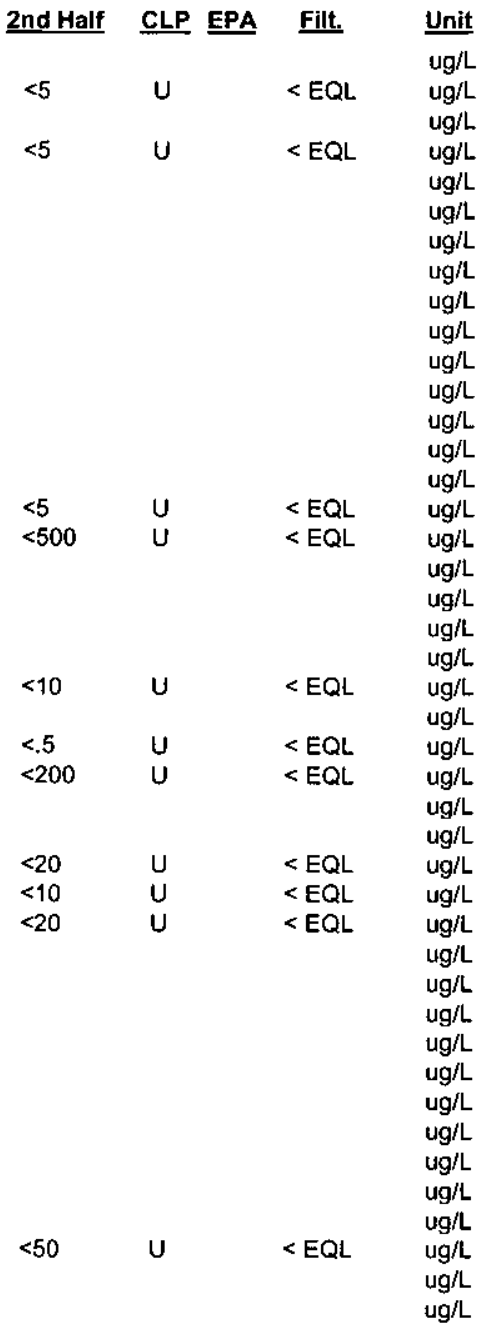

$\begin{array}{ccc}<11 & U & <E Q L \\ <11 & U & <E Q L \\ <52 & U & <E Q L \\ <11 & U & <E Q L \\ <26 & U & <E Q L \\ <11 & U & <\text { EQL } \\ <11 & U & <E Q L \\ <11 & U & <E Q L \\ <11 & U & <E Q L \\ <11 & U & <E Q L \\ <200 & U & <E Q L \\ <11 & U & <E Q L \\ <11 & U & <E Q L \\ <10 & U & <E Q L \\ <7.07 & J U & <E Q L \\ 9.8 & & <37 \\ <5 & U & <E Q L\end{array}$

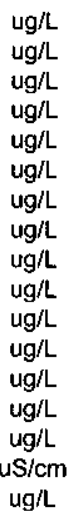

Notes: Concentrations in bold italics exceed the groundwater protection standards listed in Appendix A. Bold italics were not assigned if the result was qualified with a "J", "R", "L", or "U" qualifier.

$+=$ exceeded the Groundwater Protection Standards listed in Appendix A. 
Table B. Assessment Monitoring Results for Groundwater Wells (Cont.)

\section{WELL LFW 43D}

\section{ANALYTICAL DATA}

Groundwater Protection Standard

\section{ST Parameter}

Tetrachloroethylene

+ Thallium, total recoverable

Thionazin

Tin, total recoverable

Toluene

Toxaphene

Trichloroethylene

Trichlorofluoromethane

Vanadium, total recoverable

Vinyl acetate

Xy!enes

Zinc, total recoverable

alpha-Benzene hexachloride

alpha-Chlordane

beta-Benzene hexachloride

cis-1,2-Dichloroethylene

cis-1,3-Dichloropropene

delta-Benzene hexachloride

garnma-Chlordane

m-Cresol (3-Methylphenol)

$\mathrm{m}$-Nitroaniline

o-Cresol (2-Methylphenol)

o-Nitroaniline

o-Toluidine

$p, p=-D D D$

$p, p^{\prime \prime}-D D E$

p.p"-DDT

p-Cresol (4-Methylphenol)

p-Dimethylaminoazobenzene

p-Nitroaniline

p-Phenylenediamine

$\mathrm{pH}$

trans-1,2-Dichloroethylene

trans-1,3-Dichloropropene

trans-1,4-Dichloro-2-butene

\begin{tabular}{|c|c|c|c|}
\hline 1st Half & $\underline{\text { CLP }}$ & EPA & Filt. \\
\hline$<5$ & $U$ & & $<\mathrm{EQL}$ \\
\hline$<5.98$ & JU & & $<E Q L$ \\
\hline$<11$ & $U$ & & $<E Q L$ \\
\hline$<200$ & U & & $<E Q L$ \\
\hline$<5$ & $U$ & & $<E Q L$ \\
\hline$<2.1$ & $\mathrm{U}$ & & $<\mathrm{EQL}$ \\
\hline$<5$ & U & & $<E Q L$ \\
\hline$<5$ & $\mathrm{U}$ & & $<E Q L$ \\
\hline$<10$ & $U$ & & $<\mathrm{EQL}$ \\
\hline$<5$ & U & & $<\mathrm{EQL}$ \\
\hline$<10$ & U & & $<$ EQL \\
\hline 13.5 & $J$ & I & NDD \\
\hline$<.1$ & $\mathbf{U}$ & & $<\mathrm{EQL}$ \\
\hline$<.1$ & U & & $<\mathrm{EQL}$ \\
\hline$<.1$ & $\mathrm{U}$ & & $<\mathrm{EQL}$ \\
\hline$<5$ & $\mathrm{U}$ & & $<\mathrm{EQL}$ \\
\hline$<5$ & $U$ & & $<$ EQL \\
\hline$<.1$ & $U$ & & $<$ EQL \\
\hline$<.1$ & $U$ & & $<$ EQL \\
\hline$<26$ & $U$ & & $<\mathrm{EQL}$ \\
\hline$<11$ & $U$ & & $<$ EQL \\
\hline$<26$ & $\mathrm{U}$ & & $<\mathrm{EQL}$ \\
\hline$<11$ & $U$ & & $<E Q L$ \\
\hline$<.21$ & U & & $<E Q L$ \\
\hline$<.21$ & U & & $<\mathrm{EQL}$ \\
\hline$<.21$ & $U$ & & $<\mathrm{EQL}$ \\
\hline$<10$ & $U$ & & $<E Q L$ \\
\hline$<11$ & U & & $<\mathrm{EQL}$ \\
\hline$<11$ & U & & $<$ EQL \\
\hline$<11$ & U & & $<\mathrm{EQL}$ \\
\hline 5.75 & $\mathrm{~J}$ & $Q$ & NDD \\
\hline$<5$ & U & & $<$ EQL \\
\hline$<5$ & U & & $<\mathrm{EQL}$ \\
\hline$<20$ & $U$ & & $<\mathrm{EQL}$ \\
\hline
\end{tabular}

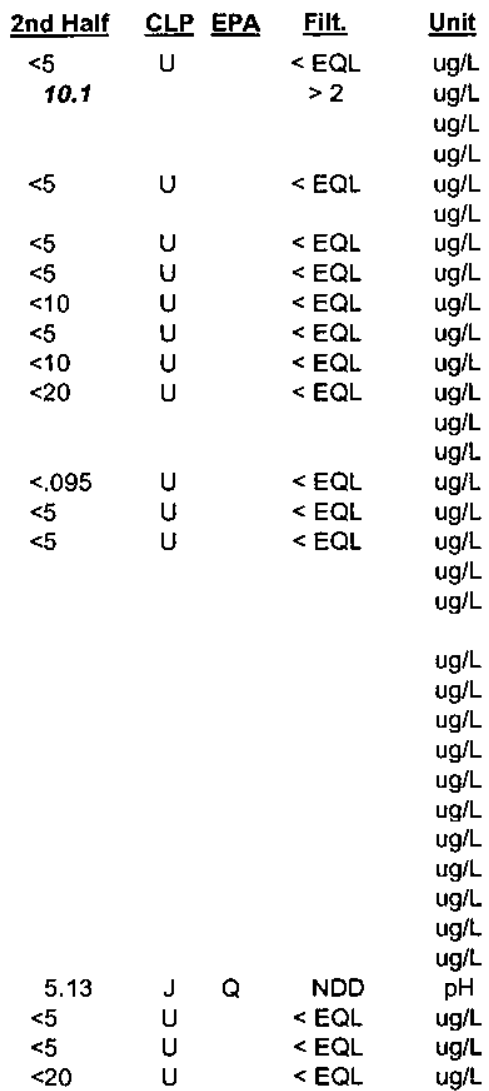

Notes: Concentrations in bold Italics exceed the groundwater protection standards listed in. Appendix A. Bold italics were not assigned if the result was qualified with a "J", "R", "L", or "U" qualifier.

$+=$ exceeded the Groundwater Protection Standards listed in Appendix A. 
Table B. Assessment Monitoring Results for Groundwater Wells (Cont.)

WELL LFW 74C

$\begin{array}{ll}\text { SRS Coord. } & \text { Lat/Lonqitude } \\ \text { N 85813.8 } & \text { 33.28914 Deg N } \\ \text { E 45097.8 } & -81.7147 \text { Deg W }\end{array}$

SAMPLE DATE

FIELD DATA

Parameter

Water Elevation

$\mathrm{pH}$

Sp. Conductance

Water temperature

Alkalinity as $\mathrm{CaCO} 3$

Turbidity

Volumes purged

Sampling code

\section{ANALYTICAL DATA}

Groundwater Protection Standard

ST Parameter

1,1,1,2-Tetrachloroethane

1,1,1-Trichloroethane

1,1,2,2-Tetrachloroethane

1,1,2-Trichloroethane

1,1-Dichloroethane

1,1-Dichloroethylene

1,1-Dichloropropene

1,2,3-Trichloropropane

1,2,4,5-Tetrachlorobenzene

1,2,4-Trichlorobenzene

1,2-Dibromo-3-chloropropane

1,2-Dibromoethane

1,2-Dichlorobenzene

1,2-Dichloroethane

1,2-Dichloropropane

1,3,5-Trinitrobenzene

1,3-Dichlorobenzene

1,3-Dichloropropane

1,3-Dinitrobenzene

1,4-Dichlorobenzene

1,4-Naphthoquinone

1-Naphthylamine

2,2-Dichloropropane

2,2-Oxybis(1-chloropropane)

2,3,4,6-Tetrachlorophenol

2,4,5-T

2,4,5-TP (Silvex)

2,4,5-Trichlorophenol

2,4,6-Trichlorophenol

2,4-Dichlorophenol

2,4-Dichlorophenoxyacetic acid

2,4-Dimethyl phenol

2,4-Dinitrophenol

2,4-Dinitrotoluene

2,6-Dichlorophenol

2,6-Dinitrotoluene

2-Acetylaminofluorene

2-Chloronaphthalene

2-Chlorophenol

2-Hexanone

2-Methyl-4,6-dinitrophenol

2-Methylnaphthalene

2-Naphthylamine

2-Nitrophenol

2-sec-Butyl-4,6-dinitrophenol

$3,3^{\mathrm{H}}$-Dichlorobenzidine

3,3"-Dimethylbenzidine

3-Methylcholanthrene

4-Aminobiphenyl

4-Bromophenyl phenyl ether

4-Chloro-m-cresol

\author{
Screen Zone Elevation \\ 116-101 ft msl
}

03/08/00

1st Half
160.8
5
35
19.2
4
.8
3.89

\begin{tabular}{l} 
1st Half \\
\hline$<5$ \\
$<5$ \\
$<5$ \\
$<5$ \\
$<5$ \\
$<5$ \\
$<5$ \\
$<5$ \\
$<5$ \\
$<53$ \\
$<11$ \\
$<10$ \\
$<5$ \\
$<5$ \\
$<5$ \\
$<5$ \\
$<10$ \\
$<5$ \\
$<5$ \\
$<50$ \\
$<5$ \\
$<11$ \\
$<11$ \\
$<5$ \\
$<11$ \\
$<11$ \\
$<.2$ \\
$<.2$ \\
$<11$ \\
$<26$ \\
$<11$ \\
$<.2$ \\
$<11$ \\
$<26$ \\
$<11$ \\
$<25$ \\
$<11$ \\
$<11$ \\
$<11$ \\
$<11$ \\
$<11$ \\
$<20$ \\
$<26$ \\
$<11$ \\
$<11$ \\
$<11$ \\
$<.2$ \\
$<11$ \\
$<21$ \\
$<11$ \\
$<11$ \\
$<11$ \\
$<11$ \\
\end{tabular}

$\frac{\text { Top of Casing }}{213.6 \mathrm{ft} \mathrm{msl}}$

Casing

4 " PVC

Pump

Screen Zone

$10 / 02 / 00$

$\begin{array}{ll}\text { 2nd Half } & \text { Unit } \\ 202.18 & \text { ft msl } \\ 5.1 & \mathrm{pH} \\ 35 & \text { uS/cm } \\ 21 & \text { deg. C } \\ 0 & \text { mg/L } \\ .5 & \text { NTU } \\ 2.57 & \text { well volume }\end{array}$

Middle

\begin{tabular}{cl} 
CLP EPA & Filt. \\
\hline$U$ & $<E Q L$ \\
$U$ & $<E Q L$ \\
$U$ & $<E Q L$ \\
$U$ & $<E Q L$ \\
$U$ & $<E Q L$ \\
$U$ & $<E Q L$ \\
$U$ & $<E Q L$ \\
$U$ & $<E Q L$
\end{tabular}

$\underline{\text { Unit }}$

$Q<E Q L$

$<E Q L$

$<E Q L$

$<E Q L$

$<E Q L$

$<E Q L$

$<E Q L$

$Q<E Q L$

$<$ EQL

$<$ EQL

$Q<E Q L$

$<E Q L$

$<\mathrm{EQL}$

$<E Q L$

$<$ EQL

$<E Q L$

$<E Q L$

$<$ EQL

$<$ EQL

$<E Q L$

$<E Q L$

$<E Q L$

$<$ EQL

$<E Q L$

$<$ EQL

$<E Q L$

$<E Q L$

$<E Q L$

$<E Q L$

$<$ EQL

$<E Q L$

$<E Q L$

$<\mathrm{EQL}$

$<$ EQL

$<E Q L$

$<$ EQL

$<E Q L$

$<E Q L$

$<E Q L$

$<\mathrm{EQL}$

$<$ EQL

$<E Q L$

$<E Q L$

2nd Half
$<5$
$<5$
$<5$
$<5$
$<5$
$<5$
$<5$
$<5$

CLP EPA

$\quad$ Filt.
$<E Q L$
$<E Q L$
$<E Q L$
$<E Q L$
$<E Q L$
$<E Q L$
$<E Q L$
$<E Q L$

ug/L

ug/h

$\mathrm{ug} / \mathrm{L}$

ug/L

$u g / L$

$u g / L$

$u g / L$

$u g / L$

ugh

$u g / L$

$\begin{array}{llll}<10 & \mathrm{U} & <\mathrm{EQL} & \mathrm{ug} / \mathrm{L} \\ <5 & \mathrm{U} & <\mathrm{EQL} & \mathrm{Ug} / \mathrm{L}\end{array}$

$<5<U<1$

$\begin{array}{llll}<5 & U & <E Q L & \mathrm{Ug} / \mathrm{L} \\ <5 & \mathrm{E} & <\mathrm{EQL} & \mathrm{ug} / \mathrm{L}\end{array}$

ug/L

$\begin{array}{llll}<5 & U & <E Q L & u g / L \\ <5 & U & <E Q L & u g / L\end{array}$

ug/L

$<5 \quad U<E Q L \quad \begin{aligned} & u g / L \\ & \mathrm{ug} / \mathrm{L}\end{aligned}$

$<5 \quad U \quad<g / L$

$4<\mathrm{EQL} \quad \mathrm{ug} / \mathrm{h}$

$\mathrm{ug} / \mathrm{L}$

$\mathrm{ug} / \mathrm{L}$

$\mathrm{ug} / \mathrm{L}$

$\mathrm{ug} / \mathrm{L}$

ug/L

$\mathrm{ug} / \mathrm{L}$

$\mathrm{ug} / \mathrm{L}$

$\mathrm{ug} / \mathrm{L}$

$\mathrm{ug} / \mathrm{L}$

ug/L

ug/L

ugh

ug $/ L$

ug/L

$\mathrm{ug} / \mathrm{L}$

$\mathrm{ug} / \mathrm{L}$

$\mathrm{ug} / \mathrm{L}$

$\mathrm{ug} / \mathrm{L}$

$\mathrm{ug} / \mathrm{L}$

$\mathrm{ug} / \mathrm{L}$

ug/L

ug/L

ugh $/ \mathrm{L}$

$\mathrm{ug} / \mathrm{L}$

ug/L

ug/L

ug/L

Notes: Concentrations in bold italics exceed the groundwater protection standards listed in Appendix A. Bold italics were not assigned if the result was qualified with a "J", "R", "L", or "U" qualifier.

$+=$ exceeded the Groundwater Protection Standards listed in Appendix A. 
Table B. Assessment Monitoring Results for Groundwater Wells (Cont.)

WELL LFW 74C

ANALYTICAL DATA

Groundwater Protection Standard

ST Parameter

4-Chloroaniline

4-Chlorophenyl phenyl ether

4-Nitrophenol

5-Nitro-o-toluidine

7,12-Dimethylbenz(a)anthracene

Acenaphthene

Acenaphthylene

Acetone

Acetonitrile (Methyl cyanide)

Acetophenone

Acrolein

Acrylonitrile

Aldrin

Allyl chloride

Anthracene

Antimony, total recoverable

Arsenic, total recoverable

Barium, total recoverable

Benzene

Benzo(a)anthracene

Benzo(a)pyrene

Benzo(b)fluoranthene

Benzo(g,h,i)perylene

Benzo(k)fluoranthene

Benzyl alcohol

Beryllium, total recoverable

Bis (2-chloroethoxy) methane

Bis(2-chloroethyl) ether

Bis(2-ethylhexyl) phthalate

Bromochloromethane

Bromodichloromethane

Bromoform

Bromomethane (Methyl bromide)

Butylbenzyl phthalate

Cadmium, total recoverable

Carbon disulfide

Carbon tetrachloride

Chlorobenzene

Chlorobenzilate

Chloroethane

Chloroethene (Vinyl chloride)

Chloroform

Chloromethane (Methyl chloride)

Chloroprene

Chromium, total recoverable

Chrysene

Cobalt, total recoverable

Copper, total recoverable

Cyanide

Di-n-butyl phthalate

Di-n-octyl phthalate

Diallate

Dibenz(a,h)anthracene

Dibenzofuran

Dibromochloromethane

Dibromomethane (Methylene bromide)

Dichlorodiffuoromethane

Dichloromethane (Methylene chloride)

Dieldrin

Diethyl phthalate

Dimethoate

Dimethyl phthalate

Diphenylamine

Disulfoton

Endosulfan I

Endosulfan II

Endosulfan sulfate

Endrin

1st

$<11$

$<11$

$<11$ JU $Q \quad<E Q L$

$<11$ JU $Q<E Q L$

$<11$ JU $Q \quad<E Q L$

$<11$ JU $Q<E Q L$

$<20 \quad U<E Q L$

$<200 \quad U<E Q L$

$<11$ JU $Q<E Q L$

$<50 \quad U \quad<$ EQL

$<10 \quad U \quad<$ EQL

$<.1 \quad U<E Q L$

$<5 \quad U<E Q L$

$<11$ JU $Q<E Q L$

$<100 \quad U<E Q$

$<10 \quad U \quad<$ EQL

5.97 J I NDD

$<5 \quad U \quad<E Q L$

$<11$ JU $Q \quad<E Q L$

$<11$ JU Q $<E Q L$

$<11$ JU $Q<E Q L$

$<11$ JU $Q<E Q L$

$<11$ JU $Q<E Q L$

$<1 \quad U<E Q$

$<11$ JU $Q \quad<E Q$

$<11$ JU $Q<E Q L$

$<11$ JU $Q<E Q L$

$<5 \quad U \quad<E Q L$

$<5 \quad U<E Q L$

$<5 \quad U \quad<E Q L$

$<5 \quad U \quad<E Q L$

$<11$ JU $Q \quad<E Q L$

$<10 \quad U<E Q$

$<5 \quad U \quad<$ EQL

$<5 \quad U<$ \&QL

$<5 \quad U<E Q$

$<11$ JU $Q \quad<E Q L$

$<5 \quad U<$ EQL

$<5 \quad U<E Q L$

$<5 \quad U \quad<E Q L$

$<5 \quad U$

$<20 \quad U<<E Q$

$<10 \quad U<E Q$

$<11$ JU $Q \quad<E Q L$

$<20 \quad U \quad<E Q L$

$<20 \quad U<$ EQL

10 J Q NDD

$<11$ JU $Q<E Q L$

$<11$ JU $Q<E Q L$

$<11$ JU $Q<E Q L$

$<11$ JU $Q \quad<E Q L$

$<11$ JU $Q<E Q$

$<5 \quad U<E Q L$

$<5 \quad U<<$ <QL

$<5 \quad U<\mathrm{EQL}$

$1.5 \mathrm{~J} \quad \mathrm{NDD}$

$<.21$

$<11$

$<11$

$<11$

$<11$

$<11$

$<.1$

$<.21$

$<.21$

$<.21$

\begin{tabular}{|c|c|c|c|}
\hline 2nd Haif & CLP & EPA & Filt. \\
\hline$<20$ & $U$ & & $<E Q L$ \\
\hline$<200$ & U & & $<\mathrm{EQL}$ \\
\hline$<50$ & $U$ & & $<$ EQL \\
\hline$<10$ & U & & $<E Q L$ \\
\hline$<5$ & $U$ & & $<E Q L$ \\
\hline$<100$ & U & & $<E Q L$ \\
\hline$<10$ & U & & $<E Q L$ \\
\hline 5.65 & $\mathrm{~J}$ & 1 & NDD \\
\hline$<5$ & U & & $<E Q L$ \\
\hline
\end{tabular}

Unit

ug/L

ug/L

$u g / L$

$u g / L$

ug $/ \mathrm{L}$

ug/L

ug/L

ug/L

$u g / L$

$\mathrm{ug} / \mathrm{L}$

$\mathrm{ug} / \mathrm{L}$

$\mathrm{ug} / \mathrm{L}$

ug/L

ug/L

$\mathrm{ug} / \mathrm{L}$

ug/L

ug $/ \mathrm{L}$

$\mathrm{ug} / \mathrm{L}$

$u g / L$

$u g / L$

$\mathrm{ug} / \mathrm{h}$

$u g /$ L

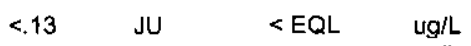

ug/L

$\mathrm{ug} / \mathrm{L}$

ug/L

ug/L

$\mathrm{ug} / \mathrm{L}$

$\mathrm{ug} / \mathrm{L}$

$u g / L$

ug/h

$\mathrm{ug} / \mathrm{L}$

ug/L

ug/L

$\mathrm{ug} / \mathrm{h}$

$\mathrm{ug} / \mathrm{L}$

$\mathrm{ug} / \mathrm{L}$

ug/L

$\mathrm{ug} / \mathrm{L}$

ug/L

$\mathrm{ug} / \mathrm{L}$

$\mathrm{ug} / \mathrm{L}$

ugh

$\mathrm{ug} / \mathrm{L}$

$u g h$

$u g / L$

$\mathrm{ug} / \mathrm{L}$

ug/L

$\mathrm{ug} / \mathrm{L}$

ug/L

ug/L

$\mathrm{ug} / \mathrm{L}$

ug/

ug/L

ug/

ug $/ \mathrm{L}$

$\mathrm{ug} / \mathrm{L}$

ug/ $/$

ug/h

ug/t

$u g / L$

ug/

ug/L

$\mathrm{ug} / \mathrm{L}$

Notes: Concentrations In bold italics exceed the groundwater protection standards listed in Appendix A. Bold ltalics were not assigned if the result was qualified with a "J", "R", "L", or "U" qualifier.

$+=$ exceeded the Groundwater Protection Standards listed in Appendix A. 
Table B. Assessment Monitoring Results for Groundwater Wells (Cont.)

WELL LFW 74C

ANALYTICAL DATA

Groundwater Protection Standard

\section{ST Parameter}

Endrin aldehyde

Ethyl methacrylate

Ethyl methanesulfonate

Ethylbenzene

Famphur

Fluoranthene

Fluorene

Heptachlor

Heptachlor epoxide •

Hexachlorobenzene

Hexachlorobutadiene

Hexachlorocyclopentadiene

Hexachloroethane

Hexachloropropene

Indeno(1,2,3-c,d)pyrene

lodomethane (Methyl iodide)

Isobutyl alcoho

Isodrin

Isophorone

Isosafrole

Kepone

Lead, total recoverable

Lindane

Mercury, total recoverable

Methacrylonitrile

Methapyrilene

Methoxychlor

Methyl ethyl ketone

Methyl isobutyl ketone

Methyl methacrylate

Methyl methanesulfonate

N-Nitrosodi-n-butylamine

$\mathrm{N}$-Nitrosodiethylamine

$\mathrm{N}$-Nitrosodimethylamine

$\mathrm{N}$-Nitrosodiphenylamine

N-Nitrosodipropylamine

$\mathrm{N}$-Nitrosomethylethylamine

$\mathrm{N}$-Nitrosopiperidine

$\mathrm{N}$-Nitrosopyrrolidine

Naphthalene

Nickel, total recoverable

Nitrobenzene

O,O,O-Triethyl phosphorothioate

PCB

PCB 1016

PCB 1232

PCB 1242

PCB 1248

PCB 1254

PCB 1260

Parathion

Parathion methyl

Pentachlorobenzene

Pentachloronitrobenzene

Pentachlorophenol

Phenacetin

Phenanthrene

Phenol

Phorate

Pronamid

Propionitrile

Pyrene

Safrole

Selenium, total recoverable

Silver, total recoverable

Specific conductance

Styrene

Sulfide

\begin{tabular}{|c|c|c|c|}
\hline 1st Half & CLP & EPA & Filt. \\
\hline$<.21$ & $U$ & & $<E Q L$ \\
\hline$<5$ & $U$ & & $<\mathrm{EQL}$ \\
\hline$<11$ & JU & $Q$ & $<E Q L$ \\
\hline$<5$ & U & & $<\mathrm{EQL}$ \\
\hline$<200$ & JU & $Q$ & $<E Q L$ \\
\hline$<11$ & JU & $Q$ & $<E Q L$ \\
\hline$<11$ & JU & $Q$ & $<E Q L$ \\
\hline$<.1$ & U & & $<E Q L$ \\
\hline$<.1$ & U & & $<\mathrm{EQL}$ \\
\hline$<11$ & JU & $Q$ & $<\mathrm{EQL}$ \\
\hline$<11$ & JU & $Q$ & $<E Q L$ \\
\hline$<11$ & JU & $Q$ & $<E Q L$ \\
\hline$<11$ & JU & $Q$ & $<\mathrm{EQL}$ \\
\hline$<53$ & JU & $\mathbf{Q}$ & $<\mathrm{EQL}$ \\
\hline$<11$ & JU & $\mathbf{Q}$ & $<\mathrm{EQL}$ \\
\hline$<5$ & u & & $<E Q L$ \\
\hline$<500$ & $U$ & & $<\mathrm{EQL}$ \\
\hline$<11$ & JU & $Q$ & $<\mathrm{EQL}$ \\
\hline$<11$ & JU & $Q$ & $<E Q L$ \\
\hline$<11$ & JU & $Q$ & $<\mathrm{EQL}$ \\
\hline$<11$ & JU & $Q$ & $<$ EQL \\
\hline 4.21 & J & 1 & NDD \\
\hline$<.1$ & $\mathrm{U}$ & & $<\mathrm{EQL}$ \\
\hline$<.154$ & u & V & $<\mathrm{EQL}$ \\
\hline$<200$ & $U$ & & $<\mathrm{EQL}$ \\
\hline$<11$ & JU & $Q$ & $<\mathrm{EQL}$ \\
\hline$<1$ & U & & $<E Q L$ \\
\hline$<20$ & $U$ & & $<E Q L$ \\
\hline$<10$ & u & & $<\mathrm{EQL}$ \\
\hline$<20$ & U & & $<$ EQL \\
\hline$<11$ & JU & $Q$ & $<E Q L$ \\
\hline$<11$ & JU & $Q$ & $<E Q L$ \\
\hline$<11$ & JU & $Q$ & $<E Q L$ \\
\hline$<26$ & JU & $Q$ & $<\mathrm{EQL}$ \\
\hline$<11$ & JU & $Q$ & $<E Q L$ \\
\hline$<11$ & JU & $Q$ & $<E Q L$ \\
\hline$<11$ & JU & $Q$ & $<\mathrm{EQL}$ \\
\hline$<11$ & JU & $Q$ & $<E Q L$ \\
\hline$<11$ & JU & $Q$ & $<E Q L$ \\
\hline$<11$ & JU & $Q$ & $<E Q L$ \\
\hline$<50$ & $u$ & & $<E Q L$ \\
\hline$<11$ & JU & $\mathbf{Q}$ & $<\mathrm{EQL}$ \\
\hline$<11$ & JU & $Q$ & $<E Q L$ \\
\hline$<1$ & U & & $<\mathrm{EQL}$ \\
\hline$<1$ & U & & $<\mathrm{EQL}$ \\
\hline$<2,1$ & $u$ & & $<$ EQL \\
\hline$<1$ & $U$ & & $<E Q L$ \\
\hline$<1$ & $U$ & & $<E Q L$ \\
\hline$<1$ & U & & $<\mathrm{EQL}$ \\
\hline$<11$ & JU & $Q$ & $<E Q L$ \\
\hline$<11$ & JU & $Q$ & $<E Q L$ \\
\hline$<53$ & JU & $Q$ & $<E Q L$ \\
\hline$<11$ & JU & $Q$ & $<E Q L$ \\
\hline$<26$ & JU & $Q$ & $<\mathrm{EQL}$ \\
\hline$<11$ & JU & $\mathbf{Q}$ & $<E Q L$ \\
\hline$<11$ & JU & $Q$ & $<E Q L$ \\
\hline$<11$ & JU & $Q$ & $<E Q L$ \\
\hline$<11$ & JU & $Q$ & $<\mathrm{EQL}$ \\
\hline$<11$ & JU & $Q$ & $<E Q L$ \\
\hline$<200$ & U & & $<E Q L$ \\
\hline$<11$ & JU & $Q$ & $<E Q L$ \\
\hline$<11$ & JU & $Q$ & $<\mathrm{EQL}$ \\
\hline$<10$ & $U$ & & $<E Q L$ \\
\hline$<20$ & U & & $<E Q L$ \\
\hline 33.4 & & & $<37$ \\
\hline$<5$ & $U$ & & $<E Q L$ \\
\hline$<1000$ & JU & $Q$ & $<\mathrm{EQL}$ \\
\hline
\end{tabular}

Notes: Concentrations in bold italics exceed the groundwater protection standards listed in Appendix $A$. Bold italics were not assigned if the result was qualified with a "J", "R", "L", or "U" quallfier.

$+=$ exceeded the Groundwater Protection Standards listed in Appendix A. 
Table B. Assessment Monitoring Results for Groundwater Wells (Cont.)

WELL LFW 74C

ANALYTICAL DATA

Groundwater Protection Standard

\section{ST Parameter}

Tetrachloroethylene

Thallium, total recoverable

Thionazin

Tin, total recoverable

Toluene

Toxaphene

Trichloroethylene

Trichlorofluoromethane

Vanadium, total recoverable

Vinyl acetate

Xylenes

Zinc, total recoverable

alpha-Benzene hexachloride

alpha-Chlordane

beta-Benzene hexachloride

cis-1,2-Dichloroethylene

cis-1,3-Dichloropropene

delta-Benzene hexachloride

gamma-Chlordane

m-Cresol (3-Methylphenol)

$\mathrm{m}$-Nitroaniline

o-Cresol (2-Methylphenol)

o-Nitroaniline

o-Toluidine

p,p"-DDD

$p, p^{\prime \prime-D D E ~}$

p.p"-DDT

p-Cresol (4-Methylphenol)

p-Dimethylaminoazobenzene

p-Nitroaniline

p-Phenylenediamine

$\mathrm{pH}$

trans-1,2-Dichloroethylene

trans-1,3-Dichloropropene

trans-1,4-Dichloro-2-butene

\begin{tabular}{|c|c|c|c|}
\hline 1st Hal & C노 & EPA & Filt. \\
\hline$<5$ & U & & $<E Q L$ \\
\hline$<10$ & U & & $<E Q L$ \\
\hline$<11$ & JU & $Q$ & $<\mathrm{EQL}$ \\
\hline$<200$ & U & & $<\mathrm{EQL}$ \\
\hline$<5$ & $U$ & & $<E Q L$ \\
\hline$<2.1$ & U & & $<\overline{E Q L}$ \\
\hline$<5$ & U & & $<E Q L$ \\
\hline$<5$ & $U$ & & $<E Q L$ \\
\hline$<10$ & $U$ & & $<E Q L$ \\
\hline$<5$ & U & & $<E Q L$ \\
\hline$<10$ & $U$ & & $<E Q L$ \\
\hline 14.8 & $J$ & I & NDD \\
\hline$<.1$ & $U$ & & $<E Q L$ \\
\hline$<.1$ & U & & $<$ EQL \\
\hline$<.1$ & U & & $<E Q L$ \\
\hline$<5$ & U & & $<E Q L$ \\
\hline$<5$ & U & & $<\mathrm{EQL}$ \\
\hline$<.1$ & U & & $<\mathrm{EQ}$ \\
\hline$<.1$ & U & & $<\mathrm{EQL}$ \\
\hline$<26$ & JU & $Q$ & $<E Q L$ \\
\hline$<11$ & JU & $Q$ & $<\mathrm{EQL}$ \\
\hline$<26$ & JU & $\vec{Q}$ & $<\mathrm{EQL}$ \\
\hline$<11$ & JU & $Q$ & $<$ EQL \\
\hline$<.21$ & U & & $<\mathrm{EQ}$ \\
\hline$<.21$ & U & & $<E Q L$ \\
\hline$<.21$ & $U$ & & $<E Q L$ \\
\hline$<10$ & JU & $Q$ & $<$ EQL \\
\hline$<11$ & JU & $Q$ & $<E Q L$ \\
\hline$<11$ & JU & $Q$ & $<E Q L$ \\
\hline$<11$ & JU & $Q$ & $<\mathrm{EQ}$ \\
\hline 5.7 & $\mathrm{~J}$ & $\mathbf{Q}$ & ND \\
\hline$<5$ & U & & < EQI \\
\hline$<5$ & U & & $<\mathrm{EQ}$ \\
\hline$<20$ & U & & $<\mathrm{EQ}$ \\
\hline
\end{tabular}

\begin{tabular}{|c|c|c|c|c|}
\hline 2nd Half & CLP & EPA & Filt. & Unit \\
\hline$<5$ & $u$ & & $<\mathrm{EQL}$ & ug/L \\
\hline$<10$ & $u$ & & $<\mathrm{EQL}$ & ug/L. \\
\hline & & & & $\mathrm{ug} / \mathrm{L}$ \\
\hline$<5$ & U & & $<$ EQL & $\mathrm{ug} / \mathrm{L}$ \\
\hline & & & & $\mathrm{Ug} / \mathrm{L}$ \\
\hline$<5$ & U & & $<E Q L$ & ug/L. \\
\hline$<5$ & U & & $<\mathrm{EQL}$ & $\mathrm{ug} / \mathrm{L}$ \\
\hline$<10$ & U & & $<E Q L$ & ug/L \\
\hline$<5$ & U & & $<$ EQL & $\mathrm{ug} / \mathrm{L}$ \\
\hline$<10$ & U & & $<E Q L$ & $u g / L$ \\
\hline$<20$ & U & & $<E Q L$ & $\mathrm{ug} / \mathrm{L}$ \\
\hline & & & & ug/L \\
\hline$<.1$ & $U$ & & $<E Q L$ & $u g / L$ \\
\hline$<5$ & U & & $<E Q L$ & ug/L \\
\hline \multirow[t]{11}{*}{$<5$} & $\Psi$ & & $<E Q L$ & $\mathrm{ug} / \mathrm{L}$ \\
\hline & & & & $\mathrm{ug} / \mathrm{L}$ \\
\hline & & & & $\mathrm{ug} / \mathrm{L}$ \\
\hline & & & & $u g / L$ \\
\hline & & & & $\begin{array}{l}\mathrm{ug} / \mathrm{L} \\
\mathrm{ug} / \mathrm{L}\end{array}$ \\
\hline & & & & $\mathrm{ug} / \mathrm{L}$ \\
\hline & & & & ug/L \\
\hline & & & & ug/L. \\
\hline & & & & $\mathrm{ug} / \mathrm{L}$ \\
\hline & & & & $\mathrm{ug} / \mathrm{L}$ \\
\hline & & & & 1 \\
\hline 4.97 & $J$ & a & NDD & $\mathrm{pH}$ \\
\hline$<5$ & $U$ & & $<\mathrm{EQL}$ & $\mathrm{ug} / \mathrm{L}$ \\
\hline$<5$ & U & & $<E Q L$ & $\mathrm{ug} / \mathrm{L}$ \\
\hline$<20$ & U & & $<E Q L$ & $\mathrm{ug} / \mathrm{L}$ \\
\hline
\end{tabular}

\footnotetext{
Notes: Concentrations in bold italics exceed the groundwater protection standards tisted in Appendix A. Bold italics were not assigned if the result was qualified with a "J", "R", "L", or "U" qualifier.

$+=$ exceeded the Groundwater Protection Standards listed in Appendix A.
} 
Table B. Assessment Monitoring Results for Groundwater Wells (Cont.)

WELL LFW 74D

\begin{tabular}{|c|c|c|c|c|c|c|}
\hline SRS Coord. & Lat/Longitude & Screen Zone Elevation & Top of Casing & Casing & Pump & Screen Zone \\
\hline $\begin{array}{l}\text { N } 85828.1 \\
\text { E } 45098\end{array}$ & $\begin{array}{l}33.28917 \mathrm{Deg} N \\
-81.7148 \mathrm{Deg} W\end{array}$ & $167.7-152.7 \mathrm{ft} \mathrm{msl}$ & $213.9 \mathrm{ft} \mathrm{msl}$ & $4 " \mathrm{PVC}$ & $s$ & Upper \\
\hline SAMPLE DATE & & $03 / 08 / 00$ & & $10 / 03 / 00$ & & \\
\hline \multicolumn{7}{|l|}{ FIELD DATA } \\
\hline Parameter & & 1st Half & & 2nd Half & Unit & \\
\hline $\begin{array}{l}\text { Water Elevation } \\
\text { pH } \\
\text { Sp. Conductance } \\
\text { Water temperature } \\
\text { Alkalinity as } \mathrm{CaCO} 3 \\
\text { Turbidity } \\
\text { Volumes purged }\end{array}$ & & $\begin{array}{l}160.9 \\
5.2 \\
25 \\
19.9 \\
4 \\
3.2 \\
5.72\end{array}$ & & $\begin{array}{l}160.3 \\
4.9 \\
19 \\
20.3 \\
0 \\
1.8 \\
10.3\end{array}$ & $\begin{array}{l}\text { ft msl } \\
\mathrm{pH} \\
\text { uS/cm } \\
\text { deg. C } \\
\text { mg/L } \\
\text { NTU } \\
\text { well vol }\end{array}$ & \\
\hline
\end{tabular}

\section{ANALYTICAL DATA}

Groundwater Protection Standard

\section{SI Parameter}

1,1,1,2-Tetrachloroethane

1,1,1-Trichloroethane

1,1,2,2-Tetrachloroethane

1,1,2-Trichloroethane

1,1-Dichloroethane

1,1-Dichloroethylene

1,1-Dichloropropene

1,2,3-Trichloropropane

$1,2,4,5$-Tetrachiorobenzene

1,2,4-Trichlorobenzene

1,2-Dibromo-3-chloropropane

1,2-Dibromoethane

1,2-Dichlorobenzene

1,2-Dichloroethane

1,2-Dichtoropropane

1,3,5-Trinitrobenzene

1,3-Dichlorobenzene

1,3-Dichloropropane

1,3-Dinitrobenzene

1,4-Dichlorobenzene

1,4 -Naphthoquinone

1-Naphthylamine

2,2-Dichloropropane

2,2-Oxybis(1-chloropropane)

2,3,4,6-Tetrachlorophenol

2,4,5-T

2,4,5-TP (Silvex)

2,4,5-Trichlorophenol

2,4,6-Trichlorophenol

2,4-Dichlorophenol

2,4-Dichlorophenoxyacetic acid

2,4-Dimethyl phenol

2,4-Dinitrophenol

2,4-Dinitrotoluene

2,6-Dichlorophenol

2,6-Dinitrotoluene

2-Acetylaminofluorene

2-Chloronaphthalene

2-Chlorophenol

2-Hexanone

2-Methyl-4,6-dinitrophenol

2-Methylnaphthalene

2-Naphthylamine

2-Nitrophenol

2-sec-Butyl-4,6-dinitrophenol

3,3"--Dichlorobenzidine

3,3"-Dimethylbenzidine

3-Methylcholanthrene

4-Aminobipheny

4-Bromophenyl phenyl ether

4-Chloro-m-cresol

\begin{tabular}{|c|c|c|c|}
\hline 1st Half & CLP & EP & Fitt \\
\hline$<5$ & U & & $<E Q$ \\
\hline 3.8 & $\mathrm{~J}$ & I & ND \\
\hline$<5$ & U & & $<\mathrm{EQ}$ \\
\hline$<5$ & U & & $<E Q$ \\
\hline 3 & $\mathrm{~J}$ & 1 & ND \\
\hline$<5$ & U & & $<\mathrm{EQ}$ \\
\hline$<5$ & U & & $<\mathrm{EQ}$ \\
\hline$<5$ & U & & $<\mathrm{EQ}$ \\
\hline$<54$ & JU & $Q$ & $<\mathrm{EQL}$ \\
\hline$<11$ & JU & $Q$ & $<\mathrm{EQ}$ \\
\hline$<10$ & U & & $<\mathrm{EQL}$ \\
\hline$<5$ & U & & $<\mathrm{EQL}$ \\
\hline$<5$ & U & & $<E Q$ \\
\hline$<5$ & $U$ & & $<E Q L$ \\
\hline$<5$ & $\mathrm{U}$ & & $<E Q L$ \\
\hline$<10$ & JU & $\mathbf{Q}$ & $<\mathrm{EQL}$ \\
\hline$<5$ & U & & $<\mathrm{EQ}$ \\
\hline$<5$ & $U$ & & $<E Q$ \\
\hline$<50$ & JU & $Q$ & $<E Q$ \\
\hline$<5$ & $U$ & & $<E Q$ \\
\hline$<11$ & JU & $Q$ & $<\mathrm{EQ}$ \\
\hline$<11$ & JU & $Q$ & $<E Q$ \\
\hline$<5$ & $\mathrm{U}$ & & $<\mathrm{EQ}$ \\
\hline$<11$ & JU & $Q$ & $<E Q$ \\
\hline$<11$ & JU & $Q$ & $<\mathrm{EQ}$ \\
\hline$<.2$ & $\mathrm{U}$ & $Q$ & $<\mathrm{EQ}$ \\
\hline$<.2$ & U & $Q$ & $<\mathrm{EQ}$ \\
\hline$<11$ & JU & $Q$ & $<\mathrm{EQ}$ \\
\hline$<27$ & JU & $Q$ & $<\mathrm{EQ}$ \\
\hline$<11$ & JU & $Q$ & $<E Q$ \\
\hline$<.2$ & $\mathrm{U}$ & $Q$ & < EQI \\
\hline$<11$ & JU & $Q$ & $<E Q$ \\
\hline$<27$ & JU & $Q$ & $<\mathrm{EQ}$ \\
\hline$<11$ & JU & $Q$ & $<\mathrm{EQ}$ \\
\hline$<25$ & JU & $Q$ & $<E Q$ \\
\hline$<11$ & JU & $Q$ & $<E Q$ \\
\hline$<11$ & JU & $Q$ & $<\mathrm{EQ}$ \\
\hline$<11$ & JU & $Q$ & $<\mathrm{EQ}$ \\
\hline$<11$ & JU & $Q$ & $<\mathrm{EQ}$ \\
\hline$<20$ & U & & $<E Q$ \\
\hline$<27$ & JU & $Q$ & $<\mathrm{EQ}$ \\
\hline$<11$ & JU & $Q$ & $<\mathrm{EQ}$ \\
\hline$<11$ & JU & $Q$ & $<E Q$ \\
\hline$<11$ & JU & $Q$ & $<\mathrm{EQ}$ \\
\hline$<.2$ & $U$ & $Q$ & $<\mathrm{EQ}$ \\
\hline$<11$ & JU & $Q$ & $<\mathrm{EQ}$ \\
\hline$<22$ & JU & $Q$ & $<\mathrm{EQ}$ \\
\hline$<11$ & JU & $Q$ & $<\mathrm{EQ}$ \\
\hline$<11$ & JU & $Q$ & $<E Q$ \\
\hline$<11$ & JU & $Q$ & $<\mathrm{EQ}$ \\
\hline$<11$ & JU & $a$ & $<\mathrm{EQ}$ \\
\hline
\end{tabular}

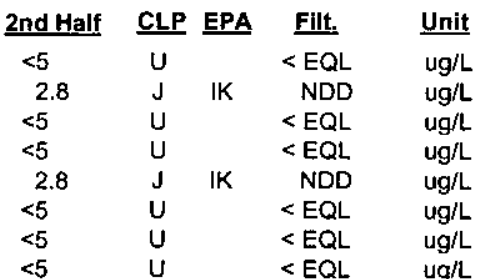

ugh

$\mathrm{ug} / \mathrm{L}$

$<10 \quad \mathrm{E} \quad<\mathrm{EQ} \quad \mathrm{ug} / \mathrm{L}$

$<5 \quad U \quad<E Q L \quad u g / L$

$<5 \quad U<\mathrm{EQL} \quad \mathrm{ug} / \mathrm{L}$

$<5 \quad U<\mathrm{EQL} \quad \mathrm{ug} / \mathrm{L}$

$<5 \quad U<E Q L \quad u g / L$

$<5 \quad U \quad \mathrm{HQ} / \mathrm{L}$

$<5 \quad U \quad<E Q L \quad u g / L$

$\mathrm{ug} / \mathrm{L}$

$\begin{array}{lll}\mathrm{U} & <\mathrm{EQL} & \mathrm{ug} / \mathrm{L} \\ \mathrm{ug} / \mathrm{L}\end{array}$

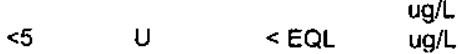

$u g / L$

ug/L

$\mathrm{ug} / \mathrm{L}$

$u g / L$

ug/L

ug/L

ug/L

ug/L

$u g / L$

ug/L

$\mathrm{ug} / \mathrm{L}$

$u g / L$

ugh

$\mathrm{ug} / \mathrm{L}$

$u g / L$

$u g / L$

ug/L

$u g / L$

$\mathrm{ug} / \mathrm{L}$

$\mathrm{ug} / \mathrm{L}$

ug/L.

$\mathrm{ug} / \mathrm{L}$

$\mathrm{ug} / \mathrm{L}$

$\mathrm{ug} / \mathrm{L}$

ug/L

ug/L

ug/L

Notes: Concentrations in bold italics exceed the groundwater protection standards listed in Appendix A. Bold italics were not assigned if the result was qualified with a "J", "R", "L", or "U" qualifier.

$+=$ exceeded the Groundwater Protection Standards listed in Appendix A. 
Table B. Assessment Monitoring Results for Groundwater Wells (Cont.)

WELL LFW 74D

ANALYTICAL DATA

Groundwater Protection Standard

ST Parameter

4-Chloroaniline

4-Chlorophenyl phenyl ether

4-Nitrophenol

5-Nitro-o-toluidine

7,12-Dimethylbenz(a)anthracene

Acenaphthene

Acenaphthylene

Acetone

Acetonitrile (Methyl cyanide)

Acetophenone

Acrolein

Acrylonitrile

Aldrin

Allyl chloride

Anthracene

Antimony, total recoverable

Arsenic, total recoverable

Barium, total recoverable

Benzene

Benzo(a)anthracene

Benzo(a)pyrene

Benzo(b)fluoranthene

Benzo( $g, h, i)$ perylene

Benzo(k)fluoranthene

Benzyl alcohol

Beryllium, total recoverable

Bis(2-chloroethoxy) methane

Bis(2-chloroethyl) ether

Bis(2-ethylhexyl) phthalate

Bromochloromethane

Bromodichloromethane

Bromoform

Bromomethane (Methyl bromide)

Butylbenzyl phthalate

Cadmium, total recoverable

Carbon disulfide

Carbon tetrachloride

Chlorobenzene

Chlorobenzilate

Chloroethane

Chloroethene (Vinyl chloride)

Chloroform

Chloromethane (Methyl chloride)

Chloroprene

Chromium, total recoverable

Chrysene

Cobalt, total recoverable

Copper, total recoverable

Cyanide

Di-n-butyl phthalate

Di-n-octyl phthalate

Diallate

Dibenz(a,h)anthracene

Dibenzofuran

Dibromochloromethane

Dibromomethane (Methylene bromide)

Dichlorodiftuoromethane

Dichloromethane (Methylene chloride)

Dieldrin

Diethyl phthalate

Dimethoate

Dimethyl phthalate

Diphenylamine

Disulfoton

Endosulfan I

Endosulfan If

Endosulfan sulfate

Endrin

$\frac{1 \text { st Half }}{<11} \frac{\text { CLP }}{J U} \frac{\text { EPA }}{\mathrm{F}}<\mathrm{EQ}$

$<11$ JU $Q<E Q L$

$<27$ JU $Q<E Q L$

$<11$ JU $Q<E Q$

$<11$ JU $Q<E Q L$

$<11$ JU $Q<E Q L$

$<11$ JU $Q<E Q L$

$\begin{array}{cccc}15 & \text { J } & \text { NDD } \\ <200 & \text { U } & & <E Q L\end{array}$

$<11$ JU $Q<E Q L$

$<50 \quad U<E Q$

$<10 \quad U<E Q$

$<.1 \quad U<E Q$

$<5 \quad U \quad<E Q L$

$<11$ JU $Q<E Q L$

$<43.2$ JU $\quad<E Q L$

$<10 \quad U \quad<E Q L$

$\begin{array}{llll}7.7 & J & \text { I } & \text { NDD } \\ <5 & U & & <E Q L\end{array}$

$<11$ JU $Q<E Q L$

$<11$ JU $Q<E Q L$

$<11$ JU $Q<E Q L$

$<11$ JU $Q<E Q$

$<11$ JU $Q<E Q L$

$<.12$ JU $<E Q L$

$<11$ JU $Q<E Q L$

$<11$ JU $Q<E Q L$

$<11$ JU $Q<E Q$

$<5 \quad U \quad<E Q L$

$<5 \quad U \quad<E Q L$

$<5 \quad U<E Q$

$<5 \quad U \quad<E Q L$

$<11$ JU $Q<E Q L$

$<10 \quad U<E Q$

$<5$

$<5$

$<5$

$<11$

$<5$

$<5$

$<20$

$<10$

$<11$

$<20$

$<20$

10

$<11$

$<11$

$<11$

$<11$

$<11$

$<5$

$<5$
$<5$

$<5$

$<.21$

$<11$

$<11$

$<11$

$<11$

$<11$

$<.1$

$<.21$

$<.21$

$<.21$

$<$ EQL

$<$ EQL

$<E Q L$

$Q<E Q L$

$<E Q L$

$<E Q L$

$<E Q L$

$<E Q L$

$<$ EQL

$<E Q L$

Q < EQL

$<$ EQL

$<$ EQL

$<$ EQL

$<E Q L$

$<E Q L$

$<E Q L$

$<E Q L$

$<$ EQL

$<E Q L$

$<$ EQL

NDD

$<$ EQL

$<$ EQL

$<$ EQL

$<E Q L$

$<E Q L$

$<E Q L$

$<E Q L$

$<E Q L$

$<E Q L$

$<$ EQL 2nd Half CLP EPA Filt. Unit

\begin{tabular}{|c|c|c|c|}
\hline $\begin{array}{c}89 \\
<200\end{array}$ & $\begin{array}{l}\mathrm{J} \\
\mathrm{U}\end{array}$ & $\mathrm{K}$ & $\begin{aligned} & N D D \\
< & \mathrm{EQL}\end{aligned}$ \\
\hline$<50$ & $U$ & & $<E Q L$ \\
\hline$<10$ & $\mathbf{U}$ & & $<E Q L$ \\
\hline$<5$ & $\mathrm{U}$ & & $<E Q L$ \\
\hline$<100$ & $\mathrm{U}$ & & $<E Q L$ \\
\hline$<10$ & $\mathrm{U}$ & & $<E Q L$ \\
\hline 5.57 & $\mathrm{~J}$ & 1 & NDD \\
\hline$<5$ & $\mathrm{U}$ & & $<\mathrm{EQL}$ \\
\hline
\end{tabular}

$u g / L$

$\mathrm{ug} / \mathrm{L}$

ug/L

ugil

$\mathrm{ug} / \mathrm{L}$

ug/L

$\mathrm{ug} / \mathrm{h}$

$\mathrm{ug} / \mathrm{L}$

$u g / L$

$\mathrm{ug} / \mathrm{L}$

$\mathrm{ug} / \mathrm{L}$

$u g / L$

$u g / L$

ugh

$\mathrm{ug} / \mathrm{L}$

$\mathrm{ug} / \mathrm{L}$

$u g / L$

$u g / L$

$\mathrm{ug} / \mathrm{L}$

$u g / L$

$<.25$ JU $<$ EQL Ug

$\mathrm{ug} / \mathrm{L}$

ug/L

$\mathrm{ug} / \mathrm{L}$

ug/t

$\mathrm{ug} / \mathrm{L}$

$\mathrm{ug} / \mathrm{L}$

ug/h

ug/L

Q Rejected

$\mathrm{ug} / \mathrm{L}$

ug/L

$\mathrm{ug} / \mathrm{L}$

ug/L

ug/L

$\mathrm{ug} / \mathrm{L}$

ug/L

$\mathrm{ug} / \mathrm{L}$

ug/

ug/L

$u g / L$

$\mathrm{ug} / \mathrm{L}$

ugh

$u g / L$

ug/ $/$

$u g / L$

ug/

ugh

ugh

$<5 \quad \mathrm{U} \quad<\mathrm{EQL} \quad \mathrm{ug} / \mathrm{L}$

$<$ EQL Ug/L

$<2.8 \quad U \quad<E Q L \quad u g / L$

$\mathrm{ug} / \mathrm{L}$

ugh

$\mathrm{ug} / \mathrm{h}$

ug/h

ugh

ugh

ugh

ugl

$\mathrm{ug} / \mathrm{L}$

$\mathrm{ug} / \mathrm{L}$

Notes: Concentrations in bold italics exceed the groundwater protection standards listed in Appendix A. Bold italics were not assigned if the result was qualified with a "J", "R", "L", or "U" qualifier.

$+=$ exceeded the Groundwater Protection Standards listed in Appendix A. 
Table B. Assessment Monitoring Results for Groundwater Wells (Cont.)

WELL LFW 74D

ANALYTICAL DATA

Groundwater Protection Standard

\section{ST Parameter}

Endrin aldehyde

Ethyl methacrylate

Ethyl methanesulfonate

Ethylbenzene

Famphur

Fiuoranthene

Fluorene

Heptachlor

Heptachlor epoxide

Hexachlorobenzene

Hexachlorobutadiene

Hexachlorocyclopentadiene

Hexachloroethane

Hexachloropropene

Indeno(1,2,3-c,d)pyrene

lodomethane (Methyl iodide)

Isobutyl alcohol

Isodrin

Isophorone

Isosafrole

Kepone

Lead, total recoverable

Lindane

Mercury, total recoverable

Methacrylonitrile

Methapyrilene

Methoxychlor

Methyl ethyl ketone

Methyl isobutyl ketone

Methyl methacrylate

Methyl methanesulfonate

$\mathrm{N}$-Nitrosodi-n-butylamine

$\mathrm{N}$-Nitrosodiethylamine

N-Nitrosodimethylamine

N-Nitrosodiphenylamine

$\mathrm{N}$-Nitrosodipropylamine

$\mathrm{N}$-Nitrosomethylethylamine

$\mathrm{N}$-Nitrosopiperidine

$\mathrm{N}$-Nitrosopyrrolidine

Naphthalene

Nickel, total recoverable

Nitrobenzene

PCB

PCB 1016

PCB 1232

PCB 1242

PCB 1248

PCB 1254

PCB 1260

Parathion

Parathion methyl

Pentachlorobenzene

Pentachloronitrobenzene

Pentachlorophenol

Phenacetin

Phenanthrene

Phenol

Phorate

Pronamid

Propionitrile

Pyrene

Safrole

Selenium, total recoverable

Silver, total recoverable

$+\quad$ Specific conductance

Styrene

Sulfide
O,O,O-Triethyl phosphorothioate

\begin{tabular}{|c|c|c|c|}
\hline Ist Half & CLP & EPA & Filt. \\
\hline$<.21$ & $u$ & & $<E Q L$ \\
\hline$<5$ & $U$ & & $<E Q L$ \\
\hline$<11$ & JU & $Q$ & $<\mathrm{EQL}$ \\
\hline$<5$ & U & & $<E Q L$ \\
\hline$<200$ & JU & $Q$ & $<\mathrm{EQL}$ \\
\hline$<11$ & JU & $Q$ & $<E Q L$ \\
\hline$<11$ & JU & $Q$ & $<\mathrm{EQL}$ \\
\hline$<.1$ & $u$ & & $<E Q L$ \\
\hline$<.1$ & $u$ & & $<E Q L$ \\
\hline$<11$ & JU & $\mathbf{Q}$ & $<E Q L$ \\
\hline$<11$ & JU & $Q$ & $<\mathrm{EQL}$ \\
\hline$<11$ & JU & $Q$ & $<\mathrm{EQL}$ \\
\hline$<11$ & JU & $Q$ & $<E Q L$ \\
\hline$<54$ & JU & $\mathbf{Q}$ & $<E Q L$ \\
\hline$<11$ & JU & $Q$ & $<E Q L$ \\
\hline$<5$ & U & & $<E Q L$ \\
\hline$<500$ & U & & $<E Q L$ \\
\hline$<11$ & JU & $Q$ & $<E Q L$ \\
\hline$<11$ & JU & $Q$ & $<E Q L$ \\
\hline$<11$ & JU & $Q$ & $<\mathrm{EQL}$ \\
\hline$<11$ & JU & $Q$ & $<E Q L$ \\
\hline$<10$ & $u$ & & $<$ EQL \\
\hline$<.1$ & U & & $<\mathrm{EQL}$ \\
\hline .742 & & & $<2$ \\
\hline$<200$ & $U$ & & $<E Q L$ \\
\hline$<11$ & JU & $Q$ & $<E Q L$ \\
\hline$<1$ & u & & $<E Q L$ \\
\hline$<20$ & $u$ & & $<E Q L$ \\
\hline$<10$ & U & & $<E Q L$ \\
\hline$<20$ & u & & $<\mathrm{EQL}$ \\
\hline$<11$ & JU & $Q$ & $<E Q L$ \\
\hline$<11$ & JU & $Q$ & $<\mathrm{EQL}$ \\
\hline$<11$ & JU & $Q$ & $<\mathrm{EQL}$ \\
\hline$<27$ & JU & $Q$ & $<\mathrm{EQL}$ \\
\hline$<11$ & JU & $Q$ & $<E Q L$ \\
\hline$<11$ & JU & $Q$ & $<\mathrm{EQL}$ \\
\hline$<11$ & JU & $Q$ & $<\mathrm{EQL}$ \\
\hline$<11$ & JU & $Q$ & $<\mathrm{EQL}$ \\
\hline$<11$ & JU & $Q$ & $<E Q L$ \\
\hline$<11$ & JU & $Q$ & $<\mathrm{EQL}$ \\
\hline$<6.34$ & JU & & $<E Q L$ \\
\hline$<11$ & JU & $Q$ & $<\mathrm{EQL}$ \\
\hline$<11$ & JU & $Q$ & $<E Q L$ \\
\hline$<1$ & U & & $<\mathrm{EQL}$ \\
\hline$<1$ & $u$ & & $<E Q L$ \\
\hline$<2.1$ & U & & $<E Q L$ \\
\hline$<1$ & $u$ & & $<E Q L$ \\
\hline$<1$ & U & & $<\mathrm{EQL}$ \\
\hline$<1$ & $U$ & & $<\mathrm{EQL}$ \\
\hline$<11$ & JU & $Q$ & $<E Q L$ \\
\hline$<11$ & JU & $Q$ & $<E Q L$ \\
\hline$<54$ & JU & $Q$ & $<\mathrm{EQL}$ \\
\hline$<11$ & JU & $Q$ & $<E Q L$ \\
\hline$<27$ & JU & $Q$ & $<\mathrm{EQL}$ \\
\hline$<11$ & JU & $Q$ & $<E Q L$ \\
\hline$<11$ & JU & $Q$ & $<E Q L$ \\
\hline$<11$ & JU & $Q$ & $<E Q L$ \\
\hline$<11$ & JU & $Q$ & $<\mathrm{EQL}$ \\
\hline$<11$ & JU & $Q$ & $<\mathrm{EQL}$ \\
\hline$<200$ & U & & $<E Q L$ \\
\hline$<11$ & JU & $Q$ & $<E Q L$ \\
\hline$<11$ & JU & $Q$ & $<E Q L$ \\
\hline$<10$ & $u$ & & $<E Q L$ \\
\hline$<20$ & $U$ & & $<E Q L$ \\
\hline 20.3 & & & $<37$ \\
\hline$<5$ & U & & $<\mathrm{EQL}$ \\
\hline 1000 & $\mathrm{R}$ & $Q$ & Rejected \\
\hline
\end{tabular}

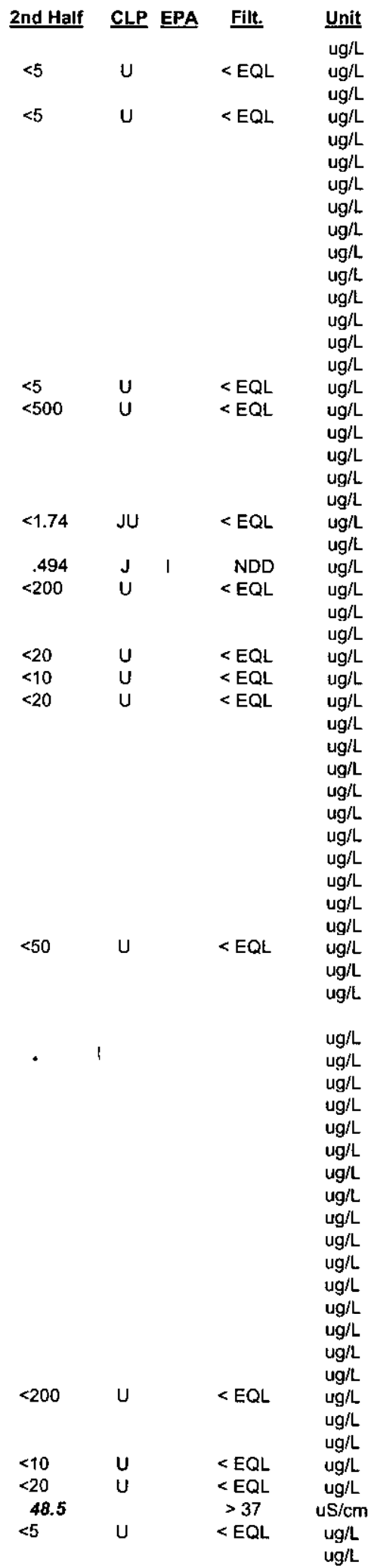

Notes: Concentrations in bold italics exceed the groundwater protection standards listed in Appendix $A$. Bold italics were not assigned if the result was qualified with a "J", "R", "L", or "U" qualifier.

$+=$ exceeded the Groundwater Protection Standards listed in Appendix A. 
Table B. Assessment Monitoring Results for Groundwater Wells (Cont.)

WELL LFW 74D

ANALYTICAL DATA

Groundwater Protection Standard

ST Parameter

Tetrachloroethylene

Thallium, total recoverable

Thionazin

Tin, total recoverable

Toluene

Toxaphene

Trichloroethylene

Trichlorofluoromethane

Vanadium, total recoverable

Vinyl acetate

Xylenes

Zinc, total recoverable

alpha-Benzene hexachloride alpha-Chlordane

beta-Benzene hexachloride

cis-1,2-Dichloroethylene

cis-1,3-Dichloropropene

delta-Benzene hexachloride

gamma-Chlordane

m-Cresol (3-Methylphenol)

$\mathrm{m}-\mathrm{Nitroaniline}$

o-Cresol (2-Methylphenol)

o-Nitroaniline

0 -Toluidine

p.p"-DDD

$p, p^{\prime \prime}-D D E$

$p, p^{\prime \prime}-D D T$

p-Cresol (4-Methylphenol)

p-Dimethylaminoazobenzene

p-Nitroaniline

p-Phenylenediamine

$\mathrm{pH}$

trans-1,2-Dichloroethylene

trans-1,3-Dichloropropene

trans-1,4-Dichloro-2-butene

\begin{tabular}{|c|c|c|c|}
\hline 1st Half & CLP & EPA & Filt. \\
\hline$<5$ & $U$ & & $<E Q L$ \\
\hline$<10$ & U & & $<$ EQL \\
\hline$<11$ & JU & $Q$ & < EQL \\
\hline$<200$ & U & & $<E Q L$ \\
\hline$<5$ & $U$ & & $<E Q L$ \\
\hline$<2.1$ & U & & $<$ EQL \\
\hline$<5$ & U & & $<E Q L$ \\
\hline 21 & & & $>20$ \\
\hline$<10$ & U & & $<\mathrm{EQL}$ \\
\hline$<5$ & U & & $<E Q L$ \\
\hline$<10$ & U & & $<E Q L$ \\
\hline 23.8 & & & $<29.3$ \\
\hline$<.1$ & U & & $<$ EQL \\
\hline$<.1$ & $u$ & & $<E Q L$ \\
\hline$<.1$ & U & & $<\mathrm{EQL}$ \\
\hline$<5$ & U & & $<E Q L$ \\
\hline$<5$ & U & & $<\mathrm{EQL}$ \\
\hline$<.1$ & U & & $<\mathrm{EQL}$ \\
\hline$<.1$ & U & & $<\mathrm{EQL}$ \\
\hline$<27$ & JU & $Q$ & $<E Q L$ \\
\hline$<11$ & JU & $Q$ & $<E Q L$ \\
\hline$<27$ & JU & $Q$ & $<$ EQL \\
\hline$<11$ & JU & $Q$ & $<E Q L$ \\
\hline$<.21$ & U & & $<E Q L$ \\
\hline$<.21$ & U & & $<E Q L$ \\
\hline$<.21$ & U & & $<E Q L$ \\
\hline$<10$ & JU & $Q$ & $<E Q L$ \\
\hline$<11$ & JU & $Q$ & $<\mathrm{EQL}$ \\
\hline$<11$ & JU & $\mathbf{Q}$ & $<E Q L$ \\
\hline$<11$ & JU & $Q$ & $<E Q L$ \\
\hline 5.8 & $\mathrm{~J}$ & $Q$ & NDD \\
\hline$<5$ & U & & $<$ EQL \\
\hline$<5$ & U & & $<E Q L$ \\
\hline$<20$ & $U$ & & $<\mathrm{EQL}$ \\
\hline
\end{tabular}

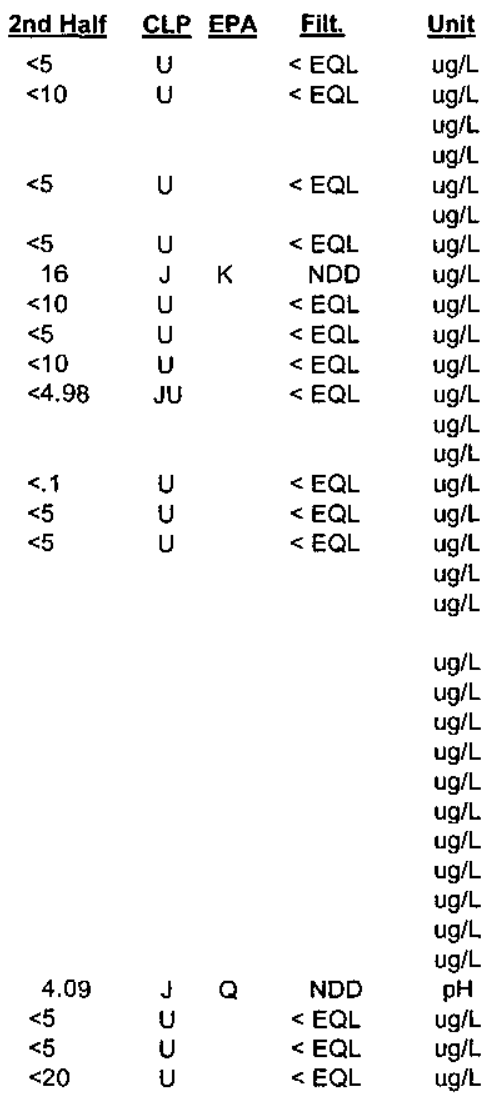

Notes: Concentrations in bold italics exceed the groundwater protection standards listed in Appendix A. Bold italics were not assigned if the result was qualified with a "J", "R", "L", or "U" qualifier.

$+=$ exceeded the Groundwater Protection Standards listed in Appendix A. 
Table B. Assessment Monitoring Results for Groundwater Wells (Cont.)

WELL LFW 75C

$\begin{array}{ll}\text { SRS Coord. } & \text { LatiLongitude } \\ \text { N } 85856.8 & 33.28966 \text { Deg N } \\ \text { E 45357 } & -81.7141 \text { Deg } W\end{array}$

SAMPLE DATE FIELD DATA

\section{Parameter \\ Water Elevation \\ $\mathrm{pH}$ \\ Sp. Conductance \\ Water temperature \\ Alkalinity as $\mathrm{CaCO} 3$ \\ Turbidity \\ Volumes purged \\ Sampling code}

\section{ANALYTICAL DATA}

Groundwater Protection Standard ST Parameter

1,1,1,2-Tetrachloroethane

1,1,1-Trichloroethane

1,1,2,2-Tetrachioroethane

1,1,2-Trichloroethane

1,1-Dichloroethane

1,1-Dichloroethylene

1,1-Dichloropropene

1,2,3-Trichloropropane

1,2,4,5-Tetrachlorobenzene

1,2,4-Trichlorobenzene

1,2-Dibromo-3-chloropropane

1,2-Dibromoethane

1,2-Dichlorobenzene

1,2-Dichloroethane

1,2-Dichloropropane

1,3,5-Trinitrobenzene

1,3-Dichlorobenzene

1,3-Dichloropropane

1,3-Dinitrobenzene

1,4-Dichlorobenzene

1,4-Naphthoquinone

1-Naphthylamine

2,2-Dichloropropane

2,2-Oxybis(1-chloropropane)

2,3,4,6-Tetrachlorophenol

2,4,5-T

2,4,5-TP (Silvex)

2,4,5-Trichlorophenol

2,4,6-Trichlorophenol

2,4-Dichlorophenol

2,4-Dichlorophenoxyacetic acid

2,4-Dimethyl phenol

2,4-Dinitrophenol

2,4-Dinitrotoluene

2,6-Dichlorophenol

2,6-Dinitrotoluene

2-Acetylaminofluorene

2-Chloronaphthalene

2-Chlorophenol

2-Hexanone

2-Methyl-4,6-dinitropheno

2-Methyinaphthalene

2-Naphthylamine

2-Nitrophenol

2-sec-Butyl-4,6-dinitrophenol

3,3"-Dichlorobenzidine

3,3"-Dimethylbenzidine

3-Methylcholanthrene

4-Aminobiphenyl

4-Bromophenyl phenyl ether

4-Chloro-m-cresol

\author{
Screen Zone Elevation \\ $115.6-100.6 \mathrm{ft} \mathrm{msl}$
}

$03 / 08 / 00$

\begin{tabular}{l} 
1st Half \\
\hline 160.7 \\
4.9 \\
31 \\
19.6 \\
2 \\
.6 \\
3.87
\end{tabular}

1st Half

$<5$

$<5$

$<5$
$<5$

$<5$

$<5$

$<5$

$<53$

$<11$

$<10$

$<5$

$<5$

$<5$

$<5$

$<10$

$<5$
$<5$

$<5$
$<50$

$<5$

$<11$

$<11$

$<5$

$<11$

$<11$

$<.2$

$<.2$

$<11$

$<26$

$<11$

$<.2$

$<11$

$<26$

$<11$

$<25$

$<11$

$<11$

$<11$

$<11$

$<20$

$<26$

$<11$

$<11$

$<11$

$<.2$

$<11$

$<21$

$<11$

$<11$

$<11$

$<11$

\author{
Top of Casing \\ $197.8 \mathrm{ft} \mathrm{ms}$
}

$\frac{\text { Casing }}{4^{n} \mathrm{PVC}}$

Pumn

Screen Zone

$10 / 02 / 00$

2nd Half
160.23
4.9
32
20.6
0
.8
3.95

Unit

ft msi

$\mathrm{pH}$

uS $/ \mathrm{cm}$

deg. C

NG/L

well volume
Middle

Notes: Concentrations in bold italics exceed the groundwater protection standards listed in Appendix A. Bold italics were not assigned if the result was qualified with a "J", "R", "L", or "U" qualifier.

$+=$ exceeded the Groundwater Protection Standards listed in Appendix A.

2ndHalf CLP EPA Filt. Un

$\begin{array}{llll}<5 & U & <E Q L & u g / \\ <5 & U & <E Q L & u g / \\ <5 & U & <E Q L & u g / \\ <5 & U & <E Q L & u g / \\ <5 & U & <E Q L & u g / \\ <5 & U & <E Q L & u g / \\ <5 & U & <E Q L & u g / \\ <5 & U & <E Q L & u g\end{array}$

$<10 \quad U \quad<E Q L \quad \mathrm{ug} / \mathrm{L}$

$<5 \quad U \quad<E Q \quad \mathrm{ug} / \mathrm{L}$

$<5 \quad \mathrm{U} \quad<\mathrm{EQL} \quad \mathrm{ug} / \mathrm{L}$

$<5 \quad U \quad<$ EQL ug/L

$<5 \quad \mathrm{U} \quad<\mathrm{EQL} \quad \mathrm{ug} / \mathrm{L}$

Ug/L

$\begin{array}{llll}<5 & U & <E Q L & \mathrm{ug} / \mathrm{L} \\ <5 & \mathrm{E} & <\mathrm{EQL} & \mathrm{ug} / \mathrm{L}\end{array}$

u ug/L

$\begin{array}{ll}\mathrm{u} & \mathrm{ug} / \mathrm{L} \\ \mathrm{ug} / \mathrm{L}\end{array}$

$\begin{array}{lllll}<E Q L & <5 & \mathrm{E} & <\mathrm{EQL} & \mathrm{ug} / \mathrm{L} \\ \mathrm{ug} / \mathrm{L}\end{array}$

$<E Q L$

$<E Q L$

$<E Q L$

$<E Q L$

$<E Q L$

$<E Q L$

$<E Q L$

$<$ EQL

$<E Q L$

$<\mathrm{EQL}$

$<E Q L$

$<$ EQL

$<E Q L$

$<$ EQL

$<E Q L$

$<E Q L$

$<E Q L$

$<E Q L$

$<$ EQL

$<E Q L$

$<E Q L$

$<E Q L$

$<E Q L$

$<E Q L$

$<E Q L$

$<\mathrm{EQL}$

Unit

$g / h$

$\operatorname{ug} / \mathrm{L}$ $g / L$ $g / L$ $g / L$ L

L

$\mathrm{ug} / \mathrm{L}$

ug/L

$u g / h$

$u g / L$

$u g / L$

$\mathrm{ug} / \mathrm{L}$

ug/L

$u g / L$

ug/L

$\mathrm{ug} / \mathrm{L}$

$\mathrm{ug} / \mathrm{L}$

ug/L

ug/L

$<20 \quad U \quad<$ EQL $\quad \mathrm{ug} / \mathrm{L}$

ug/L

ug/L

$\mathrm{ug} / \mathrm{L}$

ug/L

ug/L

ug $/ L$

$\mathrm{ug} / \mathrm{L}$

ug/L

ug/L

ug/L

$\mathrm{ug} / \mathrm{L}$ 
ANALYTICAL DATA

Groundwater Protection Standard

ST Parameter

4-Chloroaniline

4-Chlorophenyl phenyl ether.

4-Nitrophenol

5-Nitro-o-toluidine

7,12-Dimethylbenz(a)anthracene

Acenaphthene

Acenaphthylene

Acetone

Acetonitrile (Methyl cyanide)

Acetophenone

Acrolein

Acrylonitrile

Aldrin

Allyl chloride

Anthracene

Antimony, total recoverable

Arsenic, total recoverable

Barium, total recoverable

Benzene

Benzo(a)anthracene

Benzo(a)pyrene

Benzo(b)fluoranthene

Benzo(g,h,i)perylene

Benzo(k)fluoranthene

Benzyl alcohol

Beryllium, total recoverable

Bis(2-chloroethoxy) methane

Bis(2-chloroethyl) ether

Bis(2-ethylhexyl) phthalate

Bromochloromethane

Bromodichloromethane

Bromoform

Bromomethane (Methyl bromide)

Butylbenzyl phthalate

Cadmium, total recoverable

Carbon disulfide

Carbon tetrachloride

Chlorobenzene

Chlorobenzilate

Chloroethane

Chioroethene (Vinyl chloride)

Chloroform

Chloromethane (Methyl chloride)

Chloroprene

Chromium, total recoverable

Chrysene

Cobalt, total recoverable

Copper, total recoverable

Cyanide

Di-n-butyl phthalate

Di-n-octyl phthalate

Diallate

Dibenz $(a, h)$ anthracene

Dibenzofuran

Dibromochloromethane

Dibromomethane (Methylene bromide)

Dichiorodifluoromethane

Dichloromethane (Methylene chloride)

Dieldrin

Diethyl phthalate

Dimethoate

Dimethyl phthalate

Diphenylamine

Disulfoton

Endosulfan I

Endosulfan II

Endosulfan sulfate

$\frac{1 \text { st Half }}{<11} \frac{\text { CLP }}{\mathrm{JU}} \underset{\mathrm{EPA}}{<\mathrm{Filt}}$

$<11$ JU $Q<E Q L$

$<26$ JU $Q<E Q L$

$<11$ JU $Q<E Q L$

$<11$ JU $Q<E Q$

$<11$ JU $Q<E Q L$

$<11$ JU $Q<E Q L$

$<20 \quad \mathrm{E}<\mathrm{EQL}$

$<200 \quad U<E Q L$

$<11$ JU $Q<E Q L$

$<50 \quad U<E Q L$

$<10 \quad U<E Q$

$<1 \quad U \quad<E Q L$

$<5 \quad U<E Q L$

$<11$ JU $Q<E Q L$

$<100 \quad U<E Q L$

$<10 \quad U<E Q$

$\begin{array}{llll}6.74 & \mathrm{~J} & \mathrm{~N} & \mathrm{NDD} \\ <5 & \mathrm{U} & & <\mathrm{EQL}\end{array}$

$<11$ JU $Q<E Q$

$<11$ JU $Q<E Q$

$<11$ JU $Q<E Q L$

$<11$ JU $Q<E Q$

$<11$ JU $Q<E Q L$

$<.16$ JU $<$ EQL

$<11$ JU $Q<E Q L$

$<11$ JU $Q<E Q L$

$<11$ JU $Q<E Q L$

$<5 \quad \mathrm{O}<\mathrm{EQL}$

$<5 \quad U<E Q$

$<5 \quad U \quad<E Q L$

$<5 \quad U<E Q L$

$<11$ JU $Q<E Q L$

$<10 \quad U<$ LQL

$<5 \quad U \quad<E Q L$

$<5 \quad U<E Q$

$<5 \quad U \quad<E Q L$

$<11$ JU $Q<E Q L$

$<5 \quad U<E Q$

$<5 \quad U<E Q L$

$<5 \quad \mathrm{U}<\mathrm{EQL}$

$<5 \quad U<E Q L$

$<20 \quad U<E Q$

$<10 \quad U \quad<E Q$

$<11$ JU $Q \quad<E Q L$

$<20 \quad U \quad<E Q L$

$\begin{array}{cccc}<2.17 & \mathrm{JU} & & <\mathrm{EQL} \\ 10 & \mathrm{R} & \mathrm{Q} & \text { Rejected }\end{array}$

$<11$ JU $Q<$ EQL

$<11$ JU $Q<E Q L$

$<11$ JU $Q<E Q$

$<11$ JU $Q<E Q L$

$<11$ JU $Q<E Q L$

$<5 \quad U \quad<E Q L$

$<5 \quad U<E Q$

$<5 \quad U<E Q$

19 J I NDD

$<.21 \quad U<E Q L$

$<11$ JU $Q<E Q$

$<11$ JU Q <EQI

$<11$ JU $Q<E Q L$

$<11$ JU $Q<E Q$

$<11$ JU $Q<E Q t$

$<1 \quad U<E Q L$

$<.21 \quad U \quad<E Q L$

$<.21 \quad U<E Q L$

$<.21 \quad U<E Q$ 2nd Half CLP EPA Filt. Unit

$\mathrm{ug} / \mathrm{L}$

ug/

$\mathrm{ug} / \mathrm{L}$

ug/L

ug $/ L$

$\mathrm{ug} / \mathrm{L}$

$\mathrm{ug} / \mathrm{L}$

$\mathrm{ug} / \mathrm{L}$

$u g / h$

ug/h

ug/h

$\mathrm{ug} / \mathrm{L}$

$\mathrm{ug} / \mathrm{L}$

ugh

ug/L

ug/L

ug/L

ug/L

$\mathrm{ug} / \mathrm{h}$

ug/L.

$\mathrm{ug} / \mathrm{L}$

$\mathrm{ug} / \mathrm{L}$

$<.32$ JU $<$ EQL ug/l

$\mathrm{ug} / \mathrm{L}$

ug/h

ug/L

$\begin{array}{llll}<5 & U & <E Q L & u g / h \\ <5 & U & <E Q L & u g / h\end{array}$

$<5 \quad \mathrm{U} \quad<\mathrm{EQL} \quad \mathrm{ug} / \mathrm{h}$

$<5 \quad U<$ EQL ug/L

$<10 \quad U \quad<E Q L \quad \mathrm{ug} / \mathrm{h}$

$<5 \quad U \quad<E Q L$

$<5 \quad 4$

$<$ EQL

$\mathrm{ug} / \mathrm{L}$

ug/h

$\mathrm{ug} / \mathrm{L}$

$\mathrm{ug} / \mathrm{L}$

$\begin{array}{llll}<5 & \mathrm{U} & <\mathrm{EQL} & \mathrm{ug} / \mathrm{L} \\ <5 & \mathrm{U} & <\mathrm{EQL} & \mathrm{ug} / \mathrm{L}\end{array}$

$<5 \quad U \quad<E Q L \quad u g / L$

$<5 \quad U<E Q$

$<20 \quad U<E O L$

$<10 \quad U<E Q$

$8.34 \mathrm{~J} / \mathrm{ug} / \mathrm{L}$

$<9.7 \quad \mathrm{ug} / \mathrm{L}$

$\mathrm{ug} / \mathrm{L}$

$u g / L$

$u g / L$

$u g / L$

$\mathrm{ug} / \mathrm{L}$

$\mathrm{ug} / \mathrm{L}$

\section{$<5$}

$<5$

$<10$

$\mathrm{ug} / \mathrm{L}$

ull

$\mathrm{ug} / \mathrm{L}$

$\mathrm{ug} / \mathrm{L}$

$u g / L$

ug/L

$\mathrm{ug} / \mathrm{L}$

$u g / L$

ug/L

ug/L

$\mathrm{ug} / \mathrm{L}$

$\mathrm{ug} / \mathrm{L}$

Notes: Concentrations in bold italics exceed the groundwater protection standards listed in Appendix A. Bold italics were not assigned if the result was qualified with a "J", "R", "L", or "U" qualifier.

$+=$ exceeded the Groundwater Protection Standards listed in Appendix A. 
Table B. Assessment Monitoring Results for Groundwater Wells (Cont.)

WELL LFW 75C

ANALYTICAL DATA

Groundwater Protection Standard

ST Parameter

Ethyl methacrylate

Ethyl methanesulfonate

Famphur

Fluoranthene

Fluorene

Heptachlor

Heptachlor epoxide

Hexachlorobenzene

Hexachlorobutadiene

Hexachlorocyclopentadiene

Hexachloroethane

Hexachloropropene

Indeno(1,2,3-c,d)pyrene

lodomethane (Methyl iodide)

Isobutyl alcohol

Isodrin

Isophorone

Isosafrole

Kepone

Lead, total recoverable

Lindane

Mercury, total recoverable

Methacrylonitrile

Methapyrilene

Methoxychlor

Methyl ethyl ketone

Methyl isobutyl ketone

Methyl methacrylate

Methyl methanesulfonate

N-Nitrosodi-n-butylamine

$\mathrm{N}$-Nitrosodiethylamine

$\mathrm{N}$-Nitrosodimethylamine

$\mathrm{N}$-Nitrosodiphenylamine

$\mathrm{N}$-Nitrosodipropylamine

$\mathrm{N}$-Nitrosomethylethylamine

$\mathrm{N}$-Nitrosopiperidine

$\mathrm{N}$-Nitrosopyrrolidine

Naphthalene

Nickel, total recoverable

Nitrobenzene

$0,0,0$-Triethyl phosphorothioate

PCB

PCB 1016

PCB 1232

PCB 1242

PCB 1248

PCB 1254

PCB 1260

Parathion

Parathion methyl

Pentachlorobenzene

Pentachloronitrobenzene

Pentachlorophenol

Phenacetin

Phenanthrene

Phenol

Phorate

Pronamid

Propionitrile

Pyrene

Safrole

Selenium, total recoverable

Silver, total recoverable

Specific conductance

Styrene

Sulfide
Endrin aldehyde

Ethylbenzene

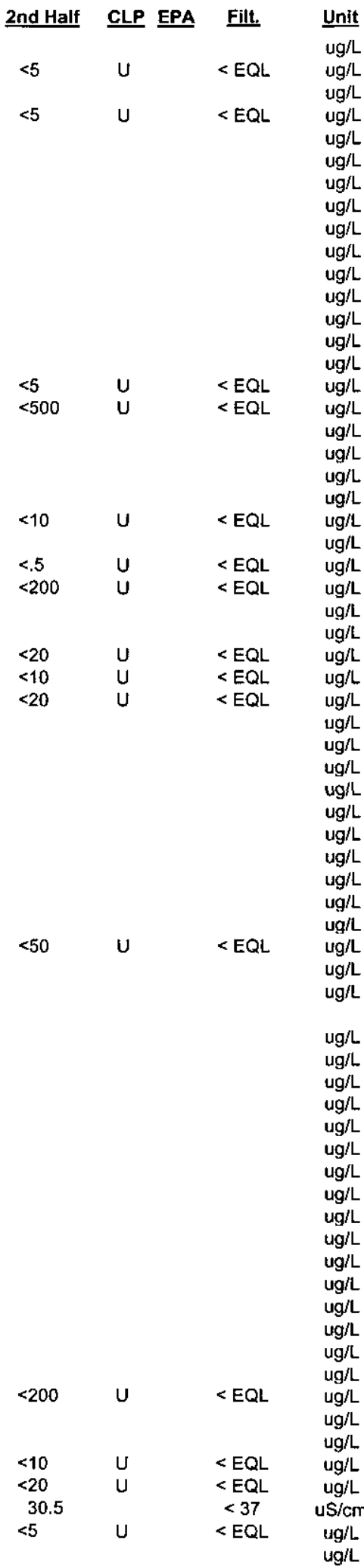

Notes: Concentrations in bold italics exceed the groundwater protection standards listed in Appendix A. Bold italics were not assigned if the result was qualified with a "J", "R", "L", or "U" qualifier.

$+=$ exceeded the Groundwater Protection Standards listed in Appendix A. 
Table B. Assessment Monitoring Results for Groundwater Wells (Cont.)

WELL LFW 75C

ANALYTICAL DATA

Groundwater Protection Standard

ST Parameter

Tetrachloroethylene

Thallium, total recoverable

Thionazin

Tin, total recoverable

Toluene

Toxaphene

Trichloroethylene

Trichlorofluoromethane

Vanadium, total recoverable

Vinyl acetate

Xylenes

Zinc, total recoverable

alpha-Benzene hexachloride

alpha-Chlordane

beta-Benzene hexachloride

cis-1,2-Dichloroethylene

cis-1,3-Dichloropropene

delta-Benzene hexachloride

gamma-Chlordane

m-Cresol (3-Methylphenol)

$\mathrm{m}$-Nitroaniline

o-Cresol (2-Methylphenol)

o-Nitroanitine

o-Toluidine

$p, p^{\prime \prime-D D D ~}$

$p, p "-D D E$

$p, p "-D D T$

p-Cresol (4-Methylphenol)

p-Dimethylaminoazobenzene

p-Nitroaniiine

p-Phenylenediamine

$\mathrm{pH}$

trans-1,2-Dichloroethylene

trans-1,3-Dichloropropene

trans-1,4-Dichloro-2-butene

\begin{tabular}{|c|c|c|c|}
\hline 1st Half & CLP & EPA & Filt. \\
\hline$<5$ & $U$ & & $<E Q L$ \\
\hline$<5.04$ & -JU & & $<$ EQL \\
\hline$<11$ & JU & $Q$ & $<E Q L$ \\
\hline$<200$ & $U$ & & $<$ EQL \\
\hline$<5$ & $\mathrm{U}$ & & $<$ EQL \\
\hline$<2.1$ & U & & $<$ EQL \\
\hline$<5$ & $U$ & & $<E Q L$ \\
\hline$<5$ & $U$ & & $<E Q L$ \\
\hline$<10$ & $U$ & & $<E Q L$ \\
\hline$<5$ & $\mathrm{U}$ & & $<E Q L$ \\
\hline$<10$ & U & & $<E Q L$ \\
\hline 20.2 & & & $<29.3$ \\
\hline$<.1$ & U & & $<\mathrm{EQL}$ \\
\hline$<.1$ & U & & $<\mathrm{EQL}$ \\
\hline$<.1$ & U & & $<\mathrm{EQL}$ \\
\hline$<5$ & U & & $<\mathrm{EQL}$ \\
\hline$<5$ & U & & $<$ EQL \\
\hline$<.1$ & U & & $<$ EQL \\
\hline$<.1$ & U & & $<$ EQL \\
\hline$<26$ & JU & $Q$ & $<E Q L$ \\
\hline$<11$ & JU & $Q$ & $<\mathrm{EQL}$ \\
\hline$<26$ & JU & $Q$ & $<E Q L$ \\
\hline$<11$ & JU & $Q$ & $<$ EQL \\
\hline$<.21$ & U & & $<E Q L$ \\
\hline$<.21$ & U & & $<E Q L$ \\
\hline$<.21$ & U & & $<E Q L$ \\
\hline$<10$ & JU & $Q$ & $<E Q L$ \\
\hline$<11$ & JU & $\mathbf{Q}$ & $<E Q L$ \\
\hline$<11$ & JU & Q. & $<E Q L$ \\
\hline$<11$ & JU & $Q$ & $<E Q L$ \\
\hline 5.5 & J & $Q$ & NDD \\
\hline$<5$ & U & & $<$ EQL \\
\hline$<5$ & U & & $<$ EQL \\
\hline$<20$ & U & & $<E Q L$ \\
\hline
\end{tabular}

\begin{tabular}{|c|c|c|c|c|}
\hline 2nd Half & CLP & EPA & Filt. & Unit \\
\hline$<5$ & U & & $<\mathrm{EQL}$ & ug/L \\
\hline$<10$ & $U$ & & $<E Q L$ & ug/L \\
\hline & & & & $\mathrm{ug} / \mathrm{L}$ \\
\hline$<5$ & U & & $<\mathrm{EQL}$ & ug $/ \mathrm{L}$ \\
\hline$<5$ & 11 & & $<\mathrm{FOl}$ & ugh \\
\hline$<5$ & u & & $<\mathrm{EQL}$ & $\mathrm{ug} / \mathrm{L}$ \\
\hline$<10$ & u & & $<\mathrm{EQL}$ & $\mathrm{ug} / \mathrm{L}$ \\
\hline$<5$ & U & & $<\mathrm{EQL}$ & $\mathrm{ug} / \mathrm{L}$ \\
\hline$<10$ & $U$ & & $<E Q L$ & $\mathrm{ug} / \mathrm{L}$ \\
\hline$<8.28$ & JU & & $<\mathrm{EQL}$ & $\mathrm{ug} / \mathrm{L}$ \\
\hline & & & & $\mathrm{ug} / \mathrm{L}$ \\
\hline$<.095$ & U & & < EQL & ug/L \\
\hline$<5$ & $\mathrm{U}$ & & $<E Q L$ & $\mathrm{ug} / \mathrm{L}$ \\
\hline \multirow[t]{10}{*}{$<5$} & $U$ & & $<\mathrm{EQL}$ & $u g / L$ \\
\hline & & & & $\begin{array}{l}\text { ugg/L } \\
\mathrm{ug} / \mathrm{L}\end{array}$ \\
\hline & & & & ug/L \\
\hline & & & & ugh \\
\hline & & & & $\mathrm{ug} / \mathrm{L}$ \\
\hline & & & & $\mathrm{ug} / \mathrm{L}$ \\
\hline & & & & $\begin{array}{l}\mathrm{ug} / \mathrm{L} \\
\mathrm{ug} / \mathrm{L}\end{array}$ \\
\hline & & & & $\mathrm{ug} / \mathrm{L}$ \\
\hline & & & & ug/h \\
\hline & & & & $\mathrm{ug} / \mathrm{L}$ \\
\hline 5.02 & J & $Q$ & NDD & $\mathrm{pH}$ \\
\hline$<5$ & $U$ & & $<\mathrm{EQL}$ & $\mathrm{ug} / \mathrm{L}$ \\
\hline$<5$ & U & & $<$ EQL & $\mathrm{ug} / \mathrm{L}$ \\
\hline$<20$ & $U$ & & $<\mathrm{EQL}$ & $\mathrm{ug} / \mathrm{L}$ \\
\hline
\end{tabular}

Notes: Concentrations in bold italics exceed the groundwater protection standards listed in Appendix A. Bold italics were not assigned if the result was qualified with a "J", "R", "L", or "U" qualifier.

$+=$ exceeded the Groundwater Protection Standards listed in Appendix A. 
Table B. Assessment Monitoring Results for Groundwater Wells (Cont.)

WELL LFW 75D

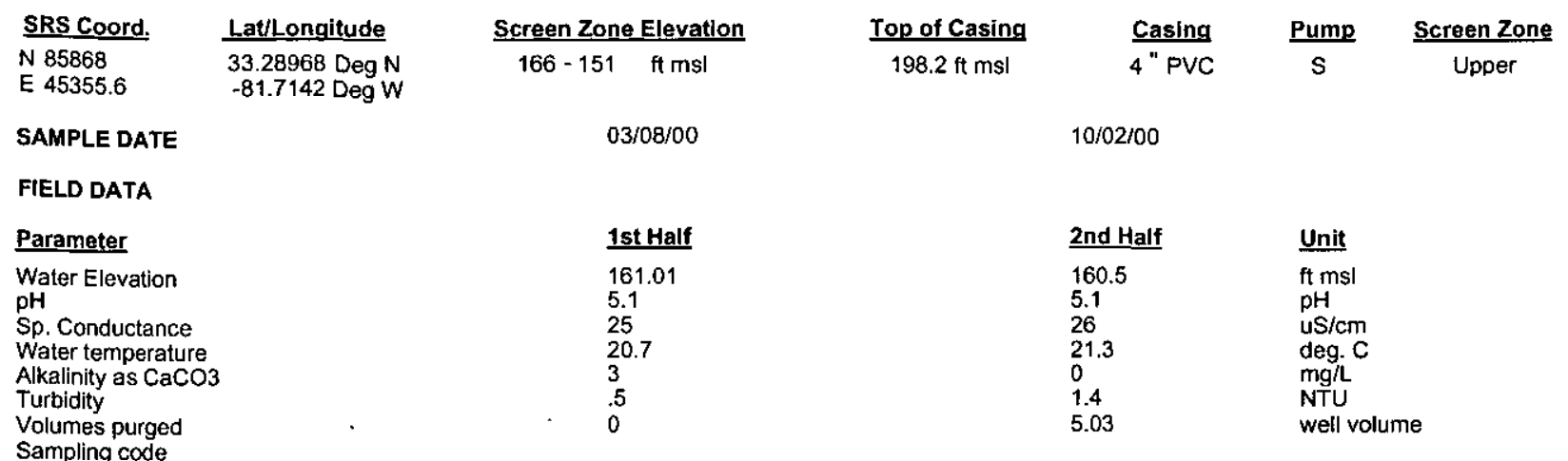

\section{ANALYTICAL DATA}

Groundwater Protection Standard

\section{ST Parameter}

1,1,1,2-Tetrachloroethane

1,1,1-Trichloroethane

1,1,2,2-Tetrachtoroethane

1,1,2-Trichloroethane

1,1-Dichloroethane

1,1-Dichloroethylene

1,1-Dichloropropene

1,2,3-Trichloropropane

$1,2,4,5$-Tetrachlorobenzene

1,2,4-Trichlorobenzene

1,2-Dibromo-3-chloropropane

1,2-Dibromoethane

1,2-Dichlorobenzene

1,2-Dichloroethane

1,2-Dichloropropane

1,3,5-Trinitrobenzene

1,3-Dichlorobenzene

1,3-Dichloropropane

1,3-Dinitrobenzene

1,4-Dichlorobenzene

1,4-Naphthoquinone

1-Naphthylamine

2,2-Dichloropropane

2,2-Oxybis(1-chloropropane)

$2,3,4,6$-Tetrachlorophenol

2,4,5-T

2,4,5-TP (Silvex)

2,4,5-Trichloropheno

2,4,6-Trichlorophenol

2,4-Dichlorophenol

2,4-Dichlorophenoxyacetic acid

2,4-Dimethyl phenol

2,4-Dinitrophenol

2,4-Dinitrotoluene

2,6-Dichlorophenol

2,6-Dinitrotoluene

2-Acetylaminofluorene

2-Chloronaphthalene

2-Chlorophenol

2-Hexanone

2-Methyl-4,6-dinitrophenol

2-Methylnaphthalene

2-Naphthylamine

2-Nitrophenol

2-sec-Butyl-4,6-dinitrophenol

3,3"-Dichlorobenzidine

3,3"-Dimethylbenzidine

3-Methylcholanthrene

4-Aminobiphenyl

4-Bromophenyl phenyl ether

4-Chloro-m-cresol

\begin{tabular}{|c|c|c|c|}
\hline Ist Halt & $\underline{\text { CLP }}$ & EPA & Fift \\
\hline$<5$ & U & & $<E$ \\
\hline$<5$ & $\mathrm{U}$ & & $<\mathrm{E}$ \\
\hline$<5$ & $\mathrm{U}$ & & \\
\hline$<5$ & $\mathrm{U}$ & & \\
\hline$<5$ & U & & $<\mathrm{E}$ \\
\hline$<5$ & $\mathrm{U}$ & & $<\mathrm{E}$ \\
\hline$<5$ & $\mathrm{U}$ & & \\
\hline$<5$ & $U$ & & \\
\hline$<53$ & JU & $Q$ & $<E$ \\
\hline$<11$ & JU & $Q$ & $<\mathrm{E}$ \\
\hline$<10$ & U & & $<E$ \\
\hline$<5$ & $\mathrm{U}$ & & $<\mathrm{E}$ \\
\hline$<5$ & $\mathrm{U}$ & & $<\mathrm{E}$ \\
\hline$<5$ & $U$ & & $<\mathrm{E}$ \\
\hline$<5$ & $\mathrm{U}$ & & $<E$ \\
\hline$<10$ & JU & $Q$ & $<\mathrm{E}$ \\
\hline$<5$ & U & & $<\mathrm{E}$ \\
\hline$<5$ & U & & $<\mathrm{E}$ \\
\hline$<50$ & $\mathrm{JU}$ & $Q$ & $<\mathrm{E}$ \\
\hline$<5$ & $U$ & & $<E$ \\
\hline$<11$ & ل & $Q$ & $<E C$ \\
\hline$<11$ & JU & $Q$ & $<E$ \\
\hline$<5$ & U & & $<E$ \\
\hline$<11$ & JU & $Q$ & $<E$ \\
\hline$<11$ & JU & $Q$ & $<E$ \\
\hline$<.2$ & $U$ & $Q$ & $<E$ \\
\hline$<.2$ & $\mathrm{U}$ & $Q$ & $<E$ \\
\hline$<11$ & JU & $Q$ & $<E$ \\
\hline$<26$ & JU & $Q$ & $<E$ \\
\hline$<11$ & JU & $Q$ & $<E$ \\
\hline$<.2$ & $\mathrm{U}$ & $Q$ & $<E$ \\
\hline$<11$ & JU & $Q$ & $<E$ \\
\hline$<26$ & JU & $Q$ & $<E$ \\
\hline$<11$ & JU & $Q$ & $<\mathrm{E}$ \\
\hline$<25$ & JU & $Q$ & $<E$ \\
\hline$<11$ & JU & $Q$ & $<\mathrm{E}$ \\
\hline$<11$ & JU & $\mathbf{Q}$ & $<E$ \\
\hline$<11$ & JU & $Q$ & $<E$ \\
\hline$<11$ & JU & $\mathrm{Q}$ & $<\mathrm{E}$ \\
\hline$<20$ & $\mathbf{U}$ & & $<\mathrm{E}$ \\
\hline$<26$ & JU & $Q$ & $<E$ \\
\hline$<11$ & JU & $Q$ & $<$ \\
\hline$<11$ & JU & $Q$ & $<E$ \\
\hline$<11$ & JU & $Q$ & \\
\hline$<.2$ & $U$ & $\mathbf{Q}$ & $<E$ \\
\hline$<11$ & JU & $\mathbf{Q}$ & $<\mathrm{E}$ \\
\hline$<21$ & JU & $\mathbf{Q}$ & $<\mathrm{E}$ \\
\hline$<11$ & JU & $Q$ & $<B$ \\
\hline$<11$ & $\mathrm{JU}$ & $Q$ & $<\mathrm{E}$ \\
\hline$<11$ & JU & $\mathrm{Q}$ & \\
\hline$<11$ & JU & $Q$ & \\
\hline
\end{tabular}

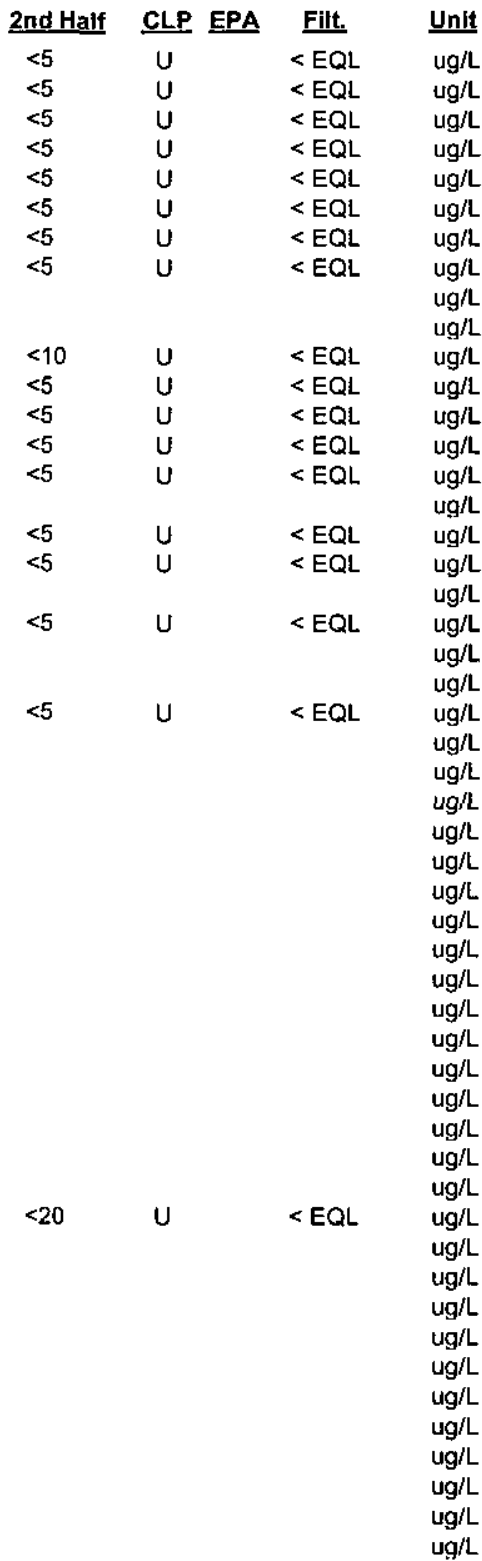

Notes: Concentrations in bold italics exceed the groundwater protection standards listed in Appendix A. Bold italics were not assigned if the result was qualified with a "J", "R", "L", or "U" qualifier.

$+=$ exceeded the Groundwater Protection Standards listed in Appendix A. 
Table B. Assessment Monitoring Results for Groundwater Wells (Cont.)

\section{WELL LFW 75D}

ANALYTICAL DATA

Groundwater Protection Standard

ST Parameter

4-Chloroaniline

4-Chlorophenyl phenyl ether

4-Nitrophenol

5-Nitro-o-toluidine

7,12-Dimethylbenz(a)anthracene

Acenaphthene

Acenaphthylene

Acetone

Acetonitrile (Methyl cyanide)

Acetophenone

Acrolein

Acrylonitrile

Aldrin

Allyl chloride

Anthracene

Antimony, total recoverable

Arsenic, total recoverable

Barium, total recoverable

Benzene

Benzo(a)anthracene

Benzo(a)pyrene

Benzo(b)fluoranthene

Benzo(g,h,i)perylene

Benzo(k)fluoranthene

Benzyl alcohol

Beryllium, total recoverable

Bis (2-chloroethoxy) methane

Bis(2-chloroethyl) ether

Bis(2-ethylhexyl) phthalate

Bromochloromethane

Bromodichloromethane

Bromoform

Bromomethane (Methyl bromide)

Butylbenzyl phthalate

Cadmium, total recoverable

Carbon disulfide

Carbon tetrachloride

Chlorobenzene

Chlorobenzilate

Chloroethane

Chloroethene (Vinyl chloride)

Chloroform

Chloromethane (Methyl chloride)

Chloroprene

Chromium, total recoverable

Chrysene

Cobalt total recoverable

Copper, total recoverable

Cyanide

Di-n-butyl phthalate

Di-n-octyl phthalate

Diallate

Dibenz(a,h)anthracene

Dibenzofuran

Dibromochloromethane

Dibromomethane (Methylene bromide)

Dichlorodifluoromethane

Dichloromethane (Methylene chloride)

Dieldrin

Diethyl phthalate

Dimethoate

Dimethyl phthalate

Diphenylamine

Disulfoton

Endosulfan I

Endosulfan II

Endosulfan sulfate

1st Half CLP EPA Filt.

$<11$ JU $Q<E Q L$

$<11$ JU $Q<E Q L$

$<26$ JU $Q<E Q L$

$<11$ JU $Q<E Q$

$<11$ JU $Q<E Q$

$<11$ JU $Q<E Q L$

$<11$ JU $Q<E Q$

$<20 \quad U<E Q$

$<200 \quad U<E Q$

$<11$ JU $Q<E Q L$

$<50 \quad U<$ EQL

$<10 \quad U<$ EQL

$<1 \quad U \quad<E Q$

$<5 \quad U \quad<E Q L$

$<11$ JU $Q<E Q L$

$<100 \quad U<$ EQL

$<10 \quad U<$ EQL

$\begin{array}{lll}11.6 & & <2000 \\ <5 & U & <E Q L\end{array}$

$<11$ JU $Q<E Q L$

$<11$ JU $Q \quad<E Q L$

$<11$ JU $Q<E Q L$

$<11$ JU $Q<E Q$

$<11$ JU $Q<E Q L$

$<1 \quad \mathrm{U} \quad<\mathrm{EQ}$

$<11$ JU $Q \quad<E Q L$

$<11$ JU $Q<E Q L$

$<11$ JU $Q<E Q L$

$<5 \quad U<$ EQL

$<5 \quad U<E Q L$

$<5 \quad U \quad<E Q L$

$<11$ JU $Q \quad<E Q L$

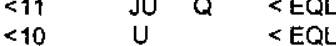

$<5 \quad U<E Q$

$<5 \quad U<E Q L$

$<5 \quad U \quad<E Q L$

$<11$ JU $Q<E Q L$

$<5 \quad U<E Q$

$<5 \quad U<E Q$

$<5 \quad U \quad<E Q$

$<5 \quad U \quad<E Q L$

$<20 \quad U<E Q$

$<10 \quad U<E Q$

$<11$ JU $Q<E Q L$

$<20 \quad U \quad<E Q$

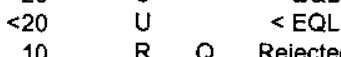

$<11$ JU $Q \quad<E Q$

$<11$ JU $Q \quad<E Q L$

$<11$ JU $Q<E Q L$

$<11$ JU $Q<E Q L$

$<11$ JU $Q<E Q L$

$<5 \quad U<E Q$

$<5 \quad U \quad<E Q L$

$<5 \quad U \quad<E Q$

$\begin{array}{llll}1.8 & J & \text { I } & \text { NDD }\end{array}$

$<.21 \quad U \quad<E Q L$

$<11$ JU $Q<E Q$

$<11$ JU $Q<E Q L$

$<11$ JU $Q<E Q L$

$<11$ JU $Q<E Q L$

$<11$ JU $Q<E Q L$

$<.1 \quad U<E Q$

$<.21 \quad U \quad<E Q$

$<.21 \quad U \quad<E Q L$

2nd Half CLP EPA Filt. Unit

\begin{tabular}{|c|c|c|}
\hline$<20$ & $U$ & $<E Q L$ \\
\hline$<200$ & $U$ & $<E Q L$ \\
\hline$<50$ & $U$ & . $<\mathrm{EQL}$ \\
\hline$<10$ & $U$ & $<\mathrm{EQL}$ \\
\hline$<5$ & $U$ & $<\mathrm{EQL}$ \\
\hline$<100$ & $U$ & $<E Q L$ \\
\hline$<10$ & $U$ & $<E Q L$ \\
\hline 11.9 & & $<2000$ \\
\hline$<5$ & $U$ & $<E Q L$ \\
\hline
\end{tabular}

$<.39$ JU $\quad$ EQL

$\begin{aligned} & \text { ug/L } \\ & \text { ug/L }\end{aligned}$

$<5 \quad U \quad<E Q L \quad \mathrm{ug} / \mathrm{L}$

$<5-10$

$<5$

$\begin{array}{llll}<5 & \mathrm{U} & <\mathrm{EQL} & \mathrm{ug} / \mathrm{L} \\ <5 & \mathrm{U} & <\mathrm{EQL} & \mathrm{ug} / \mathrm{L}\end{array}$

ug/L

$<10 \quad U \quad<E Q L \quad u g / L$

$<5 \quad U \quad<E Q L \quad u g / L$

$\begin{array}{llll}<5 & U & <E Q L & \mathrm{ug} / \mathrm{L} \\ <5 & \mathrm{U} & <\mathrm{EQL} & \mathrm{ug} / \mathrm{L}\end{array}$

$<5 \quad \mathrm{U}<\mathrm{EQL} \quad \mathrm{ug} / \mathrm{L}$

$<5 \quad U<\mathrm{EQL} \quad \mathrm{ug} / \mathrm{h}$

$<5 \quad \mathrm{U} \quad<E Q L \quad \mathrm{ug} / \mathrm{L}$

$<5 \quad U \quad<E Q L \quad$ ug/L

$<20 \quad U \quad<E Q L \quad u g /$

$<10 \quad U \quad<E Q L \quad u g / L$

$<$ Ugl $/ \mathrm{L}$

$<1.9-\quad$ JU

Ug/L

$<9.8 \quad U \quad<E Q L \quad u g / L$

ug/L

ug/L

$\mathrm{ug} / \mathrm{L}$

ug/L

$\begin{array}{llll}<5 & U & <E Q L & \mathrm{ug} / \mathrm{L} \\ <5 & \mathrm{U} & <\mathrm{EQL} & \mathrm{ug} / \mathrm{L}\end{array}$

$\begin{array}{llll}<5 & \mathrm{U} & <\mathrm{EQL} & \mathrm{ug} / \mathrm{L} \\ <5 & \mathrm{U} & <\mathrm{EQL} & \mathrm{ug} / \mathrm{L}\end{array}$

$<10 \quad \mathrm{E} \quad \mathrm{EQL} \quad \mathrm{ug} / \mathrm{L}$

$\mathrm{ug} / \mathrm{L}$

$\mathrm{ug} / \mathrm{L}$

ug/L

$\mathrm{ug} / \mathrm{L}$

ug/L

ug $/$ L

$\mathrm{ug} / \mathrm{L}$

$\mathrm{ug} / \mathrm{L}$

ug/L

Endrin

$<.21$

$<E Q L$

$u g / L$

Notes: Concentrations in bold italics exceed the groundwater protection standards listed in Appendix A. Bold italics were not assigned if the result was qualified with a "J", "R", "L", or "U" qualifier.

$+=$ exceeded the Groundwater Protection Standards listed in Appendix A. 
Table B. Assessment Monitoring Results for Groundwater Wells (Cont.)

WELL LFW 75D

\section{ANALYTICAL DATA}

Groundwater Protection Standard

\section{ST Parameter}

Endrin aldehyde

Ethyl methacrylate

Ethyl methanesulfonate

Ethylbenzene

Famphur

Fluoranthene

Fluorene

Heptachlor

Heptachlor epoxide

Hexachlorobenzene

Hexachlorobutadiene

Hexachlorocyclopentadiene

Hexachtoroethane

Hexachloropropene

Indeno(1,2,3-c,d)pyrene

lodomethane (Methyl iodide)

Isobutyl alcohol

Isodrin

Isophorone

Isosafrole

Kepone

Lead, total recoverable

Lindane

Mercury, total recoverable

Methacrylonitrile

Methapyritene

Methoxychlor

Methyl ethyl ketone

Methyl isobutyl ketone

Methyl methacrylate

Methyl methanesulfonate

N-Nitrosodi-n-butylamine

$\mathrm{N}$-Nitrosodiethylamine

$\mathrm{N}$-Nitrosodimethylamine

$\mathrm{N}$-Nitrosodiphenylamine

N-Nitrosodipropylamine

$\mathrm{N}$-Nitrosomethylethylamine

$\mathrm{N}$-Nitrosopiperidine

$\mathrm{N}$-Nitrosopyrrolidine

Naphthalene

Nickel, total recoverable

Nitrobenzene

O,O,O-Triethyl phosphorothioate

$P C B$

PCB 1016

PCB 1232

PCB 1242

PCB 1248

PCB 1254

PCB 1260

Parathion

Parathion methyl

Pentachlorobenzene

Pentachloronitrobenzene

Pentachlorophenol

Phenacetin

Phenanthrene

Phenol

Phorate

Pronamid

Propionitrile

Pyrene

Selenium, total recoverable

Silver, total recoverable

Specific conductance

Styrene

Sulfide

\begin{tabular}{|c|c|c|c|}
\hline 1 st Half & CLP & EPA & Filt. \\
\hline$<.21$ & U & & $<\mathrm{EQL}$ \\
\hline$<5$ & U & & $<\mathrm{EQL}$ \\
\hline$<11$ & JU & $Q$ & $<E Q L$ \\
\hline$<5$ & U & & $<E Q L$ \\
\hline$<200$ & $\mathrm{JU}$ & $Q$ & $<\mathrm{EQL}$ \\
\hline$<11$ & JU & $Q$ & $<E Q L$ \\
\hline$<11$ & JU & $Q$ & $<E Q L$ \\
\hline$<.1$ & u & & $<\mathrm{EQL}$ \\
\hline$<.1$ & $u$ & & $<E Q L$ \\
\hline$<11$ & JU & $Q$ & $<E Q L$ \\
\hline$<11$ & JU & $Q$ & $<E Q L$ \\
\hline$<11$ & JU & $Q$ & $<E Q L$ \\
\hline$<11$ & JU & $Q$ & $<E Q L$ \\
\hline$<53$ & JU & $Q$ & $<\mathrm{EQL}$ \\
\hline$<11$ & JU & $Q$ & $<E Q L$ \\
\hline$<5$ & U & & $<E Q L$ \\
\hline$<500$ & $u$ & & $<E Q L$ \\
\hline$<11$ & JU & $Q$ & $<E Q L$ \\
\hline$<11$ & JU & $\mathbf{Q}$ & $<\mathrm{EQL}$ \\
\hline$<11$ & JU & $Q$ & $<\mathrm{EQL}$ \\
\hline$<11$ & JU & $\mathbf{Q}$ & $<E Q L$ \\
\hline 3.74 & $\mathrm{~J}$ & 1 & NDD \\
\hline$<.1$ & U & & $<E Q L$ \\
\hline$<.5$ & U & & $<E Q L$ \\
\hline$<200$ & U & & $<E Q L$ \\
\hline$<11$ & JU & $Q$ & $<\mathrm{EQL}$ \\
\hline$<1$ & U & & $<E Q L$ \\
\hline$<20$ & 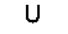 & & $<E Q L$ \\
\hline$<10$ & $u$ & & $<E Q L$ \\
\hline$<20$ & U & & $<E Q L$ \\
\hline$<11$ & JU & $Q$ & $<E Q L$ \\
\hline$<11$ & JU & $Q$ & $<\overrightarrow{E Q L}$ \\
\hline$<11$ & JU & $Q$ & $<\mathrm{EQL}$ \\
\hline$<26$ & JU & $Q$ & $<E Q L$ \\
\hline$<11$ & JU & $Q$ & $<E Q L$ \\
\hline$<11$ & JU & $Q$ & $<E Q L$ \\
\hline$<11$ & JU & $Q$ & $<E Q L$ \\
\hline$<11$ & JU & $Q$ & $<E Q L$ \\
\hline$<11$ & JU & $\mathrm{Q}$ & $<E Q L$ \\
\hline$<11$ & JU & $Q$ & $<\mathrm{EQL}$ \\
\hline$<50$ & $U$ & & $<\mathrm{EQL}$ \\
\hline$<11$ & JU & $Q$ & $<\mathrm{EQL}$ \\
\hline$<11$ & JU & $Q$ & $<\mathrm{EQL}$ \\
\hline$<1$ & $U$ & & $<E Q L$ \\
\hline$<1$ & $U$ & & $<\mathrm{EQL}$ \\
\hline$<2.1$ & u & & $<E Q L$ \\
\hline$<1$ & U & & $<\mathrm{EQL}$ \\
\hline$<1$ & U & & $<E Q L$ \\
\hline$<1$ & $u$ & & $<\mathrm{EQL}$ \\
\hline$<11$ & JU & $Q$ & $<E Q L$ \\
\hline$<11$ & JU & $Q$ & $<E Q L$ \\
\hline$<53$ & Ju & $Q$ & $<E Q L$ \\
\hline$<11$ & JU & $Q$ & $<E Q L$ \\
\hline$<26$ & JU & $Q$ & $<E Q L$ \\
\hline$<11$ & JU & $Q$ & $<E Q L$ \\
\hline$<11$ & JU & $Q$ & $<E Q L$ \\
\hline$<11$ & JU & $Q$ & $<E Q L$ \\
\hline$<11$ & JU & $Q$ & $<\mathrm{EQL}$ \\
\hline$<11$ & JU & $Q$ & $<E Q L$ \\
\hline$<200$ & $U$ & & $<E Q L$ \\
\hline$<11$ & JU & $\mathbf{Q}$ & $<E Q L$ \\
\hline$<11$ & JU & $\mathbf{Q}$ & $<E Q L$ \\
\hline$<10$ & U & & $<E Q L$ \\
\hline$<20$ & u & & $<\mathrm{EQL}$ \\
\hline 24 & & & $<37$ \\
\hline$<5$ & $u$ & & $<E Q L$ \\
\hline 1000 & $\mathbf{R}$ & Q & Rejected \\
\hline
\end{tabular}

Notes: Concentrations in bold italics exceed the groundwater protection standards listed in Appendix A. Bold italics were not assigned if the result was qualified with a "J", "R", "L", or "U" qualifier.

$+=$ exceeded the Groundwater Protection Standards listed in Appendix A. 
Table B. Assessment Monitoring Results for Groundwater Wells (Cont.)

WELL LFW 75D

ANALYTICAL DATA

Groundwater Protection Standard

ST Parameter

Tetrachloroethylene

Thallium, total recoverable

Thionazin

Tin, total recoverable

Toluene

Toxaphene

Trichloroethylene

Trichlorofluoromethane

Vanadium, total recoverable

Vinyl acetate

Xylenes

Zinc, total recoverable

aipha-Benzene hexachloride alpha-Chlordane

beta-Benzene hexachloride

cis-1,2-Dichloroethylene

cis-1,3-Dichloropropene

delta-Benzene hexachloride

gamma-Chlordane

m-Cresol (3-Methylphenol)

m-Nitroaniline

o-Cresol (2-Methylphenol)

o-Nitroaniline

o-Toluidine

p.p"-DDD

$p, p "-D D E$

p.p"-DDT

p-Cresol (4-Methylphenol)

p-Dimethylaminoazobenzene

p-Nitroaniline

p-Phenylenediamine

$\mathrm{pH}$

trans-1,2-Dichloroethylene

trans-1,3-Dichloropropene

trans-1,4-Dichloro-2-butene

\begin{tabular}{|c|c|c|c|}
\hline 1st Half & CLP & EPA & Filt. \\
\hline$<5$ & U & & $<E Q L$ \\
\hline$<10$ & U & & $<E Q L$ \\
\hline$<11$ & JU & $Q$ & $<E Q L$ \\
\hline$<200$ & U & & $<\mathrm{EQL}$ \\
\hline$<5$ & U & & $<E Q L$ \\
\hline$<2.1$ & U & & $<E Q L$ \\
\hline$<5$ & U & & $<E Q L$ \\
\hline 13 & & & $<20$ \\
\hline$<10$ & U & & $<E Q L$ \\
\hline$<5$ & U & & $<E Q L$ \\
\hline$<10$ & U & & $<E Q L$ \\
\hline 28 & & & $<29.3$ \\
\hline$<.1$ & U & & $<\mathrm{EQL}$ \\
\hline$<.1$ & u & & $<E Q L$ \\
\hline$<.1$ & U & & $<E Q L$ \\
\hline$<5$ & U & & $<E Q L$ \\
\hline$<5$ & U & & $<E Q L$ \\
\hline$<.1$ & U & & $<E Q L$ \\
\hline$<.1$ & U & & $<E Q L$ \\
\hline$<26$ & JU & $Q$ & $<E Q L$ \\
\hline$<11$ & JU & $Q$ & $<\mathrm{EQL}$ \\
\hline$<26$ & JU & $Q$ & $<E Q L$ \\
\hline$<11$ & JU & $Q$ & $<E Q L$ \\
\hline$<.21$ & U & & $<E Q L$ \\
\hline$<.21$ & $U$ & & $<E Q L$ \\
\hline$<.21$ & U & & $<E Q L$ \\
\hline$<10$ & JU & $Q$ & $<\mathrm{EQL}$ \\
\hline$<11$ & JU & $Q$ & $<\mathrm{EQL}$ \\
\hline$<11$ & JU & $Q$ & $<\mathrm{EQL}$ \\
\hline$<11$ & JU & $Q$ & $<\mathrm{EQL}$ \\
\hline 5.81 & $\mathbf{J}$ & $Q$ & NDD \\
\hline$<5$ & U & & $<\mathrm{EQL}$ \\
\hline$<5$ & U & & $<E Q L$ \\
\hline$<20$ & U & & $<\mathrm{EQL}$ \\
\hline
\end{tabular}

\begin{tabular}{|c|c|c|c|}
\hline 2nd Half & $\underline{\text { CLP }}$ & EPA & Filt. \\
\hline$<5$ & u & & $<E$ \\
\hline$<10$ & $U$ & & \\
\hline$<5$ & U & & \\
\hline$<5$ & $u$ & & $<\mathrm{E}$ \\
\hline 21 & $\mathbf{J}$ & $K$ & \\
\hline$<10$ & U & & \\
\hline$<5$ & U & & 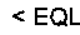 \\
\hline$<10$ & U & & 5 \\
\hline 21.5 & & & \\
\hline$<.095$ & $U$ & & \\
\hline$<5$ & $u$ & & \\
\hline$<5$ & U & & \\
\hline
\end{tabular}

Unit
ug/L
$u g / L$
$u g / L$
$u g / L$
$u g / L$
$u g / L$
$u g / L$
$u g / L$
$u g / L$
$u g / L$
$u g / L$
$u g / L$
$u g / L$
$u g / L$
$u g / L$
$u g / L$
$u g / L$
$u g / L$
$u g / L$
$u g / L$
$u g / L$
$u g / L$
$u g / L$
$u g / L$
$u g / L$
$u g / L$
$u g / L$
$u g / L$
$u g / L$
$u g / L$
$\mathrm{pH} /$
$u g / L$
$u g / L$
$u g / L$

Notes: Concentrations in bold italics exceed the groundwater protection standards listed in Appendix A. Bold italics were not assigned if the result was qualified with a "J", "R", "L", or "U" qualifier.

$+=$ exceeded the Groundwater Protection Standards listed in Appendix A. 
Table B. Assessment Monitoring Results for Groundwater Wells (Cont.)

WELL LFW 76

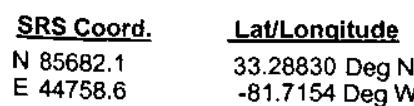

SAMPLE DATE

FIELD DATA

Parameter

Water Elevation

$\mathrm{pH}$

Sp. Conductance

Water temperature

Alkalinity as $\mathrm{CaCO} 3$

Turbidity

Volumes purged

Sampling code

ANALYTICAL DATA

Groundwater Protection Standard

ST Parameter

1,1,1,2-Tetrachloroethane

1,1,1-Trichloroethane

1,1,2,2-Tetrachioroethane

1,1,2-Trichloroethane

1,1-Dichloroethane

1,1-Dichloroethylene

1,1-Dichloropropene

1,2,3-Trichloropropane

1,2,4,5-Tetrachlorobenzene

1,2,4-Trichlorobenzene

1,2-Dibromo-3-chloropropane

1,2-Dibromoethane

1,2-Dichlorobenzene

1,2-Dichloroethane

1,2-Dichloropropane

1,3,5-Trinitrobenzene

1,3-Dichlorobenzene

1,3-Dichtoropropane

1,3-Dinitrobenzene

1,4-Dichlorobenzene

1,4-Naphthoquinone

1-Naphthylamine

2,2-Dichloropropane

2,2-Oxybis(1-chloropropane)

2,3,4,6-Tetrachlorophenol

2,4,5-T

2,4,5-TP (Silvex)

2,4,5-Trichlorophenol

2,4,6-Trichlorophenol

2,4-Dichlorophenol

2,4-Dichlorophenoxyacetic acid

2,4-Dimethyl phenol

2,4-Dinitrophenol

2,4-Dinitrotoluene

2,6-Dichlorophenol

2,6-Dinitrotoluene

2-Acetylaminofluorene

2-Chloronaphthalene

2-Chlorophenol

2-Hexanone

2-Methyl-4,6-dinitrophenol

2-Methylnaphthalene

2-Naphthylamine

2-Nitrophenol

2-sec-Butyl-4,6-disitrophenol

3,3"-Dichlorobenzidine

3,3"-Dimethylbenzidine

3-Methylcholanthrene

4-Aminobiphenyl

4-Bromophenyl phenyl ether

4-Chloro-m-cresol

\begin{tabular}{l} 
Screen Zone Elevat \\
\hline $157.9-142.9 \mathrm{ft} \mathrm{msl}$ \\
$02 / 28 / 00$ \\
\\
1 st Half \\
157.69 \\
5 \\
25 \\
22.7 \\
2 \\
14 \\
0 \\
$\mathrm{NX}$
\end{tabular}

1st Hal

$<5$
$<5$
$<5$
$<5$
$<5$
$<5$
$<5$
$<5$
$<52$

$<5$

$<5$

$<52$

$<11$

$<10$

$<5$

$<11$

$<5$

$<10$

$<11$

$<5$

$<50$

$<11$

$<11$

$<11$

$<5$

$<11$

$<11$

$<.2$

$<11$

$<26$

$<11$

$<.2$

$<11$

$<26$

$<11$

$<25$

$<11$

$<11$

$<11$

$<11$

$<20$

$<26$

$<11$

$<11$

$<11$

$<.2$

$<11$

$<21$

$<11$

$<11$

$<11$

$<11$

\section{Top of Casing \\ $221.9 \mathrm{ft} \mathrm{msl}$}

$\frac{\text { Casing }}{2 \text { "PVC }}$

Pump

Screen Zone

$10 / 02 / 00$

2nd Half
157.13
5.4
23
35
0
25.2
7.34
NX

Unit

ft msl

$\mathrm{pH}$

uS/cm

deg. C

$\mathrm{mg} / \mathrm{L}$

well volume

Notes: Concentrations in bold italics exceed the groundwater protection standards listed in Appendix A. Bold italics were not assigned if the result was qualified with a "J", "R", "L", or "U" qualifier.

$+=$ exceeded the Groundwater Protection Standards listed in Appendix A.

2nd Half CLP EPA Filt. Un

$<5 \quad U \quad<E Q L \quad u g / L$

$<5 \quad U \quad \mathrm{EQL} \quad \mathrm{ug} / \mathrm{L}$

$<5 \quad U<E Q L \quad u g / h$

$<5 \quad U<E Q L \quad u g / L$

$<5 \quad U<E Q \quad \mathrm{ug} / \mathrm{L}$

$<5 \quad U \quad<E Q L \quad u g / L$

$<5 \quad \mathrm{U}<\mathrm{EQL} \quad \mathrm{ug} / \mathrm{L}$

$\begin{array}{lllll}<E Q L & <5 & \mathrm{E} & <\mathrm{EQL} & \mathrm{ug} / \mathrm{L} \\ <\mathrm{EQL} & & & \mathrm{ug} / \mathrm{L}\end{array}$

$<E Q L \quad \mathrm{ug} / \mathrm{L}$

$<E Q L<10 \quad U \quad<E Q L \quad \mathrm{ug} / \mathrm{L}$

$<$ EQL $<5 \quad U \quad<E Q L \quad u g / L$

$<E Q L \quad<5 \quad U \quad<E Q L \quad u g / L$

$<E Q L \quad<5 \quad U \quad<E Q L \quad \mathrm{ug} / \mathrm{L}$

$<E Q L \quad<5 \quad U \quad<E Q L \quad u g / L$

$<$ EQL

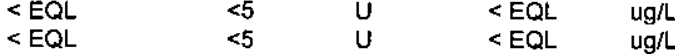

$<E Q L$

$<E Q L<5 \quad U \quad<E Q L \quad \mathrm{ug} / \mathrm{L}$

$<$ EQL $\quad u g / L$

$<$ EQL $\quad<$ ug/L

$<5<0$

$<E Q L \quad u g / L$

$<E Q L \quad \mathrm{ug} / \mathrm{L}$

$<E Q L \quad u g / h$

$<E Q L \quad \mathrm{ug} / \mathrm{L}$

$<E Q L \quad u g / L$

$<$ EQL $\quad$ ug/L

$<E Q L \quad u g / L$

ug/L

$<$ EQL $\quad u g / L$

$<$ EQL $\quad$ ug/L

$<$ EQL ug/L

$<$ EQL $\quad u g / L$

$<E Q L \quad u g / h$

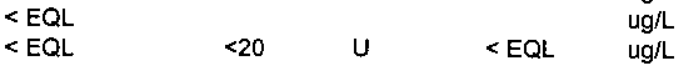

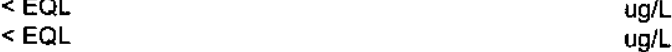

$<E Q L \quad u g / L$

$<E Q L \quad \mathrm{ug} / \mathrm{L}$

$<E Q L \quad u g / L$

$<$ EQL $\quad u g / h$

$<E Q L \quad u g / L$

$<$ EQL $\quad u g / L$

$<E Q L \quad \mathrm{ug} / \mathrm{L}$

$<E Q L \quad u g / L$

$\mathrm{ug} / \mathrm{L}$ 
Table B. Assessment Monitoring Results for Groundwater Wells (Cont.)

WELL LFW 76

ANALYTICAL DATA

Groundwater Protection Standard

ST Parameter

4-Chloroaniline

4-Chlorophenyl phenyl ether

4-Nitrophenol

5-Nitro-o-toluidine

7,12-Dimethylbenz(a)anthracene

Acenaphthene

Acenaphthylene

Acetone

Acetonitrile (Methyl cyanide)

Acetophenone

Acrolein

Acrylonitrile

Aldrin

Allyl chloride

Anthracene

Antimony, total recoverable

Arsenic, total recoverable

Barium, total recoverable

Benzene

Benzo(a)anthracene

Benzo(a)pyrene

Benzo(b)fluoranthene

Benzo(g,h,i)perylene

Benzo(k)fluoranthene

Benzyl alcohol

Beryllium, total recoverable

Bis(2-chloroethoxy) methane

Bis(2-chloroethyl) ether

Bis(2-ethylhexyl) phthalate

Bromochloromethane

Bromodichloromethane

Bromoform

Bromomethane (Methyl bromide)

Butylbenzyl phthatate

Cadmium, total recoverable

Carbon disulfide

Carbon tetrachloride

Chlorobenzene

Chlorobenzilate

Chloroethane

Chloroethene (Vinyl chloride)

Chloroform

Chloromethane (Methyl chloride)

Chloroprene

Chromium, total recoverable

Chrysene

Cobalt, total recoverable

Copper, total recoverable

Cyanide

Di-n-butyl phthalate

Di-n-octyl phthalate

Diallate

Dibenz(a,h)anthracene

Dibenzofuran

Dibromochloromethane

Dibromomethane (Methylene bromide)

Dichlorodifluoromethane

Dichloromethane (Methylene chloride)

Dieldrin

Diethyl phthalate

Dimethoate

Dimethyl phthalate

Diphenylamine

Disulfoton

Endosulfan I

Endosulfan II

Endosulfan sulfate

$\frac{1 \text { st Half }}{<11} \frac{\text { CLP EPA }}{U}$ Fllt.

$<11 \quad U \quad<E Q L$

$<26 \quad U \quad<E Q L$

$<11 \quad U<E Q$

$<11 \quad U \quad<E Q$

$<11 \quad U \quad<E Q L$

$<11 \quad U<E Q$

$<20 \quad U<E Q$

$<200 \quad U<E Q$

$<11 \quad U \quad<$ EQL

$<50 \quad U<E Q L$

$<10 \quad U<E Q L$

$<1 \quad U \quad<E Q L$

$<5 \quad U \quad<E Q L$

$<11 \quad U<E Q$

$<100 \quad U \quad<E Q L$

$<10 \quad U \quad<E Q L$

$12.7<2000$

$<5 \quad U \quad<$ EQL

$<11 \quad U \quad<E Q$

$<11 \quad U<E Q$

$<11 \quad U \quad<E Q L$

$<11 \quad \mathrm{E}<\mathrm{EQL}$

$<1 \quad U \quad<E Q$

$<11 \quad U \quad<E Q L$

$<11 \quad U \quad<E Q L$

$<11 \quad U \quad$ < EQL

$<5 \quad U \quad U E Q L$

$<5 \quad U<E Q$

$<5 \quad U<$ EQL

$<5 \quad U<E Q L$

$<11 \quad U \quad<E Q$

$<10 \quad U<E Q$

$<5 \quad U<E Q$

$<5 \quad U<E Q$

$<5 \quad U \quad<E Q L$

$<11 \quad U<E Q$

$<5 \quad U<E Q$

$<5 \quad U<E Q$

$<5 \quad U<E Q$

$<5 \quad U<E Q$

$<20 \quad U<E Q$

$<9.6 \quad U \quad V \quad<E Q L$

$<11 \quad U<E Q L$

$<6.21$ JU $V<E Q L$

$<7.16 \quad U \quad V \quad<E Q$

$<10 \quad U<E Q L$

$<11 \quad U$

$<11 \quad U<E Q$

$<11 \quad U<E Q$

$<11 \quad U \quad<E Q L$

$<11 \quad \mathrm{U}<E Q L$

$<5 \quad U<E Q$

$<5 \quad U<E Q$

$<5 \quad U<E Q L$

$<2.7 \quad U \quad V \quad<E Q$

$<.21 \quad U \quad<E Q L$

$<11 \quad U<E Q$

$<11 \quad U \quad<E Q$

$<11 \quad U \quad<E Q L$

$<11 \quad$ U

$<11 \quad U \quad<E Q$

$<1 \quad U \quad<E Q L$

$<21 \quad U \quad<E Q L$

$<.21 \quad U \quad<E Q L$

$<.21 \quad U \quad<E Q L$ 2nd Half CLP EPA Filt. Unit

$u g / L$

$\mathrm{ug} / \mathrm{L}$

ug/L

ug/L

ug/L

ug/L

ug/L

ug/L

$\mathrm{ug} / \mathrm{L}$

$\mathrm{ug} / \mathrm{L}$

$\mathrm{ug} / \mathrm{L}$

$\mathrm{ug} / \mathrm{L}$

$\mathrm{ug} / \mathrm{L}$

$\mathrm{ug} / \mathrm{L}$

$\mathrm{ug} / \mathrm{L}$

$\mathrm{ug} / \mathrm{L}$

$\mathrm{ug} / \mathrm{L}$

$\mathrm{ug} / \mathrm{L}$

$\mathrm{ug} / \mathrm{L}$

$\mathrm{ug} / \mathrm{L}$

$\mathrm{ug} / \mathrm{L}$

ug/L

ugh

ug/h

$<11 \quad$ JU $\quad$ EQL ug/

ug/l

ug/L

$\operatorname{ug} / \mathrm{L}$

$\begin{array}{llll}<5 & U & <E Q L & u g / L \\ <5 & U & <E Q L & u g / L\end{array}$

$\begin{array}{llll}<5 & \mathrm{U} & <\mathrm{EQL} & \mathrm{ug} / \mathrm{L} \\ <5 & \mathrm{U} & <\mathrm{EQL} & \mathrm{ug} / \mathrm{L}\end{array}$

$<10 \quad U \quad<E Q L \quad u g / L$

$<5 \quad U \quad<E Q L \quad u g / L$

$<5 \quad U$

$<E Q L$

ug/L

ug/L

ug/L

$<5 \quad U \quad<E Q L \quad u g / L$

$<5 \quad U<\mathrm{EQL} \quad \mathrm{ug} / \mathrm{L}$

$<5 \quad U \quad<E Q L \quad u g / L$

$<5 \quad U \quad<E Q L \quad u g / L$

$<20 \quad U \quad<E Q L \quad u g / h$

$25.8<100 \quad \mathrm{ug} / \mathrm{h}$

$<20 \quad U \quad<E Q L \quad u g /$

$<3.76 \mathrm{JU} \quad<$ EQL $\mathrm{ug} / \mathrm{L}$

$<\quad U<\mathrm{EQL} \quad \mathrm{ug} / \mathrm{L}$

ug/L

$\mathrm{ug} / \mathrm{l}$

$\mathrm{ug} / \mathrm{L}$

$\mathrm{ug} / \mathrm{L}$

$\begin{array}{llll}<5 & \mathrm{U} & <\mathrm{EQL} & \mathrm{ug} / \mathrm{L} \\ <5 & \mathrm{U} & <\mathrm{EQL} & \mathrm{ug} / \mathrm{L}\end{array}$

$\begin{array}{llll}<5 & \mathrm{U} & <\mathrm{EQL} & \mathrm{Ug} / \mathrm{L} \\ <5 & \mathrm{U} & <\mathrm{EQL} & \mathrm{ug} / \mathrm{L}\end{array}$

$<10 \quad U \quad<E Q L \quad u g / L$

$\mathrm{ug} / \mathrm{L}$

$u g / L$

$u g / L$

ug/L

ug/L

$\mathrm{ug} / \mathrm{L}$

$\mathrm{ug} / \mathrm{L}$

$u g / L$

$\mathrm{ug} / \mathrm{L}$

$\mathrm{ug} / \mathrm{L}$

Notes: Concentrations in bold italics exceed the groundwater protection standards tisted in Appendix A. Bold italics were not assigned if the result was qualified with a "J", "R", "L", or "U" qualifier.

$+=$ exceeded the Groundwater Protection Standards listed in Appendix A. 
Table B. Assessment Monitoring Results for Groundwater Wells (Cont.)

WELL LFW 76

ANALYTICAL DATA

Groundwater Protection Standard

ST Parameter

Endrin aldehyde

Ethyl methacrylate

Ethyl methanesulfonate

Ethylbenzene

Famphur

Fluoranthene

Fluorene

Heptachlor

Heptachlor epoxide

Hexachlorobenzene

Hexachlorobutadiene

Hexachlorocyclopentadiene

Hexachloroethane

Hexachloropropene

Indeno(1,2,3-c,d\}pyrene

lodomethane (Methyl iodide)

Isobutyl alcohol

Isodrin

Isophorone

Isosafrole

Kepone

Lead, total recoverable

Lindane

Mercury, total recoverable

Methacrylonitrile

Methapyrilene

Methoxychlor

Methyl ethyl ketone

Methyl isobutyl ketone

Methyl methacrylate

Methyl methanesulfonate

N-Nitrosodi-n-butylamine

$\mathrm{N}$-Nitrosodiethylamine

N-Nitrosodimethylamine

N-Nitrosodiphenylamine

N-Nitrosodipropylamine

N-Nitrosomethylethylamine

$\mathrm{N}$-Nitrosopiperidine

$\mathrm{N}$-Nitrosopyrrolidine

Naphthalene

Nickel, total recoverable

Nitrobenzene

O,O,O-Triethyl phosphorothioate

$\mathrm{PCB}$

PCB 1016

PCB 1232

PCB 1242

PCB 1248

PCB 1254

PCB 1260

Parathion

Parathion methy

Pentachlorobenzene

Pentachloronitrobenzene

Pentachlorophenol

Phenacetin

Phenanthrene

Phenol

Phorate

Pronamid

Propionitrile

Pyrene

Safrole

Selenium, total recoverable

Silver, total recoverable

Specific conductance

Styrene

Sulfide

\begin{tabular}{|c|c|c|c|}
\hline & CLL & EP & Filt. \\
\hline$<.21$ & U & & $<\mathrm{EQL}$ \\
\hline$<5$ & U & & $<E Q L$ \\
\hline$<11$ & $U$ & & $<\mathrm{EQL}$ \\
\hline$<5$ & $U$ & & $<E Q L$ \\
\hline$<200$ & $U$ & & $<E Q L$ \\
\hline$<11$ & U & & $<E Q L$ \\
\hline$<11$ & $U$ & & $<\mathrm{EQL}$ \\
\hline$<.1$ & U & & $<\mathrm{EQL}$ \\
\hline$<.1$ & $U$ & & $<\mathrm{EQL}$ \\
\hline$<11$ & $U$ & & $<\mathrm{EQL}$ \\
\hline$<11$ & $U$ & & $<\mathrm{EQL}$ \\
\hline$<11$ & $u$ & & $<E Q L$ \\
\hline$<11$ & $U$ & & $<\mathrm{EQL}$ \\
\hline$<52$ & $\mathrm{U}$ & & $<$ EQL \\
\hline$<11$ & $U$ & & $<E Q L$ \\
\hline$<5$ & $\mathrm{U}$ & & $<\mathrm{EQL}$ \\
\hline$<500$ & $U$ & & $<\mathrm{EQL}$ \\
\hline$<11$ & U & & $<E Q L$ \\
\hline$<11$ & U & & $<\mathrm{EQL}$ \\
\hline$<11$ & $U$ & & $<E Q L$ \\
\hline$<11$ & $U$ & & $<\mathrm{EQL}$ \\
\hline 3.51 & $\mathrm{~J}$ & I & NDD \\
\hline$<.1$ & $U$ & & $<E Q L$ \\
\hline$<.5$ & $U$ & & $<\mathrm{EQL}$ \\
\hline$<200$ & $U$ & & $<E Q L$ \\
\hline$<11$ & U & & $<E Q L$ \\
\hline$<1$ & $U$ & & $<\mathrm{EQL}$ \\
\hline$<20$ & $U$ & & $<\mathrm{EQL}$ \\
\hline$<10$ & U & & $<E Q L$ \\
\hline$<20$ & U & & $<E Q L$ \\
\hline$<11$ & u & & $<\mathrm{EQL}$ \\
\hline$<11$ & U & & $<\mathrm{EQL}$ \\
\hline$<11$ & U & & $<E Q L$ \\
\hline$<26$ & $U$ & & $<\mathrm{EQL}$ \\
\hline$<11$ & U & & $<E Q L$ \\
\hline$<11$ & U & & $<E Q L$ \\
\hline$<11$ & $U$ & & $<\mathrm{EQL}$ \\
\hline$<11$ & U & & $<\mathrm{EQL}$ \\
\hline$<11$ & $U$ & & $<\mathrm{EQL}$ \\
\hline$<11$ & $U$ & & $<E Q L$ \\
\hline$<10$ & JU & & $<\mathrm{EQL}$ \\
\hline$<11$ & $U$ & & $<$ EQL \\
\hline$<11$ & $\mathrm{U}$ & & $<E Q L$ \\
\hline$<1$ & U & & $<E Q L$ \\
\hline$<1$ & U & & $<E Q L$ \\
\hline$<2.1$ & U & & $<E Q L$ \\
\hline$<1$ & $U$ & & $<E Q L$ \\
\hline$<1$ & $U$ & & $<\mathrm{EQL}$ \\
\hline$<1$ & U & & $<\mathrm{EQL}$ \\
\hline$<11$ & $U$ & & $<\mathrm{EQL}$ \\
\hline$<11$ & U & & $<E Q L$ \\
\hline$<52$ & U & & $<\mathrm{EQL}$ \\
\hline$<11$ & $U$ & & $<E Q L$ \\
\hline$<26$ & U & & $<\mathrm{EQL}$ \\
\hline$<11$ & $U$ & & $<E Q L$ \\
\hline$<11$ & U & & $<\mathrm{EQL}$ \\
\hline$<11$ & U & & $<\mathrm{EQL}$ \\
\hline$<11$ & $U$ & & $<\mathrm{EQL}$ \\
\hline$<11$ & U & & $<E Q L$ \\
\hline$<200$ & $\mathrm{U}$ & & $<E Q L$ \\
\hline$<11$ & $U$ & & $<\mathrm{EQL}$ \\
\hline$<11$ & U & & $<E Q L$ \\
\hline$<10$ & U & & $<E Q L$ \\
\hline$<4.74$ & JU & & $<$ EQL \\
\hline 24.2 & & & $<37$ \\
\hline$<5$ & U & & $<\mathrm{EQ}$ \\
\hline$<1000$ & $U$ & & $<\mathrm{EQ}$ \\
\hline
\end{tabular}

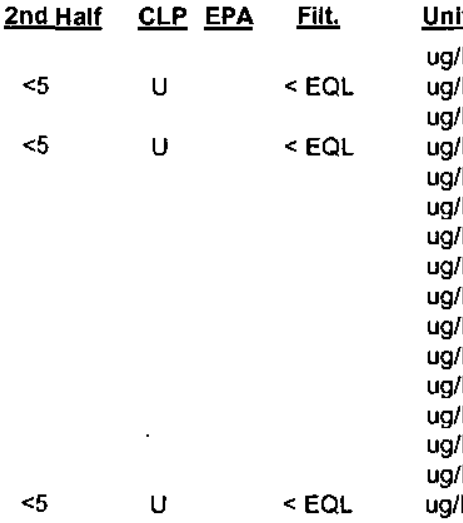

$<500 \quad U$

$\mathrm{ug} / \mathrm{L}$

$\mathrm{ug} / \mathrm{L}$

ug/L

$<10 \quad \mathrm{E} \quad \mathrm{EQL} \quad \mathrm{ug} / \mathrm{L}$

$<.5 \quad u$

$<200 \quad U<E Q L$

ug/L

ug/L

$<20 \quad U \quad<E Q L \quad u g / L$

$<10 \quad \mathrm{U} \quad<\mathrm{EQL} \quad \mathrm{ug} / \mathrm{L}$

$<20 \quad \mathrm{U} \quad<\mathrm{EQL} \quad \mathrm{ug} / \mathrm{L}$

$u g / L$

$\mathrm{ugh}$

$u g / L$

$\mathrm{ug} / \mathrm{L}$

$\mathrm{ug} / \mathrm{L}$

$\mathrm{ug} / \mathrm{L}$

$u g / L$

ug/L

$<10.9$ JU $\quad<$ EQL ug/L

$\mathrm{ug} / \mathrm{L}$

$\mathrm{ug} / \mathrm{L}$

ug/L

ug/L

ug $/ L$

$u g / L$

$u g / L$

ug $/ L$

$\mathrm{ug} / \mathrm{L}$

ug/L

$\mathrm{ug} / \mathrm{L}$

$u g / L$

ug/L

$\mathrm{ug} / \mathrm{L}$

ug/L

ug/L

$\mathrm{ug} / \mathrm{L}$

ug/

$\mathrm{ug} / \mathrm{L}$

ug/L

$\mathrm{ug} / \mathrm{L}$

ug/L

uS/cm

$\mathrm{ug} / \mathrm{L}$

Notes: Concentrations in bold italics exceed the groundwater protection standards listed in Appendix A. Bold italics were not assigned if the result was qualified with a "J", "R", "L", or "U" qualifier.

$+=$ exceeded the Groundwater Protection Standards listed in Appendix A. 
Table B. Assessment Monitoring Results for Groundwater Wells (Cont.)

WELL LFW 76

ANALYTICAL DATA

Groundwater Protection Standard

\section{ST Parameter}

Tetrachloroethylene

Thallium, total recoverable

Thionazin

Tin, total recoverable

Toluene

Toxaphene

Trichloroethylene

Trichlorofluoromethane

Vanadium, total recoverable

Vinyl acetate

Xylenes

Zinc, total recoverable

alpha-Benzene hexachloride alpha-Chlordane

beta-Benzene hexachloride

cis-1,2-Dichloroethylene

cis-1,3-Dichloropropene

delta-Benzene hexachloride

gamma-Chlordane

m-Cresol (3-Methyiphenol)

$\mathrm{m}$-Nitroaniline

o-Cresol (2-Methylphenol)

o-Nitroaniline

o-Toluidine

p.p"-DDD

$p, p "-D D E$

p.p"-DDT

p-Cresol (4-Methylphenol)

p-Dimethylaminoazobenzene

p-Nitroaniline

p-Phemylenediamine

$\mathrm{pH}$

trans-1,2-Dichloroethylene

trans-1,3-Dichloropropene

trans-1,4-Dichloro-2-butene

\begin{tabular}{|c|c|c|c|}
\hline 1st Half & $\underline{\text { CLP }}$ & EPA & Filt. \\
\hline$<5$ & $U$ & & $<$ EQL \\
\hline$<3.57$ & JU & & $<\mathrm{EQ}$ \\
\hline$<11$ & U & & $<E Q$ \\
\hline$<200$ & U & & $<E Q L$ \\
\hline$<5$ & U & & $<\mathrm{EQ}$ \\
\hline$<2.1$ & U & & $<E Q$ \\
\hline$<5$ & U & & $<E Q$ \\
\hline$<5$ & $U$ & & $<$ EQL \\
\hline$<2.36$ & JU & & $<\mathrm{EQ}$ \\
\hline$<5$ & $U$ & & $<E Q$ \\
\hline$<10$ & $U$ & & $<\mathrm{EQ}$ \\
\hline 35.6 & & & $>29$ \\
\hline$<.1$ & U & & $<\mathrm{EQ}$ \\
\hline$<.1$ & U & & $<$ EQL \\
\hline$<.1$ & U & & $<E Q$ \\
\hline$<5$ & $\mathrm{U}$ & & $<E Q$ \\
\hline$<5$ & $U$ & & $<E Q L$ \\
\hline$<.1$ & $U$ & & $<E Q$ \\
\hline$<.1$ & $U$ & & $<\mathrm{EQL}$ \\
\hline$<26$ & $U$ & & $<\mathrm{EQ}$ \\
\hline$<11$ & U & & $<\mathrm{EQL}$ \\
\hline$<26$ & U & & $<$ EQL \\
\hline$<11$ & $U$ & & $<\mathrm{EQL}$ \\
\hline$<.21$ & u & & $<\mathrm{EQ}$ \\
\hline$<.21$ & $u$ & & $<\mathrm{EQ}$ \\
\hline$<.21$ & $U$ & & $<\mathrm{EQ}$ \\
\hline$<10$ & $U$ & & $<\mathrm{EQ}$ \\
\hline$<11$ & $U$ & & $<E Q$ \\
\hline$<11$ & $\mathrm{U}$ & & $<E Q$ \\
\hline$<11$ & $U$ & & $<E Q$ \\
\hline 5.63 & $\mathrm{~J}$ & $Q$ & ND \\
\hline$<5$ & $\mathrm{U}$ & & $<\mathrm{EQ}$ \\
\hline$<5$ & U & & $<E Q$ \\
\hline$<20$ & $\mathrm{U}$ & & $<E Q$ \\
\hline
\end{tabular}



Notes: Concentrations in bold italics exceed the groundwater protection standards listed in Appendix A. Bold italics were not assigned if the result was qualified with a "J", "R", "L", or "U" qualifier.

$+=$ exceeded the Groundwater Protection Standards listed in Appendix A 
Table B. Assessment Monitoring Results for Groundwater Wells (Cont.)

WELL LFW 78

$\begin{array}{ll}\text { SRS Coord. } & \text { Lat/Longitude } \\ \text { N } 86064.9 & 33.28909 \text { Deg N } \\ \text { E 44726.5 } & -81.7162 \text { Deg W }\end{array}$

SAMPLE DATE

\section{FIELD DATA}

\section{Parameter}

Water Elevation

$\mathrm{pH}$

Sp. Conductance

Water temperature

Alkatinity as $\mathrm{CaCO} 3$

Turbidity

Volumes purged

Sampling code

\section{ANALYTICAL DATA}

Groundwater Protection Standard

\section{ST Parameter}

1,1,1,2-Tetrachloroethane

$1,1,1$-Trichloroethane

1,1,2,2-Tetrachloroethane

1,1,2-Trichloroethane

1,1-Dichloroethane

1,1-Dichloroethydene

1,1-Dichloropropene

1,2,3-Trichloropropane

1,2,4,5-Tetrachlorobenzene

1,2,4-Trichlorobenzene

1,2-Dibromo-3-chloropropane

1,2-Dibromoethane

1,2-Dichlorobenzene

1,2-Dichloroethane

1,2-Dichloropropane

1,3,5-Trinitrobenzene

1,3-Dichlorobenzene

1,3-Dichloropropane

1,3-Dinitrobenzene

1,4-Dichlorobenzene

1,4-Naphthoquinone

1-Naphthylarnine

2,2-Dichloropropane

2,2-Oxybis(1-chloropropane)

2,3,4,6-Tetrachlorophenol

$2,4,5-T$

2,4,5-TP (Silvex)

2,4,5-Trichlorophenol

2,4,6-Trichlorophenol

2,4-Dichtorophenol

2,4-Dichlorophenoxyacetic acid

2,4-Dimethyl phenol

2,4-Dinitrophenol

2,4-Dinitrotoluene

2,6-Dichlorophenol

2,6-Dinitrotoluene

2-Acetylaminofluorene

2-Chloronaphthalene

2-Chlorophenol

2-Hexanone

2-Methyl-4,6-dinitrophenol

2-Methylnaphthalene

2-Naphthylamine

2-Nitrophenol

2-sec-Butyl-4,6-dinitrophenol

3,3"-Dichlorobenzidine

3,3"-Dimethylbenzidine

3-Methylcholanthrene

4-Aminobiphenyl

4-Bromophenyl phenyl ether

4-Chloro-m-cresol
Screen Zone Elevation

$164.92-149.9 \mathrm{ft} \mathrm{msl}$

$03 / 30 / 00$

1st Half
159.62
5
38
26.7
5
9.8
6.39

1st Half

$<5$

$<5$

JU $\mathrm{L}<\mathrm{EQL}$

$<5$ JU L $<$ EQL

$<5 \quad$ JU $L \quad<$ L $\quad$ LQL

$<5$ JU L $<$ EQL

$<5$ JU L $<E Q L$

$<5 \quad J U L \quad<E Q L$

$<52$ JU $Q<E Q L$

$<10$ JU $Q \quad<E Q L$

$<10$ JU $L<E Q$

$<5$ JU $L<E Q L$

$<10 \quad J U \quad Q \quad<E Q L$

$<5 \quad J U \quad L \quad<E Q L$

$<5$ JU $\mathrm{L} \quad<$ EQL

$<10$ JU $Q<E Q L$

$<10$ JU $Q<E Q L$

$<5 \quad$ JU $L \quad<E Q L$

$<50$ JU $Q<E Q L$

$<10$ JU $Q<E Q L$

$<10$ JU $Q \quad<E Q L$

$<10$ JU $Q<$ EQL

$<5 \quad$ JU L $<$ EQL

$<10$ JU $Q<E Q L$

$<10$ JU $Q<E Q L$

$<.2$ JU $Q<E Q L$

$<.2$ JU $Q<E Q L$

$<10$ JU $Q<E Q L$

$<26$ JU $Q<E Q L$

$<10$ JU $Q<E Q L$

$<.2$ JU $Q<E Q L$

$<10$ JU $Q<E Q L$

$<26$ JU $Q<E Q L$

$<10$ JU $Q<E Q L$

$<25$ JU $Q<E Q L$

$<10$ JU $Q<E Q L$

$<10$ JU $Q<E Q L$

$<10<$ JU $\mathrm{Q}<\mathrm{EQL}$

$<10$ JU $Q<E Q L$

$<20$ JU $L<E Q L$

$<26$ JU $\mathrm{Q}<\mathrm{EQL}$

$<10$ JU $Q<E Q L$

$<10$ JU $Q<E Q L$

$<10$ JU $Q<E Q L$

$<.2$ JU $L Q<E Q L$

$<10$ JU $Q<E Q L$

$<21$ JU $Q<E Q L$

$<10$ JU $Q<E Q L$

$<10$ JU $Q<E Q L$

$<10$ JU $Q<E Q L$

$\frac{\text { Casing }}{2 \text { " PVC }} \quad \frac{\text { Pump }}{V} \quad \frac{\text { Screen Zone }}{\text { Upper }}$

$09 / 29 / 00$

\begin{tabular}{ll} 
2nd Half & Unit \\
\hline 158.66 & $\mathrm{ft} \mathrm{msl}$ \\
4.8 & $\mathrm{pH}$ \\
35 & uS/cm \\
23.7 & deg. C \\
0 & $\mathrm{mg} / \mathrm{L}$ \\
8.5 & NTU \\
4.26 & well volume
\end{tabular}

2ndHaif CLP EPA Filt. Uni

$<5 \quad U \quad<E Q \quad u g / L$

$<5 \quad U \quad<E Q \quad u g / L$

$<5 \quad U \quad<E Q \quad u g / L$

$<5 \quad U \quad<$ EQL ug/L

$<5 \quad U \quad<$ EQL ug/L

$<5 \quad U \quad<$ EQL ug/

$<5 \quad U \quad<E Q \quad$ ugh

$<5 \quad U \quad<E Q L \quad u g / h$

ug/L

$<10 \quad U \quad<$ EQL Ug/L

$<5 \quad U \quad<\quad<Q \quad u g / L$

$<5 \quad U \quad<E Q L \quad u g / L$

$<5 \quad \mathrm{U} \quad<\mathrm{EQL} \quad \mathrm{ug} / \mathrm{L}$

$<5 \quad U \quad<$ EQL ug/L

ug/L

$\begin{array}{llll}<5 & U & <E Q L & \mathrm{Ug} / \mathrm{L} \\ <5 & \mathrm{U} & <\mathrm{EQL} & \mathrm{ug} / \mathrm{L}\end{array}$

ug/L

$<5 \quad U \quad<E Q L \quad u g / L$

$\mathrm{ug} / \mathrm{L}$
$\mathrm{ug} / \mathrm{L}$

$<5 \quad U<$ EQL ug/L

$\mathrm{ug} / \mathrm{L}$

$\mathrm{ug} / \mathrm{L}$

ug/L

ug/L

$\mathrm{ug} / \mathrm{L}$

ug/L

$\mathrm{ug} / \mathrm{L}$

$\mathrm{ug} / \mathrm{L}$

ug/L

ug/L

ug/L

ug/L

ug/L

$\mathrm{ug} / \mathrm{L}$

$\mathrm{ug} / \mathrm{L}$

ug $/ L$

ugh

ug/L

ug/L

ug/L

$\mathrm{ug} / \mathrm{L}$

ug/L

$\mathrm{ug} / \mathrm{L}$

ug/L

ug/L

ug/L

ug/L

$\mathrm{ug} / \mathrm{L}$

Notes: Concentrations in bold italics exceed the groundwater protection standards listed in Appendix A. Bold italics were not assigned if the result was qualified with a "J", "R", "L", or "U" qualifier.

$+=$ exceeded the Groundwater Protection Standards listed in Appendix A. 
Table B. Assessment Monitoring Results for Groundwater Wells (Cont.) WELL LFW 78

\section{ANALYTICAL DATA}

Groundwater Protection Standard

\section{ST Parameter}

4-Chloroaniline

4-Chjorophenyl.phenyl ether

4-Nitrophenol

5-Nitro-o-toluidine

7,12-Dimethylbenz(a)anthracene

Acenaphthene

Acenaphthylene

Acetone

Acetonitrile (Methyl cyanide)

Acetophenone

Acrolein

Acrylonitrile

Aldrin

Allyl chloride

Anthracene

Antimony, total recoverable

Arsenic, total recoverable

Barium, total recoverable

Benzene

Benzo(a)anthracene

Benzo(a)pyrene

Benzo(b)fluoranthene

Benzo(g,h,i)perylene

Benzo(k)fluoranthene

Benzyl alcohol

Beryllium, total recoverable

Bis(2-chloroethoxy) methane

Bis(2-chloroethyl) ether

Bis(2-ethylhexy) phthalate

Bromochloromethane

Bromodichloromethane

Bromoform

Bromomethane (Methyl bromide)

Butylbenzyl phthalate

Cadmium, total recoverable

Carbon disulfide

Carbon tetrachloride

Chlorobenzene

Chlorobenzilate

Chloroethane

Chloroethene (Vinyl chloride)

Chloroform

Chloromethane (Methyl chloride)

Chloroprene

Chromium, total recoverable

Chrysene

Cobalt, total recoverable

Copper, total recoverable

Cyanide

Di-n-butyl phthalate

Di-n-octyl phthalate

Diallate

Dibenz $(a, h) a n t h r a c e n e$

Dibenzofuran

Dibromochloromethane

Dibromomethane (Methylene bromide)

Dichlorodifluoromethane

Dichloromethane (Methylene chloride)

Dieldrin

Diethyl phthalate

Dimethoate

Dimethyl phthalate

Diphenylamine

Disulfoton

Endosulfan I

Endosulfan II

Endosulfan sulfate

Endrin

\begin{tabular}{|c|c|c|c|}
\hline 1st Half & CLP & EPA & Filt. \\
\hline$<10$ & JU & $Q$ & $<E Q L$ \\
\hline$<10$ & JU & $Q$ & $<\mathrm{EQL}$ \\
\hline$<26$ & JU & $Q$ & $<E Q L$ \\
\hline$<10$ & JU & $\bar{Q}$ & $<E Q L$ \\
\hline$<10$ & JU & $Q$ & $<E Q L$ \\
\hline$<10$ & JU & $\vec{Q}$ & $<E Q L$ \\
\hline$<10$ & JU & $\bar{Q}$ & $<E Q L$ \\
\hline$<20$ & JU & L & $<\mathrm{EQL}$ \\
\hline$<200$ & JU & L & $<E Q L$ \\
\hline$<10$ & JU & $Q$ & $<\overline{E Q L}$ \\
\hline$<50$ & JU & $L$ & $<E Q L$ \\
\hline$<10$ & JU & $\mathrm{L}$ & $<\overline{E Q L}$ \\
\hline$<.11$ & JU & $Q$ & $<E Q L$ \\
\hline$<5$ & JU & $L$ & $<E Q L$ \\
\hline$<10$ & JU & $Q$ & $<E Q L$ \\
\hline$<100$ & U & & $<E Q L$ \\
\hline$<10$ & U & & $<E Q L$ \\
\hline 9.43 & $\mathrm{~J}$ & I & NDD \\
\hline$<5$ & JU & L & $<E Q L$ \\
\hline$<10$ & JU & $Q$ & $<\mathrm{EQL}$ \\
\hline$<10$ & JU & $Q$ & $<\mathrm{EQL}$ \\
\hline$<10$ & JU & $Q$ & $<E Q L$ \\
\hline$<10$ & JU & $Q$ & $<\mathrm{EQL}$ \\
\hline$<10$ & JU & $Q$ & $<E Q L$ \\
\hline$<1$ & U & & $<E Q L$ \\
\hline$<10$ & JU & $Q$ & $<E Q L$ \\
\hline$<10$ & JU & $Q$ & $<E Q L$ \\
\hline$<3.9$ & JU & IQV & $<E Q L$ \\
\hline$<5$ & JU & L & $<\mathrm{EQL}$ \\
\hline$<5$ & JU & L & $<\overline{E Q L}$ \\
\hline$<5$ & JU & L & $<E Q L$ \\
\hline$<5$ & JU & L & $<E Q L$ \\
\hline$<10$ & JU & $Q$ & $<E Q L$ \\
\hline$<10$ & $U$ & & $<\overline{E Q L}$ \\
\hline$<5$ & JU & L & $<E Q L$ \\
\hline$<5$ & JU & L & $<E Q L$ \\
\hline$<5$ & JU & L & $<\mathrm{EQL}$ \\
\hline$<10$ & JU & $Q$ & $<E Q L$ \\
\hline$<5$ & JU & L & $<E Q L$ \\
\hline$<5$ & JU & L & $<\overline{E Q L}$ \\
\hline$<5$ & JU & L & $<E Q L$ \\
\hline$<5$ & JU & L & $<E Q L$ \\
\hline$<20$ & JU & L & $<E Q L$ \\
\hline$<10$ & $u$ & & $<E Q L$ \\
\hline$<10$ & JU & $Q$ & $<E Q L$ \\
\hline$<20$ & u & & $<E Q L$ \\
\hline 9.42 & $J$ & I & NDD \\
\hline$<10$ & JU & $Q$ & $<E Q L$ \\
\hline$<10$ & JU & $Q$ & $<\mathrm{EQL}$ \\
\hline$<10$ & JU & $\bar{Q}$ & $<\overline{E Q L}$ \\
\hline$<10$ & JU & $Q$ & $<E Q L$ \\
\hline$<10$ & JU & $Q$ & $<E Q L$ \\
\hline$<10$ & $\mathrm{JU}$ & $Q$ & $<\mathrm{EQL}$ \\
\hline$<5$ & JU & L & $<$ EQL \\
\hline$<5$ & JU & L & $<\mathrm{EQL}$ \\
\hline$<5$ & JU & L & $<E Q L$ \\
\hline$<10$ & JU & L & $<E Q L$ \\
\hline$<.21$ & JU & $Q$ & $<\mathrm{EQL}$ \\
\hline$<10$ & JU & $Q$ & $<E Q L$ \\
\hline$<10$ & JU & $Q$ & $<E Q L$ \\
\hline$<10$ & JU & $Q$ & $<E Q L$ \\
\hline$<10$ & JU & $Q$ & $<\overline{E Q L}$ \\
\hline$<10$ & JU & $Q$ & $<E Q L$ \\
\hline$<.11$ & JU & $Q$ & $<E Q L$ \\
\hline$<.21$ & JU & $Q$ & $<\mathrm{EQL}$ \\
\hline$<.21$ & JU & $Q$ & $<E Q L$ \\
\hline$<.21$ & JU & $Q$ & $<E Q L$ \\
\hline
\end{tabular}

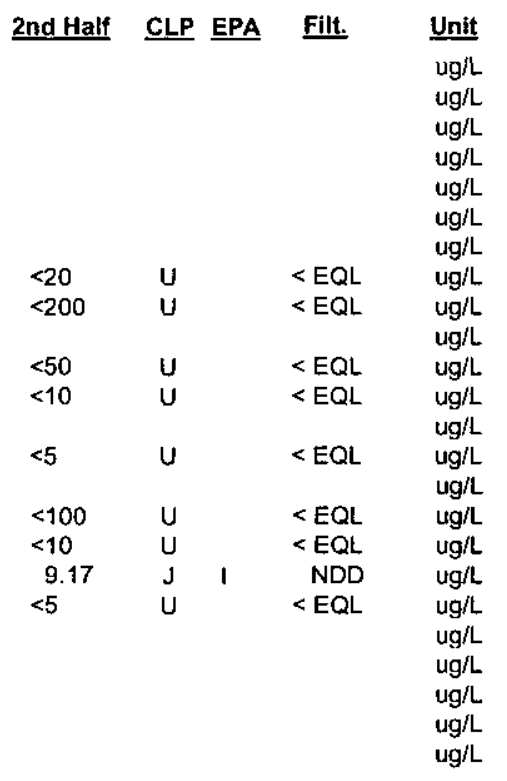

$<.12$ JU $<$ EQL Ug/L

$u g / L$

ug/ $/$

ugh

$\mathrm{ug} / \mathrm{L}$

$\begin{array}{llll}<5 & U & <E Q L & \mathrm{ug} / \mathrm{L} \\ <5 & U & <E Q L & \mathrm{ug} / \mathrm{L}\end{array}$

$<5 \quad U \quad<$ EQL Ug/L

$<5 \quad U \quad<E Q \quad$ ug/L

$<10 \quad U \quad<E Q L \quad \mathrm{ug} / \mathrm{L}$

$<5 \quad U \quad<E Q L \quad u g / L$

$<5 \quad U \quad<E Q L \quad u g / L$

$<5 \quad U \quad<E Q L \quad u g / L$

ugh $\mathrm{L}$

$\begin{array}{llll}<5 & U & <E Q L & u g / L \\ <5 & U & <E Q L & u g / L\end{array}$

$<5 \quad U<\mathrm{EQL}$

$<5 \quad U<\mathrm{EQL} \quad \mathrm{ug} / \mathrm{L}$

$<20 \quad U<$ EQL ug/L

$10.1<100 \quad$ ug/L

$<20 \quad \mathrm{U} \quad<\mathrm{EQL} \quad \mathrm{ug} / \mathrm{L}$

$24.5<29.8 \quad \mathrm{ug} / \mathrm{h}$

$<11$ JU $Q \quad<$ EQL ug/L

$<11 \quad$ JU $Q \quad<E Q L$ ug/L

$\mathrm{ug} / \mathrm{L}$

$u g / L$

ug/L

$<5 \quad U \quad<E Q L \quad$ ug/L

$<5 \quad U \quad<$ EQL $\mathrm{ug} / \mathrm{L}$

$<5 \quad U \quad<E Q L \quad \mathrm{ug} / \mathrm{h}$

$<10 \quad U<\mathrm{EQL} \quad \mathrm{ug} / \mathrm{L}$

$\mathrm{ug} / \mathrm{L}$

$\mathrm{ug} / \mathrm{L}$

$u g / L$

$u g / L$

ugh

ug/L

$\mathrm{ug} / \mathrm{L}$

$u g / L$

$\mathrm{ug} / \mathrm{L}$

$u g / L$

Notes: Concentrations in boid italics exceed the groundwater protection standards listed in Appendix $A$. Bold italics were not assigned if the result was qualified with a "J", "R", "L", or "U" qualifier.

$+=$ exceeded the Groundwater Protection Standards listed in Appendix A. 
Table B. Assessment Monitoring Results for Groundwater Wells (Cont.)

WELL LFW 78

ANALYTICAL DATA

Groundwater Protection Standard

ST Parameter

Endrin aldehyde

Ethyl methacrylate

Ethyl methanesulfonate

Ethylbenzene

Famphur

Fluoranthene

Fluorene

Heptachlor

Heptachlor epoxide

Hexachlorobenzene

Hexachlorobutadiene

Hexachlorocyclopentadiene

Hexachloroethane

Hexachloropropene

Indeno(1,2,3-c,d)pyrene

lodomethane (Methyl iodide)

Isobutyl alcohol

Isodrin

Isophorone

Isosafrole

Kepone

Lead, total recoverable

Lindane

Mercury, total recoverable

Methacrylonitrile

Methapyrilene

Methoxychlor

Methyl ethyl ketone

Methyl isobutyl ketone

Methyl methacrylate

Methyl methanesulfonate

$\mathrm{N}$-Nitrosodi-n-butylamine

$\mathrm{N}$-Nitrosodiethylamine

$\mathrm{N}$-Nitrosodimethylamine

$\mathrm{N}$-Nitrosodiphenylamine

$\mathrm{N}$-Nitrosodipropylamine

$\mathrm{N}$-Nitrosomethylethylamine

$\mathrm{N}$-Nitrosopiperidine

$\mathrm{N}$-Nitrosopyrrolidine

Naphthalene

Nickel, total recoverable

Nitrobenzene

O,0,0-Triethyl phosphorothioate

$\mathrm{PCB}$

PCB 1016

PCB 1232

PCB 1242

PCB 1248

PCB 1254

PCB 1260

Parathion

Parathion methyl

Pentachlorobenzene

Pentachloronitrobenzene

Pentachlorophenol

Phenacetin

Phenanthrene

Phenol

Phorate

Pronamid

Propionitrile

Pyrene

Safrole

Selenium, total recoverable

Silver, total recoverable

Specific conductance

Styrene

Sulfide

\begin{tabular}{|c|c|c|c|}
\hline 1st Half & CLㄹ & EPA & Filt. \\
\hline$<.21$ & JU & $Q$ & $<$ EQL \\
\hline$<5$ & JU & L & $<E Q L$ \\
\hline$<10$ & JU & $Q$ & $<E Q L$ \\
\hline$<5$ & JU & $\mathrm{L}$ & $<\mathrm{EQL}$ \\
\hline$<200$ & JU & $Q$ & $<$ EQL \\
\hline$<10$ & JU & $Q$ & $<E Q L$ \\
\hline$<10$ & JU & $Q$ & $<\mathrm{EQL}$ \\
\hline$<.11$ & JU & $Q$ & $<E Q L$ \\
\hline$<.11$ & JU & $a$ & $<E Q L$ \\
\hline$<10$ & JU & $Q$ & $<E Q L$ \\
\hline$<10$ & JU & $Q$ & $<E Q L$ \\
\hline$<10$ & JU & $\mathbf{Q}$ & $<$ EQL \\
\hline$<10$ & JU & $\mathbf{Q}$ & $<E Q L$ \\
\hline$<52$ & JU & $Q$ & $<$ EQL \\
\hline$<10$ & JU & $Q$ & $<\mathrm{EQL}$ \\
\hline$<5$ & $\mathrm{JU}$ & $L$ & $<\mathrm{EQ1}$ \\
\hline$<500$ & JU & L & $<E Q L$ \\
\hline$<10$ & JU & $Q$ & $<$ EQL \\
\hline$<10$ & JU & $Q$ & $<E Q L$ \\
\hline$<10$ & JU & $Q$ & $<\mathrm{EQL}$ \\
\hline$<10$ & JU & $Q$ & $<E Q L$ \\
\hline$<10$ & U & & $<E Q L$ \\
\hline$<.11$ & JU & $Q$ & $<\mathrm{EQL}$ \\
\hline$<.5$ & $U$ & & $<\mathrm{EQL}$ \\
\hline$<200$ & $J U$ & $\mathbf{L}$ & $<E Q L$ \\
\hline$<10$ & JU & $Q$ & $<\mathrm{EQL}$ \\
\hline$<1.1$ & JU & $\mathbf{Q}$ & $<\mathrm{EQL}$ \\
\hline$<20$ & JU & $L$ & $<E Q L$ \\
\hline$<10$ & JU & $\mathrm{L}$ & $<E Q L$ \\
\hline$<20$ & JU & $\mathrm{L}$ & $<\mathrm{EQL}$ \\
\hline$<10$ & $\mathrm{JU}$ & $Q$ & $<E Q L$ \\
\hline$<10$ & JU & $\mathbf{Q}$ & $<E Q L$ \\
\hline$<10$ & JU & $\mathbf{Q}$ & $<\mathrm{EQL}$ \\
\hline$<26$ & JU & $Q$ & $<E Q L$ \\
\hline$<10$ & JU & $Q$ & $<\mathrm{EQL}$ \\
\hline$<10$ & JU & $Q$ & $<\mathrm{EQL}$ \\
\hline$<10$ & JU & $Q$ & $<$ EQL \\
\hline$<10$ & JU & Q & $<$ EQL \\
\hline$<10$ & $\mathrm{JU}$ & $Q$ & $<$ EQL \\
\hline$<10$ & J & $Q$ & $<E Q L$ \\
\hline$<12.7$ & JU & 1 & $<E Q L$ \\
\hline$<10$ & JU & $Q$ & $<E Q L$ \\
\hline$<10$ & JU & $\mathbf{Q}$ & $<\mathrm{EQL}$ \\
\hline$<1.1$ & JU & $Q$ & < EQL \\
\hline$<1.1$ & JU & $Q$ & $<E Q L$ \\
\hline$<2.1$ & JU & $\mathbf{Q}$ & $<E Q L$ \\
\hline$<1.1$ & JU & $\mathbf{Q}$ & $<E Q L$ \\
\hline$<1.1$ & JU & $Q$ & $<E Q L$ \\
\hline$<1.1$ & JU & $\mathbf{Q}$ & $<E Q L$ \\
\hline$<10$ & JU & $Q$ & $<$ EQL \\
\hline$<10$ & JU & $Q$ & $<E Q L$ \\
\hline$<52$ & JU & $Q$ & $<E Q L$ \\
\hline$<10$ & JU & $Q$ & $<\mathrm{EQL}$ \\
\hline$<26$ & $\mathrm{JU}$ & $Q$ & $<E Q L$ \\
\hline$<10$ & JU & $\mathbf{Q}$ & $<E Q L$ \\
\hline$<10$ & JU & $\mathbf{Q}$ & $<\mathrm{EQL}$ \\
\hline$<10$ & JU & $Q$ & $<E Q L$ \\
\hline$<10$ & $\mathrm{JU}$ & $Q$ & $<\mathrm{EQL}$ \\
\hline$<10$ & JU & $Q$ & $<E Q L$ \\
\hline$<200$ & JU & $L$ & $<E Q L$ \\
\hline$<10$ & JU & $\mathbf{Q}$ & $<E Q L$ \\
\hline$<10$ & JU & $\mathbf{Q}$ & $<\mathrm{EQL}$ \\
\hline$<10$ & U & & $<\mathrm{EQL}$ \\
\hline$<20$ & U & & $<E Q L$ \\
\hline 39 & & & $>37$ \\
\hline$<5$ & لU & $L$ & $<E Q L$ \\
\hline$<1000$ & $\mathrm{U}$ & & $<E Q L$ \\
\hline
\end{tabular}

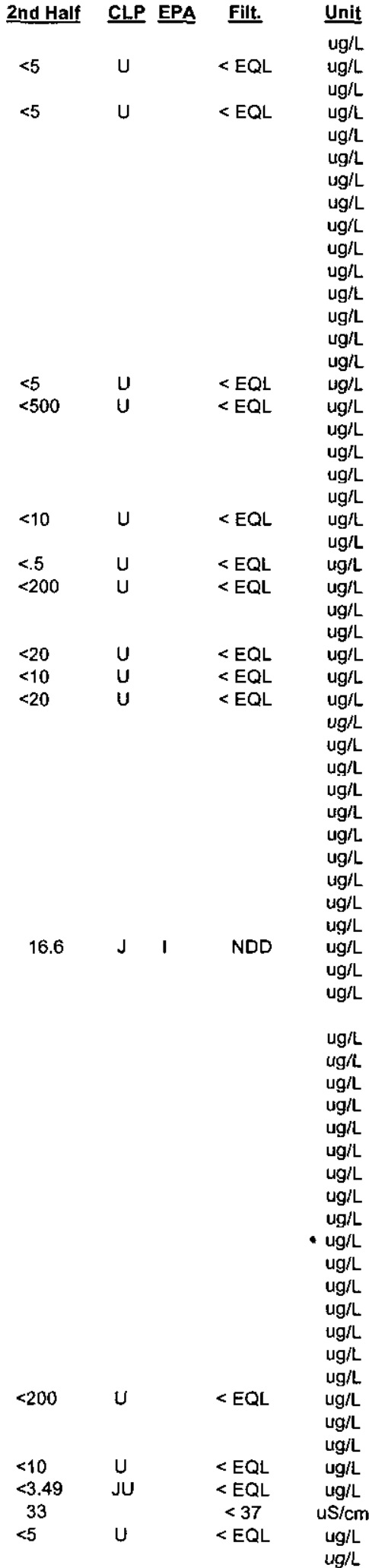

Notes: Concentrations in bold italics exceed the groundwater protection standards listed in Appendix A. Bold Italics were not assigned if the result was qualified with a "J", "R", "L", or "U" qualifier.

$+=$ exceeded the Groundwater Protection Standards listed In Appendix A. 
Table B. Assessment Monitoring Results for Groundwater Wells (Cont.)

WELL LFW 78

ANALYTICAL DATA

Groundwater Protection Standard

\section{ST Parameter}

Tetrachloroethylene

Thallium, total recoverable

Thionazin

Tin, total recoverable

Toluene

Toxaphene

Trichloroethylene

Trichlorofluoromethane

Vanadium, total recoverable

Vinyl acetate

Xylenes

$+\quad$ Zinc, total recoverable

alpha-Benzene hexachioride alpha-Chlordane

beta-Benzene hexachloride

cis-1,2-Dichloroethylene

cis-1,3-Dichloropropene

delta-Benzene hexachloride

gamma-Chlordane

m-Cresol (3-Methylphenol)

m-Nitroaniline

o-Cresol (2-Methylphenol)

o-Nitroaniline

o-Toluidine

$p, p=-D D D$

$p, p^{\prime \prime}-D D E$

p,p"-DDT

p-Cresol (4-Methylphenol)

p-Dimethylaminoazobenzene

p-Nitroaniline

p-Phenylenediamine

pH

trans-1,2-Dichloroethylene

trans-1,3-Dichloropropene

trans-1,4-Dichloro-2-butene

\begin{tabular}{|c|c|c|c|}
\hline 1st Half & CLP & EPA & Filt. \\
\hline$<5$ & JU & L & $<\mathrm{EQL}$ \\
\hline$<10$ & $U$ & & $<E Q L$ \\
\hline$<10$ & JU & $Q$ & $<E Q L$ \\
\hline$<200$ & U & & $<E Q L$ \\
\hline$<5$ & JU & L & $<E Q L$ \\
\hline$<2.1$ & JU & $Q$ & $<E Q L$ \\
\hline$<5$ & JU & L & $<\mathrm{EQL}$ \\
\hline 27 & $J$ & $\mathrm{~L}$ & NDD \\
\hline$<10$ & U & & $<\mathrm{EQL}$ \\
\hline$<5$ & JU & L & $<\mathrm{EQL}$ \\
\hline$<10$ & JU & L & $<E Q L$ \\
\hline 32.7 & & & $>29.3$ \\
\hline$<.11$ & UU & Q & $<E Q L$ \\
\hline$<.11$ & JU & $Q$ & $<E Q L$ \\
\hline$<.11$ & JU & $Q$ & $<E Q L$ \\
\hline$<5$ & JU & L & $<E Q L$ \\
\hline$<5$ & JU & L & $<\mathrm{EQL}$ \\
\hline$<.11$ & JU & $Q$ & $<E Q L$ \\
\hline$<.11$ & JU & $Q$ & $<\mathrm{EQL}$ \\
\hline$<26$ & U & $Q$ & $<\mathrm{EQL}$ \\
\hline$<10$ & JU & $Q$ & $<\mathrm{EQL}$ \\
\hline$<26$ & JU & $Q$ & $<E Q L$ \\
\hline$<10$ & JU & Q & $<E Q L$ \\
\hline$<.21$ & JU & $Q$ & $<E Q L$ \\
\hline$<.21$ & JU & $Q$ & $<E Q L$ \\
\hline$<.21$ & JU & $Q$ & $<\mathrm{EQL}$ \\
\hline$<10$ & JU & $Q$ & $<E Q L$ \\
\hline$<10$ & JU & $Q$ & $<E Q L$ \\
\hline$<10$ & JU & $Q$ & $<E Q L$ \\
\hline$<10$ & JU & $Q$ & $<E Q L$ \\
\hline 5.3 & $\mathrm{~J}$ & $Q$ & NDD \\
\hline$<5$ & JU & L & $<\mathrm{EQL}$ \\
\hline$<5$ & JU & $L$ & $<E Q L$ \\
\hline$<20$ & JU & & $<\mathrm{EQL}$ \\
\hline
\end{tabular}

\begin{tabular}{|c|c|c|c|}
\hline 2nd Half & CLPP & EPA & Filt \\
\hline$<5$ & U & & $<\mathrm{E}$ \\
\hline$<10$ & U & & \\
\hline$<5$ & u & & \\
\hline$<5$ & $U$ & & \\
\hline 25 & $\mathrm{~J}$ & K & \\
\hline$<10$ & U & & $\mathbf{E}$ \\
\hline$<5$ & U & & $<\theta$ \\
\hline$<10$ & $U$ & & \\
\hline 29.4 & & & \\
\hline$<.1$ & $u$ & & \\
\hline$<5$ & $u$ & & \\
\hline$<5$ & U & & \\
\hline
\end{tabular}

Unit
$\mathrm{ug} / \mathrm{L}$
$\mathrm{ug} / \mathrm{L}$
$\mathrm{ug} / \mathrm{L}$
$\mathrm{ug} / \mathrm{L}$
$\mathrm{ug} / \mathrm{L}$
$\mathrm{ug} / \mathrm{L}$
$\mathrm{ug} / \mathrm{L}$
$\mathrm{ug} / \mathrm{L}$
$\mathrm{ug} / \mathrm{L}$
$\mathrm{ug} / \mathrm{L}$
$\mathrm{ug} / \mathrm{L}$
$\mathrm{ug} / \mathrm{L}$
$\mathrm{ug} / \mathrm{L}$
$\mathrm{ug} / \mathrm{L}$
$\mathrm{ug} / \mathrm{L}$
$\mathrm{ug} / \mathrm{L}$
$\mathrm{ug} / \mathrm{L}$
$\mathrm{ug} / \mathrm{L}$
$\mathrm{ug} / \mathrm{L}$
$\mathrm{ug} / \mathrm{L}$
$\mathrm{ug} / \mathrm{L}$
$\mathrm{ug} / \mathrm{L}$
$\mathrm{ug} / \mathrm{L}$
$\mathrm{ug} / \mathrm{L}$
$\mathrm{ug} / \mathrm{L}$
$\mathrm{ug} / \mathrm{L}$
$\mathrm{ug} / \mathrm{L}$
$\mathrm{ug} / \mathrm{L}$
$\mathrm{ug} / \mathrm{L}$
$\mathrm{ug} / \mathrm{L}$
$\mathrm{pH}$
$\mathrm{ug} / \mathrm{L}$
$\mathrm{ug} / \mathrm{L}$
$\mathrm{ug} / \mathrm{L}$

Notes: Concentrations in bold italics exceed the groundwater protection standards listed in Appendix A. Bold italics were not assigned if the result was qualified with a "J", "R", "L", or "U" qualifier.

$+=$ exceeded the Groundwater Protection Standards listed in Appendix A. 


\section{Appendix C}

\section{Figures}


THIS PAGE LEFT BLANK INTENTIONALLY 




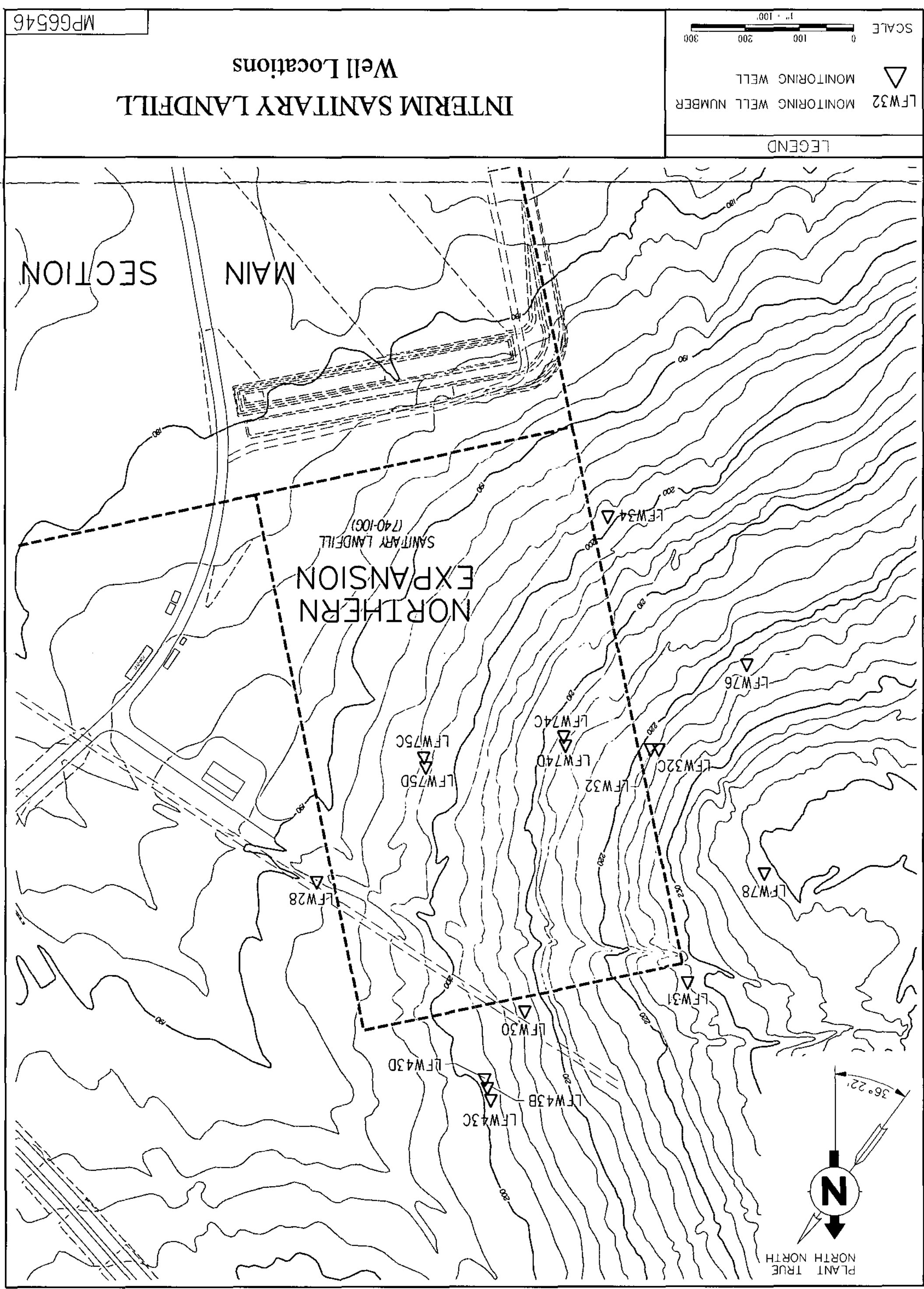

I00Z XYVANVI: ว1e $\mathrm{a}$ 


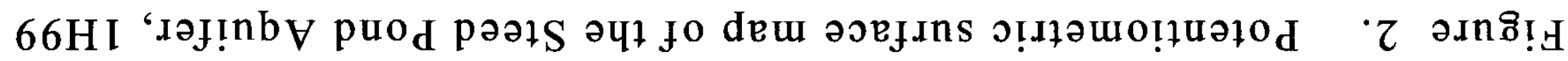

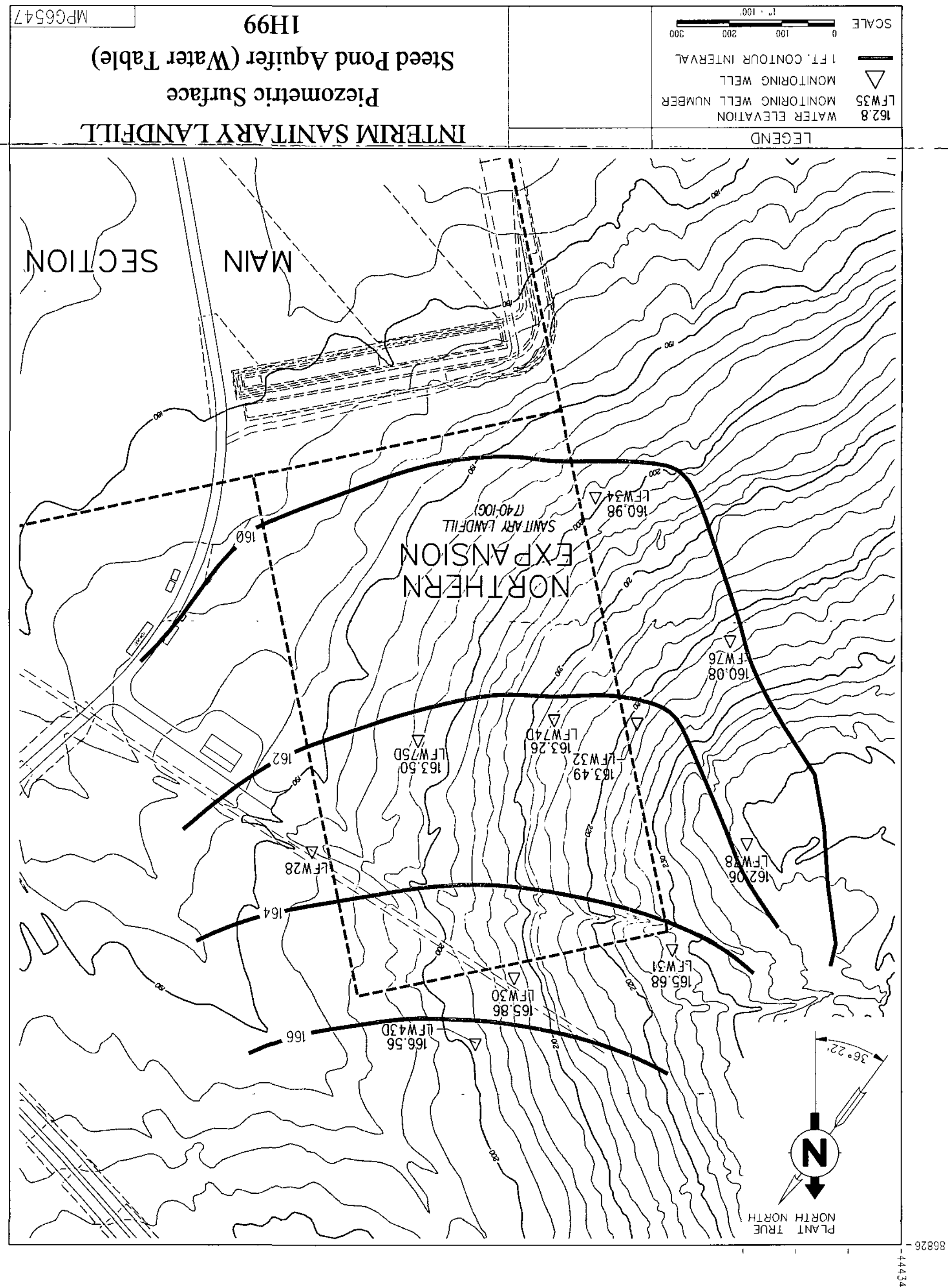




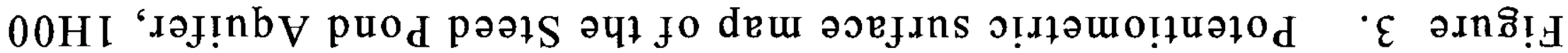

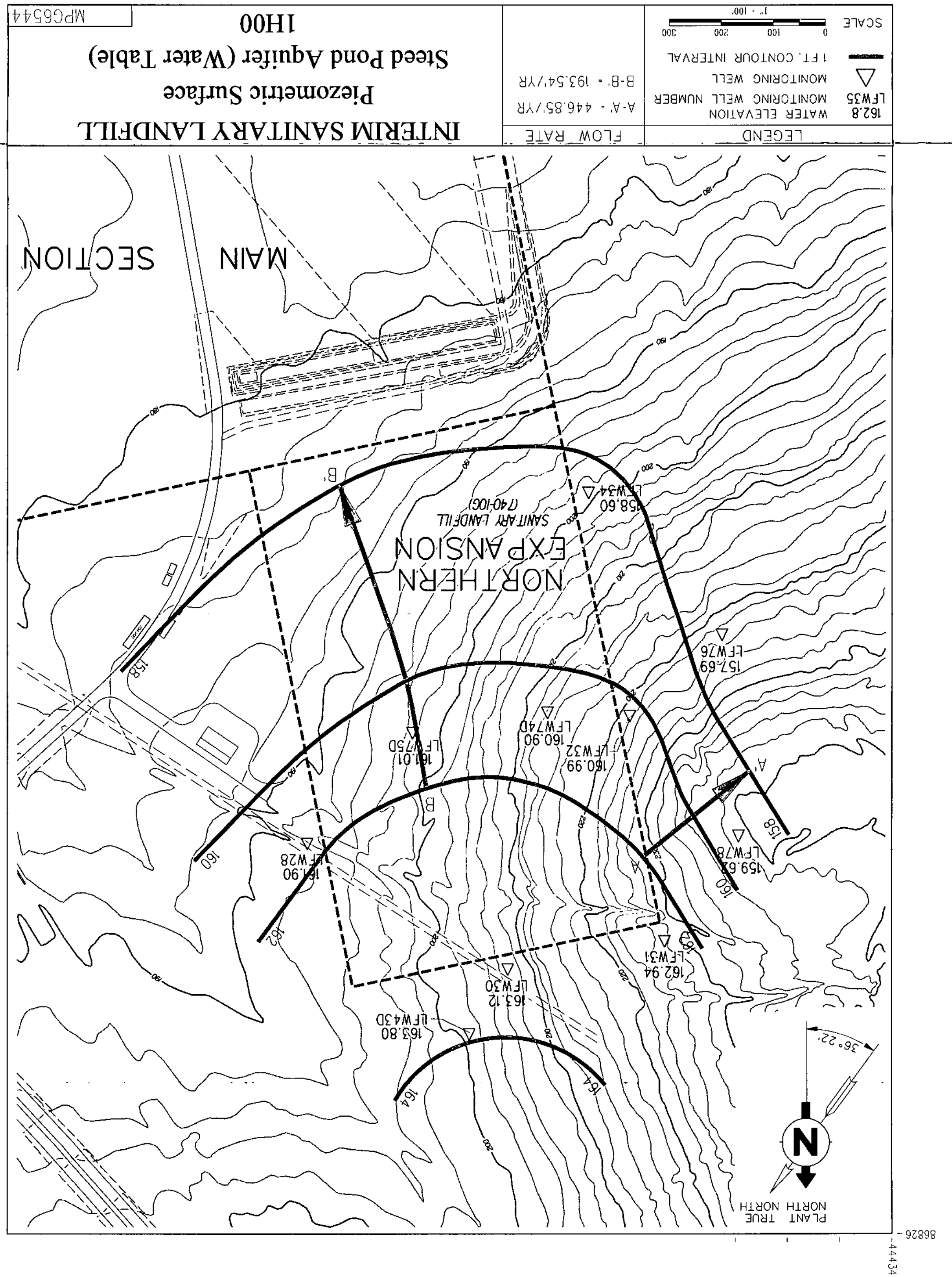



$\downarrow 9+00-000 z-\mathrm{U} L-\supset \mathrm{dSM}$ 


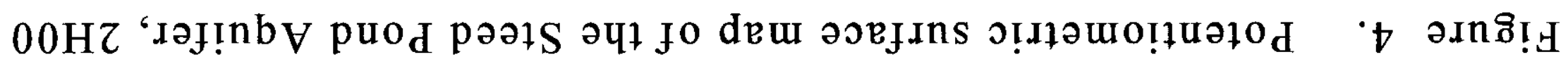

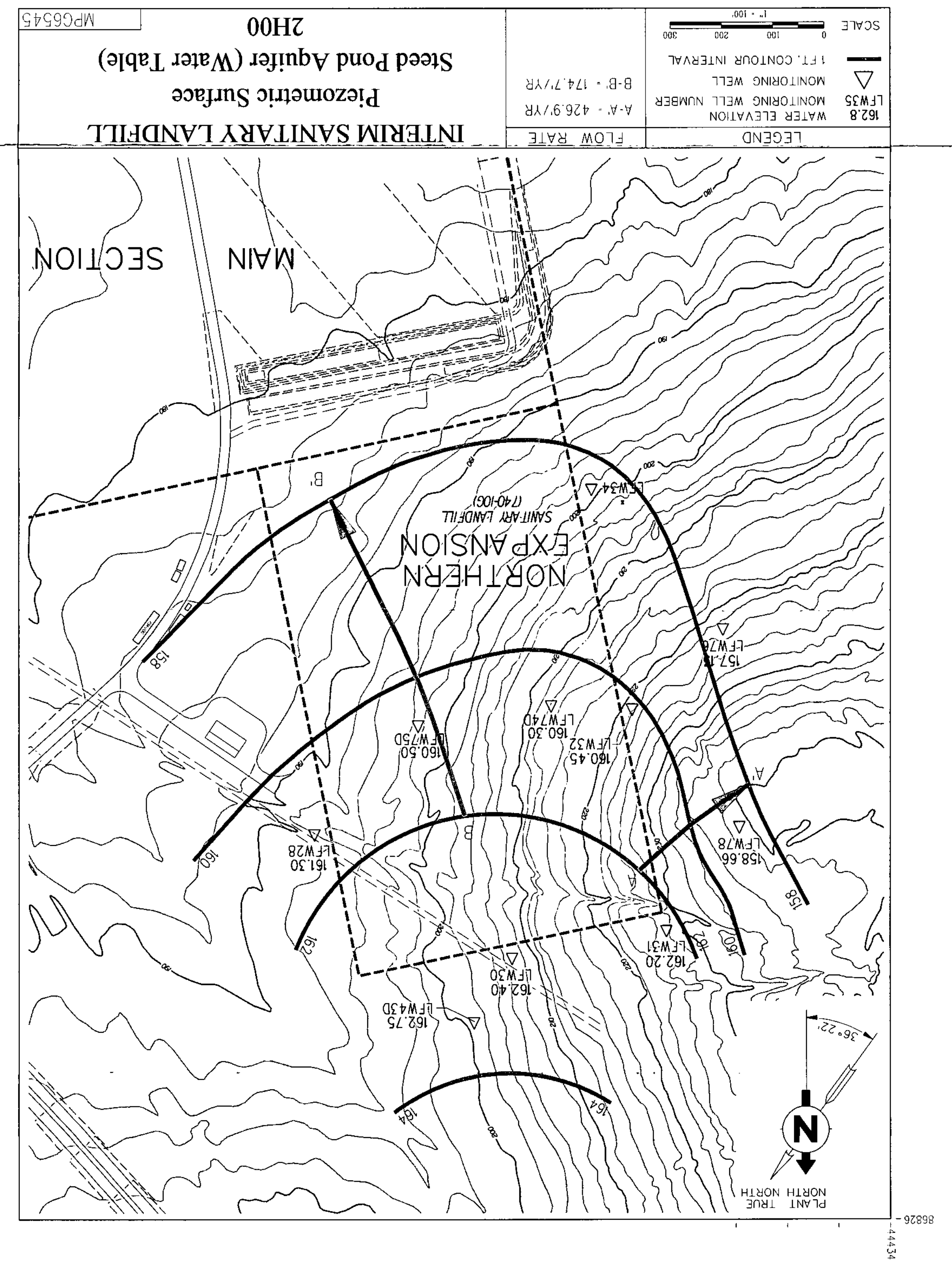




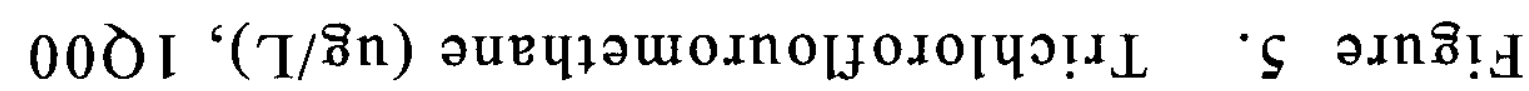

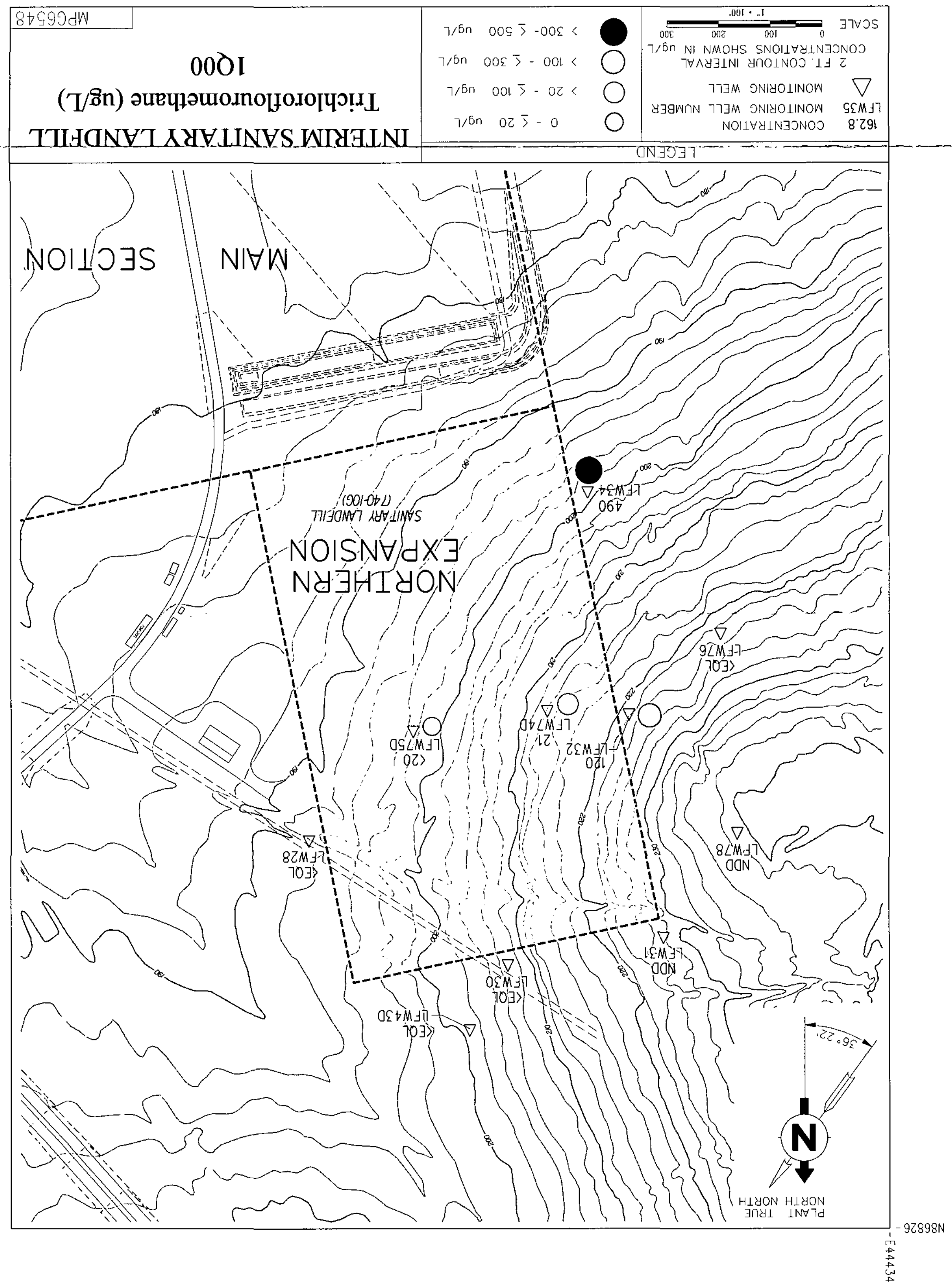

I $00 Z$ XYHกNH :

†9†00-000Z-УL-כySM 


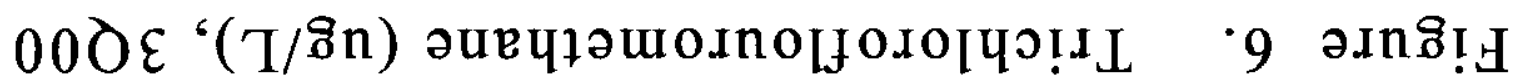

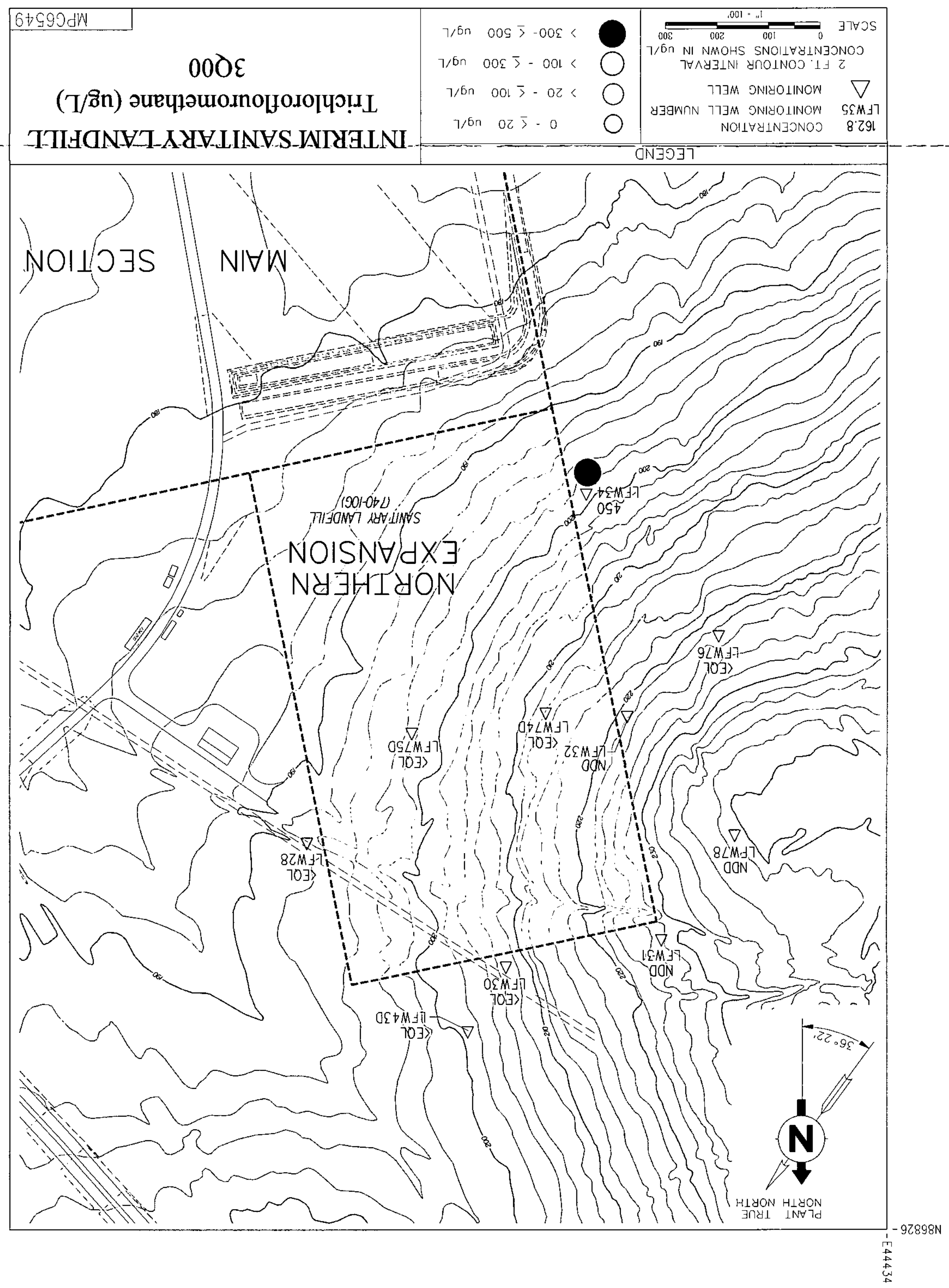

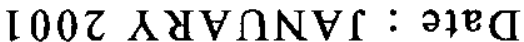

† $9000-000 z-Y . L-J y S M$ 


\section{Appendix D}

\section{Vadose Methane Monitoring Data}


Methane results:

On 10/7/99 4:39 p.m., ADS Laboratory (SRTC/SRS) completed analysis of methane samples submitted the morning of 10/7/99. Analyses were performed by GC-Mass Spec. A $30 \mathrm{~m}$ x $0.53 \mathrm{~mm}$ Carboxen-1004 porous layer open tubular capillary column was used for the separation.

\section{Table D.1 Methane Monitoring Results}

\section{Lab ID\# Station ID Methane, volume\% (1)}

$\begin{array}{llc}\text { 3-134091 } & \text { LGM-1 } & 12.1 \text { vol\% } \\ \text { 3-134092 } & \text { LGM-2 } & 0.51 \text { vol\% } \\ \text { 3-134093 } & \text { LGM-3 } & 3.40 \text { vol } \% \\ \text { 3-134094 } & \text { LGM-4 } & 48.5 \text { vol\% } \\ \text { 3-134094 } & \text { LGM-4 (w/5x dilution) } 53.7 \text { vol\%* }\end{array}$

(1) Results are reported with three significant figures. The last digit is insignificant, but reported

* Includes dilution factor correction to base value on original sample.

Post calibration verification, after the LGM-4 sample was performed to check instrument for calibration drift. The instrument remained in specification for calibration. The $53.7 \mathrm{vol} \%$ value is more reliable since it is within the calibration linear range.

The LEL of methane is $\sim 5 \%$. Hence, any result greater than $5 \%$ is $>$ LEL. 